University of Massachusetts Amherst

ScholarWorks@UMass Amherst

Open Access Dissertations

$9-2013$

\title{
Conditional Gaussian Fluctuations and Refined Asymptotics of the Spin In the Phase-Coexistence Region
}

Jingran Li

University of Massachusetts Amherst

Follow this and additional works at: https://scholarworks.umass.edu/open_access_dissertations

Part of the Mathematics Commons, and the Physics Commons

\section{Recommended Citation}

$\mathrm{Li}$, Jingran, "Conditional Gaussian Fluctuations and Refined Asymptotics of the Spin In the PhaseCoexistence Region" (2013). Open Access Dissertations. 806.

https://doi.org/10.7275/cyyd-zq76 https://scholarworks.umass.edu/open_access_dissertations/806

This Open Access Dissertation is brought to you for free and open access by ScholarWorks@UMass Amherst. It has been accepted for inclusion in Open Access Dissertations by an authorized administrator of ScholarWorks@UMass Amherst. For more information, please contact scholarworks@library.umass.edu. 


\title{
CONDITIONAL GAUSSIAN FLUCTUATIONS AND REFINED ASYMPTOTICS OF THE SPIN IN THE PHASE-COEXISTENCE REGION
}

\author{
A Dissertation Presented \\ by \\ JINGRAN LI
}

Submitted to the Graduate School of the University of Massachusetts Amherst in partial fulfillment of the requirements for the degree of

DOCTOR OF PHILOSOPHY

September 2013

Department of Mathematics and Statistics 
(c) Copyright by JINGRAN LI 2013

All Rights Reserved 


\title{
CONDITIONAL GAUSSIAN FLUCTUATIONS AND REFINED ASYMPTOTICS OF THE SPIN IN THE PHASE-COEXISTENCE REGION
}

\author{
A Dissertation Presented
}

by

JINGRAN LI

Approved as to style and content by:

Richard S. Ellis, Chair

Jonathan Machta, Member

Luc Rey-Bellet, Member

Bruce Turkington, Member

Michael Lavine, Department Head

Department of Mathematics and Statistics 
To my loving parents and husband. 


\section{ACKNOWLEDGMENTS}

The writing of this dissertation has been one of the most significant academic challenges that I have ever had to face. Without the support, patience, and guidance of the following people, this study would not have been completed. It is to them that I owe my deepest gratitude.

Foremost, I would like to express my sincere appreciation to my advisor, Prof. Richard S. Ellis, for his continuous support of my Ph.D study and research, for his patience, caring, motivation, enthusiasm, and immense knowledge. His excellent guidance helped me during all the time that I spent writing this dissertation. His persistent help also inspired me to develop wisdom in dealing with the challenges of life. I can not imagine having a better advisor and mentor for my Ph.D. study.

I am grateful to Prof. Jonathan Machta for several useful discussions about finitesize scaling and for offering me an opportunity to develop my background in computer simulation. I would also like to thank the rest of my dissertation committee, Prof. Luc Rey-Bellet and Prof. Bruce Turkington, for their encouragement, insightful comments, and questions. Finally, I thank Prof. Peter T. Otto for permission to use Figures 1 and 2.

I would like to thank the Department of Mathematics and Statistics at Umass. People here are genuinely nice and I' $\mathrm{m}$ glad to have interacted with many. I am grateful to Prof. Michael Lavine for guidance in statistical projects. I thank Prof. Qianyong Chen and Prof. Hongkun Zhang for their caring and suggestions. Many thanks to Dong Yan (awesome learning partner), Chenyu Wang, Peng Wang, Dechang Chen, Mei Duanmu, Yue Zhao, Kai $\mathrm{Li}$, Weilong $\mathrm{Hu}$, and other classmates for creating an excellent study atmosphere. Konstantinos Gourgoulias shared valuable study information and Tassos Vogiannou provided 
much valued laughter. To my dear Grey's girls, Catherine Benincasa (thanks for making our "chatting" the weekly event to look forward to, for your warm hug and tasted tea), Julie Rana (thanks for your open mind to introduce me into the local culture, for caring about me and being always supportive), Haley Hedlin (thanks for your warm heart and help in statistics), Elizabeth Drellich, and Adena Calden, thank you all for being there to listen, drink, and console.

I also thank my friends (too many to list here but you know who you are!) in the USA, China, and elsewhere for providing support and friendship throughout, some of whom have already been named. Special thanks must go to Dong Yin, Bo Peng, and Qintong He for their genuine companionship and the wonderful time together.

No acknowledgments would be complete without giving thanks to my family. I wish I could show them how much I love and appreciate them as they love me. My parents, Yuru $\mathrm{Li}$ and Lin Chen, are wonderful parents and friends. They've instilled many admirable qualities in me and given me a good foundation with which to meet life. They've taught me about hard work and self-respect, about responsibility and persistence, and about how to be independent. They've given me the belief and strength to chase my dreams, even though we were living in a small under-developed town. My husband, Chaoqun Jia, whose support, encouragement, quiet patience and unwavering love has been sticking by my side during my good and bad times. He has faith in me and allows me to be as ambitious as I wanted. He has been sharing his valuable suggestions with me. He has been non-judgmental of me, appreciating my sense of humor, and inspiring my confidence. 


\title{
ABSTRACT \\ CONDITIONAL GAUSSIAN FLUCTUATIONS AND REFINED ASYMPTOTICS OF THE SPIN IN THE PHASE-COEXISTENCE REGION
}

\author{
SEPTEMBER 2013 \\ JINGRAN LI \\ B.Sc., XI'AN JIAOTONG UNIVERSITY \\ M.Sc., XI'AN JIAOTONG UNIVERSITY \\ Ph.D., UNIVERSITY OF MASSACHUSETTS AMHERST
}

Directed by: Professor Richard S. Ellis

In this dissertation four results are presented on the fluctuations of the spin per site around the thermodynamic magnetization in the mean-field Blume-Capel model, a basic model in statistical mechanics. The first two results refine the main theorem in a 2010 paper by R. S. Ellis, J. Machta, and P. T. Otto published in Annals of Applied Probability 20 (2010) 2118-2161. This paper provides the first rigorous confirmation of the statistical mechanical theory of finite-size scaling for a mean-field model. The first main result studies the asymptotics of the centered, finite-size magnetization, giving its precise rate of convergence to 0 along parameter sequences lying in the phase-coexistence region and converging sufficiently slowly to either a second-order point or the tricritical point of the model. A simple inequality yields our second main result, which generalizes the main theorem in the Ellis-Machta-Otto paper by giving an upper bound on the rate of convergence to 0 of the absolute value of the difference between the finite-size magnetization and the 
thermodynamic magnetization. These first two results have direct relevance to the theory of finite-size scaling. They are consequences of the third main result. This is a new conditional limit theorem for the spin per site, where the conditioning allows us to focus on a neighborhood of the pure states having positive thermodynamic magnetization. The fourth main result is a conditional central limit theorem showing that the fluctuations of the spin per site are Gaussian in a neighborhood of the pure states having positive thermodynamic magnetization. 


\section{TABLE OF CONTENTS}

Page

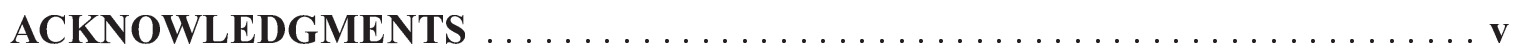

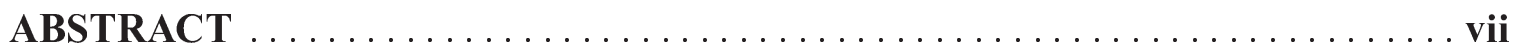

LIST OF TABLES ....................

LIST OF FIGURES $\ldots \ldots \ldots \ldots \ldots \ldots \ldots \ldots \ldots \ldots \ldots \ldots \ldots \ldots \ldots \ldots \ldots \ldots \ldots$

\section{CHAPTER}

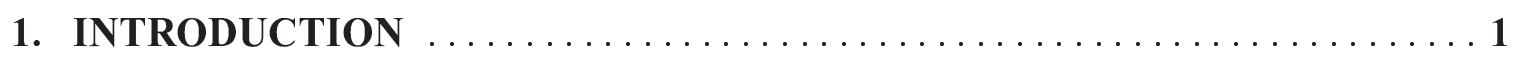

2. PHASE-TRANSITION STRUCTURE OF THE MEAN-FIELD B-C

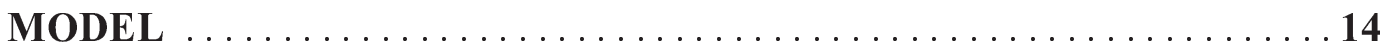

3. ASYMPTOTIC BEHAVIOR OF $E_{n, \beta_{n}, K_{n}}\left\{\left|S_{n} / n\right|\right\} \ldots \ldots \ldots \ldots \ldots \ldots \ldots$

4. ASYMPTOTIC BEHAVIOR OF $E_{n, \beta_{n}, K_{n}}\left\{|| S_{n} / n\left|-m\left(\beta_{n}, K_{n}\right)\right|\right\} \ldots \ldots \ldots 22$

5. VERIFICATION OF HYPOTHESES OF THEOREM 4.1 FOR

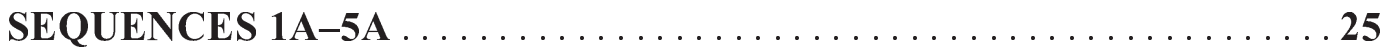

6. PROOF OF PART (A) OF THEOREM $4.1 \ldots \ldots \ldots \ldots \ldots \ldots \ldots \ldots \ldots \ldots$

7. OUTLINE OF THE PROOF OF THEOREM $6.1 \ldots \ldots \ldots \ldots \ldots \ldots \ldots \ldots$

7.1 Outline of the proof of part (b) of Theorem $6.1 \ldots \ldots \ldots \ldots \ldots \ldots \ldots$

7.2 Outline of the proof of part (a) of Theorem $6.1 \ldots \ldots \ldots \ldots \ldots \ldots \ldots \ldots$

8. PREPARATORY LEMMAS FOR PROOF OF PART (B) OF THEOREM

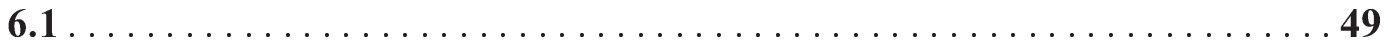

9. PROOF OF PART (B) OF THEOREM $6.1 \ldots \ldots \ldots \ldots \ldots \ldots \ldots \ldots \ldots \ldots$

9.1 Proof of Step 1 in Proof of Theorem $6.1(\mathrm{~b}) \ldots \ldots \ldots \ldots \ldots \ldots \ldots \ldots$ 
9.2 Proof of Substep 2a in Proof of Theorem 6.1 (b) ................ 83

9.3 Proof of Substep $2 b$ in Proof of Theorem 6.1 (b) . . . . . . . . . . . 85

10. PROOF OF PART (A) OF THEOREM $6.1 \ldots \ldots \ldots \ldots \ldots \ldots \ldots \ldots \ldots \ldots \ldots \ldots \ldots$

10.1 Proof of Step 1 in Proof of Theorem 6.1 (a) . . . . . . . . . . . . . 93

10.2 Proof of Substep 2a in Proof of Theorem 6.1 (a) . . . . . . . . . . . . 94

10.3 Proof of Substep $2 b$ in Proof of Theorem 6.1 (a) . . . . . . . . . . . . 97

\section{APPENDICES}

A. PROOF THAT SEQUENCES 1A-5A SATISFY THE LIMITS IN

HYPOTHESIS (III') OF THEOREM $4.1 \ldots \ldots \ldots \ldots \ldots \ldots \ldots \ldots \ldots \ldots$

B. PROOF OF THE MDP IN PART (A) OF THEOREM $6.2 \ldots \ldots \ldots \ldots \ldots \ldots 125$

C. PROOF OF THE REPRESENTATION FORMULA IN LEMMA 8.1 FOR

THE MEAN-FIELD B-C MODEL . . . . . . . . . . . . . . . . . . 134

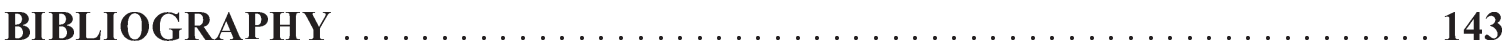




\section{LIST OF TABLES}

Table

Page

1.1 The equations where each of the five sequences is defined and the values

of $\alpha_{0}, \theta$, and $\kappa$ for each sequence. .................... 11

5.1 The equation where each of the 5 sequences is defined and the inequalities on the coefficients guaranteeing that each sequence lies in the phase-coexistence region (Ph-CR) or in the single-phase region (1-PhR). The next-to-last column states the structure of the set $\mathcal{M}_{g}$ of global minimum points of the Ginzburg-Landau polynomial $g$ for each sequence in terms of a positive number $\bar{x}$ that can be explicitly calculated. The theorems in [10] where this information is verified are

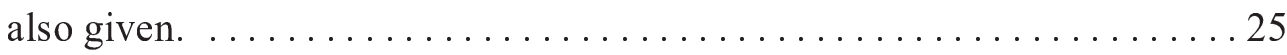




\section{LIST OF FIGURES}

Figure

Page

1 The sets that describe the phase-transition structure of the mean-field B-C

model: the second-order curve $\left\{(\beta, K(\beta)), 0<\beta<\beta_{c}\right\}$, the first-order curve $\left\{\left(\beta, K_{1}(\beta)\right), \beta>\beta_{c}\right\}$, and the tricritical point $\left(\beta_{c}, K\left(\beta_{c}\right)\right)$. The phase-coexistence region consists of all $(\beta, K)$ above the second-order curve, above the tricritical point, on the first-order curve, and above the first-order curve. . . . . . . . . . . 4

2 Possible paths for the five sequences converging to a second-order point and to the tricritical point. In section 5 and appendix A these sequences are defined and are shown to satisfy the hypotheses of Theorem 4.1 and Theorem 6.1. The sequences labeled 1-5 in this figure correspond to sequences $1 \mathrm{a}-5 \mathrm{a}$ in Table 1.1 and Table $5.1 . \ldots \ldots 10$ 


\section{CHAPTER 1 \\ INTRODUCTION}

The purpose of this dissertation is to analyze the asymptotic behavior of the fluctuations of the spin per site around the thermodynamic magnetization along parameter sequences having physical relevance in the mean-field Blume-Capel model. This analysis is presented in three limit theorems and an upper bound. Our research culminates a series of papers that study the phase-transition structure of the model via analytic techniques and probabilistic limit theorems $[14,8,10,13]$. The mean-field Blume-Capel model is a mean-field version of an important lattice model due to Blume and Capel, to which we will refer as the BC model $[3,5,6,7]$. The mean-field B-C model is an important object of study because it is one of the simplest models that exhibits the following complicated phase-transition structure: a curve of second-order points; a curve of first-order points; and a tricritical point, which separates the two curves.

Our first two main results refine the main theorem in [13]. The goal of [13] is to compare the asymptotic behaviors of the thermodynamic magnetization and the finite-size magnetization along parameter sequences lying in the phase coexistence region and converging to either a second-order point or the tricritical point of the mean-field B-C model. Theorem 4.1 in that paper shows that these two quantities are asymptotic when the parameter $\alpha$ controlling the speed at which the sequence approaches criticality is below a certain threshold $\alpha_{0}$. However, when $\alpha$ exceeds $\alpha_{0}$, the thermodynamic magnetization converges to 0 much faster than the finite-size magnetization. These results in [13] are the first rigorous confirmations of the statistical mechanical theory of finite-size scaling for a mean-field model $[1],[13, \S 6]$. 
The importance of both the theory of finite-size scaling and the mean-field B-C model motivate us in this dissertation to refine Theorem 4.1 in [13]. Our first main result studies the asymptotics, along appropriate paramater sequences, of the centered, finite-size magnetization for $0<\alpha<\alpha_{0}$, obtaining its precise rate of convergence to 0 . Our second main result applies a simple inequality that gives an upper bound on the rate of convergence to 0 of the absolute value of the difference between the finite-size magnetization and the thermodynamic magnetization. This upper bound yields the conclusion of Theorem 4.1 in [13] as a corollary and, along with the first main result, has additional relevance to the theory of finite-size scaling discussed below. Our first two main results are stated in (1.4) and (1.6). While Theorem 4.1 in [13] is obtained from a moderate deviation principle, the refinements of that theorem in this dissertation are obtained from our third main result, which is the conditional limit theorem stated in (1.7). Our fourth main result is a conditional central limit theorem stated in (1.9) and showing that the fluctuations of the spin per site are Gaussian in a neighborhood of the pure states having positive thermodynamic magnetization.

The mean-field B-C model is defined by a canonical ensemble that we denote by $P_{N, \beta, K} ; N$ is the number of vertices, $\beta>0$ is the inverse temperature, and $K>0$ is the interaction strength. $P_{N, \beta, K}$ is defined in (2.1) in terms of the Hamiltonian

$$
H_{N, K}(\omega)=\sum_{j=1}^{N} \omega_{j}^{2}-\frac{K}{N}\left(\sum_{j=1}^{N} \omega_{j}\right)^{2}
$$

In this formula $\omega_{j}$ is the spin at site $j \in\{1,2, \ldots, N\}$ and takes values in $\Lambda=\{-1,0,1\}$. The configuration space for the model is the set $\Lambda^{N}$ containing all sequences $\omega=\left(\omega_{1}, \ldots, \omega_{N}\right)$ with each $\omega_{j} \in \Lambda$. Expectation with respect to $P_{N, \beta, K}$ is denoted by $E_{N, \beta, K}$. The finite-size magnetization is defined by $E_{N, \beta, K}\left\{\left|S_{N} / N\right|\right\}$, where $S_{N}$ equals the total spin $\sum_{j=1}^{N} \omega_{j}$.

Before discussing the results in this dissertation, we first summarize the phase-transition structure of the mean-field B-C model as derived in [14]. For $\beta>0$ and $K>0$, we denote by $\mathcal{M}_{\beta, K}$ the set of equilibrium values of the magnetization. The set $\mathcal{M}_{\beta, K}$ coincides with 
the set of global minimum points of the free-energy function $G_{\beta, K}$, which is defined in (2.3)-(2.4). The critical inverse temperature of the mean-field B-C model is $\beta_{c}=\log 4$. For $0<\beta \leq \beta_{c}$ there exists a quantity $K(\beta)$ and for $\beta>\beta_{c}$ there exists a quantity $K_{1}(\beta)$ having the following properties. The positive quantity $m(\beta, K)$ appearing in this list is the thermodynamic magnetization.

1. Fix $0<\beta \leq \beta_{c}$. Then for $0<K \leq K(\beta), \mathcal{M}_{\beta, K}$ consists of the unique pure phase 0 , and for $K>K(\beta), \mathcal{M}_{\beta, K}$ consists of two nonzero values $\pm m(\beta, K)$.

2. For $0<\beta \leq \beta_{c}, \mathcal{M}_{\beta, K}$ undergoes a continuous bifurcation at $K=K(\beta)$, changing continuously from $\{0\}$ for $K \leq K(\beta)$ to $\{ \pm m(\beta, K)\}$ for $K>K(\beta)$. This continuous bifurcation corresponds to a second-order phase transition.

3. Fix $\beta>\beta_{c}$. Then for $0<K<K_{1}(\beta), \mathcal{M}_{\beta, K}$ consists of the unique pure phase 0 ; for $K=K_{1}(\beta), \mathcal{M}_{\beta, K}$ consists of 0 and two nonzero values $\pm m\left(\beta, K_{1}(\beta)\right)$; and for $K>K_{1}(\beta), \mathcal{M}_{\beta, K}$ consists of two nonzero values $\pm m(\beta, K)$.

4. For $\beta>\beta_{c}, \mathcal{M}_{\beta, K}$ undergoes a discontinuous bifurcation at $K=K_{1}(\beta)$, changing discontinuously from $\{0\}$ for $K<K(\beta)$ to $\{0, \pm m(\beta, K)\}$ for $K=K_{1}(\beta)$ to $\{ \pm m(\beta, K)\}$ for $K>K_{1}(\beta)$. This discontinuous bifurcation corresponds to a firstorder phase transition.

Because of item 2, we refer to the curve $\left\{(\beta, K(\beta)), 0<\beta<\beta_{c}\right\}$ as the second-order curve and points on this curve as second-order points. Because of item 4 , we refer to the curve $\left\{\left(\beta, K_{1}(\beta)\right), \beta>\beta_{c}\right\}$ as the first-order curve and points on this curve as first-order points. The point $\left(\beta_{c}, K\left(\beta_{c}\right)\right)=(\log 4,3 / 2 \log 4)$, called the tricritical point, separates the second-order curve from the first-order curve. The phase-coexistence region is defined as the set of all points in the positive $\beta-K$ quadrant for which $\mathcal{M}_{\beta, K}$ consists of more than one value. Therefore the phase-coexistence region consists of all points above the second-order 
curve, above the tricritical point, on the first-order curve, and above the first-order curve; that is,

$$
\left\{(\beta, K): 0<\beta \leq \beta_{c}, K>K(\beta) \text { and } \beta>\beta_{c}, K \geq K_{1}(\beta)\right\}
$$

Figure 1 exhibits the sets that describe the phase-transition structure of mean-field B-C model.

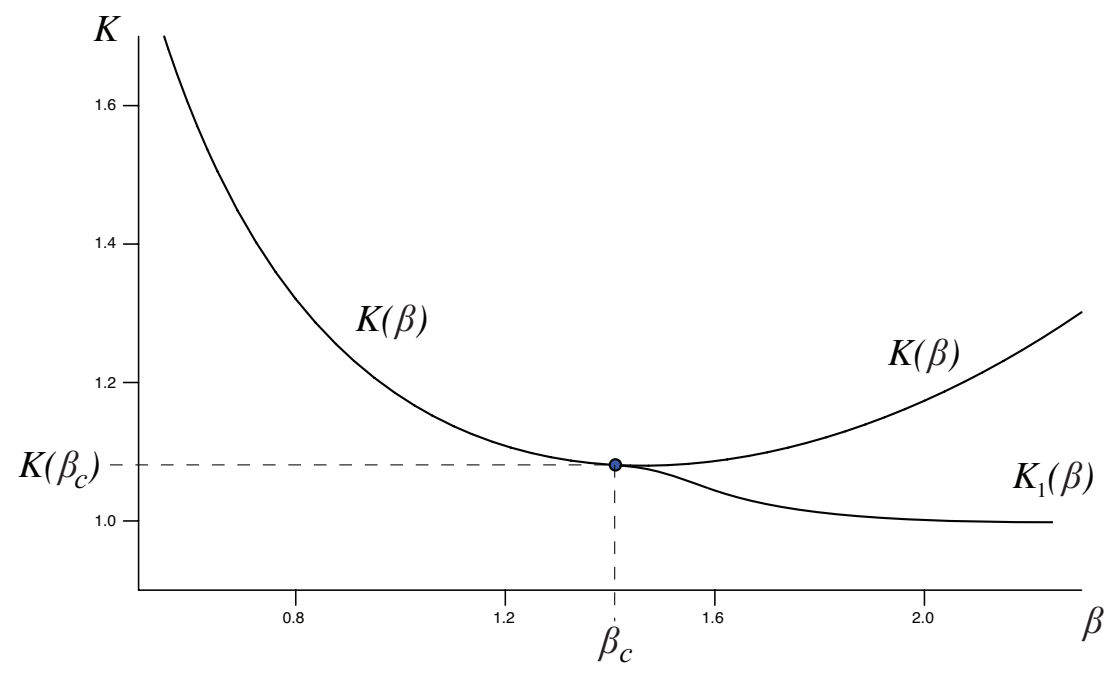

Figure 1. The sets that describe the phase-transition structure of the mean-field B-C model: the second-order curve $\left\{(\beta, K(\beta)), 0<\beta<\beta_{c}\right\}$, the first-order curve $\left\{\left(\beta, K_{1}(\beta)\right), \beta>\right.$ $\left.\beta_{c}\right\}$, and the tricritical point $\left(\beta_{c}, K\left(\beta_{c}\right)\right)$. The phase-coexistence region consists of all $(\beta, K)$ above the second-order curve, above the tricritical point, on the first-order curve, and above the first-order curve.

In order to discuss the contributions of this dissertation, it is helpful first to explain the main results in [10] and [13]. Those papers focus on positive sequences $\left(\beta_{n}, K_{n}\right)$ that lie in the phase-coexistence region for all sufficiently large $n$, converge to either a second-order point or the tricritical point, and satisfy the hypotheses of Theorem 3.2 in [10]. These sequences are parameterized by $\alpha>0$ in the sense that the limits

$$
b=\lim _{n \rightarrow \infty} n^{\alpha}\left(\beta_{n}-\beta\right) \text { and } k=\lim _{n \rightarrow \infty} n^{\alpha}\left(K_{n}-K(\beta)\right)
$$


are assumed to exist and are not both 0 . Six specific such sequences are introduced in section 4 of that paper. Theorem 3.2 in [10] states that for any $\alpha>0, m\left(\beta_{n}, K_{n}\right)$ has the asymptotic behavior

$$
m\left(\beta_{n}, K_{n}\right) \sim \bar{x} / n^{\theta \alpha}
$$

where $\theta>0$ and $\bar{x}$ is the positive global minimum point of a certain polynomial $g(x)$ called the Ginzburg-Landau polynomial. This polynomial is defined in terms of the free-energy function $G_{\beta, K}$ in hypothesis (iii)(a) of Theorem 3.1 below.

One of the surprises in the study of the mean-field B-C model is the appearance of the Ginzburg-Landau polynomial in a number of basic results. These include the asymptotic formula (1.1), the quantity $\bar{z}$ in the asymptotic formula (1.4) and the conditional limit theorem (1.7), the limiting variance in the conditional central limit theorem (1.9), and the rate function in the moderate deviation principle in Theorem 6.2.

A straightforward large-deviation calculation summarized in [13, p. 2120] shows that for fixed $(\beta, K)$ lying in the phase-coexistence region the spin per site $S_{N} / N$ has the weakconverg-ence limit

$$
P_{N, \beta, K}\left\{S_{N} / N \in d x\right\} \Longrightarrow\left(\frac{1}{2} \delta_{m(\beta, K)}+\frac{1}{2} \delta_{-m(\beta, K)}\right)(d x)
$$

This implies that

$$
\lim _{N \rightarrow \infty} E_{N, \beta, K}\left\{\left|S_{N} / N\right|\right\}=m(\beta, K)
$$

Because the thermodynamic magnetization $m(\beta, K)$ is the limit, as the number of spins goes to $\infty$, of the finite-size magnetization $E_{N, \beta, K}\left\{\left|S_{N} / N\right|\right\}$, the thermodynamic magnetization is a physically relevant estimator of the finite-size magnetization, at least when evaluated at fixed $(\beta, K)$ in the phase-coexistence region.

The main focus of [13] is to determine whether the thermodynamic magnetization is a physically relevant estimator of the finite-size magnetization in a more general sense, namely, when evaluated along positive sequences that lie in the phase-coexistence region 
for all sufficiently large $n$, converge to a second-order point or the tricritical point, and satisfy a set of hypotheses including those of Theorem 3.2 in [10]. The criterion for determining whether $m\left(\beta_{n}, K_{n}\right)$ is a physically relevant estimator is that as $n \rightarrow \infty, m\left(\beta_{n}, K_{n}\right)$ is asymptotic to the finite-size magnetization $E_{n, \beta_{n}, K_{n}}\left\{\left|S_{n} / n\right|\right\}$, both of which converge to 0 . In this formulation we let $N=n$ in the finite-size magnetization; i.e., we let the number of spins $N$ coincide with the index $n$ parametrizing the sequence $\left(\beta_{n}, K_{n}\right)$.

As summarized in Theorems 4.1 and 4.2 in [13], the main finding is that $m\left(\beta_{n}, K_{n}\right)$ is a physically relevant estimator when the parameter $\alpha$ controlling the speed at which $\left(\beta_{n}, K_{n}\right)$ approaches criticality is below a certain threshold $\alpha_{0}$. The value of $\alpha_{0}$ depends on the type of the phase transition - first-order, second-order, or tricritical — that influences the sequence, an issue addressed in section 6 of [13]. For $0<\alpha<\alpha_{0}$ this finding is summarized by the limit

$$
\lim _{n \rightarrow \infty} n^{\theta \alpha}\left|E_{n, \beta_{n}, K_{n}}\left\{\left|S_{n} / n\right|\right\}-m\left(\beta_{n}, K_{n}\right)\right|=0
$$

which in combination with (1.1) implies that

$$
E_{n, \beta_{n}, K_{n}}\left\{\left|S_{n} / n\right|\right\} \sim \bar{x} / n^{\theta \alpha} \sim m\left(\beta_{n}, K_{n}\right)
$$

By contrast, when $\alpha>\alpha_{0}, m\left(\beta_{n}, K_{n}\right)$ converges to 0 much faster than $E_{n, \beta_{n}, K_{n}}\left\{\left|S_{n} / n\right|\right\}$. The sequences for which these asymptotic results are valid include the six sequences introduced in $[10, \S 4]$.

We now turn to the main focus of this dissertation, which is a refined analysis of the fluctuations of $S_{n} / n$ around $m\left(\beta_{n}, K_{n}\right)$ for $0<\alpha<\alpha_{0}$. This refined analysis is expressed in three limit theorems and an upper bound. Our first main result involves a quantity that we call the centered, finite-size magnetization. It is defined by $E_{n, \beta_{n}, K_{n}}\left\{|| S_{n} / n \mid-\right.$ $\left.m\left(\beta_{n}, K_{n}\right) \mid\right\}$. Let $\kappa=\frac{1}{2}\left(1-\alpha / \alpha_{0}\right)+\theta \alpha$. For $0<\alpha<\alpha_{0}$ and for a class of sequences that includes the first five sequences introduced in $[10, \S 4]$ part (a) of Theorem 4.1 gives 
the following exact rate of convergence to 0 of the centered, finite-size magnetization as $n \rightarrow \infty:$

$$
E_{n, \beta_{n}, K_{n}}\left\{|| S_{n} / n\left|-m\left(\beta_{n}, K_{n}\right)\right|\right\} \sim \bar{z} / n^{\kappa}
$$

In this formula $\bar{z}=\left(2 /\left[\pi g^{(2)}(\bar{x})\right]\right)^{1 / 2}$, where $g^{(2)}(\bar{x})$ denotes the positive second derivative of the Ginzburg-Landau polynomial $g$ evaluated at its unique positive global minimum point $\bar{x}$. For all $0<\alpha<\alpha_{0}, \kappa$ is larger than $\theta \alpha$. Thus the rate $\bar{z} / n^{\kappa}$ at which $E_{n, \beta_{n}, K_{n}}\left\{|| S_{n} / n\left|-m\left(\beta_{n}, K_{n}\right)\right|\right\}$ converges to 0 is asymptotically faster than the rate $\bar{x} / n^{\theta \alpha}$ at which $E_{n, \beta_{n}, K_{n}}\left\{\left|S_{n} / n\right|\right\}$ and $m\left(\beta_{n}, K_{n}\right)$ converge separately to 0 .

This asymptotic result generalizes (1.3), which is the conclusion of Theorem 4.1 in [13]. To see this, define $A_{n}=E_{n, \beta_{n}, K_{n}}\left\{|| S_{n} / n\left|-m\left(\beta_{n}, K_{n}\right)\right|\right\}$ and note that

$$
\left|E_{n, \beta_{n}, K_{n}}\left\{\left|S_{n} / n\right|\right\}-m\left(\beta_{n}, K_{n}\right)\right| \leq A_{n}
$$

Equation (1.4) states that $\lim _{n \rightarrow \infty} n^{\kappa} A_{n}=\bar{z}$. Since $\kappa>\theta \alpha$, this implies that

$$
0=\lim _{n \rightarrow \infty} n^{\theta \alpha} A_{n} \geq \lim _{n \rightarrow \infty} n^{\theta \alpha}\left|E_{n, \beta_{n}, K_{n}}\left\{\left|S_{n} / n\right|\right\}-m\left(\beta_{n}, K_{n}\right)\right|=0
$$

The fact that this second limit equals 0 yields (1.3), which is the conclusion of Theorem 4.1 in [13].

For any $\varepsilon>0$ and all sufficiently large $n, A_{n} \leq(\bar{z}+\varepsilon) / n^{\kappa}$. Hence (1.5) yields our second main result, which is that for all sufficiently large $n$

$$
\left|E_{n, \beta_{n}, K_{n}}\left\{\left|S_{n} / n\right|\right\}-m\left(\beta_{n}, K_{n}\right)\right| \leq(\bar{z}+\varepsilon) / n^{\kappa}
$$

It is stated in part (b) of Theorem 4.1 .

One of the hypotheses of Theorem 4.1 is that $\theta \alpha_{0}<1 / 2$. Because of this condition, $\kappa=\frac{1}{2}+\alpha\left(\theta-1 /\left(2 \alpha_{0}\right)\right)$ is a decreasing function of $\alpha \in\left(0, \alpha_{0}\right)$, This property yields 
interpretations of (1.4) and (1.6) that are relevant to the theory of finite-size scaling. Because (1.4) is an exact asymptotic result, this property of $\kappa$ shows that the rate at which the centered, finite-size magnetization converges to 0 is slower the closer $\alpha$ is to the threshold value $\alpha_{0}$. We now turn to (1.6). The conclusion of Theorem 4.1 in [13] is that for $0<\alpha<\alpha_{0}$ the finite-size magnetization $E_{n, \beta_{n}, K_{n}}\left\{\left|S_{n} / n\right|\right\}$ and the thermodynamic magnetization are asymptotic to each other. Because (1.6) is an upper bound rather than an exact asymptotic result, the fact that $\kappa$ is a decreasing function of $\alpha \in\left(0, \alpha_{0}\right)$ suggests, but does not prove, that the rate at which $\left|E_{n, \beta_{n}, K_{n}}\left\{\left|S_{n} / n\right|\right\}-m\left(\beta_{n}, K_{n}\right)\right|$ converges to 0 is slower the closer $\alpha$ is to the threshold value $\alpha_{0}$. In the context of the theory of finite-size scaling, these interpretations of (1.4) and (1.6) are certainly intuitive since $\alpha$ controls the speed at which $\left(\beta_{n}, K_{n}\right)$ approaches criticality.

Like the other three main results in this paper, (1.6) is valid only for $0<\alpha<\alpha_{0}$. However, it is worthwhile pointing out that if $\alpha$ equals the threshold value $\alpha_{0}$, then at least in the rate of decay of the right-hand side, (1.6) gives the correct asymptotic rate at which $\left|E_{n, \beta_{n}, K_{n}}\left\{\left|S_{n} / n\right|\right\}-m\left(\beta_{n}, K_{n}\right)\right|$ converges to 0 provided a certain constant is nonzero. In order to see this, we refer to Theorem 4.3 in [13], which shows that for $\alpha=\alpha_{0}$

$$
E_{n, \beta_{n}, K_{n}}\left\{\left|S_{n} / n\right|\right\} \sim \bar{\zeta} / n^{\theta \alpha_{0}}, \text { where } \bar{\zeta}=\frac{1}{\int_{\mathbb{R}} \exp [-g(x)] d x} \cdot \int_{\mathbb{R}}|x| \exp [-g(x)] d x
$$

Since for $\alpha=\alpha_{0}, m\left(\beta_{n}, K_{n}\right) \sim \bar{x} / n^{\theta \alpha_{0}}$ [Thm. 3.1], it follows that when $\bar{\zeta} \neq \bar{x}$,

$$
\left|E_{n, \beta_{n}, K_{n}}\left\{\left|S_{n} / n\right|\right\}-m\left(\beta_{n}, K_{n}\right)\right| \sim|\bar{\zeta}-\bar{x}| / n^{\theta \alpha_{0}}
$$

When $\alpha=\alpha_{0}$, we have $\kappa=\theta \alpha_{0}$. Hence the right-hand side of the last display has the same rate of decay as the right-hand side of (1.6) provided $\bar{\zeta} \neq \bar{x}$. In general we expect that $\bar{\zeta} \neq \bar{x}$. However, when $\bar{\zeta}=\bar{x}$, the right-hand side of the last display equals 0 , and so the last display gives no information. 
The proof of the asymptotic result (1.4) is based on the following new conditional limit stated in part (b) of Theorem 6.1 for $0<\alpha<\alpha_{0}$ :

$$
\lim _{n \rightarrow \infty} n^{\kappa} E_{n, \beta_{n}, K_{n}}\left\{\left|S_{n} / n-m\left(\beta_{n}, K_{n}\right)\right| \mid S_{n} / n>\delta m\left(\beta_{n}, K_{n}\right)\right\}=\bar{z}
$$

The conditioning is on the event $\left\{S_{n} / n>\delta m\left(\beta_{n}, K_{n}\right)\right\}$, where $\delta \in(0,1)$ is sufficiently close to 1 . This conditioning allows us to study the asymptotic behavior of the system in a neighborhood of the pure states having thermodynamic magnetization $m\left(\beta_{n}, K_{n}\right)$. According to Lemma 6.3

$$
\begin{aligned}
\lim _{n \rightarrow \infty} & P_{n, \beta_{n}, K_{n}}\left\{S_{n} / n>\delta m\left(\beta_{n}, K_{n}\right)\right\} \\
\quad= & \lim _{n \rightarrow \infty} P_{n, \beta_{n}, K_{n}}\left\{S_{n} / n<-\delta m\left(\beta_{n}, K_{n}\right)\right\}=1 / 2 .
\end{aligned}
$$

These limits are the analog of the weak convergence limit (1.2), showing that as $n \rightarrow \infty$ the mass of the $P_{n, \beta_{n}, K_{n}}$-distribution of $S_{n} / n$ concentrates at $\pm m\left(\beta_{n}, K_{n}\right)$. As we show in section 6, the limits (1.7) and (1.8) and a moderate deviation estimate on the probability $P_{n, \beta_{n}, K_{n}}\left\{\delta m\left(\beta_{n}\right.\right.$, $\left.\left.K_{n}\right) \geq S_{n} / n \geq-\delta m\left(\beta_{n}, K_{n}\right)\right\}$ yield

$$
\lim _{n \rightarrow \infty} n^{\kappa} E_{n, \beta_{n}, K_{n}}\left\{|| S_{n} / n\left|-m\left(\beta_{n}, K_{n}\right)\right|\right\}=\bar{z}
$$

This limit is equivalent to (1.4). In subsection 7.1 we outline the proof of the conditional limit (1.7), and the details of the proof are given in sections 8 and 9 .

The results (1.4) and (1.6), which are stated in parts (a) and (b) of Theorem 4.1, and the conditional limit (1.7), which is stated in part (b) of Theorem 6.1, are valid for the first five sequences introduced in $[10, \S 4]$. Located in the phase-coexistence region for all sufficiently large $n$, the first two sequences converge to a second-order point, and the last three sequences converge to the tricritical point. Possible paths followed by these sequences 
are shown in Figure 2. For each of the five sequences the quantities $\alpha_{0}, \theta$, and $\kappa$ appearing in Theorem 4.1 are specified in Table 1.1.

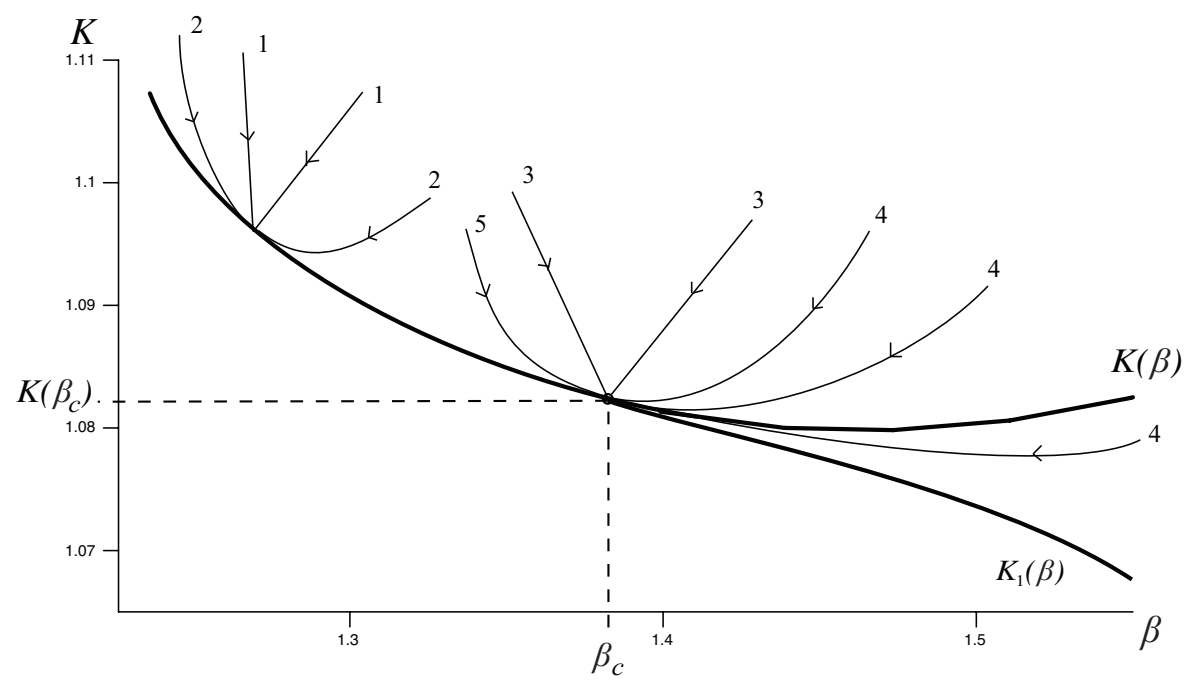

Figure 2. Possible paths for the five sequences converging to a second-order point and to the tricritical point. In section 5 and appendix A these sequences are defined and are shown to satisfy the hypotheses of Theorem 4.1 and Theorem 6.1 . The sequences labeled $1-5$ in this figure correspond to sequences 1a-5a in Table 1.1 and Table 5.1.

The conditional limit (1.7) is closely related to our fourth main result, which is stated in part (a) of Theorem 6.1 and is also valid for the first five sequences introduced in [10, $\S 4]$. This result is a new conditional central limit theorem for the spin for $0<\alpha<\alpha_{0}$. As in (1.7), the conditioning is on the event $\left\{S_{n} / n>\delta m\left(\beta_{n}, K_{n}\right)\right\}$, where $\delta \in(0,1)$ is sufficiently close to 1 . Part (a) of Theorem 6.1 states that when conditioned on $\left\{S_{n} / n>\right.$ $\left.\delta m\left(\beta_{n}, K_{n}\right)\right\}$, the $P_{n, \beta_{n}, K_{n}}$-distributions of $n^{\kappa}\left(S_{n} / n-m\left(\beta_{n}, K_{n}\right)\right)$ converge weakly to a normal random variable $N\left(0,1 / g^{(2)}(\bar{x})\right)$ with mean 0 and variance $1 / g^{(2)}(\bar{x})$; in symbols, 


\begin{tabular}{||l|l|l|l|l||}
\hline \hline Seq. & Defn. & $\boldsymbol{\alpha}_{\mathbf{0}}$ & $\boldsymbol{\theta}$ & $\boldsymbol{\kappa}$ \\
\hline \hline $1 \mathrm{a}$ & $(5.5)$ & $\frac{1}{2}$ & $\frac{1}{2}$ & $\frac{1}{2}(1-\alpha)$ \\
\hline $2 \mathrm{a}$ & $(5.6)$ & $\frac{1}{2 p}$ & $\frac{p}{2}$ & $\frac{1}{2}(1-p \alpha)$ \\
\hline $3 \mathrm{a}$ & $(5.7)$ & $\frac{2}{3}$ & $\frac{1}{4}$ & $\frac{1}{2}(1-\alpha)$ \\
\hline $4 \mathrm{a}$ & $(5.8)$ & $\frac{1}{3}$ & $\frac{1}{2}$ & $\frac{1}{2}(1-2 \alpha)$ \\
\hline $5 \mathrm{a}$ & $(5.10)$ & $\frac{1}{3}$ & $\frac{1}{2}$ & $\frac{1}{2}(1-2 \alpha)$ \\
\hline \hline
\end{tabular}

Table 1.1. The equations where each of the five sequences is defined and the values of $\alpha_{0}$, $\theta$, and $\kappa$ for each sequence.

$$
\begin{gathered}
P_{n, \beta_{n}, K_{n}}\left\{n ^ { \kappa } \left(S_{n} / n-\right.\right. \\
\left.\left.=m\left(\beta_{n}, K_{n}\right)\right) \in d x \mid S_{n} / n>\delta m\left(\beta_{n}, K_{n}\right)\right\} \\
\Longrightarrow N\left(0,1 / g^{(2)}(\bar{x})\right) .
\end{gathered}
$$

Since $\kappa=\frac{1}{2}\left(1-\alpha / \alpha_{0}\right)+\theta \alpha$ is less than $1 / 2$ [Thm. 6.1(c)], the scaling in this result is non-classical. An equivalent formulation is that for any bounded, continuous function $f$

$$
\begin{aligned}
\lim _{n \rightarrow \infty} & E_{n, \beta_{n}, K_{n}}\left\{f\left(n^{\kappa}\left(S_{n} / n-m\left(\beta_{n}, K_{n}\right)\right)\right) \mid S_{n} / n>\delta m\left(\beta_{n}, K_{n}\right)\right\} \\
= & \lim _{n \rightarrow \infty} E_{n, \beta_{n}, K_{n}}\left\{f\left(S_{n} / n^{1-\kappa}-n^{\kappa} m\left(\beta_{n}, K_{n}\right)\right) \mid S_{n} / n>\delta m\left(\beta_{n}, K_{n}\right)\right\} \\
= & E\left\{f\left(N\left(0,1 / g^{(2)}(\bar{x})\right)\right)\right\} \\
= & \frac{1}{\int_{\mathbb{R}} \exp \left[-\frac{1}{2} g^{(2)}(\bar{x}) x^{2}\right] d x} \cdot \int_{\mathbb{R}} f(x) \exp \left[-\frac{1}{2} g^{(2)}(\bar{x}) x^{2}\right] d x .
\end{aligned}
$$

Through the term $g^{(2)}(\bar{x})$ this conditional central limit theorem and the asymptotic formula (1.4) exhibit a sensitive dependence on the choice of the sequence $\left(\beta_{n}, K_{n}\right)$, which lies in the phase coexistence region for all sufficiently large $n$ and converges to a secondorder point or the tricritical point. This contrasts sharply with the central limit theorem that is valid for an arbitrary sequence $\left(\beta_{n}, K_{n}\right)$ that converges to a point $(\beta, K)$ in the singlephase region defined by $\left\{(\beta, K): 0<\beta \leq \beta_{c}, 0<K<K(\beta)\right\}$. In this situation it is proved in Theorem 5.5 in [8] that

$$
P_{n, \beta_{n}, K_{n}}\left\{S_{n} / n^{1 / 2} \in d x\right\} \Longrightarrow N\left(0, \sigma^{2}(\beta, K)\right)
$$


where the limiting variance $\sigma^{2}(\beta, K)$ depends only on $(\beta, K)$ and not on the sequence $\left(\beta_{n}, K_{n}\right)$.

Formally, the conditional limit (1.7) follows from the conditional central limit theorem (1.10) if one replaces the bounded, continuous function $f$ by the absolute value function. Then (1.10) would imply

$$
\begin{aligned}
\lim _{n \rightarrow \infty} & n^{\kappa} E_{n, \beta_{n}, K_{n}}\left\{\left|S_{n} / n-m\left(\beta_{n}, K_{n}\right)\right| \mid S_{n} / n>\delta m\left(\beta_{n}, K_{n}\right)\right\} \\
= & \lim _{n \rightarrow \infty} E_{n, \beta_{n}, K_{n}}\left\{\left|S_{n} / n^{1-\kappa}-n^{\kappa} m\left(\beta_{n}, K_{n}\right)\right| \mid S_{n} / n>\delta m\left(\beta_{n}, K_{n}\right)\right\} \\
= & \frac{1}{\int_{\mathbb{R}} \exp \left[-\frac{1}{2} g^{(2)}(\bar{x}) x^{2}\right] d x} \cdot \int_{\mathbb{R}}|x| \exp \left[-\frac{1}{2} g^{(2)}(\bar{x}) x^{2}\right] d x=\bar{z} .
\end{aligned}
$$

In fact, both the conditional limit (1.7) and the conditional central limit theorem (1.9) are consequences of a weak convergence result stated in (7.10) in this dissertation. The proof of this weak convergence result is outlined in subsection 7.1 and given in Lemma 8.7. The proof of the conditional limit (1.7) is outlined in subsection 7.1 and is given in section 8 and 9. The proof of the conditional central limit theorem (1.9) is outlined in subsection 7.2 and is given in section 8 and 10 .

The contents of this paper are as follows. In section 2 we define the mean-field B-C model and summarize its phase-transition structure in Theorems 2.1 and 2.2. For a class of sequences $\left(\beta_{n}, K_{n}\right)$ lying in the phase-coexistence region for all sufficiently large $n$ and converging either to a second-order point or to the tricritical point, Theorem 3.1 in section 3 describes the asymptotic behavior of $m\left(\beta_{n}, K_{n}\right) \rightarrow 0$ as stated in (1.1). Theorem 3.2 in section 3 states one of the main results of [13], which is that as $n \rightarrow 0, m\left(\beta_{n}, K_{n}\right)$ is asymptotic to $E_{n, \beta_{n}, K_{n}}\left\{\left|S_{n} / n\right|\right\}$ for $0<\alpha<\alpha_{0}$, proving that for this range of $\alpha$ the thermodynamic magnetization $m\left(\beta_{n}, K_{n}\right)$ is a physically relevant estimator of the finitesize magnetization $E_{n, \beta_{n}, K_{n}}\left\{\left|S_{n} / n\right|\right\}$.

The first two main results in this dissertation are given in section 4. According to part (a) of Theorem 4.1, for $0<\alpha<\alpha_{0}$ 


$$
E_{n, \beta_{n}, K_{n}}\left\{\left|S_{n} / n-m\left(\beta_{n}, K_{n}\right)\right|\right\} \sim \bar{z} / n^{\kappa},
$$

where $\bar{z}=\left(2 /\left[\pi g^{(2)}(\bar{x})\right]\right)^{1 / 2}$ and $\kappa=\frac{1}{2}\left(1-\alpha / \alpha_{0}\right)+\theta \alpha$. Part (b) of this theorem gives the inequality (1.6). Parts (a) and (b) of Theorem 4.1 are applied in section 5 to five specific sequences $\left(\beta_{n}, K_{n}\right)$. The first two sequences converge to a second-order point, and the last three sequences converge to the tricritical point. In section 6 part (b) of Theorem 6.1 states the conditional limit (1.7), which is the third main result in this dissertation. From this conditional limit parts (a) and (b) of Theorem 4.1 are derived. Part (a) of Theorem 6.1 states the conditional central limit theorem (1.9), which is the fourth main result in this dissertation. In section 7 we motivate the proofs of parts (a) and (b) of Theorem 6.1. The technical details of the proof of part (b) are given in sections 8 and 9. The analogous but more straightforward proof of part (a) is given in section 10. Several preparatory lemmas in section 8 are also used in section 10 . 


\section{CHAPTER 2}

\section{PHASE-TRANSITION STRUCTURE OF THE MEAN-FIELD B-C MODEL}

For $N \in \mathbb{N}$ the mean-field Blume-Capel model is defined on the complete graph on $N$ vertices $1,2, \ldots, N$. The spin at site $j \in\{1,2, \ldots, N\}$ is denoted by $\omega_{j}$, a quantity taking values in $\Lambda=\{-1,0,1\}$. The Hamiltonian for this model is defined by

$$
H_{N, K}(\omega)=\sum_{j=1}^{N} \omega_{j}^{2}-\frac{K}{N}\left(\sum_{j=1}^{N} \omega_{j}\right)^{2},
$$

where $K>0$ is a positive parameter representing the interaction strength and $\omega=\left(\omega_{1}, \ldots, \omega_{N}\right)$ $\in \Lambda^{N}$. We will refer to this model as the mean-field B-C model.

Let $P_{N}$ be the product measure on $\Lambda^{N}$ with identical one-dimensional marginals $\rho=$ $\frac{1}{3}\left(\delta_{-1}+\delta_{0}+\delta_{1}\right)$. Then $P_{N}$ assigns the probability $3^{-N}$ to each $\omega \in \Lambda^{N}$. For inverse temperature $\beta>0$ and for $K>0$, the canonical ensemble for the mean-field B-C model is the sequence of probability measures that assign to each subset $B$ of $\Lambda^{N}$ the probability

$$
\begin{aligned}
P_{N, \beta, K}(B) & =\frac{1}{Z_{N}(\beta, K)} \cdot \int_{B} \exp \left[-\beta H_{N, K}\right] d P_{N} \\
& =\frac{1}{Z_{N}(\beta, K)} \cdot \sum_{\omega \in B} \exp \left[-\beta H_{N, K}(\omega)\right] \cdot 3^{-N},
\end{aligned}
$$

where

$$
Z_{N}(\beta, K)=\int_{\Lambda^{N}} \exp \left[-\beta H_{N, K}\right] d P_{N}=\sum_{\omega \in \Lambda^{N}} \exp \left[-\beta H_{N, K(\omega)}\right] \cdot 3^{-N}
$$


It is useful to rewrite this measure in a different form. Define $S_{N}(\omega)=\sum_{j=1}^{N} \omega_{j}$ and let $P_{N, \beta}$ be the product measure on $\Lambda_{N}$ with identical one-dimensional marginals

$$
\rho_{\beta}\left(d \omega_{j}\right)=\frac{1}{Z(\beta)} \cdot \exp \left(-\beta \omega_{j}^{2}\right) \rho\left(d \omega_{j}\right)
$$

where $Z(\beta)=\int_{\Lambda} \exp \left(-\beta \omega_{j}^{2}\right) \rho\left(d \omega_{j}\right)=\left(1+2 e^{-\beta}\right) / 3$. Define

$$
P_{N, \beta}(d \omega)=\prod_{j=1}^{N} \rho_{\beta}\left(d \omega_{j}\right)=\frac{1}{[Z(\beta)]^{N}} \prod_{j=1}^{N} \exp \left(-\beta \omega_{j}^{2}\right) \rho\left(d \omega_{j}\right)
$$

and

$$
\tilde{Z}_{N}(\beta, K)=\int_{\Lambda^{N}} \exp \left[N \beta K\left(S_{N} / N\right)^{2}\right] d P_{N, \beta}=\frac{Z_{N}(\beta, K)}{[Z(\beta)]^{N}} .
$$

Then we have

$$
P_{N, \beta, K}(d \omega)=\frac{1}{\tilde{Z}_{N}(\beta, K)} \exp \left[N \beta K\left(S_{N}(\omega) / N\right)^{2}\right] P_{N, \beta}(d \omega) .
$$

For $t \in \mathbb{R}$ and $x \in \mathbb{R}$ we also define the cumulant generating function

$$
c_{\beta}(t)=\log \int_{\Lambda} \exp \left(t \omega_{1}\right) \rho_{\beta}\left(d \omega_{1}\right)=\log \left[\frac{1+e^{-\beta}\left(e^{t}+e^{-t}\right)}{1+2 e^{-\beta}}\right]
$$

and the free-energy function

$$
G_{\beta, K}(x)=\beta K x^{2}-c_{\beta}(2 \beta K x) .
$$

We denote by $\mathcal{M}_{\beta, K}$ the set of equilibrium macrostates of the mean-field B-C model. As shown in Proposition 3.4 in [14], $\mathcal{M}_{\beta, K}$ can be characterized as the set of global minimum points of $G_{\beta, K}$ :

$$
\mathcal{M}_{\beta, K}=\left\{x \in[-1,1]: x \text { is the global minimum points of } G_{\beta, K}(x)\right\}
$$

In [14] $\mathcal{M}_{\beta, K}$ is denoted by $\tilde{\mathcal{E}}_{\beta, K}$. 
The critical inverse temperature of the mean-field $\mathrm{B}-\mathrm{C}$ model is $\beta_{c}=\log 4$. For $0<$ $\beta \leq \beta_{c}$, the next theorem states that $\mathcal{M}_{\beta, K}$ exhibits a continuous bifurcation as $K$ increases through a value $K(\beta)$. This bifurcation corresponds to a second-order phase transition, and the curve $\left\{(\beta, K(\beta)), 0<\beta<\beta_{c}\right\}$ is called the second-order curve. The point $\left(\beta_{c}, K\left(\beta_{c}\right)\right)$ is called the tricritical point. Theorem 2.1 is proved in Theorem 3.6 in [14], where $K(\beta)$ is denoted by $K_{c}^{(2)}(\beta)$.

Theorem 2.1. For $0<\beta \leq \beta_{c}$, we define

$$
K(\beta)=1 /\left[2 \beta c_{\beta}^{\prime \prime}(0)\right]=\left(e^{\beta}+2\right) /(4 \beta) .
$$

For these values of $\beta, \mathcal{M}_{\beta, K}$ has the following structure.

(a) For $0<K \leq K(\beta), \mathcal{M}_{\beta, K}=\{0\}$.

(b) For $K>K(\beta)$, there exists $m(\beta, K)>0$ such that $\mathcal{M}_{\beta, K}=\{ \pm m(\beta, K)\}$.

(c) $m(\beta, K)$ is a positive, increasing, continuous function for $K>K_{c}(\beta)$, and as $K \rightarrow(K(\beta))^{+}, m(\beta, K) \rightarrow 0$. Therefore, $\mathcal{M}_{\beta, K}$ exhibits a continuous bifurcation at $K(\beta)$.

For $\beta>\beta_{c}$, the next theorem states that $\mathcal{M}_{\beta, K}$ exhibits a discontinuous bifurcation as $K$ increases through a value $K_{1}(\beta)$. This bifurcation corresponds to a first-order phase transition, and the curve $\left\{\left(\beta, K_{1}(\beta)\right), \beta>\beta_{c}\right\}$ is called the first-order curve. Theorem 2.2 is proved in Theorem 3.8 in [14].

Theorem 2.2. For $\beta>\beta_{c}, \mathcal{M}_{\beta, K}$ has the following structure in terms of the quantity $K_{1}(\beta)$, denoted by $K_{c}^{(1)}(\beta)$ in [14] and defined implicitly for $\beta>\beta_{c}$ on page 2231 of [14].

(a) For $0<K<K_{1}(\beta), \mathcal{M}_{\beta, K}=\{0\}$.

(b) For $K=K_{1}(\beta)$ there exists $m\left(\beta, K_{1}(\beta)\right)>0$ such that $\mathcal{M}_{\beta, K_{1}(\beta)}=\left\{0, \pm m\left(\beta, K_{1}(\beta)\right)\right\}$.

(c) For $K>K_{1}(\beta)$ there exists $m(\beta, K)>0$ such that $\mathcal{M}_{\beta, K}=\{ \pm m(\beta, K)\}$. 
(d) $m(\beta, K)$ is a positive, increasing, continuous function for $K \geq K_{1}(\beta)$, and as $K \rightarrow K_{1}(\beta)^{+}, m(\beta, K) \rightarrow m\left(\beta, K_{1}(\beta)\right)>0$. Therefore, $\mathcal{M}_{\beta, K}$ exhibits a discontinuous bifurcation at $K_{1}(\beta)$.

The positive quantity $m(\beta, K)$ in Theorems 2.1 and 2.2 is called the thermodynamic magnetization. In the next section we describe the asymptotic behavior of the finite-size magnetization for suitable sequences $\left(\beta_{n}, K_{n}\right)$ and relate this to the asymptotic behavior of the thermodynamic magnetization $m\left(\beta_{n}, K_{n}\right)$. 


\section{CHAPTER 3}

\section{ASYMPTOTIC BEHAVIOR OF $E_{n, \beta_{n}, K_{n}}\left\{\left|S_{n} / n\right|\right\}$}

For $\beta>0$ and $K>0$ the finite-size magnetization is defined as

$$
E_{N, \beta, K}\left\{\left|S_{N} / N\right|\right\}=\int_{\Omega_{N}}\left|S_{N} / N\right| d P_{N, \beta, K}
$$

where $P_{N, \beta, K}$ denotes the measure defined in (2.1)-(2.2). In this section we describe the asymptotic behavior of $E_{n, \beta_{n}, K_{n}}\left\{\left|S_{n} / n\right|\right\}$ for suitable sequences $\left(\beta_{n}, K_{n}\right)$ lying in the phase-coexistence region. In this formulation we let $N=n$ in the finite-size magnetization; i.e., we let the number of spins $N$ coincide with the index $n$ parametrizing the sequence $\left(\beta_{n}, K_{n}\right)$.

The phase-coexistence region is defined as the set of all points in the positive $\beta-K$ quadrant for which $\mathcal{M}_{\beta, K}$ consists of more than one value. According to Theorems 2.1 and 2.2, the phase-coexistence region consists of all points above the second-order curve, above the tricritical point, on the first-order curve, and above the first-order curve; that is,

$$
\left\{(\beta, K): 0<\beta \leq \beta_{c}, K>K(\beta) \text { and } \beta>\beta_{c}, K \geq K_{1}(\beta)\right\}
$$

For a class of sequences $\left(\beta_{n}, K_{n}\right)$ lying in the phase-coexistence region for all sufficiently large $n$ and converging either to a second-order point or to the tricritical point, Theorem 3.1 describes the asymptotic behavior of the thermodynamic magnetization $m\left(\beta_{n}, K_{n}\right) \rightarrow 0$. The asymptotic behavior is related to the unique positive, global minimum point of the Ginzburg-Landau polynomial, which is defined in hypothesis (iii) of the theorem. Theorem 3.1 will be applied a number of times in this dissertation. 
Theorem 3.1 is a special case of the main theorem in [10], Theorem 3.2. In that paper we describe six different sequences that satisfy the hypotheses of Theorem 3.1. The first five of these sequences are revisited in section 5 of this paper, where we show that they satisfy the hypotheses of Theorem 4.1. In that theorem we state our first two main results. These five sequences, labeled 1a-5a, are summarized in Table 5.1.

Theorem 3.1. Let $\left(\beta_{n}, K_{n}\right)$ be a positive sequence that converges either to a second-order point $(\beta, K(\beta)), 0<\beta<\beta_{c}$, or to the tricritical point $\left(\beta_{c}, K\left(\beta_{c}\right)\right)$. We assume that $\left(\beta_{n}, K_{n}\right)$ satisfies the following four hypotheses.

(i) $\left(\beta_{n}, K_{n}\right)$ lies in the phase-coexistence region for all sufficiently large $n$.

(ii) The sequence $\left(\beta_{n}, K_{n}\right)$ is parametrized by $\alpha>0$. This parameter regulates the speed of approach of $\left(\beta_{n}, K_{n}\right)$ to the second-order point or the tricriticalpoint in the following sense:

$$
b=\lim _{n \rightarrow \infty} n^{\alpha}\left(\beta_{n}-\beta\right) \text { and } k=\lim _{n \rightarrow \infty} n^{\alpha}\left(K_{n}-K(\beta)\right)
$$

both exist, and $b$ and $k$ are not both 0 ; if $b \neq 0$, then $b$ equals 1 or -1 .

(iii) There exists an even polynomial g of degree 4 or 6 satisfying $g(x) \rightarrow \infty$ as $|x| \rightarrow \infty$ together with the following two properties; $g$ is called the Ginzburg-Landau polynomial.

(a) There exist $\alpha_{0}>0$ and $\theta>0$ such that for all $\alpha>0$

$$
\lim _{n \rightarrow \infty} n^{\alpha / \alpha_{0}} G_{\beta_{n}, K_{n}}\left(x / n^{\theta \alpha}\right)=g(x)
$$

uniformly for $x$ in compact subsets of $\mathbb{R}$.

(b) There exists $\bar{x}>0$ such that the set of global minimum points of g equals $\{ \pm \bar{x}\}$. 
(iv) Consider $\alpha_{0}>0$ and $\theta>0$ in hypothesis (iii)(a). There exists a polynomial $H$ satisfying $H(x) \rightarrow \infty$ as $|x| \rightarrow \infty$ together with the following property: for all $\alpha \in\left(0, \alpha_{0}\right)$ there exists $R>0$ such that for all $n \in \mathbb{N}$ sufficiently large and for all $x \in \mathbb{R}$ satisfying $\left|x / n^{\theta \alpha}\right|<R, n^{\alpha / \alpha_{0}} G_{\beta_{n}, K_{n}}\left(x / n^{\theta \alpha}\right) \geq H(x)$.

Under hypotheses (i)-(iv), for any $\alpha>0$

$$
m\left(\beta_{n}, K_{n}\right) \sim \bar{x} / n^{\theta \alpha} ; \text { i.e., } \lim _{n \rightarrow \infty} n^{\theta \alpha} m\left(\beta_{n}, K_{n}\right)=\bar{x} \text {. }
$$

If $b \neq 0$, then this becomes $m\left(\beta_{n}, K_{n}\right) \sim \bar{x}\left|\beta-\beta_{n}\right|^{\theta}$.

Theorem 3.2 restates Theorem 4.1 in [13]. The hypotheses are those of Theorem 3.1 for all $0<\alpha<\alpha_{0}$ together with the inequality $0<\theta \alpha_{0}<1 / 2$. These hypotheses are satisfied by sequences $1 \mathrm{a}-5 \mathrm{a}$ in Table 5.1 as well as by a sixth sequence described in Theorem 4.6 in [10]. Part (a) of the next theorem gives the rate at which $E_{n, \beta_{n}, K_{n}}\left\{\left|S_{n} / n\right|\right\} \rightarrow 0$ for $0<$ $\alpha<\alpha_{0}$, and part (b) states that for the same values of $\alpha, E_{n, \beta_{n}, K_{n}}\left\{\left|S_{n} / n\right|\right\} \sim m\left(\beta_{n}, K_{n}\right)$. Thus Theorem 3.2 shows that the asymptotic behavior of $E_{n, \beta_{n}, K_{n}}\left\{\left|S_{n} / n\right|\right\}$ coincides with that of $m\left(\beta_{n}, K_{n}\right)$ for $0<\alpha<\alpha_{0}$. Theorem 4.2 in [13] shows that for $\alpha>\alpha_{0}, m\left(\beta_{n}, K_{n}\right)$ converges to 0 asymptotically faster than $E_{n, \beta_{n}, K_{n}}\left\{\left|S_{n} / n\right|\right\}$.

Theorem 3.2. Let $\left(\beta_{n}, K_{n}\right)$ be a positive sequence parametrized by $\alpha>0$ and converging either to a second-order point $(\beta, K(\beta)), 0<\beta<\beta_{c}$, or to the tricritical point $\left(\beta_{c}, K\left(\beta_{c}\right)\right)$. We assume that $\left(\beta_{n}, K_{n}\right)$ satisfies the hypotheses of Theorem 3.1 for all $0<\alpha<\alpha_{0}$. We also assume the inequality $0<\theta \alpha_{0}<1 / 2$. The following conclusions hold.

(a) For all $0<\alpha<\alpha_{0}$

$$
E_{n, \beta_{n}, K_{n}}\left\{\left|S_{n} / n\right|\right\} \sim \bar{x} / n^{\theta \alpha} ; \text { i.e., } \lim _{n \rightarrow \infty} n^{\theta \alpha} E_{n, \beta_{n}, K_{n}}\left\{\left|S_{n} / n\right|\right\}=\bar{x}
$$

(b) For all $0<\alpha<\alpha_{0}, E_{n, \beta_{n}, K_{n}}\left\{\left|S_{n} / n\right|\right\} \sim m\left(\beta_{n}, K_{n}\right)$. 
In Theorem 4.1 in the next section we state our first two main results on the fluctuations of $S_{n} / n$ around $m\left(\beta_{n}, K_{n}\right)$ for $0<\alpha<\alpha_{0}$. We then explain how Theorem 4.1 generalizes Theorem 3.2. 


\section{CHAPTER 4}

\section{ASYMPTOTIC BEHAVIOR OF $E_{n, \beta_{n}, K_{n}}\left\{|| S_{n} / n\left|-m\left(\beta_{n}, K_{n}\right)\right|\right\}$}

We denote by $E_{n, \beta_{n}, K_{n}}$ expectation with respect to the measure $P_{n, \beta_{n}, K_{n}}$. Theorem 4.1 states two of our main results. In part (a) we investigate the asymptotic behavior of the centered, finite-size magnetization $E_{n, \beta_{n}, K_{n}}\left\{|| S_{n} / n\left|-m\left(\beta_{n}, K_{n}\right)\right|\right\}$ under the hypotheses of Theorem 3.1 and an additional hypothesis (iii'). We show that this quantity is asymptotic to $\bar{z} / n^{\kappa}$, where $\kappa=\frac{1}{2}\left(1-\alpha / \alpha_{0}\right)+\theta \alpha$ and $\bar{z}>0$ is given explicitly. The rate $\bar{z} / n^{\kappa}$ at which $E_{n, \beta_{n}, K_{n}}\left\{|| S_{n} / n\left|-m\left(\beta_{n}, K_{n}\right)\right|\right\}$ converges to 0 is asymptotically faster than the rate $\bar{x} / n^{\theta \alpha}$ at which $E_{n, \beta_{n}, K_{n}}\left\{\mid S_{n} / n\right.$

|\} and $m\left(\beta_{n}, K_{n}\right)$ converge to 0 separately. As shown in the introduction, the inequality in part (b) is a simple consequence of part (a), which in turn yields the conclusion of Theorem 3.2 as a corollary. The relevance of parts (a) and (b) to the theory of finite-size scaling is discussed in the introduction after the inequality (1.6). We comment on the hypotheses of Theorem 4.1 at the end of this section.

Part (a) of Theorem 4.1 is proved in section 6. Part (c) of Theorem 4.1 asserts that the hypotheses of this theorem are satisfied by sequences 1a-5a in Table 5.1. This is discussed in section 5. For each of these sequences the Ginzburg-Landau polynomial has degree 4 or 6.

Theorem 4.1. Let $\left(\beta_{n}, K_{n}\right)$ be a positive sequence converging either to a second-order point $(\beta, K(\beta)), 0<\beta<\beta_{c}$, or to the tricritical point $\left(\beta_{c}, K\left(\beta_{c}\right)\right)$. We assume that $\left(\beta_{n}, K_{n}\right)$ satisfies the hypotheses of Theorem 3.1 for all $0<\alpha<\alpha_{0}$. We also assume the following additional hypothesis on the Ginzburg-Landau polynomial $g$. 
(iii') Assume that $g$ has degree 4 . Then $\theta \alpha_{0}$ lies in the interval $[1 / 4,1 / 2)$. In addition, for all $0<\alpha<\alpha_{0}$ and for $j=2,3,4$

$$
\lim _{n \rightarrow \infty} n^{\alpha / \alpha_{0}-j \theta \alpha} G_{\beta_{n}, K_{n}}^{(j)}\left(m\left(\beta_{n}, K_{n}\right)\right)=g^{(j)}(\bar{x})>0
$$

Assume that $g$ has degree 6 . Then $\theta \alpha_{0}$ lies in the interval $[1 / 6,1 / 2)$. In addition, for all $0<\alpha<\alpha_{0}$ and for $j=2,3,4,5,6$

$$
\lim _{n \rightarrow \infty} n^{\alpha / \alpha_{0}-j \theta \alpha} G_{\beta_{n}, K_{n}}^{(j)}\left(m\left(\beta_{n}, K_{n}\right)\right)=g^{(j)}(\bar{x})>0
$$

For $\alpha \in\left(0, \alpha_{0}\right)$ we also define $\kappa=\frac{1}{2}\left(1-\alpha / \alpha_{0}\right)+\theta \alpha$. Then for all $0<\alpha<\alpha_{0}$ the following conclusions hold.

(a) We have the asymptotic behavior

$$
E_{n, \beta_{n}, K_{n}}\left\{|| S_{n} / n\left|-m\left(\beta_{n}, K_{n}\right)\right|\right\} \sim \bar{z} / n^{\kappa}
$$

where $\bar{z}=\left(2 /\left(\pi g^{(2)}(\bar{x})\right)\right)^{1 / 2}$; i.e., $\lim _{n \rightarrow \infty} n^{\kappa} E_{n, \beta_{n}, K_{n}}\left\{|| S_{n} / n\left|-m\left(\beta_{n}, K_{n}\right)\right|\right\}=\bar{z}$.

(b) For any $\varepsilon>0$ and all sufficiently large $n$

$$
\left|E_{n, \beta_{n}, K_{n}}\left\{\left|S_{n} / n\right|\right\}-m\left(\beta_{n}, K_{n}\right)\right| \leq \frac{\bar{z}+\varepsilon}{n^{\kappa}}
$$

(c) The hypotheses of this theorem are satisfied by sequences 1a-5a in Table 5.1.

The hypotheses of Theorem 4.1 are those of Theorem 3.1 together with the additional hypothesis (iii') for all $0<\alpha<\alpha_{0}$. The latter hypothesis takes two related forms depending on whether $g$ has degree 4 or degree 6 . In this hypothesis, the assumption on $\theta \alpha_{0}$ yields the inequality $0<\theta \alpha_{0}<1 / 2$, which is required by the moderate deviation principle stated in Theorem 6.2. Hypothesis (iii') also assumes both the form of the asymptotic 
behavior of certain derivatives of $n^{\alpha / \alpha_{0}} G_{\beta_{n}, K_{n}}$ evaluated at $m\left(\beta_{n}, K_{n}\right)$ and the positivity of the corresponding derivatives of $g$ evaluated at the positive global minimum point $\bar{x}$. These assumptions are needed in the proof of Lemma 8.5, a key result needed to prove part (b) of Theorem 6.1, which in turn yields part (a) of Theorem 4.1. In particular, the limits in hypothesis (iii') of Theorem 4.1 are required to prove both parts (a) and (b) of Lemma 8.5 while the positivity of $g^{(j)}(\bar{x})$ is needed to prove part (b) of that lemma. The proof of Lemma 8.5 also requires the fact assumed in hypothesis (iii') that $\theta \alpha_{0}$ lies in the interval $[1 / 4,1 / 2)$ or $[1 / 6,1 / 2)$ depending on whether $g$ has degree 4 or degree 6 .

In the next section we outline how to verify the hypotheses of Theorem 4.1 for sequences 1a-5a in Table 5.1. 


\section{CHAPTER 5}

\section{VERIFICATION OF HYPOTHESES OF THEOREM 4.1 FOR SEQUENCES 1A-5A}

Table 5.1 summarizes five sequences $\left(\beta_{n}, K_{n}\right)$ introduced in section 4 of [10]. Depending on the inequalities on the coefficients, sequences $1,2,3$, and 5 each have two cases labeled $\mathrm{a}$ and $\mathrm{b}$, and sequence 4 has three cases labeled $\mathrm{a}, \mathrm{b}$, and $\mathrm{c}$. All five sequences $1 \mathrm{a}-$ 5a lie in the phase-coexistence region for all sufficiently large $n$ as required by hypothesis (i) of Theorem 3.1 .

\begin{tabular}{|c|c|c|c|c|c|c|}
\hline Seq. & Defn. & Case & Ineq. & Region & $\mathcal{M}_{g}$ & Thm. in [10] \\
\hline \multirow[t]{2}{*}{1} & \multirow[t]{2}{*}{$(5.5)$} & $\mathrm{a}$ & $\bar{K}^{\prime}(\beta) b-k<0$ & Ph-CR & \{ $\{ \pm \bar{x}\}$ & \multirow[t]{2}{*}{ Thm.4.1 } \\
\hline & & $\mathrm{b}$ & $K^{\prime}(\beta) b-k>0$ & 1-PhR & $\{0\}$ & \\
\hline \multirow[t]{2}{*}{2} & \multirow[t]{2}{*}{$(5.6)$} & a & $\left(K^{(p)}(\beta)-\ell\right) b^{p}<0$ & Ph-CR & $\{ \pm \bar{x}\}$ & \multirow[t]{2}{*}{ Thm.4.2 } \\
\hline & & $\mathrm{b}$ & $\left(K^{(p)}(\beta)-\ell\right) b^{p}<0$ & 1-PhR & $\{0\}$ & \\
\hline \multirow[t]{2}{*}{3} & \multirow[t]{2}{*}{$(5.7)$} & $\mathrm{a}$ & $K^{\prime}\left(\beta_{c}\right) b-k<0$ & Ph-CR & $\{ \pm \bar{x}\}$ & \multirow[t]{2}{*}{ Thm.4.3 } \\
\hline & & $\mathrm{b}$ & $K^{\prime}\left(\beta_{c}\right) b-k>0$ & 1-PhR & $\{0\}$ & \\
\hline \multirow[t]{3}{*}{4} & \multirow[t]{3}{*}{$(5.8)$} & $\mathrm{a}$ & $\ell>\ell_{c}, \tilde{\ell} \in \mathbb{R}$ & $\mathrm{Ph}-\mathrm{CR}$ & $\{ \pm \bar{x}\}$ & \multirow[t]{3}{*}{ Thm.4.4 } \\
\hline & & $\mathrm{b}$ & $\ell=\ell_{c}, \ell>K_{1}^{\prime \prime \prime}\left(\beta_{c}\right)$ & Ph-CR & $\{0, \pm \bar{x}\}$ & \\
\hline & & $\mathrm{c}$ & $\ell<\ell_{c}, \ell \in \mathbb{R}$ & 1-PhR & $\{0\}$ & \\
\hline \multirow[t]{2}{*}{5} & \multirow[t]{2}{*}{$(5.10)$} & $\mathrm{a}$ & $\bar{\ell}>K^{\prime \prime}\left(\beta_{c}\right)$ & Ph-CR & $\{ \pm \bar{x}\}$ & \multirow[t]{2}{*}{ Thm.4.5 } \\
\hline & & $\mathrm{b}$ & $\ell<K^{\prime \prime}\left(\beta_{c}\right)$ & 1-PhR & $\{0\}$ & \\
\hline
\end{tabular}

Table 5.1. The equation where each of the 5 sequences is defined and the inequalities on the coefficients guaranteeing that each sequence lies in the phase-coexistence region (Ph-CR) or in the single-phase region (1-PhR). The next-to-last column states the structure of the set $\mathcal{M}_{g}$ of global minimum points of the Ginzburg-Landau polynomial $g$ for each sequence in terms of a positive number $\bar{x}$ that can be explicitly calculated. The theorems in [10] where this information is verified are also given.

The hypotheses of Theorem 4.1 consist of the hypotheses of Theorem 3.1 for all $0<$ $\alpha<\alpha_{0}$ and hypothesis (iii'). Hypothesis (iii') takes two forms depending on the degree 
of the Ginzburg-Landau polynomial $g$. When $g$ has degree $4, \theta \alpha_{0}$ is assumed to lie in the interval $[1 / 4,1 / 2)$ and for all $\alpha \in\left(0, \alpha_{0}\right)$ and for $j=2,3,4$

$$
\lim _{n \rightarrow \infty} n^{\alpha / \alpha_{0}-j \theta \alpha} G_{\beta_{n}, K_{n}}^{(j)}\left(m\left(\beta_{n}, K_{n}\right)\right)=g^{(j)}(\bar{x})>0
$$

When $g$ has degree $6, \theta \alpha_{0}$ is assumed to lie in the interval $[1 / 6,1 / 2)$ and for all $\alpha \in\left(0, \alpha_{0}\right)$ and for $j=2,3,4,5,6$

$$
\lim _{n \rightarrow \infty} n^{\alpha / \alpha_{0}-j \theta \alpha} G_{\beta_{n}, K_{n}}^{(j)}\left(m\left(\beta_{n}, K_{n}\right)\right)=g^{(j)}(\bar{x})>0 .
$$

In this section we verify for sequences $1 \mathrm{a}-5 \mathrm{a}$ that when $g$ has degree 4 , we have $\theta \alpha_{0} \in$ $[1 / 4,1 / 2)$ and $g^{(j)}(\bar{x})>0$ for $j=2,3,4$ and that when $g$ has degree 6 , we have $\theta \alpha_{0} \in$ $[1 / 6,1 / 2)$ and $g^{(j)}(\bar{x})>0$ for $j=2,3,4,5,6$. The verification of the limits in (5.1) and (5.2) is carried out in appendix A.

Sequence 6 introduced in Theorem 4.6 in [10] does not satisfy hypothesis (iii') in Theorem 4.1. In this case $g$ has degree 4 , but $\theta \alpha_{0}$ does not lie in the interval $[1 / 4,1 / 2)$.

The first two sequences converge to a second-order point $(\beta, K(\beta)), 0<\beta<\beta_{c}$, and the last three sequences converge to the tricritical point $\left(\beta_{c}, K\left(\beta_{c}\right)\right)$. For each sequence 1a-5a, the hypotheses of Theorem 3.1 are verified in Theorems 4.1-4.5 in [10]. We follow the same method used in that paper to verify hypothesis (iii') in Theorem 4.1 for sequences 1a-5a. Hypothesis (iii') of Theorem 4.1 takes two forms depending on whether the degree of the Ginzburg-Landau polynomial $g$ is 4 or 6 . We must verify that $\theta \alpha_{0}$ lies in a certain interval and that

$$
\lim _{n \rightarrow \infty} n^{\alpha / \alpha_{0}-j \theta \alpha} G_{\beta_{n}, K_{n}}^{(j)}\left(m\left(\beta_{n}, K_{n}\right)\right)=g^{(j)}(\bar{x})>0
$$

for $j=2,3,4$ when $g$ has degree 4 and for $j=2,3,4,5,6$ when $g$ has degree 6 . The function $G_{\beta, K}$ is defined in $(2.3)-(2.4)$. 
It is straightforward to show that the limit in (5.3) holds for a given $j$ provided the following limit holds uniformly for $x$ in compact subsets of $\mathbb{R}$ :

$$
\lim _{n \rightarrow \infty} n^{\alpha / \alpha_{0}-j \theta \alpha} G_{\beta_{n}, K_{n}}^{(j)}\left(x / n^{\theta \alpha}\right)=g^{(j)}(x)
$$

As shown in Lemma A.1, the proof that the uniform convergence in (5.4) implies the limit in (5.3) uses the fact that $n^{\theta \alpha} m\left(\beta_{n}, k_{n}\right) \rightarrow \bar{x}$ [Thm. 3.1]. The uniform convergence in (5.4) can be obtained formally by taking the $j$-th derivative of the uniform convergence limits in hypothesis (iii)(a) of Theorem 3.1:

$$
\lim _{n \rightarrow \infty} n^{\alpha / \alpha_{0}} G_{\beta_{n}, K_{n}}\left(x / n^{\theta \alpha}\right)=g(x) .
$$

The verification of the uniform convergence limits in (5.4), and thus the verification of the limits (5.1) and (5.2) in hypothesis (iii'), depend on asymptotic properties of the Taylor expansions of $G_{\beta_{n}, K_{n}}^{(j)}\left(x / n^{\theta \alpha}\right)$. This analysis closely parallels the proof of Theorem 3.1, which is based on a similar analysis of the Taylor expansions of $G_{\beta_{n}, K_{n}}\left(x / n^{\theta \alpha}\right)$ carried out in [10]. The details of the calculations can be found in appendix A.

We now define the five sequences $\left(\beta_{n}, K_{n}\right)$ and summarize the verification of the hypotheses of Theorem 4.1 for them.

\section{Sequence 1a}

Definition. Given $0<\beta<\beta_{c}, \alpha>0, b \in\{1,0,-1\}$, and $k \in \mathbb{R}, k \neq 0$, sequence 1 is defined by

$$
\beta_{n}=\beta+b / n^{\alpha} \text { and } K_{n}=K(\beta)+k / n^{\alpha} .
$$

This sequence converges to the second-order point $(\beta, K(\beta))$ along a ray with slope $k / b$ if $b \neq 0$. We assume that $K^{\prime}(\beta) b-k<0$. Under this assumption it is proved in Theorem 4.1 in [10] that sequence 1 satisfies the hypotheses of Theorem 3.1 with $\alpha_{0}=1 / 2$ and $\theta=1 / 2$. When $K^{\prime}(\beta) b-k<0$, we refer to sequence 1 as sequence 1 a. 
Hypothesis (iii') in Theorem 4.1 for sequence 1a. Since $\alpha_{0}=1 / 2$ and $\theta=1 / 2, \theta \alpha_{0}$ lies in the interval $[1 / 4,1 / 2)$ as required by hypothesis (iii'). The limits in (5.1) for $j=2,3,4$ are proved in appendix A. We now prove that $g^{(j)}(\bar{x})>0$ for $j=2,3,4$ using the formulas for $g$ and $\bar{x}$ in Theorem 4.1 in [10]. Let $c_{4}(\beta)=\left(e^{\beta}+2\right)^{2}\left(4-e^{\beta}\right) / 8 \cdot 4$ !. Since $0<\beta<$ $\beta_{c}=\log 4$, we have $e^{\beta}<e^{\beta_{c}}=4$, which implies $c_{4}(\beta)>0$. Since $K^{\prime}(\beta) b-k<0$, these formulas yield

$$
\begin{gathered}
g^{(2)}(\bar{x})=2 \beta\left(K^{\prime}(\beta) b-k\right)+3 \cdot 4 c_{4}(\beta) \bar{x}^{2}=4 \beta\left(k-K^{\prime}(\beta) b\right)>0 \\
g^{(3)}(\bar{x})=4 ! c_{4}(\beta) \bar{x}>0, \text { and } g^{(4)}(\bar{x})=4 ! c_{4}(\beta)>0 .
\end{gathered}
$$

Thus under the condition $K^{\prime}(\beta) b-k<0$ sequence 1a satisfies all the hypotheses of Theorem 4.1.

\section{Sequence 2a}

Definition. Given $0<\beta<\beta_{c}, \alpha>0, b \in\{1,-1\}$, an integer $p \geq 2$, and a real number $\ell \neq K^{(p)}(\beta)$, sequence 2 is defined by

$$
\beta_{n}=\beta+b / n^{\alpha} \text { and } K_{n}=K(\beta)+\sum_{j=1}^{p-1} K^{(j)}(\beta) b^{j} /\left(j ! n^{j \alpha}\right)+\ell b^{p} /\left(p ! n^{p \alpha}\right) .
$$

This sequence converges to the second-order point $(\beta, K(\beta))$ along a curve that coincides with the second-order curve to order $n^{-(p-1) \alpha}$. We assume that $\left(K^{(p)}(\beta)-\ell\right) b^{p}<0$. Under this assumption it is proved in Theorem 4.2 in [10] that sequence 2 satisfies the hypotheses of Theorem 3.1 with $\alpha_{0}=1 /(2 p)$ and $\theta=p / 2$. When $\left(K^{(p)}(\beta)-\ell\right) b^{p}<0$, we refer to sequence 2 as sequence $2 \mathrm{a}$.

Hypothesis (iii') in Theorem 4.1 for sequence 2a. Since $\alpha_{0}=1 /(2 p)$ and $\theta=p / 2, \theta \alpha_{0}$ lies in the interval $[1 / 4,1 / 2)$ as required by hypothesis (iii'). The limits in (5.1) for $j=2,3,4$ 
are proved in appendix A. We now prove that $g^{(j)}(\bar{x})>0$ for $j=2,3,4$ using the formulas for $g$ and $\bar{x}$ in Theorem 4.2 in [10]. Let $c_{4}(\beta)=\left(e^{\beta}+2\right)^{2}\left(4-e^{\beta}\right) / 8 \cdot 4$ !. Since $0<\beta<$ $\beta_{c}=\log 4$, we have $e^{\beta}<e^{\beta_{c}}=4$, which implies $c_{4}(\beta)>0$. Since $\left(K^{(p)}(\beta)-\ell\right) b^{p}<0$, these formulas yield

$$
\begin{gathered}
g^{(2)}(\bar{x})=\frac{2}{p !} \beta\left(K^{(p)}(\beta)-\ell\right) b^{p}+3 \cdot 4 c_{4}(\beta) \bar{x}^{2}=\frac{4}{p !} \beta\left(\ell-K^{(p)}(\beta)\right) b^{p}>0, \\
g^{(3)}(\bar{x})=4 ! c_{4}(\beta) \bar{x}>0, \text { and } g^{(4)}(\bar{x})=4 ! c_{4}(\beta)>0 .
\end{gathered}
$$

Thus under the condition $\left(K^{(p)}(\beta)-\ell\right) b^{p}<0$ sequence 2 a satisfies all the hypotheses of Theorem 4.1.

\section{Sequence 3a}

Definition. Given $\alpha>0, b \in\{1,0,-1\}$, and $k \in \mathbb{R}, k \neq 0$, sequence 3 is defined by

$$
\beta_{n}=\beta_{c}+b / n^{\alpha} \text { and } K_{n}=K\left(\beta_{c}\right)+k / n^{\alpha}
$$

This sequence converges to the tricritical point $\left(\beta_{c}, K\left(\beta_{c}\right)\right)$ along a ray with slope $k / b$ if $b \neq 0$. We assume that $K^{\prime}\left(\beta_{c}\right) b-k<0$. Under this assumption it is proved in Theorem 4.3 in [10] that sequence 3 satisfies the hypotheses of Theorem 3.1 with $\alpha_{0}=2 / 3$ and $\theta=1 / 4$. When $K^{\prime}\left(\beta_{c}\right) b-k<0$, we refer to sequence 3 as sequence 3 a.

Hypothesis (iii') in Theorem 4.1 for sequence 3a. Since $\alpha_{0}=2 / 3$ and $\theta=1 / 4, \theta \alpha_{0}$ lies in the interval $[1 / 6,1 / 2)$ as required by hypothesis (iii'). The limits in (5.2) for $j=2,3,4,5,6$ are proved in appendix A. We now prove that $g^{(j)}(\bar{x})>0$ for $j=2,3,4,5,6$ using the formulas for $g$ and $\bar{x}$ in Theorem 4.3 in [10]. Let $c_{6}=9 / 40$. Since $K^{\prime}\left(\beta_{c}\right) b-k<0$, these formulas yield

$$
g^{(2)}(\bar{x})=2 \beta_{c}\left(K^{\prime}\left(\beta_{c}\right) b-k\right)+5 \cdot 6 c_{6} \bar{x}^{4}=8 \beta_{c}\left(k-K^{\prime}\left(\beta_{c}\right) b\right)>0,
$$




$$
\begin{gathered}
g^{(3)}(\bar{x})=4 \cdot 5 \cdot 6 c_{6} \bar{x}^{3}>0, g^{(4)}(\bar{x})=3 \cdot 4 \cdot 5 \cdot 6 c_{6} \bar{x}^{2}>0 \\
g^{(5)}(\bar{x})=6 ! c_{6} \bar{x}>0, \text { and } g^{(6)}(\bar{x})=6 ! c_{6}>0
\end{gathered}
$$

Thus under the condition $K^{\prime}\left(\beta_{c}\right) b-k<0$ sequence 3 a satisfies all the hypotheses of Theorem 4.1.

\section{Sequence 4a}

Definition. Given $\alpha>0$, a curvature parameter $\ell \in \mathbb{R}$, and another parameter $\tilde{\ell} \in \mathbb{R}$, sequence 4 is defined by

$$
\beta_{n}=\beta_{c}+1 / n^{\alpha} \text { and } K_{n}=K\left(\beta_{c}\right)+K^{\prime}\left(\beta_{c}\right) / n^{\alpha}+\ell /\left(2 n^{\alpha}\right)+\tilde{\ell} /\left(6 n^{3 \alpha}\right) .
$$

This sequence converges from the right to the tricritical point $\left(\beta_{c}, K\left(\beta_{c}\right)\right)$ along the curve $(\beta, \tilde{K}(\beta))$, where for $\beta>\beta_{c}$

$$
\tilde{K}(\beta)=K\left(\beta_{c}\right)+K^{\prime}\left(\beta_{c}\right)\left(\beta-\beta_{c}\right)+\ell\left(\beta-\beta_{c}\right)^{2} / 2+\tilde{\ell}\left(\beta-\beta_{c}\right)^{3} / 6
$$

The first-order curve $\left\{\left(\beta, K_{1}(\beta)\right), \beta>\beta_{c}\right\}$ is shown in Figure 1 in the introduction. In order to determine a condition on the coefficients guaranteeing that sequence 4 satisfies the hypotheses of Theorem 3.1, we must study $K_{1}(\beta)$ more closely.

Since $\lim _{\beta \rightarrow \beta_{c}^{+}} K_{1}(\beta)=K\left(\beta_{c}\right)$ [14, Sects. 3.1, 3.3], by continuity we extend the definition of $K_{1}(\beta)$ from $\beta>\beta_{c}$ to $\beta=\beta_{c}$ by define $K_{1}\left(\beta_{c}\right)=K\left(\beta_{c}\right)$. In addition we must assume other properties of $K_{1}$ that are stated in conjectures 1 and 2 on page 119 of [10]. As a preliminary to stating these conjectures, we assume that the first three right-hand derivatives of $K_{1}(\beta)$ exist at $\beta_{c}$ and denote them by $K_{1}^{\prime}\left(\beta_{c}\right), K_{1}^{\prime \prime}\left(\beta_{c}\right)$, and $K_{1}^{\prime \prime \prime}\left(\beta_{c}\right)$. We also define $\ell_{c}=K^{\prime \prime}\left(\beta_{c}\right)-5 /\left(4 \beta_{c}\right)$. Conjectures 1 and 2 state the following: (1) $K_{1}^{\prime}\left(\beta_{c}\right)=$ $K^{\prime}\left(\beta_{c}\right)$ and (2) $K_{1}^{\prime \prime}\left(\beta_{c}\right)=\ell_{c}<0<K^{\prime \prime}\left(\beta_{c}\right)$. These conjectures are discussed in detail in section 5 of [11] and are supported by properties of the Ginzburg-Landau polynomials and numerical calculations. 
We assume that $\ell>\ell_{c}$, which by conjecture 1 equals $K_{1}^{\prime \prime}\left(\beta_{c}\right)$. Under this assumption it is proved in Theorem 4.4 in [10] that sequence 4 satisfies the hypotheses of Theorem 3.1 with $\alpha_{0}=1 / 3$ and $\theta=1 / 2$. When $\ell>\ell_{c}$, we refer to sequence 4 as sequence 4 a.

Hypothesis (iii') in Theorem 4.1 for sequence 4a. Since $\alpha_{0}=1 / 3$ and $\theta=1 / 2, \theta \alpha_{0}$ lies in the interval $[1 / 6,1 / 2)$ as required by hypothesis (iii'). The limits in (5.2) for $j=2,3,4,5,6$ are proved in appendix A. Define

$$
y=\left(1+\frac{3}{5} \beta_{c}\left(\ell-K^{\prime \prime}\left(\beta_{c}\right)\right)\right)^{1 / 2}
$$

Since $\ell>\ell_{c}=K^{\prime \prime}\left(\beta_{c}\right)-5 /\left(4 \beta_{c}\right)$, we have $y>1 / 2$. We now prove that $g^{(j)}(\bar{x})>0$ for $j=2,3,4,5,6$ using the formulas for $g$ and $\bar{x}$ in Theorem 4.4 in [10]. Let $c_{4}=3 / 16$ and $c_{6}=9 / 40$. These formulas yield

$$
\begin{gathered}
g^{(2)}(\bar{x})=\beta_{c}\left(K^{\prime \prime}\left(\beta_{c}\right)-\ell\right)-3 \cdot 4 \cdot 4 c_{4} \bar{x}^{2}+5 \cdot 6 c_{6} \bar{x}^{4}=\frac{20}{3} y^{2}+\frac{20}{3} y>0 \\
g^{(3)}(\bar{x})=-4 ! \cdot 4 c_{4} \bar{x}+4 \cdot 5 \cdot 6 c_{6} \bar{x}^{3}=9 \bar{x}\left(\frac{4}{3}+\frac{10}{3} y\right)>0 \\
g^{(4)}(\bar{x})=-4 ! \cdot 4 c_{4}+3 \cdot 4 \cdot 5 \cdot 6 c_{6} \bar{x}^{2}=-18+90(1+y)>0 \\
g^{(5)}(\bar{x})=6 ! c_{6} \bar{x}>0, \text { and } g^{(6)}(\bar{x})=6 ! c_{6}>0 .
\end{gathered}
$$

Thus under the condition $\ell>\ell_{c}=K_{1}^{\prime \prime}\left(\beta_{c}\right)$ sequence 4 a satisfies all the hypotheses of Theorem 4.1.

\section{Sequence 5a}


Definition. Given $\alpha>0$ and a real number $\ell \neq K^{\prime \prime}\left(\beta_{c}\right)$, sequence 5 is defined by

$$
\beta_{n}=\beta_{c}-1 / n^{\alpha} \text { and } K_{n}=K\left(\beta_{c}\right)-K^{\prime}\left(\beta_{c}\right) / n^{\alpha}+\ell /\left(2 n^{\alpha}\right) .
$$

This sequence converges to the tricritical point $\left(\beta_{c}, K\left(\beta_{c}\right)\right)$ from the left along the curve that coincide with the second-order curve to order 2 in powers of $\beta-\beta_{c}$. We assume that $\ell>K^{\prime \prime}\left(\beta_{c}\right)$. Under this assumption it is proved in Theorem 4.5 in [10] that sequence 5 satisfies the hypotheses of Theorem 3.1 with $\alpha_{0}=1 / 3$ and $\theta=1 / 2$. When $\ell>K^{\prime \prime}\left(\beta_{c}\right)$, we refer to sequence 5 as sequence $5 \mathrm{a}$.

Hypothesis (iii') in Theorem 4.1 for sequence $5 \mathrm{a}$. Since $\alpha_{0}=1 / 3$ and $\theta=1 / 2, \theta \alpha_{0}$ lies in the interval $[1 / 6,1 / 2)$ as required by hypothesis (iii'). The limits in (5.2) for $j=2,3,4,5,6$ are proved in appendix A. Define $y$ as in (5.9). Since $\ell>K^{\prime \prime}\left(\beta_{c}\right)$, we have $y>1$. We now prove that $g^{(j)}(\bar{x})>0$ for $j=2,3,4,5,6$ using the formulas for $g$ and $\bar{x}$ in Theorem 4.5 in [10]. Let $c_{4}=3 / 16$ and $c_{6}=9 / 40$. These formulas yield

$$
\begin{gathered}
g^{(2)}(\bar{x})=\beta_{c}\left(K^{\prime \prime}\left(\beta_{c}\right)-\ell\right)+3 \cdot 4 \cdot 4 c_{4} \bar{x}^{2}+5 \cdot 6 c_{6} \bar{x}^{4}=\frac{20}{3} y(y-1)>0, \\
g^{(3)}(\bar{x})=4 ! \cdot 4 c_{4} \bar{x}+4 \cdot 5 \cdot 6 c_{6} \bar{x}^{3}>0, g^{(4)}(\bar{x})=4 ! \cdot 4 c_{4}+3 \cdot 4 \cdot 5 \cdot 6 c_{6} \cdot \bar{x}^{2}>0, \\
g^{(5)}(\bar{x})=6 ! c_{6} \bar{x}>0, \text { and } g^{(6)}(\bar{x})=6 ! c_{6}>0 .
\end{gathered}
$$

Thus under the condition $\ell>K^{\prime \prime}\left(\beta_{c}\right)$ sequence 5a satisfies all the hypotheses of Theorem 4.1.

We have completed the discussion of the verification of the hypotheses of Theorem 4.1 for sequences 1a-5a in Table 5.1. This is the content of part (c) of Theorem 4.1. Part (a) of that theorem is proved in the next section. 


\section{CHAPTER 6}

\section{PROOF OF PART (A) OF THEOREM 4.1}

Theorem 6.1, a new theorem stated in this section, has two parts. Under the same hypotheses as Theorem 4.1, part (a) of Theorem 6.1 states a conditional central limit theorem: conditioned on the event $\left\{S_{n} / n>\delta m\left(\beta_{n}, K_{n}\right)\right\}$ for $\delta \in(0,1)$ sufficiently close to 1 , the $P_{n, \beta_{n}, K_{n}}$-distributions of $n^{\kappa}\left(S_{n} / n-m\left(\beta_{n}, K_{n}\right)\right)$ converge weakly to an $N\left(0,1 / g^{(2)}(\bar{x})\right)$ random variable with mean 0 and variance $1 / g^{(2)}(\bar{x})$. Under the same hypotheses as Theorem 4.1, part (b) of Theorem 6.1 states the related conditional limit

$$
\begin{gathered}
\lim _{n \rightarrow \infty} n^{\kappa} E_{n, \beta_{n}, K_{n}}\left\{\left|S_{n} / n-m\left(\beta_{n}, K_{n}\right)\right| \mid S_{n} / n>\delta m\left(\beta_{n}, K_{n}\right)\right\} \\
=E\left\{\left|N\left(0,1 / g^{(2)}(\bar{x})\right)\right|\right\}=\left(2 /\left(\pi g^{(2)}(\bar{x})\right)\right)^{1 / 2}=\bar{z} .
\end{gathered}
$$

We now comment on the proof of part (a) of Theorem 4.1 from part (b) of Theorem 6.1. In Lemma 6.3, we show that the moderate deviation principle in Theorem 6.2 and the asymptotic behavior of $m\left(\beta_{n}, K_{n}\right)$ in Theorem 3.1 imply that the event $\left\{S_{n} / n>\right.$ $\left.\delta m\left(\beta_{n}, K_{n}\right)\right\}$ and the symmetric event $\left\{S_{n} / n<-\delta m\left(\beta_{n}, K_{n}\right)\right\}$ have large probability and that the event $\left\{\delta m\left(\beta_{n}, K_{n}\right)>S_{n} / n>-\delta m\left(\beta_{n}, K_{n}\right)\right\}$ has an exponentially small probability. As we show at the end of this section, combining part (b) of Theorem 6.1 with Lemma 6.3 and using symmetry yield

$$
\lim _{n \rightarrow \infty} n^{\kappa} E_{n, \beta_{n}, K_{n}}\left\{|| S_{n} / n\left|-m\left(\beta_{n}, K_{n}\right)\right|\right\}=\bar{z}
$$

This is part (a) of Theorem 4.1 . 
The proofs of parts (a) and (b) of Theorem 6.1 are long and technical. In section 7 we outline first the proof of part (b) of Theorem 6.1 and then the proof of part (a). As we will see, the latter follows the pattern of proof of part (b) but is more straightforward. Part (b) of Theorem 6.1 is proved in subsections 9.1, 9.2, and 9.3, using a number of preparatory lemmas in section 8. Part (a) of Theorem 6.1 is proved in subsections 10.1, 10.2, and 10.3, using several preparatory lemmas in section 8 . The weak convergence result proved in Lemma 8.7 is the seed that yields both the conditional central limit theorem in part (a) of Theorem 6.1 and the conditional limit in part (b) of Theorem 6.1.

The hypotheses of Theorem 6.1 coincide with the hypotheses of Theorem 4.1. Part (c) of Theorem 6.1 states that for $\alpha \in\left(0, \alpha_{0}\right), \kappa=\frac{1}{2}\left(1-\alpha / \alpha_{0}\right)+\theta \alpha$ lies in the interval $\left(\theta \alpha_{0}, 1 / 2\right)$. This fact is needed in the proofs of Lemmas 8.2, 8.5, 9.4, and 10.4. The proof that $\kappa \in\left(\theta \alpha_{0}, 1 / 2\right)$ is elementary. By hypothesis (iii') of Theorem 4.1, we have $\theta \alpha_{0}<1 / 2$, which gives $\theta<1 /\left(2 \alpha_{0}\right)$. Therefore

$$
\kappa=\frac{1}{2}\left(1-\alpha / \alpha_{0}\right)+\theta \alpha=\frac{1}{2}+\alpha\left(\theta-1 /\left(2 \alpha_{0}\right)\right)<1 / 2
$$

Since $0<\alpha<\alpha_{0}$ and $\theta<1 /\left(2 \alpha_{0}\right)$, we have $\kappa>\frac{1}{2}+\alpha_{0}\left(\theta-1 /\left(2 \alpha_{0}\right)\right)=\theta \alpha_{0}$. This completes the proof of part (c) of Theorem 6.1.

Concerning part (d) of Theorem 6.1, the hypotheses of this theorem coincide with the hypotheses of Theorem 4.1. Thus, as shown in section 5 of this paper and in appendix A, these hypotheses are satisfied by sequences 1a-5a in Table 5.1 .

Theorem 6.1. Let $\left(\beta_{n}, K_{n}\right)$ be a positive sequence converging either to a second-order point $(\beta, K(\beta)), 0<\beta<\beta_{c}$, or to the tricritical point $\left(\beta_{c}, K\left(\beta_{c}\right)\right)$. We assume that for all $0<\alpha<\alpha_{0},\left(\beta_{n}, K_{n}\right)$ satisfies the hypotheses of Theorem 4.1, which coincide with the hypotheses of Theorem 3.1 together with hypothesis (iii'). For $\alpha \in\left(0, \alpha_{0}\right)$ we define $\kappa=\frac{1}{2}\left(1-\alpha / \alpha_{0}\right)+\theta \alpha$. Then for any $0<\alpha<\alpha_{0}$ there exists $\Delta \in(0,1)$ such that for any $\delta \in(\Delta, 1)$ the following conclusions hold. 
(a) When conditioned on the event $\left\{S_{n} / n>\delta m\left(\beta_{n}, K_{n}\right)\right\}$, the $P_{n, \beta_{n}, K_{n}}$-distributions of $n^{\kappa}\left(S_{n} / n-m\left(\beta_{n}, K_{n}\right)\right)$ converge weakly to a normal random variable $N\left(0,1 / g^{(2)}(\bar{x})\right)$ with mean 0 and variance $1 / g^{(2)}(\bar{x})$; in symbols,

$$
P_{n, \beta_{n}, K_{n}}\left\{n^{\kappa}\left(S_{n} / n-m\left(\beta_{n}, K_{n}\right)\right) \in d x \mid S_{n} / n>\delta m\left(\beta_{n}, K_{n}\right)\right\} \Longrightarrow N\left(0,1 / g^{(2)}(\bar{x})\right) .
$$

(b) We have the conditional limit

$$
\begin{aligned}
\lim _{n \rightarrow \infty} & n^{\kappa} E_{n, \beta_{n}, K_{n}}\left\{\left|S_{n} / n-m\left(\beta_{n}, K_{n}\right)\right| \mid S_{n} / n>\delta m\left(\beta_{n}, K_{n}\right)\right\} \\
\quad= & \lim _{n \rightarrow \infty} E_{n, \beta_{n}, K_{n}}\left\{\left|S_{n} / n^{1-\kappa}-n^{\kappa} m\left(\beta_{n}, K_{n}\right)\right| \mid S_{n} / n>\delta m\left(\beta_{n}, K_{n}\right)\right\}=\bar{z},
\end{aligned}
$$

where

$$
\begin{aligned}
\bar{z} & =E\left\{\left|N\left(0,1 / g^{(2)}(\bar{x})\right)\right|\right\} \\
& =\frac{1}{\int_{\mathbb{R}} \exp \left[-\frac{1}{2} g^{(2)}(\bar{x}) x^{2}\right] d x} \cdot \int_{\mathbb{R}}|x| \exp \left[-\frac{1}{2} g^{(2)}(\bar{x}) x^{2}\right] d x=\left(\frac{2}{\pi g^{(2)}(\bar{x})}\right)^{1 / 2}
\end{aligned}
$$

(c) For $\alpha \in\left(0, \alpha_{0}\right), \kappa=\frac{1}{2}\left(1-\alpha / \alpha_{0}\right)+\theta \alpha$ lies in the interval $\left(\theta \alpha_{0}, 1 / 2\right)$.

(d) The hypotheses of this theorem are satisfied by sequences 1a-5a in Table 5.1.

In part (a) of Theorem 6.2 we state a moderate deviation principle (MDP) for the meanfield B-C model. This MDP will be used to prove Lemma 6.3, which in turn will be used to prove part (a) of Theorem 4.1 from part (b) of Theorem 6.1. The rate function in the MDP is the continuous function $\Gamma(x)=g(x)-\inf _{y \in \mathbb{R}} g(y)$, where $g$ is the associated Ginzburg-Landau polynomial. $\Gamma$ satisfies $\Gamma(x) \rightarrow \infty$ as $|x| \rightarrow \infty$. For $A$ a subset of $\mathbb{R}$ define $\Gamma(A)=\inf _{x \in A} \Gamma(x)$.

Theorem 6.2. Let $\left(\beta_{n}, K_{n}\right)$ be a positive sequence converging either to a second-order point $(\beta, K(\beta)), 0<\beta<\beta_{c}$, or to the tricritical point $\left(\beta_{c}, K\left(\beta_{c}\right)\right)$. We assume that 
$\left(\beta_{n}, K_{n}\right)$ satisfies the hypotheses of Theorem 4.1 for all $0<\alpha<\alpha_{0}$. The following conclusions hold.

(a) For all $0<\alpha<\alpha_{0}, S_{n} / n^{1-\theta \alpha}$ satisfies the MDP with respect to $P_{n, \beta_{n}, K_{n}}$ with exponential speed $n^{1-\alpha / \alpha_{0}}$ and rate function $\Gamma(x)=g(x)-\inf _{y \in \mathbb{R}} g(y)$; i.e., for any closed set $F$ in $\mathbb{R}$

$$
\limsup _{n \rightarrow \infty} \frac{1}{n^{1-\alpha / \alpha_{0}}} \log P_{n, \beta_{n}, K_{n}}\left\{S_{n} / n^{1-\theta \alpha} \in F\right\} \leq-\Gamma(F)
$$

and for any open set $G$ in $\mathbb{R}$

$$
\liminf _{n \rightarrow \infty} \frac{1}{n^{1-\alpha / \alpha_{0}}} \log P_{n, \beta_{n}, K_{n}}\left\{S_{n} / n^{1-\theta \alpha} \in G\right\} \geq-\Gamma(G)
$$

(b) The hypotheses of this theorem are satisfied by sequences 1a-5a in Table 5.1 .

The MDP in part (a) of Theorem 6.2 is proved like the MDP in part (a) of Theorem 8.1 in [8] with only changes in notation. Because of the importance of part (a) of Theorem 6.2 , it is proved in appendix B. Concerning part (b) of Theorem 6.2, the hypotheses of this theorem coincide with the hypotheses of Theorem 4.1. Thus, as shown in section 5 of this paper and in appendix A, these hypotheses are satisfied by sequences 1a-5a in Table 5.1.

After proving the next lemma, we use it to derive part (a) of Theorem 4.1 from part (b) of Theorem 6.1.

Lemma 6.3. We assume that $\left(\beta_{n}, K_{n}\right)$ satisfies the hypotheses of Theorem 4.1 for all $0<$ $\alpha<\alpha_{0}$. Then for any $0<\alpha<\alpha_{0}$ and any $\delta \in(0,1)$ there exists $c>0$ such that for all sufficiently large $n$

$$
P_{n, \beta_{n}, K_{n}}\left\{\delta m\left(\beta_{n}, K_{n}\right) \geq S_{n} / n \geq-\delta m\left(\beta_{n}, K_{n}\right)\right\} \leq \exp \left[-c n^{1-\alpha / \alpha_{0}}\right] \rightarrow 0 \text { as } n \rightarrow \infty
$$

In addition,

$$
\lim _{n \rightarrow \infty} P_{n, \beta_{n}, K_{n}}\left\{S_{n} / n>\delta m\left(\beta_{n}, K_{n}\right)\right\}=\lim _{n \rightarrow \infty} P_{n, \beta_{n}, K_{n}}\left\{S_{n} / n<-\delta m\left(\beta_{n}, K_{n}\right)\right\}=1 / 2 .
$$


Proof. By hypothesis (iii)(b) of Theorem 3.1 the global minimum points of $g$ are $\pm \bar{x}$, and by Theorem 3.1, $n^{\theta \alpha} m\left(\beta_{n}, K_{n}\right) \rightarrow \bar{x}$ as $n \rightarrow \infty$. Thus we can choose $\varepsilon>0$ satisfying $(1+\varepsilon) \delta<1$ such that $n^{\theta \alpha} m\left(\beta_{n}, K_{n}\right) \leq(1+\varepsilon) \bar{x}$ for large $n$. Let $F$ be the closed set $[-(1+\varepsilon) \delta \bar{x},(1+\varepsilon) \delta \bar{x}]$. Since $(1+\varepsilon) \delta \bar{x}<\bar{x}$ and $-(1+\varepsilon) \delta \bar{x}>-\bar{x}$, we have

$$
\inf _{y \in F} g(y)>\inf _{z \in \mathbb{R}} g(z)=g(\bar{x})
$$

which implies

$$
\Gamma(F)=\inf _{y \in F}\left\{g(y)-\inf _{z \in \mathbb{R}} g(z)\right\}>0 .
$$

We write $m_{n}=m\left(\beta_{n}, K_{n}\right)$. The moderate deviation upper bound in part (a) of Theorem 6.2 implies that for all sufficiently large $n$

$$
\begin{aligned}
& P_{n, \beta_{n}, K_{n}}\left\{\delta m_{n} \geq S_{n} / n \geq-\delta m_{n}\right\} \\
& \quad=P_{n, \beta_{n}, K_{n}}\left\{\delta n^{\theta \alpha} m_{n} \geq S_{n} / n^{1-\theta \alpha} \geq-\delta n^{\theta \alpha} m_{n}\right\} \\
& \quad \leq P_{n, \beta_{n}, K_{n}}\left\{(1+\varepsilon) \delta \bar{x} \geq S_{n} / n^{1-\theta \alpha} \geq-(1+\varepsilon) \delta \bar{x}\right\} \\
& \quad \leq \exp \left[-n^{1-\alpha / \alpha_{0}} \Gamma(F) / 2\right] \rightarrow 0 \text { as } n \rightarrow \infty .
\end{aligned}
$$

This yields the first assertion in the lemma.

To prove the second assertion, we write

$$
\begin{aligned}
1=P_{n, \beta_{n}, K_{n}}\left\{S_{n} / n \in \mathbb{R}\right\}= & P_{n, \beta_{n}, K_{n}}\left\{\delta m_{n} \geq S_{n} / n \geq-\delta m_{n}\right\} \\
& +P_{n, \beta_{n}, K_{n}}\left\{S_{n} / n>\delta m_{n}\right\} \\
& +P_{n, \beta_{n}, K_{n}}\left\{S_{n} / n<-\delta m_{n}\right\},
\end{aligned}
$$

Symmetry and the first assertion imply that

$$
\lim _{n \rightarrow \infty} P_{n, \beta_{n}, K_{n}}\left\{S_{n} / n>\delta m_{n}\right\}=\lim _{n \rightarrow \infty} P_{n, \beta_{n}, K_{n}}\left\{S_{n} / n<-\delta m_{n}\right\}=1 / 2 .
$$

This completes the proof of the lemma. 
Now we are ready to prove part (a) of Theorem 4.1 .

Proof of part (a) of Theorem 4.1 from part (b) of Theorem 6.1 and Lemma 6.3. We write $m_{n}=m\left(\beta_{n}, K_{n}\right)$. Define

$$
\begin{gathered}
p_{n, \delta}^{+}=P_{n, \beta_{n}, K_{n}}\left\{S_{n} / n>\delta m_{n}\right\}, p_{n, \delta}^{-}=P_{n, \beta_{n}, K_{n}}\left\{S_{n} / n<-\delta m_{n}\right\}, \\
q_{n, \delta}=P_{n, \beta_{n}, K_{n}}\left\{\delta m_{n} \geq S_{n} / n \geq-\delta m_{n}\right\} .
\end{gathered}
$$

Since by symmetry $p_{n, \delta}^{+}=p_{n, \delta}^{-}$and

$$
\begin{aligned}
& E_{n, \beta_{n}, K_{n}}\left\{|| S_{n} / n\left|-m_{n}\right| \mid S_{n} / n<-\delta m_{n}\right\} \\
& =E_{n, \beta_{n}, K_{n}}\left\{|| S_{n} / n\left|-m_{n}\right| \mid S_{n} / n>\delta m_{n}\right\} \\
& =E_{n, \beta_{n}, K_{n}}\left\{\left|S_{n} / n-m_{n}\right| \mid S_{n} / n>\delta m_{n}\right\},
\end{aligned}
$$

we have

$$
\begin{aligned}
E_{n, \beta_{n}, K_{n}}\left\{|| S_{n} / n\left|-m_{n}\right|\right\}= & E_{n, \beta_{n}, K_{n}}\left\{|| S_{n} / n\left|-m_{n}\right| \mid S_{n} / n>\delta m_{n}\right\} \cdot p_{n, \delta}^{+} \\
& +E_{n, \beta_{n}, K_{n}}\left\{|| S_{n} / n\left|-m_{n}\right| \mid S_{n} / n<-\delta m_{n}\right\} \cdot p_{n, \delta}^{-} \\
& +E_{n, \beta_{n}, K_{n}}\left\{|| S_{n} / n\left|-m_{n}\right| \mid \delta m_{n} \geq S_{n} / n \geq-\delta m_{n}\right\} \cdot q_{n, \delta} \\
= & 2 \cdot E_{n, \beta_{n}, K_{n}}\left\{\left|S_{n} / n-m_{n}\right| \mid S_{n} / n>\delta m_{n}\right\} \cdot p_{n, \delta}^{+} \\
& +E_{n, \beta_{n}, K_{n}}\left\{|| S_{n} / n\left|-m_{n}\right| \mid \delta m_{n} \geq S_{n} / n \geq-\delta m_{n}\right\} \cdot q_{n, \delta} .
\end{aligned}
$$

By part (b) of Theorem 6.1 and Lemma 6.3

$$
\lim _{n \rightarrow \infty} n^{\kappa} E_{n, \beta_{n}, K_{n}}\left\{\left|S_{n} / n-m_{n}\right| \mid S_{n} / n>\delta m_{n}\right\} \cdot p_{n, \delta}^{+}=\frac{1}{2} \bar{z}
$$


Since $\left|S_{n} / n\right| \leq 1$ and $0 \leq m_{n} \leq 1$, Lemma 6.3 implies that there exists $c>0$ such that for all sufficiently large $n$

$$
\begin{gathered}
n^{\kappa} E_{n, \beta_{n}, K_{n}}\left\{|| S_{n} / n\left|-m_{n}\right| \mid \delta m_{n} \geq S_{n} / n \geq-\delta m_{n}\right\} \cdot q_{n, \delta} \\
\leq 2 n^{\kappa} q_{n, \delta} \leq 2 n^{\kappa} \exp \left[-c n^{1-\alpha / \alpha_{0}}\right] \rightarrow 0 \text { as } n \rightarrow \infty .
\end{gathered}
$$

It follows that

$$
\lim _{n \rightarrow \infty} n^{\kappa} E_{n, \beta_{n}, K_{n}}\left\{|| S_{n} / n\left|-m_{n}\right|\right\}=\frac{1}{2} \bar{z}+\frac{1}{2} \bar{z}=\bar{z} .
$$

Part (a) of Theorem 4.1 is proved.

In the next section we outline the proof of Theorem 6.1 . 


\section{CHAPTER 7}

\section{OUTLINE OF THE PROOF OF THEOREM 6.1}

In this section we motivate the proof of parts (a) and (b) of Theorem 6.1. We first focus on the proof of part (b) in subsection 7.1 because it is somewhat more complicated than the proof of part (a). The numerous and subtle technical details of the proof of part (b) of Theorem 6.1 are given in sections 8 and 9. The proof of part (a) is outlined in subsection 7.2, and the details of the proof are given in section 10 .

Let $\left(\beta_{n}, K_{n}\right)$ be a positive sequence. Throughout this section we work with $0<\alpha<\alpha_{0}$ and denote $m\left(\beta_{n}, K_{n}\right)$ by $m_{n}$. Let $W_{n}$ be a sequence of normal random variables with mean 0 and variance $\left(2 \beta_{n} K_{n}\right)^{-1}$ defined on a probability space $(\Omega, \mathcal{F}, Q)$. We denote by $\tilde{E}_{n, \beta_{n}, K_{n}}$ expectation with respect to the product measure $P_{n, \beta_{n}, K_{n}} \times Q ; P_{n, \beta_{n}, K_{n}}$ is defined in $(2.1)-(2.2)$.

\subsection{Outline of the proof of part (b) of Theorem 6.1}

We define $\kappa=\frac{1}{2}\left(1-\alpha / \alpha_{0}\right)+\theta \alpha$. Part (b) of Theorem 6.1 states that there exists $\Delta \in(0,1)$ such that for any $\delta \in(\Delta, 1)$

$$
\begin{aligned}
\lim _{n \rightarrow \infty} & n^{\kappa} E_{n, \beta_{n}, K_{n}}\left\{\left|S_{n} / n-m_{n}\right| \mid S_{n} / n>\delta m_{n}\right\} \\
= & \lim _{n \rightarrow \infty} E_{n, \beta_{n}, K_{n}}\left\{\left|S_{n} / n^{1-\kappa}-n^{\kappa} m_{n}\right| \mid S_{n} / n>\delta m_{n}\right\} \\
= & \frac{1}{\int_{\mathbb{R}} \exp \left[-\frac{1}{2} g^{(2)}(\bar{x}) x^{2}\right] d x} \cdot \int_{\mathbb{R}}|x| \exp \left[-\frac{1}{2} g^{(2)}(\bar{x}) x^{2}\right] d x=\bar{z} .
\end{aligned}
$$


The key idea in proving (7.1) is to show that adding suitably scaled versions of the normal random variables $W_{n}$ yields a quantity with the following two properties: its limit equals the last line of (7.1) and the second line of (7.1) has the same limit; specifically,

$$
\begin{aligned}
\lim _{n \rightarrow \infty} & E_{n, \beta_{n}, K_{n}}\left\{\left|S_{n} / n^{1-\kappa}-n^{\kappa} m_{n}\right| \mid S_{n} / n>\delta m_{n}\right\} \\
& =\lim _{n \rightarrow \infty} \tilde{E}_{n, \beta_{n}, K_{n}}\left\{\left|S_{n} / n^{1-\kappa}+W_{n} / n^{1 / 2-\kappa}-n^{\kappa} m_{n}\right| \mid S_{n} / n+W_{n} / n^{1 / 2}>\delta m_{n}\right\} \\
& =\frac{1}{\int_{\mathbb{R}} \exp \left[-\frac{1}{2} g^{(2)}(\bar{x}) x^{2}\right] d x} \cdot \int_{\mathbb{R}}|x| \exp \left[-\frac{1}{2} g^{(2)}(\bar{x}) x^{2}\right] d x .
\end{aligned}
$$

Formula (7.2) is proved in two steps.

Step 1. Prove the second limit in (7.2). The proof is based on a representation formula, a weak convergent result, and a uniform integrability estimate. The verification of the second limit in (7.2) is the heart of the proof of part (b) of Theorem 6.1 and is motivated just below. The proof of the second limit in (7.2) is given in part (b) of Lemma 9.1.

Step 2. Prove the first limit in (7.2). This is done in two substeps, which we now explain.

Substep 2a. Define

$$
C_{n}=\tilde{E}_{n, \beta_{n}, K_{n}}\left\{\left|S_{n} / n^{1-\kappa}-n^{\kappa} m_{n}\right| \mid S_{n} / n>\delta m_{n}\right\}
$$

and

$$
D_{n}=\tilde{E}_{n, \beta_{n}, K_{n}}\left\{\left|S_{n} / n^{1-\kappa}+W_{n} / n^{1 / 2-\kappa}-n^{\kappa} m_{n}\right| \mid S_{n} / n>\delta m_{n}\right\}
$$

Substep $2 \mathrm{a}$ is to prove that $\lim _{n \rightarrow \infty}\left|C_{n}-D_{n}\right|=0$. This is certainly plausible since $C_{n}$ and $D_{n}$ differ only by $W_{n} / n^{1 / 2-\kappa}$ in the argument of the absolute value and since $W_{n} / n^{1 / 2-\kappa} \rightarrow 0$ with probability 1 . Substep $2 \mathrm{a}$ is proved in Lemma 9.3. 
Substep 2b. Define

$$
F_{n}=\tilde{E}_{n, \beta_{n}, K_{n}}\left\{\left|S_{n} / n^{1-\kappa}+W_{n} / n^{1 / 2-\kappa}-n^{\kappa} m_{n}\right| \mid S_{n} / n+W_{n} / n^{1 / 2}>\delta m_{n}\right\}
$$

Substep $2 b$ is to prove that

$$
\lim _{n \rightarrow \infty} D_{n}=\lim _{n \rightarrow \infty} F_{n}=\frac{1}{\int_{\mathbb{R}} \exp \left[-\frac{1}{2} g^{(2)}(\bar{x}) x^{2}\right] d x} \cdot \int_{\mathbb{R}}|x| \exp \left[-\frac{1}{2} g^{(2)}(\bar{x}) x^{2}\right] d x
$$

The limit of $F_{n}$ as $n \rightarrow \infty$ is calculated in Step 1. The first equality in the last display is certainly plausible since $D_{n}$ and $F_{n}$ differ only by $W_{n} / n^{1 / 2}$ in the conditioned event and $W_{n} / n^{1 / 2} \rightarrow 0$ with probability 1 . The proof of this equality, given in part (b) of Lemma 9.4, is much more subtle than the proof that $\lim _{n \rightarrow \infty}\left|C_{n}-D_{n}\right|=0$ in Substep 2a.

Having explained the logic of the proof of part (b) of Theorem 6.1, we now outline the proof of the second limit in (7.2). We start by applying the representation formula in Lemma 8.1. For any nonnegative measurable function $\varphi$, this transformation allows us to write

$$
\begin{aligned}
& \int_{\Lambda^{n} \times \Omega} \varphi\left(S_{n} / n^{1-\kappa}+W_{n} / n^{1 / 2-\kappa}\right) d\left(P_{n, \beta_{n}, K_{n}} \times Q\right) \\
& =\frac{1}{\int_{\mathbb{R}} \exp \left[-n G_{\beta_{n}, K_{n}}\left(x / n^{\kappa}\right)\right] d x} \cdot \int_{\mathbb{R}} \varphi(x) \exp \left[-n G_{\beta_{n}, K_{n}}\left(x / n^{\kappa}\right)\right] d x .
\end{aligned}
$$

In this formula $G_{\beta_{n}, K_{n}}$ is the free enery function defined in (2.3)-(2.4). Formula (7.3) is the conclusion of Lemma 8.1.

We now adapt this representation to the expected value in the second line of (7.2). For $\bar{\delta} \in(0,1)$ define

$$
A_{n}(\bar{\delta})=\left\{S_{n} / n+W_{n} / n^{1 / 2}>\bar{\delta} m_{n}\right\}=\left\{S_{n} / n^{1-\kappa}+W_{n} / n^{1 / 2-\kappa}>\bar{\delta} n^{\kappa} m_{n}\right\}
$$


Then the expected value in the second line of (7.2) equals

$$
\frac{1}{\tilde{E}_{n, \beta_{n}, K_{n}}\left\{1_{A_{n}(\bar{\delta})}\right\}} \cdot \tilde{E}_{n, \beta_{n}, K_{n}}\left\{\left|S_{n} / n^{1-\kappa}+W_{n} / n^{1 / 2-\kappa}-n^{\kappa} m_{n}\right| \cdot 1_{A_{n}(\bar{\delta})}\right\} .
$$

Let $Z_{n, \kappa}=\int_{\mathbb{R}} \exp \left[-n G_{\beta_{n}, K_{n}}\left(x / n^{\kappa}\right)\right] d x$. Since

$$
1_{A_{n}(\bar{\delta})}=1_{\left(n^{\kappa} \bar{\delta} m_{n}, \infty\right)}\left(S_{n} / n^{1-\kappa}+W_{n} / n^{1 / 2-\kappa}\right),
$$

we can apply (7.3) with $\varphi(x)=\left|x-n^{\kappa} m_{n}\right| 1_{\left(n^{\kappa} \bar{\delta} m_{n}, \infty\right)}(x)$ to write the numerator in (7.4) as

$$
\begin{aligned}
\int_{\Lambda^{n} \times \Omega} & \left|S_{n} / n^{1-\kappa}+W_{n} / n^{1 / 2-\kappa}-n^{\kappa} m_{n}\right| \cdot 1_{A_{n}(\bar{\delta})} d\left(P_{n, \beta_{n}, K_{n}} \times Q\right) \\
= & \frac{1}{Z_{n, \kappa}} \cdot \int_{\mathbb{R}}\left|x-n^{\kappa} m_{n}\right| \cdot 1_{\left(n^{\kappa} \bar{\delta} m_{n}, \infty\right)}(x) \exp \left[-n G_{\beta_{n}, K_{n}}\left(x / n^{\kappa}\right)\right] d x \\
= & \frac{1}{Z_{n, \kappa}} \cdot \int_{-n^{\kappa}(1-\bar{\delta}) m_{n}}^{\infty}|x| \exp \left[-n G_{\beta_{n}, K_{n}}\left(x / n^{\kappa}+m_{n}\right)\right] d x
\end{aligned}
$$

The denominator in (7.4) can be written in the same form with the absolute value function replaced by the constant function 1. Multiplying the numerator and denominator in (7.4) by the constant $\exp \left[G_{\beta_{n}, K_{n}}\left(m_{n}\right)\right]$, we see that the conditional expectation in the second line of (7.2) can be written as

$$
\begin{aligned}
\tilde{E}_{n, \beta_{n}, K_{n}}\left\{\left|S_{n} / n^{1-\kappa}+W_{n} / n^{1 / 2-\kappa}-n^{\kappa} m_{n}\right| \mid S_{n} / n+W_{n} / n^{1 / 2}>\delta m_{n}\right\} \\
=\frac{1}{\int_{-n^{\kappa}(1-\bar{\delta}) m_{n}}^{\infty} \exp \left[-n G_{\beta_{n}, K_{n}}\left(x / n^{\kappa}+m_{n}\right)+n G_{\beta_{n}, K_{n}}\left(m_{n}\right)\right] d x} \\
\quad \cdot \int_{-n^{\kappa}(1-\bar{\delta}) m_{n}}^{\infty}|x| \exp \left[-n G_{\beta_{n}, K_{n}}\left(x / n^{\kappa}+m_{n}\right)+n G_{\beta_{n}, K_{n}}\left(m_{n}\right)\right] d x
\end{aligned}
$$

The conclusion is that Step 1 in the proof of part (b) of Theorem 6.1 is proved by showing that for $h(x)=|x|$ and for $h \equiv 1$ 


$$
\begin{gathered}
\lim _{n \rightarrow \infty} \int_{-n^{\kappa}(1-\bar{\delta}) m_{n}}^{\infty} h(x) \exp \left[-n G_{\beta_{n}, K_{n}}\left(x / n^{\kappa}+m_{n}\right)+n G_{\beta_{n}, K_{n}}\left(m_{n}\right)\right] d x \\
=\int_{\mathbb{R}} h(x) \exp \left[-\frac{1}{2} g^{(2)}(\bar{x}) x^{2}\right] d x .
\end{gathered}
$$

To facilitate the calculation in (7.6), we reexpress it in terms of measures. For $n \in \mathbb{N}$ let $\Psi_{n}$ and $\Psi$ denote the measures on $\mathbb{R}$ defined by

$$
\Psi_{n}(d x)=1_{\left(-n^{\kappa}(1-\bar{\delta}) m_{n}, \infty\right)}(x) \cdot \exp \left[-n G_{\beta_{n}, K_{n}}\left(x / n^{\kappa}+m_{n}\right)+n G_{\beta_{n}, K_{n}}\left(m_{n}\right)\right] d x
$$

and

$$
\Psi(d x)=\exp \left[-\frac{1}{2} g^{(2)}(\bar{x}) x^{2}\right] d x
$$

Then the limits in (7.6) for $h(x)=|x|$ and $h(x) \equiv 1$ can be written as

$$
\lim _{n \rightarrow \infty} \int_{\mathbb{R}}|x| d \Psi_{n}=\int_{\mathbb{R}}|x| d \Psi \text { and } \lim _{n \rightarrow \infty} \int_{\mathbb{R}} 1 d \Psi_{n}=\int_{\mathbb{R}} 1 d \Psi
$$

We claim that the sequence $\Psi_{n}$ converges weakly to $\Psi$, meaning that for any bounded, continuous function $f$

$$
\lim _{n \rightarrow \infty} \int_{\mathbb{R}} f d \Psi_{n}=\int_{\mathbb{R}} f d \Psi
$$

This will be motivated in the next few paragraphs. Taking $f \equiv 1$ gives the second limit in (7.7). As discussed in Theorem 4 in $\S$ II.6 of [17], the standard way of extending (7.8) from bounded, continuous functions $f$ to the unbounded absolute function $|x|$ is to prove the uniform integrability estimate

$$
\lim _{j \rightarrow \infty} \sup _{n \in \mathbb{N}} \int_{\{|x|>j\}}|x| d \Psi_{n}=0 .
$$

In the present setting there is a condition weaker than uniform integrability that yields the same conclusion. As shown in [13, Prop. 8.3] and Proposition 9.2 in this dissertation, if

$$
\lim _{j \rightarrow \infty} \limsup _{n \rightarrow \infty} \int_{\{|x|>j\}}|x| d \Psi_{n}=0
$$


then $\int_{\mathbb{R}}|x| d \Psi_{n} \rightarrow \int_{\mathbb{R}}|x| d \Psi$. This is the first limit in (7.7). The verification of the limit in the last display is carried out in subsection 9.1.

We now motivate the limit

$$
\lim _{n \rightarrow \infty} \int_{\mathbb{R}} f d \Psi_{n}=\int_{\mathbb{R}} f d \Psi=\int_{\mathbb{R}} f(x) \exp \left[-\frac{1}{2} g^{(2)}(\bar{x}) x^{2}\right] d x
$$

for any bounded, continuous function $f$. By definition of $\Psi_{n}$

$$
\begin{array}{rl}
\int_{\mathbb{R}} f & d \Psi_{n} \\
\quad=\int_{\mathbb{R}} f(x) \cdot 1_{\left(-n^{\kappa}(1-\bar{\delta}) m_{n}, \infty\right)}(x) \cdot \exp \left[-n G_{\beta_{n}, K_{n}}\left(x / \kappa+m_{n}\right)+n G_{\beta_{n}, K_{n}}\left(m_{n}\right)\right] d x
\end{array}
$$

Formally, we motivate (7.10) by interchanging the limit with the integral and using the fact that, since $\bar{\delta} \in(0,1), 1_{\left(-n^{\kappa}(1-\bar{\delta}) m_{n}, \infty\right)}(x)$ has the limit 1 for each $x$. We obtain

$$
\begin{aligned}
\lim _{n \rightarrow \infty} & \int_{\mathbb{R}} f d \Psi_{n} \\
& =\int_{\mathbb{R}} f(x)\left(\lim _{n \rightarrow \infty} \exp \left[-n G_{\beta_{n}, K_{n}}\left(x / n^{\kappa}+m_{n}\right)+n G_{\beta_{n}, K_{n}}\left(m_{n}\right)\right]\right) d x .
\end{aligned}
$$

We complete the motivation of (7.10) by applying the fact proved in part (a) of Lemma 8.5 that

$$
\lim _{n \rightarrow \infty}\left(n G_{\beta_{n}, K_{n}}\left(x / n^{\kappa}+m_{n}\right)-n G_{\beta_{n}, K_{n}}\left(m_{n}\right)\right)=\frac{1}{2} g^{(2)}(\bar{x}) x^{2} .
$$

Given the limit in (7.13), the interchange of the limit with the integral in (7.12) must be justified. As shown in Lemma 8.7, we do this by appealing to two facts: first, the Dominated Convergence Theorem, which follows from Lemma 8.5, and second, the large deviation estimate given in Lemma 8.6. In order to prove Lemma 8.5, hypothesis (iii') in Theorem 4.1 is needed. In the statement of the conditional limit theorem in part (b) of Theorem 6.1 , there appears a quantity $\Delta \in(0,1)$; the number $\delta$ appearing in the conditioned set must satisfy $\delta \in(\Delta, 1)$. The choice of $\Delta$ is dictated by the proof of the lower bound in part (b) of Lemma 8.5. 


\subsection{Outline of the proof of part (a) of Theorem 6.1}

We now turn to the proof of the conditional central limit theorem in part (a) of Theorem 6.1. It follows the same pattern of proof as the conditional limit theorem in part (b) of Theorem 6.1, but is more straightforward. As we will see, like part (b) of Theorem 6.1, part (a) of that theorem is a consequence of the weak convergence limit stated in (7.10) and proved in Lemma 8.7.

In order to prove the conditional central limit theorem, we must prove that for any bounded, continuous function $f$

$$
\begin{aligned}
\lim _{n \rightarrow \infty} & E_{n, \beta_{n}, K_{n}}\left\{f\left(n^{\kappa}\left(S_{n} / n-m\left(\beta_{n}, K_{n}\right)\right)\right) \mid S_{n} / n>\delta m\left(\beta_{n}, K_{n}\right)\right\} \\
& =\lim _{n \rightarrow \infty} E_{n, \beta_{n}, K_{n}}\left\{f\left(S_{n} / n^{1-\kappa}-n^{\kappa} m\left(\beta_{n}, K_{n}\right)\right) \mid S_{n} / n>\delta m\left(\beta_{n}, K_{n}\right)\right\} \\
& =E\left\{f\left(N\left(0,1 / g^{(2)}(\bar{x})\right)\right)\right\} \\
& =\frac{1}{\int_{\mathbb{R}} \exp \left[-\frac{1}{2} g^{(2)}(\bar{x}) x^{2}\right] d x} \cdot \int_{\mathbb{R}} f(x) \exp \left[-\frac{1}{2} g^{(2)}(\bar{x}) x^{2}\right] d x .
\end{aligned}
$$

By the theory of weak convergence of measures, it suffices to prove this limit for any bounded, uniformly continuous function $f$ [17, Remark 1, p. 313].

We prove (7.14) by the analogous two steps used to prove the conditional limit theorem in part (b) of Theorem 6.1, replacing the absolute value function by the bounded, uniformly continuous function $f$. Step 1 is based on the representation formula in Lemma 8.1, which allows us to write

$$
\begin{aligned}
\tilde{E}_{n, \beta_{n}, K_{n}}\left\{f\left(S_{n} / n^{1-\kappa}+W_{n} / n^{1 / 2-\kappa}-n^{\kappa} m_{n}\right) \mid S_{n} / n+W_{n} / n^{1 / 2}>\delta m_{n}\right\} \\
=\frac{1}{\int_{-n^{\kappa}(1-\bar{\delta}) m_{n}}^{\infty} \exp \left[-n G_{\beta_{n}, K_{n}}\left(x / n^{\kappa}+m_{n}\right)+n G_{\beta_{n}, K_{n}}\left(m_{n}\right)\right] d x} \\
\quad \cdot \int_{-n^{\kappa}(1-\bar{\delta}) m_{n}}^{\infty} f(x) \exp \left[-n G_{\beta_{n}, K_{n}}\left(x / n^{\kappa}+m_{n}\right)+n G_{\beta_{n}, K_{n}}\left(m_{n}\right)\right] d x .
\end{aligned}
$$

The weak convergence limit (7.10) applied to the given $f$ and to $f \equiv 1$ shows that 


$$
\begin{aligned}
& \tilde{E}_{n, \beta_{n}, K_{n}}\left\{f\left(S_{n} / n^{1-\kappa}+W_{n} / n^{1 / 2-\kappa}-n^{\kappa} m_{n}\right) \mid S_{n} / n+W_{n} / n^{1 / 2}>\delta m_{n}\right\} \\
& =\frac{1}{\int_{\mathbb{R}} \exp \left[-\frac{1}{2} g^{(2)}(\bar{x}) x^{2}\right] d x} \cdot \int_{\mathbb{R}} f(x) \exp \left[-\frac{1}{2} g^{(2)}(\bar{x}) x^{2}\right] d x
\end{aligned}
$$

This completes Step 1 in the proof of part (a) of Theorem 6.1. We avoid the uniform integrability-type estimate (7.9), which is necessary for the unbounded absolute value function $|x|$.

Step 2 in the proof of part (a) of Theorem 6.1 is to show that

$$
\begin{aligned}
& \lim _{n \rightarrow \infty} E_{n, \beta_{n}, K_{n}}\left\{f\left(n^{\kappa}\left(S_{n} / n-m\left(\beta_{n}, K_{n}\right)\right)\right) \mid S_{n} / n>\delta m\left(\beta_{n}, K_{n}\right)\right\}= \\
& \quad \lim _{n \rightarrow \infty} \tilde{E}_{n, \beta_{n}, K_{n}}\left\{f\left(S_{n} / n^{1-\kappa}+W_{n} / n^{1 / 2-\kappa}-n^{\kappa} m_{n}\right) \mid S_{n} / n+W_{n} / n^{1 / 2}>\delta m_{n}\right\} .
\end{aligned}
$$

Like the proof of part (b) of Theorem 6.1, Step 2 is done in two substeps.

Define

$$
\tilde{C}_{n}=\tilde{E}_{n, \beta_{n}, K_{n}}\left\{f\left(S_{n} / n^{1-\kappa}-n^{\kappa} m_{n}\right) \mid S_{n} / n>\delta m_{n}\right\}
$$

and

$$
\tilde{D}_{n}=\tilde{E}_{n, \beta_{n}, K_{n}}\left\{f\left(S_{n} / n^{1-\kappa}+W_{n} / n^{1 / 2-\kappa}-n^{\kappa} m_{n}\right) \mid S_{n} / n>\delta m_{n}\right\}
$$

Substep 2a is to prove that $\lim _{n \rightarrow \infty}\left|\tilde{C}_{n}-\tilde{D}_{n}\right|=0$. In order to prove this, we write $\left|\tilde{C}_{n}-\tilde{D}_{n}\right|$ as a sum of an integral on the set where $\left|W_{n} / n^{1 / 2-\kappa}\right| \leq \xi$, for a suitable choice of $\xi>0$, and an integral on the set where $\left|W_{n} / n^{1 / 2-\kappa}\right|>\xi$. By choosing $\xi>0$ sufficiently small, the uniform continuity of $f$ guarantees that the first integral can be made arbitrarily small. Because there exists a constant $c>0$ such that for all $n \in \mathbb{N}, Q\left\{\left|W_{n} / n^{1 / 2-\kappa}\right|>\xi \mid\right\} \leq$ $\exp \left[-c n^{1-2 \kappa}\right]$, the second integral can also be made arbitrarily small for all sufficiently large $n$. Details are given in the proof of Lemma 10.2.

Substep $2 \mathrm{~b}$ is to prove that

$$
\lim _{n \rightarrow \infty} \tilde{D}_{n}=\lim _{n \rightarrow \infty} \tilde{F}_{n}=\frac{1}{\int_{\mathbb{R}} \exp \left[-\frac{1}{2} g^{(2)}(\bar{x}) x^{2}\right] d x} \cdot \int_{\mathbb{R}} f(x) \exp \left[-\frac{1}{2} g^{(2)}(\bar{x}) x^{2}\right] d x
$$


where

$$
\tilde{F}_{n}=\tilde{E}_{n, \beta_{n}, K_{n}}\left\{f\left(S_{n} / n^{1-\kappa}+W_{n} / n^{1 / 2-\kappa}-n^{\kappa} m_{n}\right) \mid S_{n} / n+W_{n} / n^{1 / 2}>\delta m_{n}\right\} .
$$

Thus $\tilde{F}_{n}$ is obtained from $\tilde{D}_{n}$ by replacing $S_{n} / n$ in the conditioned event $\left\{S_{n} / n>\delta m_{n}\right\}$ by $S_{n} / n+W_{n} / n^{1 / 2}$. The limit of $\tilde{F}_{n}$ as $n \rightarrow \infty$ is calculated in Step 1. We write $f=f^{+}-f^{-}$, where $f^{+}=\max (f, 0)$ and $f^{-}=\max (-f, 0)$. Substep $2 \mathrm{~b}$ is proved by following the pattern of proof of Substep $2 b$ in the proof of part (b) of Theorem 6.1. Specifically, we apply the calculations in the latter proof twice. The first time the absolute value function is replaced by $f^{+}$, and the second time the absolute value function is replaced by $f^{-}$. The details are given in Lemma 10.4.

Together, Step 1 and Substeps $2 \mathrm{a}$ and $2 \mathrm{~b}$ show that

$$
\begin{aligned}
\lim _{n \rightarrow \infty} \tilde{C}_{n} & =\lim _{n \rightarrow \infty} \tilde{D}_{n}=\lim _{n \rightarrow \infty} \tilde{F}_{n} \\
& =\frac{1}{\int_{\mathbb{R}} \exp \left[-\frac{1}{2} g^{(2)}(\bar{x}) x^{2}\right] d x} \cdot \int_{\mathbb{R}} f(x) \exp \left[-\frac{1}{2} g^{(2)}(\bar{x}) x^{2}\right] d x
\end{aligned}
$$

This is the limit (7.14). The outline of the proof of part (a) of Theorem 6.1 is now complete.

In the next section we prove a number of lemmas that will be used in section 9 to prove part (b) of Theorem 6.1. Several of them will be also used in section 10 to prove part (a) of Theorem 6.1. 


\section{CHAPTER 8}

\section{PREPARATORY LEMMAS FOR PROOF OF PART (B) OF THEOREM 6.1}

Let $\left(\beta_{n}, K_{n}\right)$ be a positive sequence. Throughout this section we work with $0<\alpha<\alpha_{0}$ and denote $m\left(\beta_{n}, K_{n}\right)$ by $m_{n}$. Let $W_{n}$ be a sequence of normal random variables with mean 0 and variance $\left(2 \beta_{n} K_{n}\right)^{-1}$ defined on a probability space $(\Omega, \mathcal{F}, Q)$. We denote by $\tilde{E}_{n, \beta_{n}, K_{n}}$ expectation with respect to the product measure $P_{n, \beta_{n}, K_{n}} \times Q ; P_{n, \beta_{n}, K_{n}}$ is defined in (2.1)-(2.2).

Lemma 8.1 is a representation formula that will be used to study the limit of the conditional expectation in the second line of (7.2). In particular, it is directly applied to prove Lemma 8.2, Lemma 8.6, and the MDP stated in Theorem 6.2. This lemma is proved in Appendix C.

Lemma 8.1. Given a positive sequence $\left(\beta_{n}, K_{n}\right)$, let $W_{n}$ be a sequence of normal random variables with mean 0 and variance $\left(2 \beta_{n} K_{n}\right)^{-1}$ defined on a probability space $(\Omega, \mathcal{F}, Q)$. Then for any $\bar{\gamma} \in[0,1)$ and any bounded, measurable function $\varphi$

$$
\begin{aligned}
& \int_{\Lambda^{n} \times \Omega} \varphi\left(S_{n} / n^{1-\bar{\gamma}}+W_{n} / n^{1 / 2-\bar{\gamma}}\right) d\left(P_{n, \beta_{n}, K_{n}} \times Q\right) \\
& \quad=\frac{1}{\int_{\mathbb{R}} \exp \left[-n G_{\beta_{n}, K_{n}}\left(x / n^{\bar{\gamma}}\right)\right] d x} \cdot \int_{\mathbb{R}} \varphi(x) \exp \left[-n G_{\beta_{n}, K_{n}}\left(x / n^{\bar{\gamma}}\right)\right] d x .
\end{aligned}
$$

In this formula $G_{\beta_{n}, K_{n}}$ is the free energy function defined in (2.4).

Lemma 8.2 uses the representation formula in the preceding lemma to rewrite the conditional expectation in the second line of (7.2). 
Lemma 8.2. We assume that $\left(\beta_{n}, K_{n}\right)$ satisfies the hypotheses of Theorem 4.1 for all $0<$ $\alpha<\alpha_{0}$. For any $\bar{\delta} \in(0,1)$ define

$$
A_{n}(\bar{\delta})=\left\{S_{n} / n+W_{n} / n^{1 / 2}>\bar{\delta} m_{n}\right\}
$$

where $m_{n}=m\left(\beta_{n}, K_{n}\right)$. Given any $\alpha \in\left(0, \alpha_{0}\right)$, define $\kappa=\frac{1}{2}\left(1-\alpha / \alpha_{0}\right)+\theta \alpha$. The following conclusions hold.

(a) For any bounded, measurable function $h$

$$
\begin{aligned}
& \tilde{E}_{n, \beta_{n}, K_{n}}\left\{h\left(S_{n} / n^{1-\kappa}+W_{n} / n^{1 / 2-\kappa}-n^{\kappa} m_{n}\right) \cdot 1_{A_{n}(\bar{\delta})}\right\} \\
& =\frac{1}{Z_{n, \kappa}} \int_{-n^{\kappa}(1-\bar{\delta}) m_{n}}^{\infty} h(x) \exp \left[-n G_{\beta_{n}, K_{n}}\left(x / n^{\kappa}+m_{n}\right)\right] d x
\end{aligned}
$$

where $Z_{n, \kappa}=\int_{\mathbb{R}} \exp \left[-n G_{\beta_{n}, K_{n}}\left(x / n^{\kappa}\right)\right] d x$. In particular, if $h \equiv 1$, then

$$
\begin{aligned}
\tilde{E}_{n, \beta_{n}, K_{n}}\left\{1_{A_{n}(\bar{\delta})}\right\} & =\left(P_{n, \beta_{n}, K_{n}} \times Q\right)\left\{A_{n}(\bar{\delta})\right\} \\
& =\frac{1}{Z_{n, \kappa}} \int_{-n^{\kappa}(1-\bar{\delta}) m_{n}}^{\infty} \exp \left[-n G_{\beta_{n}, K_{n}}\left(x / n^{\kappa}+m_{n}\right)\right] d x
\end{aligned}
$$

(b) We have the representation

$$
\begin{aligned}
\tilde{E}_{n, \beta_{n}, K_{n}}\left\{\left|S_{n} / n^{1-\kappa}+W_{n} / n^{1 / 2-\kappa}-n^{\kappa} m_{n}\right| \mid A_{n}(\bar{\delta})\right\} \\
=\frac{1}{\int_{-n^{\kappa}(1-\bar{\delta}) m_{n}}^{\infty} \exp \left[-n G_{\beta_{n}, K_{n}}\left(x / n^{\kappa}+m_{n}\right)+n G_{\beta_{n}, K_{n}}\left(m_{n}\right)\right] d x} \\
\quad \cdot \int_{-n^{\kappa}(1-\bar{\delta}) m_{n}}^{\infty}|x| \exp \left[-n G_{\beta_{n}, K_{n}}\left(x / n^{\kappa}+m_{n}\right)+n G_{\beta_{n}, K_{n}}\left(m_{n}\right)\right] d x
\end{aligned}
$$

Proof. (a) By part (c) of Theorem 6.1, $\kappa \in\left(\theta \alpha_{0}, 1 / 2\right) \subset[0,1)$. We apply Lemma 8.1 with $\varphi(x)=h\left(x-n^{\kappa} m_{n}\right) \cdot 1_{\left(n^{\kappa} \bar{\delta} m_{n}, \infty\right)}(x)$ and $\bar{\gamma}=\kappa$, obtaining 


$$
\begin{aligned}
\tilde{E}_{n, \beta_{n}, K_{n}}\left\{h\left(S_{n} / n^{1-\kappa}+W_{n} / n^{1 / 2-\kappa}-n^{\kappa} m_{n}\right) \cdot 1_{\left\{S_{n} / n^{1-\kappa}+W_{n} / n^{1 / 2-\kappa}>n^{\kappa} \bar{\delta} m_{n}\right\}}\right\} \\
=\int_{\Lambda^{n} \times \Omega} h\left(S_{n} / n^{1-\kappa}+W_{n} / n^{1 / 2-\kappa}-n^{\kappa} m_{n}\right) \cdot 1_{\left\{S_{n} / n^{1-\kappa}+W_{n} / n^{1 / 2-\kappa}>n^{\kappa} \bar{\delta} m_{n}\right\}} d\left(P_{n, \beta_{n}, K_{n}} \times Q\right) \\
=\frac{1}{\int_{\mathbb{R}} \exp \left[-n G_{\beta_{n}, K_{n}}\left(x / n^{\kappa}\right)\right] d x} \cdot \int_{\mathbb{R}} h\left(x-n^{\kappa} m_{n}\right) \cdot 1_{\left(n^{\kappa} \bar{\delta} m_{n}, \infty\right)}(x) \exp \left[-n G_{\beta_{n}, K_{n}}\left(x / n^{\kappa}\right)\right] d x \\
=\frac{1}{Z_{n, \kappa}} \cdot \int_{-n^{\kappa}(1-\bar{\delta}) m_{n}}^{\infty} h(x) \exp \left[-n G_{\beta_{n}, K_{n}}\left(x / n^{\kappa}+m_{n}\right)\right] d x .
\end{aligned}
$$

This yields (8.1). Formula (8.2) follows by taking $h \equiv 1$.

(b) We apply part (a) to the sequence of bounded, measurable functions $h_{j}(x)=|x| \wedge j$, $j \in \mathbb{N}$. By the monotone convergence theorem we obtain (8.1) with $h(x)$ replaced by $|x|$. Part (b) now follows by using the definition of conditional expectation and multiplying the numerator and denominator of the resulting fraction by $\exp \left[n G_{\beta_{n}, K_{n}}\left(m_{n}\right)\right]$. The proof of Lemma 8.2 is complete.

Lemma 8.3 gives the asymptotic behavior of $G_{\beta_{n}, K_{n}}\left(m_{n}\right)$. This lemma is used to prove Lemma 8.4 and removes an error term that arises in the proof of part (a) of Lemma 9.1.

Lemma 8.3. We assume that $\left(\beta_{n}, K_{n}\right)$ satisfies the hypotheses of Theorem 4.1 for all $0<$ $\alpha<\alpha_{0}$. Let $m_{n}=m\left(\beta_{n}, K_{n}\right)$. Then for all $0<\alpha<\alpha_{0}$,

$$
\lim _{n \rightarrow \infty} n^{\alpha / \alpha_{0}} G_{\beta_{n}, K_{n}}\left(m_{n}\right)=g(\bar{x})<0
$$

Proof. We have

$$
\begin{aligned}
\left|n^{\alpha / \alpha_{0}} G_{\beta_{n}, K_{n}}\left(m_{n}\right)-g(\bar{x})\right| \leq & \left|n^{\alpha / \alpha_{0}} G_{\beta_{n}, K_{n}}\left(n^{\theta \alpha} m_{n} / n^{\theta \alpha}\right)-g\left(n^{\theta \alpha} m_{n}\right)\right| \\
& +\left|g\left(n^{\theta \alpha} m_{n}\right)-g(\bar{x})\right| .
\end{aligned}
$$

The hypotheses of Theorem 3.1 for all $0<\alpha<\alpha_{0}$ consist of a subset of the hypotheses of Theorem 4.1. By hypothesis (iii)(a) of Theorem 3.1

$$
\lim _{n \rightarrow \infty} n^{\alpha / \alpha_{0}} G_{\beta_{n}, K_{n}}\left(x / n^{\theta \alpha}\right)=g(x)
$$


uniformly for $x$ in compact subsets of $\mathbb{R}$. According to Theorem 3.1, $n^{\theta \alpha} m_{n} \rightarrow \bar{x}$, and so for any $\varepsilon>0$ the sequence $n^{\theta \alpha} m_{n}$ lies in the compact set $[\bar{x}-\varepsilon, \bar{x}+\varepsilon]$ for all sufficiently large $n$. Setting $x=n^{\theta \alpha} m_{n}$, we see that the first term on the right-hand side of (8.4) has the limit 0 . Because of the limit $n^{\theta \alpha} m_{n} \rightarrow \bar{x}$ and the continuity of $g$, the second term on the right-hand side of (8.4) also converges to 0 as $n \rightarrow \infty$. It follows that

$$
\lim _{n \rightarrow \infty}\left|n^{\alpha / \alpha_{0}} G_{\beta_{n}, K_{n}}\left(m_{n}\right)-g(\bar{x})\right|=0 .
$$

By hypothesis (iii)(b) of Theorem 3.1, $\bar{x}>0$ is the unique nonnegative, global minimum point of $g$. Thus

$$
g(\bar{x})<g(0)=\lim _{n \rightarrow \infty} n^{\alpha / \alpha_{0}} G_{\beta_{n}, K_{n}}(0)=0 .
$$

The proof of lemma is complete.

Lemma 8.4 gives an inequality involving $n G_{\beta_{n}, K_{n}}\left(m_{n}\right)$ and the quantity $Z_{n, \kappa}$ defined in part (a) of Lemma 8.2. This inequality is used in the proof of Lemma 8.6 and the proof of part (a) of Lemma 9.4.

Lemma 8.4. We assume that $\left(\beta_{n}, K_{n}\right)$ satisfies the hypotheses of Theorem 4.1 for all $0<$ $\alpha<\alpha_{0}$. For any $0<\alpha<\alpha_{0}$ define $\kappa=\frac{1}{2}\left(1-\alpha / \alpha_{0}\right)+\theta \alpha$ and

$$
Z_{n, \kappa}=\int_{\mathbb{R}} \exp \left[-n G_{\beta_{n}, K_{n}}\left(x / n^{\kappa}\right)\right] d x
$$

Let $m_{n}=m\left(\beta_{n}, K_{n}\right)$. Then for any $\varepsilon>0$ and all sufficiently large $n$

$$
\exp \left[n G_{\beta_{n}, K_{n}}\left(m_{n}\right)\right] \cdot Z_{n, \kappa} \leq \exp \left[\varepsilon n^{1-\alpha / \alpha_{0}}\right] .
$$


Proof. For any $0<\alpha<\alpha_{0}$ define

$$
Z_{n, \theta \alpha}=\int_{\mathbb{R}} \exp \left[-n G_{\beta_{n}, K_{n}}\left(x / n^{\theta \alpha}\right)\right] d x
$$

Changing variables shows that $Z_{n, \kappa}=n^{\kappa-\theta \alpha} Z_{n, \theta \alpha}$. The MDP stated in Theorem 6.2 is proved in appendix B via an associated Laplace principle. A key step in this proof is the limit

$$
\lim _{n \rightarrow \infty} \frac{1}{n^{1-\alpha / \alpha_{0}}} \log \int_{\mathbb{R}} \exp \left[n^{1-\alpha / \alpha_{0}} \psi(x)-n G_{\beta_{n}, K_{n}}\left(x / n^{\theta \alpha}\right)\right] d x=\sup _{x \in \mathbb{R}}\{\psi(x)-g(x)\}
$$

where $\psi$ is any bounded, continuous function mapping $\mathbb{R}$ to $\mathbb{R}$. This is proved in (B.10) in appendix B with $u=\left(1-\alpha / \alpha_{0}\right)$ and $\gamma=\theta \alpha$. Setting $\psi=0$ gives the limit

$$
\lim _{n \rightarrow \infty} \frac{1}{n^{1-\alpha / \alpha_{0}}} \log Z_{n, \theta \alpha}=\lim _{n \rightarrow \infty} \frac{1}{n^{1-\alpha / \alpha_{0}}} \log \int_{\mathbb{R}} \exp \left[-n G_{\beta_{n}, K_{n}}\left(x / n^{\theta \alpha}\right)\right] d x=-\inf _{y \in \mathbb{R}} g(y)
$$

Since $Z_{n, \kappa}=n^{\kappa-\theta \alpha} Z_{n, \theta \alpha}$ and $g$ has a unique positive, global minimum point at $\bar{x}$,

$$
\begin{aligned}
\lim _{n \rightarrow \infty} \frac{1}{n^{1-\alpha / \alpha_{0}}} \log Z_{n, \kappa} & =\lim _{n \rightarrow \infty} \frac{1}{n^{1-\alpha / \alpha_{0}}} \log \left(n^{\kappa-\theta \alpha} Z_{n, \theta \alpha}\right) \\
& =-\inf _{y \in \mathbb{R}} g(y)=-g(\bar{x}) .
\end{aligned}
$$

By Lemma $8.3 \lim _{n \rightarrow \infty} n^{\alpha / \alpha_{0}} G_{\beta_{n}, K_{n}}\left(m_{n}\right)=g(\bar{x})$. Hence the asymptotic behaviors of $\log Z_{n, \kappa}$ and $n G_{\beta_{n}, K_{n}}\left(m_{n}\right)$ are related by

$$
\lim _{n \rightarrow \infty} \frac{1}{n^{1-\alpha / \alpha_{0}}} \log Z_{n, \kappa}=-\lim _{n \rightarrow \infty} \frac{1}{n^{1-\alpha / \alpha_{0}}} n G_{\beta_{n}, K_{n}}\left(m_{n}\right)
$$

Thus for any $\varepsilon>0$ and all sufficiently large $n$

$$
\frac{1}{n^{1-\alpha / \alpha_{0}}} \log Z_{n, \kappa}+\frac{1}{n^{1-\alpha / \alpha_{0}}} n G_{\beta_{n}, K_{n}}\left(m_{n}\right) \leq \varepsilon
$$


or equivalently

$$
\exp \left[n G_{\beta_{n}, K_{n}}\left(m_{n}\right)\right] \cdot Z_{n, \kappa} \leq \exp \left(\varepsilon n^{1-\alpha / \alpha_{0}}\right) .
$$

The proof of Lemma 8.4 is complete.

We recall that Step 1 in the proof of part (b) of Theorem 6.1 is to prove the second limit in (7.2):

$$
\begin{gathered}
\lim _{n \rightarrow \infty} \tilde{E}_{n, \beta_{n}, K_{n}}\left\{\left|S_{n} / n^{1-\kappa}+W_{n} / n^{1 / 2-\kappa}-n^{\kappa} m_{n}\right| \mid S_{n} / n+W_{n} / n^{1 / 2}>\delta m_{n}\right\} \\
=\frac{1}{\int_{\mathbb{R}} \exp \left[-\frac{1}{2} g^{(2)}(\bar{x}) x^{2}\right] d x} \cdot \int_{\mathbb{R}}|x| \exp \left[-\frac{1}{2} g^{(2)}(\bar{x}) x^{2}\right] d x .
\end{gathered}
$$

By part (b) of Lemma 8.2 the limit of the conditional expectation equals the limit of the product in the last two lines of $(8.3)$ with $\bar{\delta}=\delta$. For $\delta \in(0,1)$ this product has the form

$$
\begin{aligned}
& \frac{1}{\int_{-n^{\kappa}(1-\delta) m_{n}}^{\infty} \exp \left[-n G_{\beta_{n}, K_{n}}\left(x / n^{\kappa}+m_{n}\right)+n G_{\beta_{n}, K_{n}}\left(m_{n}\right)\right] d x} \\
& \cdot \int_{-n^{\kappa}(1-\delta) m_{n}}^{\infty}|x| \exp \left[-n G_{\beta_{n}, K_{n}}\left(x / n^{\kappa}+m_{n}\right)+n G_{\beta_{n}, K_{n}}\left(m_{n}\right)\right] d x .
\end{aligned}
$$

The calculation of the limit of this product depends in part on Lemma 8.7, which will be proved via the Dominated Convergence Theorem (DCT). Two key estimates are given in the next lemma. Part (b) of the next lemma also removes an error term that arises in the proof of part (a) of Lemma 9.1. The proof of Lemma 8.5 is postponed until the end of this section.

Lemma 8.5. We assume that $\left(\beta_{n}, K_{n}\right)$ satisfies the hypotheses of Theorem 4.1 for all $0<$ $\alpha<\alpha_{0}$. For any $0<\alpha<\alpha_{0}$ define $\kappa=\frac{1}{2}\left(1-\alpha / \alpha_{0}\right)+\theta \alpha$, and let $m_{n}=m\left(\beta_{n}, K_{n}\right)$. The following conclusions hold. 
(a) For all $x \in \mathbb{R}$

$$
\lim _{n \rightarrow \infty}\left(n G_{\beta_{n}, K_{n}}\left(x / n^{\kappa}+m_{n}\right)-n G_{\beta_{n}, K_{n}}\left(m_{n}\right)\right)=\frac{1}{2} g^{(2)}(\bar{x}) x^{2} .
$$

(b) There exists $\Delta \in(0,1)$ such that for any $\bar{\delta} \in(\Delta, 1)$ there exists $R>0$ such that for all sufficiently large $n$ and all $x \in \mathbb{R}$ satisfying $\left|x / n^{\kappa}\right|<R$ and $x / n^{\kappa}>-(1-\bar{\delta}) m_{n}$

$$
n G_{\beta_{n}, K_{n}}\left(x / n^{\kappa}+m_{n}\right)-n G_{\beta_{n}, K_{n}}\left(m_{n}\right) \geq \frac{1}{8} g^{(2)}(\bar{x}) x^{2}
$$

The next lemma removes an error term that arises in applying the DCT to prove Lemma 8.7. The next lemma also removes an error term that arises in the proof of part (a) of Lemma 9.1.

Lemma 8.6. We assume that $\left(\beta_{n}, K_{n}\right)$ satisfies the hypotheses of Theorem 4.1 for all $0<$ $\alpha<\alpha_{0}$. Then there exist a constant $c_{2}>0$ such that for all sufficiently large $n$

$$
\int_{R n^{\kappa}}^{\infty} \exp \left[-n G_{\beta_{n}, K_{n}}\left(x / n^{\kappa}+m_{n}\right)+n G_{\beta_{n}, K_{n}}\left(m_{n}\right)\right] d x \leq \exp \left[-c_{2} n\right] \rightarrow 0 \text { as } n \rightarrow \infty
$$

where $R$ is chosen as in part (b) of Lemma 8.5 and $m_{n}=m\left(\beta_{n}, K_{n}\right)$.

Proof. We start by applying Lemma 8.1 with $\varphi(x)=1_{\left(R n^{\kappa}+n^{\kappa} m_{n}, \infty\right)}(x)$ and $\bar{\gamma}=\kappa$, obtaining

$$
\begin{aligned}
& \left(P_{n, \beta_{n}, K_{n}} \times Q\right)\left\{S_{n} / n+W_{n} / n^{1 / 2} \geq R+m_{n}\right\} \\
& \quad=\left(P_{n, \beta_{n}, K_{n}} \times Q\right)\left\{S_{n} / n^{1-\kappa}+W_{n} / n^{1 / 2-\kappa} \geq R n^{\kappa}+m_{n} n^{\kappa}\right\} \\
& =\int_{\Lambda^{n} \times \Omega} 1_{\left\{S_{n} / n^{1-\kappa}+W_{n} / n^{1 / 2-\kappa} \geq R n^{\kappa}+m_{n} n^{\kappa}\right\}} d\left(P_{n, \beta_{n}, K_{n}} \times Q\right) \\
& =\frac{1}{Z_{n, \kappa}} \cdot \int_{\mathbb{R}} 1_{\left[R n^{\kappa}+m_{n} n^{\kappa}, \infty\right)}(x) \exp \left[-n G_{\beta_{n}, K_{n}}\left(x / n^{\kappa}\right)\right] d x \\
& =\frac{1}{Z_{n, \kappa}} \cdot \int_{R n^{\kappa}}^{\infty} \exp \left[-n G_{\beta_{n}, K_{n}}\left(x / n^{\kappa}+m_{n}\right)\right] d x,
\end{aligned}
$$


where $Z_{n, \kappa}=\int_{\mathbb{R}} \exp \left[-n G_{\beta_{n}, K_{n}}\left(x / n^{\kappa}\right)\right] d x$. Thus we have

$$
\begin{aligned}
& \int_{R n^{\kappa}}^{\infty} \exp \left[-n G_{\beta_{n}, K_{n}}\left(x / n^{\kappa}+m_{n}\right)+n G_{\beta_{n}, K_{n}}\left(m_{n}\right)\right] d x \\
& \quad=\exp \left[n G_{\beta_{n}, K_{n}}\left(m_{n}\right)\right] \cdot Z_{n, \kappa} \cdot\left(P_{n, \beta_{n}, K_{n}} \times Q\right)\left\{S_{n} / n+W_{n} / n^{1 / 2} \geq R+m_{n}\right\} \\
& \quad \leq \exp \left[n G_{\beta_{n}, K_{n}}\left(m_{n}\right)\right] \cdot Z_{n, \kappa} \cdot\left(P_{n, \beta_{n}, K_{n}} \times Q\right)\left\{S_{n} / n+W_{n} / n^{1 / 2} \geq R\right\} .
\end{aligned}
$$

By part (b) of Lemma 4.4 in [8], with respect to $P_{n, \beta_{n}, K_{n}} \times Q, S_{n} / n+W_{n} / n^{1 / 2}$ satisfies the large deviation principle on $\mathbb{R}$ with exponential speed $n$ and rate function $G_{\beta, K(\beta)}$. In particular, for the closed set $[R, \infty)$ we have the large deviation upper bound

$$
\limsup _{n \rightarrow \infty} \frac{1}{n} \log \left(P_{n, \beta_{n}, K_{n}} \times Q\right)\left\{S_{n} / n+W_{n} / n^{1 / 2} \geq R\right\} \leq-\inf _{x \geq R} G_{\beta, K(\beta)}(x) .
$$

By part (a) of Theorem 2.1, since $0<\beta \leq \beta_{c}$, we have $\mathcal{M}_{\beta, K(\beta)}=\{0\}$. Thus $G_{\beta, K(\beta)}$ has a unique global minimum point at 0 . Since $R>0$, it follows that

$$
\inf _{x \geq R} G_{\beta, K(\beta)}(x)>\inf _{x \in \mathbb{R}} G_{\beta, K(\beta)}(x)=0 .
$$

Therefore for all sufficiently large $n$

$$
\left(P_{n, \beta_{n}, K_{n}} \times Q\right)\left\{S_{n} / n+W_{n} / n^{1 / 2} \geq R\right\} \leq \exp \left[-c_{1} n\right]
$$

where $c_{1}=\inf _{x \geq R} G_{\beta, K(\beta)}(x) / 2>0$. We now appeal to Lemma 8.4, which states that for any $\varepsilon>0$ and all sufficiently large $n$

$$
\exp \left[n G_{\beta_{n}, K_{n}}\left(m_{n}\right)\right] \cdot Z_{n, \kappa} \leq \exp \left[\varepsilon n^{1-\alpha / \alpha_{0}}\right] .
$$


Since $0<1-\alpha / \alpha_{0}<1$, it follows that for all sufficiently large $n$

$$
\begin{aligned}
& \int_{R n^{\kappa}}^{\infty} \exp \left[-n G_{\beta_{n}, K_{n}}\left(x / n^{\kappa}+m_{n}\right)+n G_{\beta_{n}, K_{n}}\left(m_{n}\right)\right] d x \\
& \quad \leq \exp \left[n G_{\beta_{n}, K_{n}}\left(m_{n}\right)\right] \cdot Z_{n, \kappa} \cdot\left(P_{n, \beta_{n}, K_{n}} \times Q\right)\left\{S_{n} / n+W_{n} / n^{1 / 2} \geq R\right\} \\
& \quad \leq \exp \left[\varepsilon n^{1-\alpha / \alpha_{0}}\right] \cdot \exp \left[-c_{1} n\right] \\
& \quad \leq \exp \left[-c_{1} n / 2\right] .
\end{aligned}
$$

This gives the conclusion of Lemma 8.6 with $c_{2}=c_{1} / 2$. The proof of the lemma is complete.

Lemma 8.7 is a key result in the proof of the conditional limit stated in part (b) of Theorem 6.1. The lemma deals with limits needed in the proof of part (a) of Lemma 9.1. Lemma 8.7 is also used with $f \equiv 1$ in the proof of part (b) of Lemma 9.1 and part (a) of Lemma 9.4.

Lemma 8.7. We assume that $\left(\beta_{n}, K_{n}\right)$ satisfies the hypotheses of Theorem 4.1 for all $0<$ $\alpha<\alpha_{0}$. Given any $\alpha \in\left(0, \alpha_{0}\right)$, define $\kappa=\frac{1}{2}\left(1-\alpha / \alpha_{0}\right)+\theta \alpha$ and

$$
Z_{n, \kappa}=\int_{\mathbb{R}} \exp \left[-n G_{\beta_{n}, K_{n}}\left(x / n^{\kappa}\right)\right] d x
$$

For $\bar{\delta} \in(0,1)$ define

$$
A_{n}(\bar{\delta})=\left\{S_{n} / n+W_{n} / n^{1 / 2}>\bar{\delta} m_{n}\right\}
$$

where $m_{n}=m\left(\beta_{n}, K_{n}\right)$. Let $f$ be any bounded, continuous function and let $\Delta \in(0,1)$ be the number determined in part (b) of Lemma 8.5. Then for any $0<\alpha<\alpha_{0}$ and any $\bar{\delta} \in(\Delta, 1)$ we have the limit 


$$
\begin{aligned}
\lim _{n \rightarrow \infty} & \exp \left[n G_{\beta_{n}, K_{n}}\left(m_{n}\right)\right] \cdot Z_{n, \kappa} \cdot \tilde{E}_{n, \beta_{n}, K_{n}}\left\{f\left(S_{n} / n^{1-\kappa}+W_{n} / n^{1 / 2-\kappa}-n^{\kappa} m_{n}\right) \cdot 1_{A_{n}(\bar{\delta})}\right\} \\
& =\lim _{n \rightarrow \infty} \int_{-n^{\kappa}(1-\bar{\delta}) m_{n}}^{\infty} f(x) \exp \left[-n G_{\beta_{n}, K_{n}}\left(x / n^{\kappa}+m_{n}\right)+n G_{\beta_{n}, K_{n}}\left(m_{n}\right)\right] d x \\
& =\int_{\mathbb{R}} f(x) \exp \left[-\frac{1}{2} g^{(2)}(\bar{x}) x^{2}\right] d x .
\end{aligned}
$$

Proof. The first equality follows by applying part (a) of Lemma 8.2 to $h=f$. Concerning the second equality, we denote by $I_{n}$ the integral in the second line of (8.5). We write $I_{n}=I_{n}^{1}+I_{n}^{2}$, where

$$
I_{n}^{1}=\int_{-n^{\kappa}(1-\bar{\delta}) m_{n}}^{R n^{\kappa}} f(x) \exp \left[-n G_{\beta_{n}, K_{n}}\left(x / n^{\kappa}+m_{n}\right)+n G_{\beta_{n}, K_{n}}\left(m_{n}\right)\right] d x
$$

and

$$
I_{n}^{2}=\int_{R n^{\kappa}}^{\infty} f(x) \exp \left[-n G_{\beta_{n}, K_{n}}\left(x / n^{\kappa}+m_{n}\right)+n G_{\beta_{n}, K_{n}}\left(m_{n}\right)\right] d x .
$$

The number $R$ is chosen as in part (b) of Lemma 8.5. Since $f$ is bounded, Lemma 8.6 implies that there exists $c_{2}>0$ such that for all sufficiently large $n$

$$
\begin{aligned}
I_{n}^{2} & \leq\|f\|_{\infty} \int_{R n^{\kappa}}^{\infty} \exp \left[-n G_{\beta_{n}, K_{n}}\left(x / n^{\kappa}+m_{n}\right)+n G_{\beta_{n}, K_{n}}\left(m_{n}\right)\right] d x \\
& \leq\|f\|_{\infty} \exp \left[-c_{2} n\right] \rightarrow 0 \text { as } n \rightarrow \infty .
\end{aligned}
$$

Thus $I_{n}^{2} \rightarrow 0$ as $n \rightarrow \infty$.

Define

$$
h_{n}(x)=f(x) \exp \left[-n G_{\beta_{n}, K_{n}}\left(x / n^{\kappa}+m_{n}\right)+n G_{\beta_{n}, K_{n}}\left(m_{n}\right)\right]
$$

and

$$
h(x)=f(x) \exp \left[-\frac{1}{2} g^{(2)}(\bar{x}) x^{2}\right] .
$$


By part (a) of Lemma 8.5, $h_{n}(x) \rightarrow h(x)$ for all $x \in \mathbb{R}$. In addition, by part (b) of Lemma 8.5 , if $x \in\left(-(1-\bar{\delta}) m_{n} n^{\kappa}, R n^{\kappa}\right)$, then for all sufficiently large $n$

$$
n G_{\beta_{n}, K_{n}}\left(x / n^{\kappa}+m_{n}\right)-n G_{\beta_{n}, K_{n}}\left(m_{n}\right) \geq H(x)=\frac{1}{8} g^{(2)}(\bar{x}) x^{2} .
$$

Since $\exp [-H(x)]$ is integrable, the Dominated Convergence Theorem implies that

$$
\lim _{n \rightarrow \infty} I_{n}^{1}=\lim _{n \rightarrow \infty} \int_{-n^{\kappa}(1-\bar{\delta}) m_{n}}^{R n^{\kappa}} h_{n}(x) d x=\int_{\mathbb{R}} h(x) d x=\int_{\mathbb{R}} f(x) \exp \left[-\frac{1}{2} g^{(2)}(\bar{x}) x^{2}\right] d x .
$$

We conclude that

$$
\lim _{n \rightarrow \infty} I_{n}=\lim _{n \rightarrow \infty} I_{n}^{1}+\lim _{n \rightarrow \infty} I_{n}^{2}=\int_{\mathbb{R}} f(x) \exp \left[-\frac{1}{2} g^{(2)}(\bar{x}) x^{2}\right] d x
$$

This completes the proof of Lemma 8.7.

The next lemma collects several elementary but useful facts concerning the normal random variables $W_{n}$.

Lemma 8.8. Let $\left(\beta_{n}, K_{n}\right)$ be a positive sequence that converges either to a second-order point $(\beta, K(\beta)), 0<\beta<\beta_{c}$, or to the tricritical point $\left(\beta_{c}, K\left(\beta_{c}\right)\right)$. Let $W_{n}$ be a sequence of normal random variables with mean 0 and variance $\sigma_{n}^{2}=\left(2 \beta_{n} K_{n}\right)^{-1}$ defined on a probability space $(\Omega, \mathcal{F}, Q)$. The following conclusions hold.

(a) For $b>0$ and $\zeta>0$ there exists a constant $c>0$ such that for all $n, Q\left\{\left|W_{n}\right|>\right.$ $\left.b n^{\zeta}\right\} \leq \exp \left[-c n^{2 \zeta}\right]$.

(b) There exist a constant $c_{1}>0$ such that for all $n$

$$
\int_{\Omega}\left|W_{n}\right|^{2} d Q \leq c_{1} \text { and } \int_{\Omega}\left|W_{n}\right| d Q \leq \sqrt{c_{1}}
$$


Proof. (a) We have the bound

$$
Q\left\{\left|W_{n}\right|>b n^{\zeta}\right\}=\frac{\sqrt{2}}{\sqrt{\pi} \sigma_{n}} \int_{b n \zeta}^{\infty} \exp \left[-x^{2} / 2 \sigma_{n}^{2}\right] d x \leq \frac{\sqrt{2} \sigma_{n}}{\sqrt{\pi} b n^{\zeta}} \exp \left[-b^{2} n^{2 \zeta} / 2 \sigma_{n}^{2}\right]
$$

Part (a) now follows from the fact that since $\left(\beta_{n}, K_{n}\right)$ is a positive sequence converging to $(\beta, K(\beta))$ for $0<\beta \leq \beta_{c}$, the positive sequences $\sigma_{n}$ and $\sigma_{n}^{2}$ are bounded.

(b) Since $\int_{\Omega}\left|W_{n}\right|^{2} d Q=\sigma_{n}^{2}$ and $\int_{\Omega}\left|W_{n}\right| d Q \leq\left(\int_{\Omega}\left|W_{n}\right|^{2} d Q\right)^{1 / 2}=\sigma_{n}$, this follows from the fact that the positive sequences $\sigma_{n}^{2}$ and $\sigma_{n}$ are bounded. The proof of the lemma is complete.

The next lemma is used in the proof of part (a) of Lemma 9.4 and in the proof of part (a) of Lemma 10.4. Under the hypotheses of Theorem 4.1, for any $0<\alpha<\alpha_{0}$ the interval $\left(0, \frac{1}{2}-\theta \alpha\right)$ appearing in the next lemma is nonempty because by hypothesis (iii') $\frac{1}{2}-\theta \alpha>\frac{1}{2}-\theta \alpha_{0}>0$.

Lemma 8.9. We assume that $\left(\beta_{n}, K_{n}\right)$ satisfies the hypotheses of Theorem 4.1 for all $0<$ $\alpha<\alpha_{0}$. For $\bar{\delta} \in(0,1)$ define

$$
A_{n}(\bar{\delta})=\left\{S_{n} / n+W_{n} / n^{1 / 2}>\bar{\delta} m_{n}\right\}
$$

where $m_{n}=m\left(\beta_{n}, K_{n}\right)$. Let $\Delta \in(0,1)$ be the number determined in part (b) of Lemma 8.5. Assume that $0<\alpha<\alpha_{0}$ and choose any numbers $\delta_{1}, \delta, \delta_{2}$ and $\zeta$ satisfying $\Delta<\delta_{1}<$ $\delta<\delta_{2}<1$ and $\zeta \in\left(0, \frac{1}{2}-\theta \alpha\right)$. Then there exist constants $c>0$ and $c_{2}>0$ such that the following conclusions hold.

(a) For all sufficiently large $n$

$$
\begin{aligned}
\left(P_{n, \beta_{n}, K_{n}} \times Q\right)\left\{A_{n}\left(\delta_{1}\right)\right\}+e^{-c n^{2 \zeta}} & \geq P_{n, \beta_{n}, K_{n}}\left\{S_{n} / n>\delta m_{n}\right\} \\
& \geq\left(P_{n, \beta_{n}, K_{n}} \times Q\right)\left\{A_{n}\left(\delta_{2}\right)\right\}-e^{-c n^{2 \zeta}} .
\end{aligned}
$$


(b) For all sufficiently large $n$

$$
\begin{aligned}
\tilde{E}_{n, \beta_{n}, K_{n}}\left\{\left|S_{n} / n^{1-\kappa}+W_{n} / n^{1 / 2-\kappa}-n^{\kappa} m_{n}\right| \cdot 1_{A_{n}\left(\delta_{1}\right)}\right\} & \\
& +2 n^{\kappa} e^{-c n^{2 \zeta}}+c_{2} n^{\kappa-1 / 2} e^{-c n^{2 \zeta} / 2} \\
\geq & \tilde{E}_{n, \beta_{n}, K_{n}}\left\{\left|S_{n} / n^{1-\kappa}+W_{n} / n^{1 / 2-\kappa}-n^{\kappa} m_{n}\right| \cdot 1_{\left\{S_{n} / n>\delta m_{n}\right\}}\right\} \\
\geq & \tilde{E}_{n, \beta_{n}, K_{n}}\left\{\left|S_{n} / n^{1-\kappa}+W_{n} / n^{1 / 2-\kappa}-n^{\kappa} m_{n}\right| \cdot 1_{A_{n}\left(\delta_{2}\right)}\right\} \\
& -2 n^{\kappa} e^{-c n^{2 \zeta}}-c_{2} n^{\kappa-1 / 2} e^{-c n^{2 \zeta} / 2} .
\end{aligned}
$$

Proof of part (a) of Lemma 8.9. We choose $\zeta \in\left(0, \frac{1}{2}-\theta \alpha\right)$. The proof is based on the following two claims, which are proved later.

Claim 1. For all sufficiently large $n,\left\{S_{n} / n>\delta m_{n}\right\} \subset A_{n}\left(\delta_{1}\right) \cup\left\{\left|W_{n}\right|>\frac{1}{2} n^{\zeta}\right\}$.

Claim 2. For all sufficiently large $n,\left\{S_{n} / n>\delta m_{n}\right\} \supset A_{n}\left(\delta_{2}\right) \backslash\left\{\left|W_{n}\right|>\frac{1}{2} n^{\zeta}\right\}$.

By Claims 1 and 2, for all sufficiently large $n$

$$
\begin{aligned}
& \left(P_{n, \beta_{n}, K_{n}} \times Q\right)\left\{A_{n}\left(\delta_{1}\right)\right\}+Q\left\{\left\{\left|W_{n}\right|>\frac{1}{2} n^{\zeta}\right\}\right. \\
& \quad=\left(P_{n, \beta_{n}, K_{n}} \times Q\right)\left\{A_{n}\left(\delta_{1}\right)\right\}+\left(P_{n, \beta_{n}, K_{n}} \times Q\right)\left\{\left\{\left|W_{n}\right|>\frac{1}{2} n^{\zeta}\right\}\right. \\
& \quad \geq\left(P_{n, \beta_{n}, K_{n}} \times Q\right)\left\{S_{n} / n>\delta m_{n}\right\}=P\left\{S_{n} / n>\delta m_{n}\right\} \\
& \quad \geq\left(P_{n, \beta_{n}, K_{n}} \times Q\right)\left\{A_{n}\left(\delta_{2}\right)\right\}-Q\left\{\left|W_{n}\right|>\frac{1}{2} n^{\zeta}\right\} .
\end{aligned}
$$

Part (a) of Lemma 8.8 completes the proof. Thus, given Claims 1 and 2, the proof of part (a) is complete.

Proof of part (b) of Lemma 8.9. We use Claim 1 to prove the first inequality in part (b). For all sufficiently large $n$ 


$$
\begin{aligned}
& \tilde{E}_{n, \beta_{n}, K_{n}}\left\{\left|S_{n} / n^{1-\kappa}+W_{n} / n^{1 / 2-\kappa}-n^{\kappa} m_{n}\right| \cdot 1_{\left\{S_{n} / n>\delta m_{n}\right\}}\right\} \\
&=\int_{\Lambda^{n} \times \Omega}\left|S_{n} / n^{1-\kappa}+W_{n} / n^{1 / 2-\kappa}-n^{\kappa} m_{n}\right| \cdot 1_{\left\{S_{n} / n>\delta m_{n}\right\}} d\left(P_{n, \beta_{n}, K_{n}} \times Q\right) \\
& \leq \int_{\Lambda^{n} \times \Omega}\left|S_{n} / n^{1-\kappa}+W_{n} / n^{1 / 2-\kappa}-n^{\kappa} m_{n}\right| \cdot 1_{A_{n}\left(\delta_{1}\right) \cup\left\{\left|W_{n}\right|>n^{\zeta}\right\}} d\left(P_{n, \beta_{n}, K_{n}} \times Q\right) \\
& \leq \int_{\Lambda^{n} \times \Omega}\left|S_{n} / n^{1-\kappa}+W_{n} / n^{1 / 2-\kappa}-n^{\kappa} m_{n}\right| \cdot 1_{A_{n}\left(\delta_{1}\right)} d\left(P_{n, \beta_{n}, K_{n}} \times Q\right) \\
&+\int_{\Lambda^{n} \times \Omega}\left|S_{n} / n^{1-\kappa}+W_{n} / n^{1 / 2-\kappa}-n^{\kappa} m_{n}\right| \cdot 1_{\left\{\left|W_{n}\right|>\frac{1}{2} n^{\zeta}\right\}} d\left(P_{n, \beta_{n}, K_{n}} \times Q\right) \\
& \leq \int_{\Lambda^{n} \times \Omega}\left|S_{n} / n^{1-\kappa}+W_{n} / n^{1 / 2-\kappa}-n^{\kappa} m_{n}\right| \cdot 1_{A_{n}\left(\delta_{1}\right)} d\left(P_{n, \beta_{n}, K_{n}} \times Q\right) \\
&+\int_{\Lambda^{n} \times \Omega}\left|S_{n} / n^{1-\kappa}-n^{\kappa} m_{n}\right| \cdot 1_{\left\{\left|W_{n}\right|>\frac{1}{2} n^{\zeta}\right\}} d\left(P_{n, \beta_{n}, K_{n}} \times Q\right) \\
&+\int_{\Omega}\left|W_{n} / n^{1 / 2-\kappa}\right| \cdot 1_{\left\{\left|W_{n}\right|>\frac{1}{2} n^{\zeta}\right\}} d Q .
\end{aligned}
$$

Since $\left|S_{n} / n\right| \leq 1$ and $m_{n} \in(0,1)$, we have $\left|S_{n} / n^{1-\kappa}-n^{\kappa} m_{n}\right| \leq 2 n^{\kappa}$. Using part (a) of Lemma 8.8, for all sufficiently large $n$ we bound the next to last integral in (8.6) by

$$
2 n^{\kappa} \cdot Q\left\{\left|W_{n}\right|>\frac{1}{2} n^{\zeta}\right\} \leq 2 n^{\kappa} \exp \left(-c n^{2 \zeta}\right)
$$

where $c>0$ is a constant. The next step is to apply the Cauchy-Schwartz inequality to the last integral in (8.6) and use parts (a) and (b) of Lemma 8.8. There exist constants $c>0$ and $c_{2}=\sqrt{c_{1}}>0$ such that for all $n$

$$
\begin{aligned}
& \int_{\Omega}\left|W_{n} / n^{1 / 2-\kappa}\right| \cdot 1_{\left\{\left|W_{n}\right|>\frac{1}{2} n^{\zeta}\right\}} d Q \\
& \quad \leq\left(\int_{\Omega}\left|W_{n} / n^{1 / 2-\kappa}\right|^{2} d Q\right)^{1 / 2} \cdot\left(Q\left\{\left|W_{n}\right|>\frac{1}{2} n^{\zeta}\right\}\right)^{1 / 2} \leq c_{2} n^{\kappa-1 / 2} \exp \left[-c n^{2 \zeta} / 2\right] .
\end{aligned}
$$

It follows that for all sufficiently large $n$

$$
\begin{aligned}
& \tilde{E}_{n, \beta_{n}, K_{n}}\left\{\left|S_{n} / n^{1-\kappa}+W_{n} / n^{1 / 2-\kappa}-n^{\kappa} m_{n}\right| \cdot 1_{\left\{S_{n} / n>\delta m_{n}\right\}}\right\} \\
& \leq \tilde{E}_{n, \beta_{n}, K_{n}}\left\{\left|S_{n} / n^{1-\kappa}+W_{n} / n^{1 / 2-\kappa}-n^{\kappa} m_{n}\right| \cdot 1_{A_{n}\left(\delta_{1}\right)}\right\} \\
& \quad+2 n^{\kappa} e^{-c n^{2 \zeta}}+c_{2} n^{\kappa-1 / 2} e^{-c n^{2 \zeta} / 2} .
\end{aligned}
$$


This completes the proof of the first inequality in part (b).

We now use Claim 2 to prove the second inequality in part (b). For all sufficiently large n

$$
\begin{aligned}
& \tilde{E}_{n, \beta_{n}, K_{n}}\left\{\left|S_{n} / n^{1-\kappa}+W_{n} / n^{1 / 2-\kappa}-n^{\kappa} m_{n}\right| \cdot 1_{\left\{S_{n} / n>\delta m_{n}\right\}}\right\} \\
&=\int_{\Lambda^{n} \times \Omega}\left|S_{n} / n^{1-\kappa}+W_{n} / n^{1 / 2-\kappa}-n^{\kappa} m_{n}\right| \cdot 1_{\left\{S_{n} / n>\delta m_{n}\right\}} d P_{n, \beta_{n}, K_{n}} \\
& \geq \int_{\Lambda^{n} \times \Omega}\left|S_{n} / n^{1-\kappa}+W_{n} / n^{1 / 2-\kappa}-n^{\kappa} m_{n}\right| \cdot 1_{A_{n}\left(\delta_{2}\right) \backslash\left\{\left|W_{n}\right|>\frac{1}{2} n^{\zeta}\right\}} d\left(P_{n, \beta_{n}, K_{n}} \times Q\right) \\
& \geq \int_{\Lambda^{n} \times \Omega}\left|S_{n} / n^{1-\kappa}+W_{n} / n^{1 / 2-\kappa}-n^{\kappa} m_{n}\right| \cdot 1_{A_{n}\left(\delta_{2}\right)} d\left(P_{n, \beta_{n}, K_{n}} \times Q\right) \\
&-\int_{\Lambda^{n} \times \Omega}\left|S_{n} / n^{1-\kappa}+W_{n} / n^{1 / 2-\kappa}-n^{\kappa} m_{n}\right| \cdot 1_{\left\{\left|W_{n}\right|>\frac{1}{2} n^{\zeta}\right\}} d\left(P_{n, \beta_{n}, K_{n}} \times Q\right) \\
& \geq \int_{\Lambda^{n} \times \Omega}\left|S_{n} / n^{1-\kappa}+W_{n} / n^{1 / 2-\kappa}-n^{\kappa} m_{n}\right| \cdot 1_{A_{n}\left(\delta_{2}\right)} d\left(P_{n, \beta_{n}, K_{n}} \times Q\right) \\
&-\int_{\Lambda^{n} \times \Omega}\left|S_{n} / n^{1-\kappa}-n^{\kappa} m_{n}\right| \cdot 1_{\left\{\left|W_{n}\right|>\frac{1}{2} n^{\zeta}\right\}} d\left(P_{n, \beta_{n}, K_{n}} \times Q\right) \\
&-\int_{\Omega}\left|W_{n} / n^{1 / 2-\kappa}\right| \cdot 1_{\left\{\left|W_{n}\right|>\frac{1}{2} n^{\zeta}\right\}} d Q .
\end{aligned}
$$

The next to last inequality follows from the fact that for any sets $C$ and $D, 1_{C \backslash D} \geq 1_{C}-1_{D}$. The last two integrals in (8.8) coincide with the last two integrals in (8.6) and hence can be bounded the same way. For all sufficiently large $n$ this yields

$$
\begin{aligned}
& \tilde{E}_{n, \beta_{n}, K_{n}}\left\{\left|S_{n} / n^{1-\kappa}+W_{n} / n^{1 / 2-\kappa}-n^{\kappa} m_{n}\right| \cdot 1_{\left\{S_{n} / n>\delta m_{n}\right\}}\right\} \\
& \geq \tilde{E}_{n, \beta_{n}, K_{n}}\left\{\left|S_{n} / n^{1-\kappa}+W_{n} / n^{1 / 2-\kappa}-n^{\kappa} m_{n}\right| \cdot 1_{A_{n}\left(\delta_{2}\right)}\right\} \\
& \quad-2 n^{\kappa} e^{-c n^{2 \zeta}}-c_{2} n^{\kappa-1 / 2} e^{-c n^{2 \zeta / 2}}
\end{aligned}
$$

where $c>0$ and $c_{2}>0$ are constants. In combination with (8.7), the last inequality yields part (b).

In order to complete the proofs of parts (a) and (b) we now turn to the proofs of Claims 1 and 2. 
Proof of Claim 1. We write

$$
\begin{aligned}
\left\{S_{n} / n>\delta m_{n}\right\} & =\left(\left\{S_{n} / n>\delta m_{n}\right\} \cap\left\{\left|W_{n}\right| \leq \frac{1}{2} n^{\zeta}\right\}\right) \cup\left(\left\{S_{n} / n>\delta m_{n}\right\} \cap\left\{\left|W_{n}\right|>\frac{1}{2} n^{\zeta}\right\}\right) \\
& \subset\left(\left\{S_{n} / n>\delta m_{n}\right\} \cap\left\{\left|W_{n}\right| \leq \frac{1}{2} n^{\zeta}\right\}\right) \cup\left\{\left|W_{n}\right|>\frac{1}{2} n^{\zeta}\right\} .
\end{aligned}
$$

Claim 1 follows if we prove for all sufficiently large $n$

$$
\left\{S_{n} / n>\delta m_{n}\right\} \cap\left\{\left|W_{n}\right| \leq \frac{1}{2} n^{\zeta}\right\} \subset A_{n}\left(\delta_{1}\right)=\left\{S_{n} / n+W_{n} / n^{1 / 2}>\delta_{1} m_{n}\right\}
$$

We have

$$
\begin{aligned}
\left\{S_{n} / n>\delta m_{n}\right\} \cap\left\{\left|W_{n}\right| \leq \frac{1}{2} n^{\zeta}\right\}= & \left(\left\{S_{n} / n>\delta m_{n}\right\} \cap\left\{0 \leq W_{n} \leq \frac{1}{2} n^{\zeta}\right\}\right) \\
& \cup\left(\left\{S_{n} / n>\delta m_{n}\right\} \cap\left\{-\frac{1}{2} n^{\zeta} \leq W_{n}<0\right\}\right)
\end{aligned}
$$

If $S_{n} / n>\delta m_{n}$ and $0 \leq W_{n} \leq \frac{1}{2} n^{\zeta}$, then $S_{n} / n+W_{n} / n^{1 / 2} \geq S_{n} / n>\delta m_{n}>\delta_{1} m_{n}$. Thus

$$
\left\{S_{n} / n>\delta m_{n}\right\} \cap\left\{0 \leq W_{n} \leq \frac{1}{2} n^{\zeta}\right\} \subset A_{n}\left(\delta_{1}\right)
$$

Now assume that $S_{n} / n>\delta m_{n}$ and $-\frac{1}{2} n^{\zeta} \leq W_{n}<0$. Since $\zeta<\frac{1}{2}-\theta \alpha$, we have for all sufficiently large $n$

$$
\left(\delta-\delta_{1}\right) \bar{x}>n^{\zeta-1 / 2+\theta \alpha} .
$$

Since $\lim _{n \rightarrow \infty} n^{\theta \alpha} m_{n}=\bar{x}$ [Thm. 3.1], it follows that for all sufficiently large $n$

$$
\left(\delta-\delta_{1}\right) m_{n}>\frac{1}{2} n^{\zeta-1 / 2}
$$

Thus $\delta m_{n}-\frac{1}{2} n^{\zeta-1 / 2}>\delta_{1} m_{n}$ for all sufficiently large $n$. Hence, if $S_{n} / n>\delta m_{n}$ and $W_{n} / n^{1 / 2} \geq-\frac{1}{2} n^{\zeta-1 / 2}$, then for all sufficiently large $n$

$$
S_{n} / n+W_{n} / n^{1 / 2}>\delta m_{n}+W_{n} / n^{1 / 2} \geq \delta m_{n}-\frac{1}{2} n^{\zeta-1 / 2}>\delta_{1} m_{n}
$$


It follows that for all sufficiently large $n$

$$
\left\{S_{n} / n>\delta m_{n}\right\} \cap\left\{-\frac{1}{2} n^{\zeta} \leq W_{n}<0\right\} \subset A_{n}\left(\delta_{1}\right)
$$

Therefore (8.10) follows from (8.11) and the last display. This completes the proof of Claim 1.

Proof of Claim 2. It suffices to prove that $A_{n}\left(\delta_{2}\right) \subset\left\{S_{n} / n>\delta m_{n}\right\} \cup\left\{\left|W_{n}\right|>\frac{1}{2} n^{\zeta}\right\}$. We write

$$
\begin{aligned}
A_{n}\left(\delta_{2}\right)= & \left\{S_{n} / n+W_{n} / n^{1 / 2}>\delta_{2} m_{n}\right\} \\
= & \left(\left\{S_{n} / n+W_{n} / n^{1 / 2}>\delta_{2} m_{n}\right\} \cap\left\{\left|W_{n}\right|>\frac{1}{2} n^{\zeta}\right\}\right) \\
& \cup\left(\left\{S_{n} / n+W_{n} / n^{1 / 2}>\delta_{2} m_{n}\right\} \cap\left\{\left|W_{n}\right| \leq \frac{1}{2} n^{\zeta}\right\}\right) . \\
\subset \quad & \left\{\left|W_{n}\right|>\frac{1}{2} n^{\zeta}\right\} \cup\left(\left\{S_{n} / n+W_{n} / n^{1 / 2}>\delta_{2} m_{n}\right\} \cap\left\{\left|W_{n}\right| \leq \frac{1}{2} n^{\zeta}\right\} .\right.
\end{aligned}
$$

Hence Claim 2 follows if we prove for all sufficiently large $n$

$$
\left\{S_{n} / n+W_{n} / n^{1 / 2}>\delta_{2} m_{n}\right\} \cap\left\{\left|W_{n}\right| \leq \frac{1}{2} n^{\zeta}\right\} \subset\left\{S_{n} / n>\delta m_{n}\right\}
$$

We have

$$
\begin{aligned}
& \left\{S_{n} / n+W_{n} / n^{1 / 2}>\delta_{2} m_{n}\right\} \cap\left\{\left|W_{n}\right| \leq \frac{1}{2} n^{\zeta}\right\} \\
& =\left(\left\{S_{n} / n+W_{n} / n^{1 / 2}>\delta_{2} m_{n}\right\} \cap\left\{0<W_{n} \leq \frac{1}{2} n^{\zeta}\right\}\right) \\
& \cup\left(\left\{S_{n} / n+W_{n} / n^{1 / 2}>\delta_{2} m_{n}\right\} \cap\left\{-\frac{1}{2} n^{\zeta} \leq W_{n} \leq 0\right\}\right) .
\end{aligned}
$$

If $S_{n} / n+W_{n} / n^{1 / 2}>\delta_{2} m_{n}$ and $-\frac{1}{2} n^{\zeta} \leq W_{n} \leq 0$, then $S_{n} / n \geq S_{n} / n+W_{n} / n^{1 / 2}>$ $\delta_{2} m_{n}>\delta m_{n}$. Thus

$$
\left\{S_{n} / n+W_{n} / n^{1 / 2}>\delta_{2} m_{n}\right\} \cap\left\{-\frac{1}{2} n^{\zeta} \leq W_{n} \leq 0\right\} \subset\left\{S_{n} / n>\delta m_{n}\right\}
$$


Now assume that $S_{n} / n+W_{n} / n^{1 / 2}>\delta_{2} m_{n}$ and $0<W_{n} \leq \frac{1}{2} n^{\zeta}$. Since $\zeta<\frac{1}{2}-\theta \alpha$, we have for all sufficiently large $n$

$$
\left(\delta_{2}-\delta\right) \bar{x}>n^{\zeta-1 / 2+\theta \alpha}
$$

Since $\lim _{n \rightarrow \infty} n^{\theta \alpha} m_{n}=\bar{x}$ [Thm. 3.1], we have for all sufficiently large $n$

$$
\left(\delta_{2}-\delta\right) m_{n}>\frac{1}{2} n^{\zeta-1 / 2}
$$

Thus $\delta_{2} m_{n}-\frac{1}{2} n^{\zeta-1 / 2}>\delta m_{n}$ for all sufficiently large $n$. Hence, if $S_{n} / n+W_{n} / n^{1 / 2}>\delta_{2} m_{n}$ and $0<W_{n} / n^{1 / 2} \leq \frac{1}{2} n^{\zeta-1 / 2}$, then for all sufficiently large $n$

$$
S_{n} / n>\delta_{2} m_{n}-W_{n} / n^{1 / 2} \geq \delta_{2} m_{n}-\frac{1}{2} n^{\zeta-1 / 2}>\delta m_{n}
$$

Thus for all sufficiently large $n$

$$
\left\{S_{n} / n+W_{n} / n^{1 / 2}>\delta_{2} m_{n}\right\} \cap\left\{0<W_{n} \leq \frac{1}{2} n^{\zeta}\right\} \subset\left\{S_{n} / n>\delta m_{n}\right\}
$$

Therefore (8.12) follows from (8.13) and (8.14). This completes the proof of Claim 2. The proof of Lemma 8.9 is complete.

We now prove Lemma 8.5, completing the preparatory lemmas that will be used in the next section to prove part (b) of Theorem 6.1.

Proof of Lemma 8.5. This is done first when $g$ has degree 4 and second when $g$ has degree 6. 
Proof of part (a) of Lemma 8.5 when $g$ has degree 4. By Taylor's theorem, for any $R>0$, all $n \in \mathbb{N}$, and all $x \in \mathbb{R}$ satisfying $\left|x / n^{\kappa}\right|<R$

$$
\begin{aligned}
& n G_{\beta_{n}, K_{n}}\left(x / n^{\kappa}+m_{n}\right)-n G_{\beta_{n}, K_{n}}\left(m_{n}\right) \\
& \quad=\sum_{j=1}^{4} \frac{1}{n^{j \kappa-1}} \cdot \frac{G_{\beta_{n}, K_{n}}^{(j)}\left(m_{n}\right)}{j !} x^{j}+\frac{1}{n^{5 \kappa-1}} \cdot \frac{G_{\beta_{n}, K_{n}}^{(5)}\left(m_{n}+\tau x / n^{\kappa}\right)}{5 !} x^{5},
\end{aligned}
$$

where $\tau$ is a number in $[0,1]$. The quantity $m_{n}+\tau x / n^{\kappa}$ lies in the interval $\left[m_{n}-\right.$ $\left.\left|x / n^{\kappa}\right|, m_{n}+\left|x / n^{\kappa}\right|\right]$. Since $m_{n} \in(0,1), m_{n} \rightarrow 0$ and $\left|x / n^{\kappa}\right|<R$, we have $m_{n}+\tau x / n^{\kappa} \in$ $(-R, R+1)$ for all $n$. Since the sequence $\left(\beta_{n}, K_{n}\right)$ is bounded and positive, there exists $a \in(0, \infty)$ such that $0 \leq \beta_{n} \leq a$ and $0 \leq K_{n} \leq a$ for all $n$. As a continuous function of $(\beta, K, y)$ on the compact set $[0, a] \times[0, a] \times[-R, R+1]$, it follows that $G_{\beta, K}^{(5)}(y)$ is uniformly bounded. Since $m_{n}+\tau x / n^{\kappa} \in(-R, R+1)$ for all $n \in \mathbb{N}$ and $x \in \mathbb{R}$ satisfying $\left|x / n^{\kappa}\right|<R, G_{\beta_{n}, K_{n}}^{(5)}\left(m_{n}+\tau x / n^{\kappa}\right)$ is uniformly bounded for $n \in \mathbb{N}$ and $x \in\left(-R n^{\kappa}, R n^{\kappa}\right)$.

We summarize the last display by writing

$$
\begin{aligned}
& n G_{\beta_{n}, K_{n}}\left(x / n^{\kappa}+m_{n}\right)-n G_{\beta_{n}, K_{n}}\left(m_{n}\right) \\
& \quad=\sum_{j=1}^{4} \frac{1}{n^{j \kappa-1}} \cdot \frac{G_{\beta_{n}, K_{n}}^{(j)}\left(m_{n}\right)}{j !} x^{j}+\mathrm{O}\left(\frac{1}{n^{5 \kappa-1}}\right) x^{5},
\end{aligned}
$$

where the big-oh term is uniform for $x \in\left(-R n^{\kappa}, R n^{\kappa}\right)$.

Let $\varepsilon_{n}$ denote a sequence that converges to 0 and that represents the various error terms arising in the proof. The same notation $\varepsilon_{n}$ will be used to represent different error terms. To simplify the arithmetic, we introduce $u=1-\alpha / \alpha_{0}>0$. We have the following three properties:

(1) Since $m_{n}$ is the unique positive, global minimum point of $G_{\beta_{n}, K_{n}}, G_{\beta_{n}, K_{n}}^{(1)}\left(m_{n}\right)=0$.

(2) By hypothesis (iii') of Theorem 4.1, for $j=2,3,4$, we have

$$
G_{\beta_{n}, K_{n}}^{(j)}\left(m_{n}\right)=\left(g^{(j)}(\bar{x})+\varepsilon_{n}\right) / n^{\alpha / \alpha_{0}-j \theta \alpha}=\left(g^{(j)}(\bar{x})+\varepsilon_{n}\right) / n^{1-u-j \theta \alpha} .
$$


(3) Since $\kappa=\frac{1}{2} u+\theta \alpha$, we have $j \kappa-u-j \theta \alpha=\left(\frac{j}{2}-1\right) u$ for $j=2,3,4$.

Using these properties, we obtain the following asymptotic formula, which is valid for any $R>0$, all $n \in \mathbb{N}$, and all $x \in \mathbb{R}$ satisfying $\left|x / n^{\kappa}\right|<R$ :

$$
\begin{aligned}
n G_{\beta_{n}, K_{n}}\left(x / n^{\kappa}+m_{n}\right)-n G_{\beta_{n}, K_{n}}\left(m_{n}\right)= & \sum_{j=2}^{4} \frac{1}{n^{j \kappa-1}} \cdot \frac{g^{(j)}(\bar{x})+\varepsilon_{n}}{n^{1-u-j \theta \alpha} \cdot j !} \cdot x^{j}+\mathrm{O}\left(\frac{1}{n^{5 \kappa-1}}\right) x^{5} \\
= & \sum_{j=2}^{4} \frac{1}{j !} \cdot \frac{g^{(j)}(\bar{x})+\varepsilon_{n}}{n^{(j / 2-1) u}} \cdot x^{j}+\mathrm{O}\left(\frac{1}{n^{5 \kappa-1}}\right) x^{5} \\
= & \frac{1}{2 !} \cdot\left(g^{(2)}(\bar{x})+\varepsilon_{n}\right) x^{2}+\frac{1}{3 !} \cdot \frac{\left(g^{(3)}(\bar{x})+\varepsilon_{n}\right)}{n^{u / 2}} x^{3} \\
& +\frac{1}{4 !} \cdot \frac{\left(g^{(4)}(\bar{x})+\varepsilon_{n}\right)}{n^{u}} x^{4}+\mathrm{O}\left(\frac{1}{n^{5 \kappa-1}}\right) x^{5} .
\end{aligned}
$$

By hypothesis (iii') of Theorem 4.1 and part (c) of Theorem 6.1, we have $1 / 4 \leq \theta \alpha_{0}<$ $\kappa<1 / 2$. Therefore $5 \kappa-1>5 \theta \alpha_{0}-1>0$. Since $u>0$ and $\varepsilon_{n} \rightarrow 0$, we have for all $x \in \mathbb{R}$

$$
\lim _{n \rightarrow \infty}\left(n G_{\beta_{n}, K_{n}}\left(x / n^{\kappa}+m_{n}\right)-n G_{\beta_{n}, K_{n}}\left(m_{n}\right)\right)=\frac{1}{2} g^{(2)}(\bar{x}) x^{2} .
$$

This completes the proof of part (a) of Lemma 8.5 when $g$ has degree 4 .

Proof of part (b) of Lemma 8.5 when $g$ has degree 4. Hypothesis (iii') of Theorem 4.1 states that $g^{(j)}(\bar{x})>0$ for $j=2,3,4$. It follows that for all sufficiently large $n$ and all $x \in \mathbb{R}$

$$
\frac{1}{2 !} \cdot\left(g^{(2)}(\bar{x})+\varepsilon_{n}\right) x^{2} \geq \frac{1}{2 \cdot 2 !} \cdot g^{(2)}(\bar{x}) x^{2}
$$

and

$$
\frac{1}{4 !} \cdot \frac{\left(g^{(4)}(\bar{x})+\varepsilon_{n}\right)}{n^{u}} x^{4} \geq \frac{1}{2 \cdot 4 !} \cdot \frac{g^{(4)}(\bar{x})}{n^{u}} x^{4}
$$

and that for all sufficiently large $n$

$$
\frac{1}{3 !} \cdot \frac{g^{(3)}(\bar{x})+\varepsilon_{n}}{n^{u / 2}} x^{3} \geq \frac{1}{2 \cdot 3 !} \cdot \frac{g^{(3)}(\bar{x})}{n^{u / 2}} x^{3} \text { for all } x \geq 0
$$


and

$$
\frac{1}{3 !} \cdot \frac{g^{(3)}(\bar{x})+\varepsilon_{n}}{n^{u / 2}} x^{3} \geq \frac{2}{3 !} \cdot \frac{g^{(3)}(\bar{x})}{n^{u / 2}} x^{3} \text { for all } x<0 .
$$

We first consider $x \in\left[0, R n^{\kappa}\right)$. Since $g^{(4)}(\bar{x})>0$, for all sufficiently large $n$ and all such $x$ we have

$$
\begin{aligned}
n G_{\beta_{n}, K_{n}}\left(x / n^{\kappa}+m_{n}\right)-n G_{\beta_{n}, K_{n}}\left(m_{n}\right) \\
\geq \quad \frac{1}{2 \cdot 2 !} \cdot g^{(2)}(\bar{x}) x^{2}+\frac{1}{2 \cdot 3 !} \cdot \frac{g^{(3)}(\bar{x})}{n^{u / 2}} x^{3} \\
\quad+\frac{1}{2 \cdot 4 !} \cdot \frac{g^{(4)}(\bar{x})}{n^{u}} x^{4}+\frac{1}{n^{u}} \mathrm{O}\left(\frac{x}{n^{5 \kappa-1-u}}\right) x^{4} \\
\geq \quad \frac{1}{2 \cdot 2 !} \cdot g^{(2)}(\bar{x}) x^{2}+\frac{1}{2 \cdot 3 !} \cdot \frac{g^{(3)}(\bar{x})}{n^{u / 2}} x^{3} \\
\quad+\frac{1}{2 \cdot 4 !} \cdot \frac{g^{(4)}(\bar{x})}{n^{u}} x^{4}\left(1+\mathrm{O}\left(x / n^{5 \kappa-1-u}\right)\right) .
\end{aligned}
$$

By hypothesis (iii') of Theorem 4.1, $\theta \alpha_{0} \in[1 / 4,1 / 2)$. Hence $4 \theta-1 / \alpha_{0} \geq 0$, and so

$$
5 \kappa-1-u=\kappa+\left(4 \theta-1 / \alpha_{0}\right) \alpha \geq \kappa .
$$

Hence for all $0<x<R n^{\kappa}$ we have $0 \leq x / n^{5 \kappa-1-u} \leq x / n^{\kappa}<R$. Thus the term $\mathrm{O}\left(x / n^{5 \kappa-1-u}\right)$ appearing in (8.15) can be made larger than -1 for all $0 \leq x / n^{\kappa}<$ $R$ by choosing $R$ to be sufficiently small. Since $g^{(3)}(\bar{x})>0, g^{(4)}(\bar{x})>0$, and $1+$ $\mathrm{O}\left(x / n^{5 \kappa-1-u}\right)>0$, we have that for all sufficiently large $n$ and all $x \in\left[0, R n^{\kappa}\right)$

$$
n G_{\beta_{n}, K_{n}}\left(x / n^{\kappa}+m_{n}\right)-n G_{\beta_{n}, K_{n}}\left(m_{n}\right) \geq \frac{1}{2 \cdot 2 !} g^{(2)}(\bar{x}) x^{2} \geq \frac{1}{8} g^{(2)}(\bar{x}) x^{2} .
$$

This is the conclusion of part (b) of Lemma 8.5 for all $0 \leq x<R n^{\kappa}$ when $g$ has degree 4 . 
We now consider $x \in\left(-R n^{\kappa}, 0\right]$. Since $g^{(4)}(\bar{x})>0$, for all sufficiently large $n$ and all such $x$ we have

$$
\begin{aligned}
n G_{\beta_{n}, K_{n}}\left(x / n^{\kappa}+m_{n}\right)-n G_{\beta_{n}, K_{n}}\left(m_{n}\right) \geq & \frac{1}{2 \cdot 2 !} \cdot g^{(2)}(\bar{x}) x^{2}+\frac{2}{3 !} \cdot \frac{g^{(3)}(\bar{x})}{n^{u / 2}} x^{3} \\
& +\frac{1}{2 \cdot 4 !} \cdot \frac{g^{(4)}(\bar{x})}{n^{u}} x^{4}+\frac{1}{n^{u}} \mathrm{O}\left(\frac{x}{n^{5 \kappa-1-u}}\right) x^{4} \\
\geq & \frac{1}{2 \cdot 2 !} \cdot g^{(2)}(\bar{x}) x^{2}+\frac{2}{3 !} \cdot \frac{g^{(3)}(\bar{x})}{n^{u / 2}} x^{3} \\
& +\frac{1}{2 \cdot 4 !} \cdot \frac{g^{(4)}(\bar{x})}{n^{u}} x^{4}\left(1+\mathrm{O}\left(x / n^{5 \kappa-1-u}\right)\right) .
\end{aligned}
$$

Since $5 \kappa-1-u \geq \kappa$, for all $-R n^{\kappa}<x<0$ we have $-R<x / n^{\kappa} \leq x / n^{5 \kappa-1-u}<$ 0 . Thus the term $\mathrm{O}\left(x / n^{5 \kappa-1-u}\right)$ appearing in (8.16) can be made larger than -1 for all $-R<x / n^{\kappa}<0$ by choosing $R$ to be sufficiently small. Since $g^{(4)}(\bar{x})>0$ and $1+$ $\mathrm{O}\left(x / n^{5 \kappa-1-u}\right)>0$, we have that for all sufficiently large $n$ and all $x \in\left(-R n^{\kappa}, 0\right)$

$$
n G_{\beta_{n}, K_{n}}\left(x / n^{\kappa}+m_{n}\right)-n G_{\beta_{n}, K_{n}}\left(m_{n}\right) \geq \frac{1}{2 \cdot 2 !} g^{(2)}(\bar{x}) x^{2}+\frac{2}{3 !} \cdot \frac{g^{(3)}(\bar{x})}{n^{u / 2}} x^{3} .
$$

By Theorem 3.1 we have $m_{n} \sim \bar{x} / n^{\theta \alpha}$. Thus $n^{\theta \alpha} m_{n}=\bar{x}+\varepsilon_{n}$, and

$$
n^{\kappa} m_{n}=n^{u / 2} \cdot n^{\theta \alpha} m_{n}=n^{u / 2}\left(\bar{x}+\varepsilon_{n}\right)
$$

In part (b) of Lemma 8.5 we assume that $x / n^{\kappa}>-(1-\bar{\delta}) m_{n}$ and $0<\bar{\delta}<1$. Thus for all sufficiently large $n$ and all such $x$

$$
x>-(1-\bar{\delta}) n^{\kappa} m_{n}=-(1-\bar{\delta}) n^{u / 2}\left(\bar{x}+\varepsilon_{n}\right) \geq-(1-\bar{\delta}) n^{u / 2} \cdot 2 \bar{x} .
$$

Since $g^{(3)}(\bar{x})>0$, we see that for all sufficiently large $n$, all $x \in\left(-R n^{\kappa}, 0\right)$, and all $x / n^{\kappa}>-(1-\bar{\delta}) m_{n}$ there exists $\Delta \in(0,1)$ such that for any $\bar{\delta} \in(\Delta, 1)$ the following inequalities hold: 


$$
\begin{aligned}
n & G_{\beta_{n}, K_{n}}\left(x / n^{\kappa}+m_{n}\right)-n G_{\beta_{n}, K_{n}}\left(m_{n}\right) \\
& \geq \frac{1}{2 \cdot 2 !} \cdot g^{(2)}(\bar{x}) x^{2}+\frac{2}{3 !} \cdot \frac{g^{(3)}(\bar{x})}{n^{u / 2}} x^{2} \cdot\left[-(1-\bar{\delta}) n^{u / 2} \cdot 2 \bar{x}\right] \\
& =\left(\frac{1}{2 \cdot 2 !} \cdot g^{(2)}(\bar{x})-\frac{2 \cdot 2}{3 !} \cdot g^{(3)}(\bar{x})(1-\bar{\delta}) \bar{x}\right) x^{2} \\
& \geq \frac{1}{2} \cdot \frac{1}{2 \cdot 2 !} g^{(2)}(\bar{x}) x^{2}=\frac{1}{8} g^{(2)}(\bar{x}) x^{2} .
\end{aligned}
$$

This is the conclusion of part (b) of Lemma 8.5 for $-R n^{\kappa}<x<0$ and $x / n^{\kappa}>-(1-\bar{\delta}) m_{n}$ when $g$ has degree 4 .

We have shown that for any $\bar{\delta} \in(\Delta, 1)$ there exists $R>0$ such that for all sufficiently large $n$ and all $x \in \mathbb{R}$ satisfying $\left|x / n^{\kappa}\right|<R$ and $x / n^{\kappa}>-(1-\bar{\delta}) m_{n}$

$$
n G_{\beta_{n}, K_{n}}\left(x / n^{\kappa}+m_{n}\right)-n G_{\beta_{n}, K_{n}}\left(m_{n}\right) \geq \frac{1}{8} g^{(2)}(\bar{x}) x^{2}
$$

This completes the proof of part (b) of Lemma 8.5 when $g$ has degree 4 .

We now prove Lemma 8.5 when $g$ has degree 6 .

Proof of part (a) of Lemma 8.5 when $\boldsymbol{g}$ has degree 6. We give the details of the proof, which follows the same pattern as when $g$ has degree 4. By Taylor's theorem, for any $R>0$, all $n \in \mathbb{N}$, and all $x \in \mathbb{R}$ satisfying $\left|x / n^{\kappa}\right|<R$

$$
\begin{aligned}
& n G_{\beta_{n}, K_{n}}\left(x / n^{\kappa}+m_{n}\right)-n G_{\beta_{n}, K_{n}}\left(m_{n}\right) \\
& \quad=\sum_{j=1}^{6} \frac{1}{n^{j \kappa-1}} \cdot \frac{G_{\beta_{n}, K_{n}}^{(j)}\left(m_{n}\right)}{j !} x^{j}+\frac{1}{n^{7 \kappa-1}} \cdot \frac{G_{\beta_{n}, K_{n}}^{(7)}\left(m_{n}+\tau x / n^{\kappa}\right)}{7 !} x^{7},
\end{aligned}
$$

where $\tau$ is a number in $[0,1]$. By the same argument as when $g$ has degree $4, G_{\beta_{n}, K_{n}}^{(7)}\left(m_{n}+\right.$ $\left.\tau x / n^{\kappa}\right)$ is uniformly bounded for $n \in \mathbb{N}$ and $x \in\left(-R n^{\kappa}, R n^{\kappa}\right)$. We summarize the last display by writing 


$$
\begin{aligned}
& n G_{\beta_{n}, K_{n}}\left(x / n^{\kappa}+m_{n}\right)-n G_{\beta_{n}, K_{n}}\left(m_{n}\right) \\
& \quad=\sum_{j=1}^{6} \frac{1}{n^{j \kappa-1}} \cdot \frac{G_{\beta_{n}, K_{n}}^{(j)}\left(m_{n}\right)}{j !} x^{j}+\mathrm{O}\left(\frac{1}{n^{7 \kappa-1}}\right) x^{7},
\end{aligned}
$$

where the big-oh term is uniform for $x \in\left(-R n^{\kappa}, R n^{\kappa}\right)$.

Let $\varepsilon_{n}$ denote a sequence that converges to 0 and that represents the various error terms arising in the proof. The same notation $\varepsilon_{n}$ will be used to represent different error terms. To simplify the arithmetic, we introduce $u=1-\alpha / \alpha_{0}>0$. We have the following three properties:

(1) Since $m_{n}$ is the unique global minimum point of $G_{\beta_{n}, K_{n}}, G_{\beta_{n}, K_{n}}^{(1)}\left(m_{n}\right)=0$.

(2) By hypothesis (iii') of Theorem 4.1, for $j=2,3,4,5,6$, we have

$$
G_{\beta_{n}, K_{n}}^{(j)}\left(m_{n}\right)=\left(g^{(j)}(\bar{x})+\varepsilon_{n}\right) / n^{\alpha / \alpha_{0}-j \theta \alpha}=\left(g^{(j)}(\bar{x})+\varepsilon_{n}\right) / n^{1-u-j \theta \alpha} .
$$

(3) Since $\kappa=\frac{1}{2} u+\theta \alpha$, we have $j \kappa-u-j \theta \alpha=\left(\frac{j}{2}-1\right) u$ for $j=2,3,4,5,6$.

Using these properties, we obtain the following asymptotic formula, which is valid for any $R>0$, all $n \in \mathbb{N}$, and all $x \in \mathbb{R}$ satisfying $\left|x / n^{\kappa}\right|<R$ :

$$
\begin{aligned}
n G_{\beta_{n}, K_{n}}\left(x / n^{\kappa}+m_{n}\right)-n G_{\beta_{n}, K_{n}}\left(m_{n}\right)= & \sum_{j=2}^{6} \frac{1}{n^{j \kappa-1}} \cdot \frac{g^{(j)}(\bar{x})+\varepsilon_{n}}{n^{1-u-j \theta \alpha} \cdot j !} \cdot x^{j}+\mathrm{O}\left(\frac{1}{n^{7 \kappa-1}}\right) x^{7} \\
= & \sum_{j=2}^{6} \frac{1}{j !} \cdot \frac{g^{(j)}(\bar{x})+\varepsilon_{n}}{n^{(j / 2-1) u}} \cdot x^{j}+\mathrm{O}\left(\frac{1}{n^{7 \kappa-1}}\right) x^{7} \\
= & \frac{1}{2 !} \cdot\left(g^{(2)}(\bar{x})+\varepsilon_{n}\right) x^{2}+\frac{1}{3 !} \cdot \frac{\left(g^{(3)}(\bar{x})+\varepsilon_{n}\right)}{n^{u / 2}} x^{3} \\
& +\frac{1}{4 !} \cdot \frac{\left(g^{(4)}(\bar{x})+\varepsilon_{n}\right)}{n^{u}} x^{4}+\frac{1}{5 !} \cdot \frac{\left(g^{(5)}(\bar{x})+\varepsilon_{n}\right)}{n^{3 u / 2}} x^{5} \\
& +\frac{1}{6 !} \cdot \frac{\left(g^{(6)}(\bar{x})+\varepsilon_{n}\right)}{n^{2 u}} x^{6}+\mathrm{O}\left(\frac{1}{n^{7 \kappa-1}}\right) x^{7} .
\end{aligned}
$$


By hypothesis (iii') of Theorem 4.1 and part (c) of Theorem 6.1, we have $1 / 6 \leq \theta \alpha_{0}<$ $\kappa<1 / 2$. Therefore $7 \kappa-1>7 \theta \alpha_{0}-1>0$. Since $u>0$ and $\varepsilon_{n} \rightarrow 0$, we have for all $x \in \mathbb{R}$

$$
\lim _{n \rightarrow \infty} n G_{\beta_{n}, K_{n}}\left(x / n^{\kappa}+m_{n}\right)-n G_{\beta_{n}, K_{n}}\left(m_{n}\right)=\frac{1}{2} g^{(2)}(\bar{x}) x^{2} .
$$

This completes the proof of part (a) of Lemma 8.5 when $g$ has degree 6 .

Proof of part (b) of Lemma 8.5 when $\boldsymbol{g}$ has degree 6. Hypothesis (iii') of Theorem 4.1 states that $g^{(j)}(\bar{x})>0$ for $j=2,3,4,5,6$. It follows that for all sufficiently large $n$ and all $x \in \mathbb{R}$

$$
\begin{aligned}
& \frac{1}{2 !} \cdot\left(g^{(2)}(\bar{x})+\varepsilon_{n}\right) x^{2} \geq \frac{1}{2 \cdot 2 !} \cdot g^{(2)}(\bar{x}) x^{2}, \\
& \frac{1}{4 !} \cdot \frac{\left(g^{(4)}(\bar{x})+\varepsilon_{n}\right)}{n^{u}} x^{4} \geq \frac{1}{2 \cdot 4 !} \cdot \frac{g^{(4)}(\bar{x})}{n^{u}} x^{4},
\end{aligned}
$$

and

$$
\frac{1}{6 !} \cdot \frac{\left(g^{(6)}(\bar{x})+\varepsilon_{n}\right)}{n^{2 u}} x^{6} \geq \frac{1}{2 \cdot 6 !} \cdot \frac{g^{(6)}(\bar{x})}{n^{u}} x^{6}
$$

and that for all sufficiently large $n$

$$
\begin{gathered}
\frac{1}{3 !} \cdot \frac{g^{(3)}(\bar{x})+\varepsilon_{n}}{n^{u / 2}} x^{3} \geq \frac{1}{2 \cdot 3 !} \cdot \frac{g^{(3)}(\bar{x})}{n^{u / 2}} x^{3} \text { for all } x>0, \\
\frac{1}{5 !} \cdot \frac{g^{(5)}(\bar{x})+\varepsilon_{n}}{n^{3 u / 2}} x^{5} \geq \frac{1}{2 \cdot 5 !} \cdot \frac{g^{(5)}(\bar{x})}{n^{3 u / 2}} x^{5} \text { for all } x>0, \\
\frac{1}{3 !} \cdot \frac{g^{(3)}(\bar{x})+\varepsilon_{n}}{n^{u / 2}} x^{3} \geq \frac{2}{3 !} \cdot \frac{g^{(3)}(\bar{x})}{n^{u / 2}} x^{3} \text { for all } x<0,
\end{gathered}
$$

and

$$
\frac{1}{5 !} \cdot \frac{g^{(5)}(\bar{x})+\varepsilon_{n}}{n^{3 u / 2}} x^{5} \geq \frac{2}{5 !} \cdot \frac{g^{(5)}(\bar{x})}{n^{3 u / 2}} x^{5} \text { for all } x<0 \text {. }
$$


We first consider $x \in\left[0, R n^{\kappa}\right)$. Since $g^{(6)}(\bar{x})>0$, for all sufficiently large $n$ and all such $x$ we have

$$
\begin{aligned}
n G_{\beta_{n}, K_{n}}\left(x / n^{\kappa}+m_{n}\right)-n G_{\beta_{n}, K_{n}}\left(m_{n}\right) \geq & \frac{1}{2 \cdot 2 !} \cdot g^{(2)}(\bar{x}) x^{2}+\frac{1}{2 \cdot 3 !} \cdot \frac{g^{(3)}(\bar{x})}{n^{u / 2}} x^{3} \quad(8.17) \\
& +\frac{1}{2 \cdot 4 !} \cdot \frac{g^{(4)}(\bar{x})}{n^{u}} x^{4}+\frac{1}{2 \cdot 5 !} \cdot \frac{g^{(5)}(\bar{x})}{n^{3 u / 2}} x^{5} \\
& +\frac{1}{2 \cdot 6 !} \cdot \frac{g^{(6)}(\bar{x})}{n^{2 u}} x^{6}+\frac{1}{n^{2 u}} \mathrm{O}\left(\frac{x}{n^{7 \kappa-1-2 u}}\right) x^{6} \\
\geq & \frac{1}{2 \cdot 2 !} \cdot g^{(2)}(\bar{x}) x^{2}+\frac{1}{2 \cdot 3 !} \cdot \frac{g^{(3)}(\bar{x})}{n^{u / 2}} x^{3} \\
& +\frac{1}{2 \cdot 4 !} \cdot \frac{g^{(4)}(\bar{x})}{n^{u}} x^{4}+\frac{1}{2 \cdot 5 !} \cdot \frac{g^{(5)}(\bar{x})}{n^{3 u / 2}} x^{5} \\
& +\frac{1}{2 \cdot 6 !} \cdot \frac{g^{(6)}(\bar{x})}{n^{2 u}} x^{6}\left(1+\mathrm{O}\left(x / n^{7 \kappa-1-2 u}\right)\right) .
\end{aligned}
$$

By hypothesis (iii') of Theorem 4.1, $\theta \alpha_{0} \in[1 / 6,1 / 2)$. Hence $6 \theta-1 / \alpha_{0} \geq 0$, and so

$$
7 \kappa-1-2 u=\kappa+\left(6 \theta-1 / \alpha_{0}\right) \alpha \geq \kappa
$$

Hence for all $0 \leq x<R n^{\kappa}$ we have $0 \leq x / n^{7 \kappa-1-2 u} \leq x / n^{\kappa}<R$. Thus the term $\mathrm{O}\left(x / n^{7 \kappa-1-2 u}\right)$ appearing in (8.17) can be made larger than -1 for all $0 \leq x / n^{\kappa}<R$ by choosing $R$ to be sufficiently small. Since $g^{(3)}(\bar{x})>0, g^{(4)}(\bar{x})>0, g^{(5)}(\bar{x})>0$, $g^{(6)}(\bar{x})>0$, and $1+\mathrm{O}\left(x / n^{5 \kappa-1-u}\right)>0$, we have that for all sufficiently large $n$ and all $x \in\left[0, R n^{\kappa}\right)$

$$
n G_{\beta_{n}, K_{n}}\left(x / n^{\kappa}+m_{n}\right)-n G_{\beta_{n}, K_{n}}\left(m_{n}\right) \geq \frac{1}{2 \cdot 2 !} g^{(2)}(\bar{x}) x^{2} \geq \frac{1}{8} g^{(2)}(\bar{x}) x^{2} .
$$

This is the conclusion of part (b) of Lemma 8.5 for all $0 \leq x<R n^{\kappa}$ when $g$ has degree 6 .

We now consider $x \in\left(-R n^{\kappa}, 0\right)$. Since $g^{(6)}(\bar{x})>0$, for all sufficiently large $n$ and all such $x$ we have 


$$
\begin{aligned}
n G_{\beta_{n}, K_{n}}\left(x / n^{\kappa}+m_{n}\right)-n G_{\beta_{n}, K_{n}}\left(m_{n}\right) \geq & \frac{1}{2 \cdot 2 !} \cdot g^{(2)}(\bar{x}) x^{2}+\frac{2}{3 !} \cdot \frac{g^{(3)}(\bar{x})}{n^{u / 2}} x^{3} \\
& +\frac{1}{2 \cdot 4 !} \cdot \frac{g^{(4)}(\bar{x})}{n^{u}} x^{4}+\frac{2}{5 !} \cdot \frac{g^{(5)}(\bar{x})}{n^{3 u / 2}} x^{5} \\
& +\frac{1}{2 \cdot 6 !} \cdot \frac{g^{(6)}(\bar{x})}{n^{2 u}} x^{6}+\frac{1}{n^{2 u}} \mathrm{O}\left(\frac{x}{n^{7 \kappa-1-2 u}}\right) x^{6} \\
\geq & \frac{1}{2 \cdot 2 !} \cdot g^{(2)}(\bar{x}) x^{2}+\frac{2}{3 !} \cdot \frac{g^{(3)}(\bar{x})}{n^{u / 2}} x^{3} \\
& +\frac{1}{2 \cdot 4 !} \cdot \frac{g^{(4)}(\bar{x})}{n^{u}} x^{4}+\frac{2}{5 !} \cdot \frac{g^{(5)}(\bar{x})}{n^{3 u / 2}} x^{5} \\
& +\frac{1}{2 \cdot 6 !} \cdot \frac{g^{(6)}(\bar{x})}{n^{2 u}} x^{6}\left(1+\mathrm{O}\left(x / n^{7 \kappa-1-2 u}\right)\right) .
\end{aligned}
$$

Since $7 \kappa-1-2 u \geq \kappa$, for all $-R n^{\kappa}<x<0$ we have $-R<x / n^{\kappa} \leq x / n^{7 \kappa-1-2 u}<$ 0 . Thus the term $\mathrm{O}\left(x / n^{7 \kappa-1-2 u}\right)$ appearing in (8.18) can be made larger than -1 for all $-R<x / n^{\kappa}<0$ by choosing $R$ to be sufficiently small. Since $g^{(4)}(\bar{x})>0, g^{(6)}(\bar{x})>0$, and $1+\mathrm{O}\left(x / n^{7 \kappa-1-2 u}\right)>0$, we have that for all sufficiently large $n$ and all $x \in\left(-R n^{\kappa}, 0\right)$

$$
\begin{aligned}
n G_{\beta_{n}, K_{n}}\left(x / n^{\kappa}+m_{n}\right)-n G_{\beta_{n}, K_{n}}\left(m_{n}\right) & \geq \frac{1}{2 \cdot 2 !} \cdot g^{(2)}(\bar{x}) x^{2}+\frac{2}{3 !} \cdot \frac{g^{(3)}(\bar{x})}{n^{u / 2}} x^{3} \\
& +\frac{2}{5 !} \cdot \frac{g^{(5)}(\bar{x})}{n^{3 u / 2}} x^{5}
\end{aligned}
$$

By Theorem 3.1 we have $m_{n} \sim \bar{x} / n^{\theta \alpha}$. Thus $n^{\theta \alpha} m_{n}=\bar{x}+\varepsilon_{n}$, and

$$
n^{\kappa} m_{n}=n^{u / 2} \cdot n^{\theta \alpha} m_{n}=n^{u / 2}\left(\bar{x}+\varepsilon_{n}\right)
$$

In part (b) of Lemma 8.5 we assume that $x / n^{\kappa}>-(1-\bar{\delta}) m_{n}$ and $0<\bar{\delta}<1$. Thus for all sufficiently large $n$ and all such $x$

$$
x>-(1-\bar{\delta}) n^{\kappa} m_{n}=-(1-\bar{\delta}) n^{u / 2}\left(\bar{x}+\varepsilon_{n}\right) \geq-(1-\bar{\delta}) n^{u / 2} \cdot 2 \bar{x} .
$$

Since $g^{(3)}(\bar{x})>0$ and $g^{(5)}(\bar{x})>0$, we see that for all sufficiently large $n$, all $x \in$ $\left(-R n^{\kappa}, 0\right)$, and all $x / n^{\kappa}>-(1-\bar{\delta}) m_{n}$ there exists $\Delta \in(0,1)$ such that for any $\bar{\delta} \in(\Delta, 1)$ 
the following inequalities hold:

$$
\begin{aligned}
& n G_{\beta_{n}, K_{n}}\left(x / n^{\kappa}+m_{n}\right)-n G_{\beta_{n}, K_{n}}\left(m_{n}\right) \\
& \geq \quad \frac{1}{2 \cdot 2 !} \cdot g^{(2)}(\bar{x}) x^{2}+\frac{2}{3 !} \cdot \frac{g^{(3)}(\bar{x})}{n^{u / 2}} x^{2} \cdot\left[-(1-\bar{\delta}) n^{u / 2} \cdot 2 \bar{x}\right] \\
& \quad+\frac{2}{5 !} \cdot \frac{g^{(5)}(\bar{x})}{n^{3 u / 2}} x^{2} \cdot\left[-(1-\bar{\delta}) n^{u / 2} \cdot 2 \bar{x}\right]^{3} \\
&=\quad\left(\frac{1}{2 \cdot 2 !} \cdot g^{(2)}(\bar{x})-\frac{2 \cdot 2}{3 !} \cdot g^{(3)}(\bar{x})(1-\bar{\delta}) \bar{x}\right. \\
&\left.\quad-\frac{2 \cdot 2^{3}}{5 !} \cdot g^{(5)}(\bar{x})(1-\bar{\delta})^{3} \bar{x}^{3}\right) x^{2} \\
& \geq \frac{1}{2} \cdot \frac{1}{2 \cdot 2 !} \cdot g^{(2)}(\bar{x}) x^{2} \\
&= \frac{1}{8} g^{(2)}(\bar{x}) x^{2} .
\end{aligned}
$$

This is the conclusion of part (b) of Lemma 8.5 for $-R n^{\kappa}<x<0$ and $x / n^{\kappa}>-(1-\bar{\delta}) m_{n}$ when $g$ has degree 6 .

We have shown that for any $\bar{\delta} \in(\Delta, 1)$ there exists $R>0$ such that for all sufficiently large $n$ and all $x \in \mathbb{R}$ satisfying $\left|x / n^{\kappa}\right|<R$ and $x / n^{\kappa}>-(1-\bar{\delta}) m_{n}$

$$
n G_{\beta_{n}, K_{n}}\left(x / n^{\kappa}+m_{n}\right)-n G_{\beta_{n}, K_{n}}\left(m_{n}\right) \geq H(x)=\frac{1}{8} g^{(2)}(\bar{x}) x^{2} .
$$

This completes the proof of part (b) of Lemma 8.5 when $g$ has degree 6. The proof of Lemma 8.5 is complete.

We have completed the statements and proofs of the preparatory lemmas. We now turn to the proof of part (b) of Theorem 6.1. 


\section{CHAPTER 9}

\section{PROOF OF PART (B) OF THEOREM 6.1}

As we saw at the start of subsection 7.1, the proof of part (b) of Theorem 6.1 involves two steps. Step 1 is proved in the next subsection. Step 2 is subdivided into two substeps. Substep 2a is proved in subsection 9.2, and Substep $2 \mathrm{~b}$ is proved in subsection 9.3.

\subsection{Proof of Step 1 in Proof of Theorem 6.1 (b)}

Part (b) of the following lemma states Step 1 in the proof of part (b) of Theorem 6.1. We recall that $W_{n}$ is a sequence of normal random variables with mean 0 and variance $\left(2 \beta_{n} K_{n}\right)^{-1}$ defined on a probability space $(\Omega, \mathcal{F}, Q)$. We denote by $\tilde{E}_{n, \beta_{n}, K_{n}}$ expectation with respect to the product measure $P_{n, \beta_{n}, K_{n}} \times Q ; P_{n, \beta_{n}, K_{n}}$ is defined in (2.1)-(2.2).

Lemma 9.1. We assume that $\left(\beta_{n}, K_{n}\right)$ satisfies the hypotheses of Theorem 4.1 for all $0<$ $\alpha<\alpha_{0}$. For $\bar{\delta} \in(0,1)$ define

$$
A_{n}(\bar{\delta})=\left\{S_{n} / n+W_{n} / n^{1 / 2}>\bar{\delta} m_{n}\right\}
$$

where $m_{n}=m\left(\beta_{n}, K_{n}\right)$. Let $\Delta \in(0,1)$ be the number determined in part (b) of Lemma 8.5. Then for any $0<\alpha<\alpha_{0}$ and any $\bar{\delta} \in(\Delta, 1)$, the following conclusions hold.

(a) We have the limit

$$
\begin{aligned}
\lim _{n \rightarrow \infty} & \int_{-n^{\kappa}(1-\bar{\delta}) m_{n}}^{\infty}|x| \exp \left[-n G_{\beta_{n}, K_{n}}\left(x / n^{\kappa}+m_{n}\right)+n G_{\beta_{n}, K_{n}}\left(m_{n}\right)\right] d x \\
& =\int_{\mathbb{R}}|x| \exp \left[-\frac{1}{2} g^{(2)}(\bar{x}) x^{2}\right] d x .
\end{aligned}
$$


(b) We have the limit

$$
\begin{aligned}
& \lim _{n \rightarrow \infty} \tilde{E}_{n, \beta_{n}, K_{n}}\left\{\left|S_{n} / n^{1-\kappa}+W_{n} / n^{1 / 2-\kappa}-n^{\kappa} m_{n}\right| \mid A_{n}(\bar{\delta})\right\} \\
& \quad=\frac{1}{\int_{\mathbb{R}} \exp \left[-\frac{1}{2} g^{(2)}(\bar{x}) x^{2}\right] d x} \cdot \int_{\mathbb{R}}|x| \exp \left[-\frac{1}{2} g^{(2)}(\bar{x}) x^{2}\right] d x \\
& =\bar{z}
\end{aligned}
$$

Proof of part (a) of Lemma 9.1. Let $\Psi_{n}$ and $\Psi$ denote the measures on $\mathbb{R}$ defined by

$$
\Psi_{n}(d x)=1_{\left(-n^{\kappa}(1-\bar{\delta}) m_{n}, \infty\right)}(x) \cdot \exp \left[-n G_{\beta_{n}, K_{n}}\left(x / n^{\kappa}+m_{n}\right)+n G_{\beta_{n}, K_{n}}\left(m_{n}\right)\right] d x
$$

and

$$
\Psi(d x)=\exp \left[-\frac{1}{2} g^{(2)}(\bar{x}) x^{2}\right] d x
$$

According to Lemma $8.7, \Psi_{n}$ converges weakly to $\Psi$. The limit in part (a) of Lemma 9.1 can be expressed as

$$
\lim _{n \rightarrow \infty} \int_{\mathbb{R}}|x| d \Psi_{n}=\int_{\mathbb{R}}|x| d \Psi
$$

As discussed in Theorem 4 in $§ I I .6$ of [17], this limit would follow from the weak convergence of $\Psi_{n}$ to $\Psi$ if one could prove the uniform integrability estimate

$$
\lim _{j \rightarrow \infty} \sup _{n \in \mathbb{R}} \int_{\{|x|>j\}}|x| d \Psi_{n}=0 .
$$

The next proposition shows that the $\operatorname{limit} \lim _{n \rightarrow \infty} \int_{\mathbb{R}}|x| d \Psi_{n}=\int_{\mathbb{R}}|x| d \Psi$ is a consequence of a condition that is weaker than uniform integrability. 
Proposition 9.2. Let $\Psi_{n}$ be a sequence of measures on $\mathbb{R}$ that converges weakly to a measure $\Psi$ on $\mathbb{R}$. Assume in addition that $\int_{\mathbb{R}}|x| d \Psi<\infty$ and that

$$
\limsup _{j \rightarrow \infty} \int_{n \rightarrow \infty}|x| d \Psi_{n}=0
$$

It then follows that

$$
\lim _{n \rightarrow \infty} \int_{\mathbb{R}}|x| d \Psi_{n}=\int_{\mathbb{R}}|x| d \Psi
$$

Proof. Since $\Psi_{n} \Longrightarrow \Psi$, we have $\Psi_{n}(\mathbb{R}) \rightarrow \Psi(\mathbb{R})$. Hence the proposition is a consequence of Proposition 8.3 in [13] applied to the sequence of probability measures

$$
\frac{1}{\Psi_{n}(\mathbb{R})} \cdot \Psi_{n}(d x) \Longrightarrow \frac{1}{\Psi(\mathbb{R})} \cdot \Psi(d x)
$$

This completes the proof.

We now verify the following hypotheses of Proposition 9.2 for the measures $\Psi_{n}$ and $\Psi$ :

(1) $\Psi_{n} \Longrightarrow \Psi$.

(2) $\int_{\mathbb{R}}|x| d \Psi<\infty$.

(3) $\lim _{j \rightarrow \infty} \lim \sup _{n \rightarrow \infty} \int_{\{|x|>j\}}|x| d \Psi_{n}=0$.

Item (1) is proved in Lemma 8.7, and item (2) is immediate from the definition of $\Psi$. We now prove item (3). Since

$$
\int_{\{|x|>j\}}|x| d \Psi_{n}=\int_{\{|x|>j\} \cap\left\{x>-n^{\kappa}(1-\bar{\delta}) m_{n}\right\}}|x| d \Psi_{n}
$$

we can prove item (3) by showing that

$$
\lim _{j \rightarrow \infty} \limsup _{n \rightarrow \infty} \int_{\{|x|>j\} \cap\left\{x>-n^{\kappa}(1-\bar{\delta}) m_{n}\right\}}|x| d \Psi_{n}=0 .
$$


In order to do this we find, for any $j \in \mathbb{N}$ and all sufficiently large $n$, quantities $A_{j}, B_{n}$ and $C_{n}$ with the properties that

$$
\int_{\{|x|>j\} \cap\left\{x>-n^{\kappa}(1-\bar{\delta}) m_{n}\right\}}|x| d \Psi_{n} \leq A_{j}+B_{n}+C_{n}
$$

$A_{j} \rightarrow 0$ as $j \rightarrow \infty, B_{n} \rightarrow 0$ as $n \rightarrow \infty$, and $C_{n} \rightarrow 0$ as $n \rightarrow \infty$.

We now specify the quantities $A_{j}, B_{n}$ and $C_{n}$. Given positive integers $j$ and $n$, let $R$ and $K$ be positive numbers that satisfy $K>R$ and that will be specified below. Then

$$
\begin{aligned}
\{|x|>j\} \cap\left\{x / n^{\kappa}>-(1-\bar{\delta}) m_{n}\right\} \\
=\left[\{|x|>j\} \cap\left\{\left|x / n^{\kappa}\right|<R\right\} \cap\left\{x / n^{\kappa}>-(1-\bar{\delta}) m_{n}\right\}\right] \\
\cup\left[\{|x|>j\} \cap\left\{R \leq\left|x / n^{\kappa}\right|<K\right\} \cap\left\{x / n^{\kappa}>-(1-\bar{\delta}) m_{n}\right\}\right] \\
\cup\left[\{|x|>j\} \cap\left\{\left|x / n^{\kappa}\right| \geq K\right\} \cap\left\{x / n^{\kappa}>-(1-\bar{\delta}) m_{n}\right\}\right] \\
\subset \quad\left[\{|x|>j\} \cap\left\{\left|x / n^{\kappa}\right|<R\right\} \cap\left\{x / n^{\kappa}>-(1-\bar{\delta}) m_{n}\right\}\right] \\
\cup\left[\left\{R \leq\left|x / n^{\kappa}\right|<K\right\} \cap\left\{x / n^{\kappa}>-(1-\bar{\delta}) m_{n}\right\}\right] \cup\left\{\left|x / n^{\kappa}\right| \geq K\right\} .
\end{aligned}
$$

Since $m_{n} \rightarrow 0$, for all sufficiently large $n$

$$
\left\{R \leq\left|x / n^{\kappa}\right|<K\right\} \cap\left\{x / n^{\kappa}>-(1-\bar{\delta}) m_{n}\right\}=\left\{R \leq x / n^{\kappa}<K\right\}
$$

Hence for all sufficiently large $n$

$$
\begin{aligned}
\int_{\{|x|>j\} \cap\left\{x / n^{\kappa}>-(1-\bar{\delta}) m_{n}\right\}}|x| d \Psi_{n} \\
\leq \int_{\{|x|>j\} \cap\left\{\left|x / n^{\kappa}\right|<R\right\} \cap\left\{x>-n^{\kappa}(1-\bar{\delta}) m_{n}\right\}}|x| d \Psi_{n} \\
\quad+\int_{\left\{R \leq x / n^{\kappa}<K\right\}}|x| d \Psi_{n}+\int_{\left\{\left|x / n^{\kappa}\right| \geq K\right\}}|x| d \Psi_{n} .
\end{aligned}
$$


We next estimate each of these three integrals. By part (b) of Lemma 8.5, there exists $\Delta \in(0,1)$ such that for any $\bar{\delta} \in(\Delta, 1)$ there exists $R>0$ such that for all sufficiently large $\mathrm{n}$ and all $x \in \mathbb{R}$ satisfying $\left|x / n^{\kappa}\right|<R$ and $x / n^{\kappa}>-(1-\bar{\delta}) m_{n}$

$$
n G_{\beta_{n}, K_{n}}\left(x / n^{\kappa}+m_{n}\right)-n G_{\beta_{n}, K_{n}}\left(m_{n}\right) \geq H(x)=\frac{1}{8} g^{(2)}(\bar{x}) x^{2} .
$$

Since $\exp [-H(x)]$ is integrable, for all sufficiently large $n$ we estimate the first integral on the right hand side of equation (9.1) by

$$
\begin{gathered}
\int_{\{|x|>j\} \cap\left\{\left|x / n^{\kappa}\right|<R\right\} \cap\left\{x>-n^{\kappa}(1-\bar{\delta}) m_{n}\right\}}|x| d \Psi_{n} \\
\quad \leq A_{j}=\int_{\{|x|>j\}}|x| \cdot \exp [-H(x)] d x \rightarrow 0 \text { as } j \rightarrow \infty .
\end{gathered}
$$

By part (a) of Lemma 4.4 in [8], there exists $K>0$ and $D_{1}>0$ such that $G_{\beta_{n}, K_{n}}(x) \geq$ $D_{1} x^{2}$ for all $|x|>K$. Since $m_{n} \rightarrow 0$, it follows that for all sufficiently large $n$ and all $x \in \mathbb{R}$ satisfying $\left|x / n^{\kappa}\right| \geq K$, there exists $D>0$ such that

$$
n G_{\beta_{n}, K_{n}}\left(x / n^{\kappa}+m_{n}\right) \geq n D_{1}\left(x / n^{\kappa}+m_{n}\right)^{2} \geq n D\left(x / n^{\kappa}\right)^{2} .
$$

Without loss of generality $K$ can be chosen to be larger than the quantity $R$ specified in the preceding paragraph. By Lemma 8.3, for all sufficiently large $n$ there exists $\varepsilon_{n} \rightarrow 0$ such that

$$
G_{\beta_{n}, K_{n}}\left(m_{n}\right)=\frac{g(\bar{x})+\varepsilon_{n}}{n^{\alpha / \alpha_{0}}} \leq \frac{g(\bar{x})}{2 n^{\alpha / \alpha_{0}}}<0 .
$$

These bounds allow us to estimate the third integral on the right hand side of equation (9.1) by 


$$
\begin{aligned}
& \int_{\left\{\left|x / n^{\kappa}\right| \geq K\right\}}|x| d \Psi_{n} \\
& \quad \leq \int_{\left\{\left|x / n^{\kappa}\right| \geq K\right\}}|x| \exp \left[-n G_{\beta_{n}, K_{n}}\left(x / n^{\kappa}+m_{n}\right)+n G_{\beta_{n}, K_{n}}\left(m_{n}\right)\right] d x \\
& \quad \leq \int_{\left\{\left|x / n^{\kappa}\right| \geq K\right\}}|x| \exp \left[-n D\left(x / n^{\kappa}\right)^{2}\right] d x \\
& \quad \leq C_{n}=\frac{2}{D} \cdot n^{2 \kappa-1} \exp \left[-n D K^{2}\right] \rightarrow 0 \text { as } n \rightarrow \infty .
\end{aligned}
$$

With these choices of $R$ and $K$, we use Lemma 8.6 to estimate the second integral on the right hand side of (9.1). There exists $c_{2}>0$ such that for all sufficiently large $n$

$$
\begin{aligned}
& \int_{\left\{R \leq x / n^{\kappa}<K\right\}}|x| d \Psi_{n} \\
& \quad=\int_{\left\{R \leq x / n^{\kappa}<K\right\}}|x| \exp \left[-n G_{\beta_{n}, K_{n}}\left(x / n^{\kappa}+m_{n}\right)+n G_{\beta_{n}, K_{n}}\left(m_{n}\right)\right] d x \\
& \quad \leq K n^{\kappa} \int_{\left\{x / n^{\kappa} \geq R\right\}} \exp \left[-n G_{\beta_{n}, K_{n}}\left(x / n^{\kappa}+m_{n}\right)+n G_{\beta_{n}, K_{n}}\left(m_{n}\right)\right] d x \\
& \quad \leq B_{n}=K n^{\kappa} \exp \left[-c_{2} n\right] \rightarrow 0 \text { as } n \rightarrow \infty
\end{aligned}
$$

Together equations (9.2), (9.4) and (9.3) prove (9.1). This completes the proof of part (a) of Lemma 9.1.

Proof of part (b) of Lemma 9.1. Part (b) of Lemma 8.2 states that

$$
\begin{aligned}
& \tilde{E}_{n, \beta_{n}, K_{n}}\left\{\left|S_{n} / n^{1-\kappa}+W_{n} / n^{1 / 2-\kappa}-n^{\kappa} m_{n}\right| \mid A_{n}(\bar{\delta})\right\} \\
& \quad=\frac{\int_{-n^{\kappa}(1-\bar{\delta}) m_{n}}^{\infty}|x| \exp \left[-n G_{\beta_{n}, K_{n}}\left(x / n^{\kappa}+m_{n}\right)+n G_{\beta_{n}, K_{n}}\left(m_{n}\right)\right] d x}{\int_{-n^{\kappa}(1-\bar{\delta}) m_{n}}^{\infty} \exp \left[-n G_{\beta_{n}, K_{n}}\left(x / n^{\kappa}+m_{n}\right)+n G_{\beta_{n}, K_{n}}\left(m_{n}\right)\right] d x} .
\end{aligned}
$$

Hence by part (a) of Lemma 9.1 and Lemma 8.7 for $f(x) \equiv 1$, the last integral has the limit

$$
\frac{\int_{\mathbb{R}}|x| \exp \left[-\frac{1}{2} g^{(2)}(\bar{x}) x^{2}\right] d x}{\int_{\mathbb{R}} \exp \left[-\frac{1}{2} g^{(2)}(\bar{x}) x^{2}\right] d x}=\bar{z} .
$$

This completes the proof of part (b) of Lemma 9.1 and hence the proof of the lemma. 
Having completed Step 1 in the proof of part (b) of Theorem 6.1, we now turn to Substep 2a.

\subsection{Proof of Substep 2a in Proof of Theorem 6.1 (b)}

Lemma 9.3 proves Substep 2a of part (b) of Theorem 6.1. We recall that $W_{n}$ is a sequence of normal random variables with mean 0 and variance $\left(2 \beta_{n} K_{n}\right)^{-1}$ defined on a probability space $(\Omega, \mathcal{F}, Q)$. We denote by $\tilde{E}_{n, \beta_{n}, K_{n}}$ expectation with respect to the product measure $P_{n, \beta_{n}, K_{n}} \times Q$.

Lemma 9.3. We assume that $\left(\beta_{n}, K_{n}\right)$ satisfies the hypotheses of Theorem 4.1 for all $0<$ $\alpha<\alpha_{0}$. Denote $m_{n}=m\left(\beta_{n}, K_{n}\right)$. For $\delta \in(0,1)$ define

$$
C_{n}=\tilde{E}_{n, \beta_{n}, K_{n}}\left\{\left|S_{n} / n^{1-\kappa}-n^{\kappa} m_{n}\right| \mid S_{n} / n>\delta m_{n}\right\}
$$

and

$$
D_{n}=\tilde{E}_{n, \beta_{n}, K_{n}}\left\{\left|S_{n} / n^{1-\kappa}+W_{n} / n^{1 / 2-\kappa}-n^{\kappa} m_{n}\right| \mid S_{n} / n>\delta m_{n}\right\}
$$

Then $\lim _{n \rightarrow \infty}\left|C_{n}-D_{n}\right|=0$.

Proof. By part (b) of Lemma 8.8 there exists a constant $c_{1}>0$ such that for all $n$

$$
\tilde{E}_{n, \beta_{n}, K_{n}}\left\{\left|W_{n} / n^{1 / 2-\kappa}\right|\right\} \leq \sqrt{c_{1}} / n^{1 / 2-\kappa}
$$

By Lemma 6.3

$$
\lim _{n \rightarrow \infty} P_{n, \beta_{n}, K_{n}}\left\{S_{n} / n>\delta m_{n}\right\}=1 / 2 .
$$

It follows that there exists a constant $c_{2}>0$ such that for all sufficiently large $n$

$$
\begin{aligned}
\tilde{E}_{n, \beta_{n}, K_{n}}\left\{\left|W_{n} / n^{1 / 2-\kappa}\right| \mid S_{n} / n>\delta m_{n}\right\} & =\frac{\tilde{E}_{n, \beta_{n}, K_{n}}\left\{\left|W_{n} / n^{1 / 2-\kappa}\right| \cdot 1_{\left\{S_{n} / n>\delta m_{n}\right\}}\right\}}{P_{n, \beta_{n}, K_{n}}\left\{S_{n} / n>\delta m_{n}\right\}} \\
& \leq c_{2} / n^{1 / 2-\kappa} .
\end{aligned}
$$


Since

$$
\begin{aligned}
\left|S_{n} / n^{1-\kappa}-n^{\kappa} m_{n}\right|-\left|W_{n} / n^{1 / 2-\kappa}\right| & \leq\left|S_{n} / n^{1-\kappa}+W_{n} / n^{1 / 2-\kappa}-n^{\kappa} m_{n}\right| \\
& \leq\left|S_{n} / n^{1-\kappa}-n^{\kappa} m_{n}\right|+\left|W_{n} / n^{1 / 2-\kappa}\right|
\end{aligned}
$$

we have for all sufficiently large $n$

$$
\begin{aligned}
D_{n}+ & c_{2} / n^{1 / 2-\kappa} \\
= & \tilde{E}_{n, \beta_{n}, K_{n}}\left\{\left|S_{n} / n^{1-\kappa}+W_{n} / n^{1 / 2-\kappa}-n^{\kappa} m_{n}\right| \mid S_{n} / n>\delta m_{n}\right\}+c_{2} / n^{1 / 2-\kappa} \\
= & \frac{\int_{\Lambda^{n} \times \Omega}\left|S_{n} / n^{1-\kappa}+W_{n} / n^{1 / 2-\kappa}-n^{\kappa} m_{n}\right| \cdot 1_{\left\{S_{n} / n>\delta m_{n}\right\}} d\left(P_{n, \beta_{n}, K_{n}} \times Q\right)}{E_{n, \beta_{n}, K_{n}}\left\{1_{\left\{S_{n} / n>\delta m_{n}\right\}}\right\}}+c_{2} / n^{1 / 2-\kappa} \\
\geq & \frac{1}{E_{n, \beta_{n}, K_{n}}\left\{1_{\left\{S_{n} / n>\delta m_{n}\right\}}\right\}} \cdot\left(\int_{\Lambda^{n} \times \Omega}\left|S_{n} / n^{1-\kappa}-n^{\kappa} m_{n}\right| \cdot 1_{\left\{S_{n} / n>\delta m_{n}\right\}} d\left(P_{n, \beta_{n}, K_{n}} \times Q\right)\right. \\
& \left.-\int_{\Lambda^{n} \times \Omega}\left|W_{n} / n^{1 / 2-\kappa}\right| \cdot 1_{\left\{S_{n} / n>\delta m_{n}\right\}} d\left(P_{n, \beta_{n}, K_{n}} \times Q\right)\right) \\
& +\tilde{E}_{n, \beta_{n}, K_{n}}\left\{\left|W_{n} / n^{1 / 2-\kappa}\right| \mid S_{n} / n>\delta m_{n}\right\} \\
= & E_{n, \beta_{n}, K_{n}}\left\{\left|S_{n} / n^{1-\kappa}-n^{\kappa} m_{n}\right| \mid S_{n} / n>\delta m_{n}\right\}=C_{n}
\end{aligned}
$$

and

$$
\begin{aligned}
D_{n}- & c_{2} / n^{1 / 2-\kappa} \\
= & \tilde{E}_{n, \beta_{n}, K_{n}}\left\{\left|S_{n} / n^{1-\kappa}+W_{n} / n^{1 / 2-\kappa}-n^{\kappa} m_{n}\right| \mid S_{n} / n>\delta m_{n}\right\}-c_{2} / n^{1 / 2-\kappa} \\
= & \frac{\int_{\Lambda^{n} \times \Omega}\left|S_{n} / n^{1-\kappa}+W_{n} / n^{1 / 2-\kappa}-n^{\kappa} m_{n}\right| \cdot 1_{\left\{S_{n} / n>\delta m_{n}\right\}} d\left(P_{n, \beta_{n}, K_{n}} \times Q\right)}{E_{n, \beta_{n}, K_{n}}\left\{1_{\left\{S_{n} / n>\delta m_{n}\right\}}\right\}}-c_{2} / n^{1 / 2-\kappa} \\
\leq & \frac{1}{E_{n, \beta_{n}, K_{n}}\left\{1_{\left\{S_{n} / n>\delta m_{n}\right\}}\right\}} \cdot\left(\int_{\Lambda^{n} \times \Omega}\left|S_{n} / n^{1-\kappa}-n^{\kappa} m_{n}\right| \cdot 1_{\left\{S_{n} / n>\delta m_{n}\right\}} d\left(P_{n, \beta_{n}, K_{n}} \times Q\right)\right. \\
& \left.+\int_{\Lambda^{n} \times \Omega}\left|W_{n} / n^{1 / 2-\kappa}\right| \cdot 1_{\left\{S_{n} / n>\delta m_{n}\right\}} d\left(P_{n, \beta_{n}, K_{n}} \times Q\right)\right) \\
& -\tilde{E}_{n, \beta_{n}, K_{n}}\left\{\left|W_{n} / n^{1 / 2-\kappa}\right| \mid S_{n} / n>\delta m_{n}\right\} \\
= & E_{n, \beta_{n}, K_{n}}\left\{\left|S_{n} / n^{1-\kappa}-n^{\kappa} m_{n}\right| \mid S_{n} / n>\delta m_{n}\right\}=C_{n} .
\end{aligned}
$$


Thus we obtain for any $\delta \in(0,1)$ and all sufficiently large $n$

$$
D_{n}+c_{2} / n^{1 / 2-\kappa} \geq C_{n} \geq D_{n}-c_{2} / n^{1 / 2-\kappa}
$$

Since $\kappa<1 / 2$ [Thm. 6.1(c)], it follows that $\lim _{n \rightarrow \infty}\left|C_{n}-D_{n}\right|=0$. This completes the proof of Lemma 9.3.

Having proved Substep 2a in the proof of part (b) of Theorem 6.1, we next turn to Substep 2b.

\subsection{Proof of Substep 2b in Proof of Theorem 6.1 (b)}

We recall that $W_{n}$ is a sequence of normal random variables with mean 0 and variance $\left(2 \beta_{n} K_{n}\right)^{-1}$ defined on a probability space $(\Omega, \mathcal{F}, Q)$. We denote by $\tilde{E}_{n, \beta_{n}, K_{n}}$ expectation with respect to the product measure $P_{n, \beta_{n}, K_{n}} \times Q$. Substep $2 \mathrm{~b}$ in the proof of part (b) of Theorem 6.1 states the following:

$$
\begin{aligned}
\lim _{n \rightarrow \infty} & \tilde{E}_{n, \beta_{n}, K_{n}}\left\{\left|S_{n} / n^{1-\kappa}+W_{n} / n^{1 / 2-\kappa}-n^{\kappa} m_{n}\right| \mid S_{n} / n>\delta m_{n}\right\} \\
= & \lim _{n \rightarrow \infty} \tilde{E}_{n, \beta_{n}, K_{n}}\left\{\left|S_{n} / n^{1-\kappa}+W_{n} / n^{1 / 2-\kappa}-n^{\kappa} m_{n}\right| \mid S_{n} / n+W_{n} / n^{1 / 2}>\delta m_{n}\right\} \\
= & \frac{1}{\int_{\mathbb{R}} \exp \left[-\frac{1}{2} g^{(2)}(\bar{x}) x^{2}\right] d x} \cdot \int_{\mathbb{R}}|x| \exp \left[-\frac{1}{2} g^{(2)}(\bar{x}) x^{2}\right] d x=\bar{z} .
\end{aligned}
$$

This will be proved in Lemma 9.4.

Part (a) of Lemma 9.4 relates the expectation of $\left|S_{n} / n^{1-\kappa}+W_{n} / n^{1 / 2-\kappa}-n^{\kappa} m_{n}\right|$ conditioned on the event $\left\{S_{n} / n>\delta m_{n}\right\}$ to the expectation of the same random variable conditioned on the event $A_{n}(\bar{\delta})$ for two choices of $\bar{\delta}$. Part (b) of the next lemma proves (9.7). The hypotheses of this lemma coincide with the hypotheses of Lemma 8.9 together with the additional condition $\zeta>\frac{1}{2}\left(1-\alpha / \alpha_{0}\right)$, which is used to prove $\Theta_{n, 1} \rightarrow 0$, $\Theta_{n, 3} \rightarrow 0, \Gamma_{n, 1} \rightarrow 0$, and $\Gamma_{n, 3} \rightarrow 0$ in part (a). According to part (c) of Theorem 6.1, 
$\frac{1}{2}\left(1-\alpha / \alpha_{0}\right)+\theta \alpha=\kappa<1 / 2$, which implies $\frac{1}{2}\left(1-\alpha / \alpha_{0}\right)<\frac{1}{2}-\theta \alpha$. This additional condition on $\zeta$ is consistent with the hypothesis on $\zeta$ in Lemma 8.9, which is $0<\zeta<\frac{1}{2}-\theta \alpha$.

Lemma 9.4. We assume that $\left(\beta_{n}, K_{n}\right)$ satisfies the hypotheses of Theorem 4.1 for all $0<$ $\alpha<\alpha_{0}$. For $\bar{\delta} \in(0,1)$ define

$$
A_{n}(\bar{\delta})=\left\{S_{n} / n+W_{n} / n^{1 / 2}>\bar{\delta} m_{n}\right\}
$$

where $m_{n}=m\left(\beta_{n}, K_{n}\right)$. Let $\Delta \in(0,1)$ be the number determined in part (b) of Lemma 8.5. Assume that $0<\alpha<\alpha_{0}$ and choose any numbers $\delta_{1}, \delta, \delta_{2}$ and $\zeta$ satisfying $\Delta<\delta_{1}<$ $\delta<\delta_{2}<1$ and $\zeta \in\left(\frac{1}{2}\left(1-\alpha / \alpha_{0}\right), \frac{1}{2}-\theta \alpha\right)$. The following conclusions hold.

(a) There exists sequences $\Theta_{n, 1} \rightarrow 0, \Theta_{n, 2} \rightarrow 1, \Theta_{n, 3} \rightarrow 0, \Gamma_{n, 1} \rightarrow 0, \Gamma_{n, 2} \rightarrow 1$, and $\Gamma_{n, 3} \rightarrow 0$ such that for all sufficiently large $n$

$$
\begin{aligned}
& \frac{\tilde{E}_{n, \beta_{n}, K_{n}}\left\{\left|S_{n} / n^{1-\kappa}+W_{n} / n^{1 / 2-\kappa}-n^{\kappa} m_{n}\right| \mid A_{n}\left(\delta_{1}\right)\right\}+\Theta_{n, 1}}{\Theta_{n, 2}-\Theta_{n, 3}} \\
& \quad \geq \tilde{E}_{n, \beta_{n}, K_{n}}\left\{\left|S_{n} / n^{1-\kappa}+W_{n} / n^{1 / 2-\kappa}-n^{\kappa} m_{n}\right| \mid S_{n} / n>\delta m_{n}\right\} \\
& \quad \geq \frac{\tilde{E}_{n, \beta_{n}, K_{n}}\left\{\left|S_{n} / n^{1-\kappa}+W_{n} / n^{1 / 2-\kappa}-n^{\kappa} m_{n}\right| \mid A_{n}\left(\delta_{2}\right)\right\}-\Gamma_{n, 1}}{\Gamma_{n, 2}+\Gamma_{n, 3}} .
\end{aligned}
$$

(b) We have the conditional limit

$$
\begin{array}{r}
\lim _{n \rightarrow \infty} \tilde{E}_{n, \beta_{n}, K_{n}}\left\{\left|S_{n} / n^{1-\kappa}+W_{n} / n^{1 / 2-\kappa}-n^{\kappa} m_{n}\right| \mid S_{n} / n>\delta m_{n}\right\} \\
=\lim _{n \rightarrow \infty} \tilde{E}_{n, \beta_{n}, K_{n}}\left\{\left|S_{n} / n^{1-\kappa}+W_{n} / n^{1 / 2-\kappa}-n^{\kappa} m_{n}\right| \mid A_{n}(\delta)\right\} \\
=\frac{1}{\int_{\mathbb{R}} \exp \left[-\frac{1}{2} g^{(2)}(\bar{x}) x^{2}\right] d x} \cdot \int_{\mathbb{R}}|x| \exp \left[-\frac{1}{2} g^{(2)}(\bar{x}) x^{2}\right] d x=\bar{z} .
\end{array}
$$

Proof of part (a) of Lemma 9.4. The hypotheses of this lemma are a subset of the hypotheses of Lemma 8.9. We start by proving the first inequality in part (a). By the first 
inequality in part (b) of Lemma 8.9 and the second inequality in part (a) of Lemma 8.9 we have for all sufficiently large $n$

$$
\begin{aligned}
& \tilde{E}_{n, \beta_{n}, K_{n}}\left\{\left|S_{n} / n^{1-\kappa}+W_{n} / n^{1 / 2-\kappa}-n^{\kappa} m_{n}\right| \mid S_{n} / n>\delta m_{n}\right\} \\
& =\frac{\tilde{E}_{n, \beta_{n}, K_{n}}\left\{\left|S_{n} / n^{1-\kappa}+W_{n} / n^{1 / 2-\kappa}-n^{\kappa} m_{n}\right| \cdot 1_{\left\{S_{n} / n>\delta m_{n}\right\}}\right\}}{P_{n, \beta_{n}, K_{n}}\left\{S_{n} / n>\delta m_{n}\right\}} \\
& \leq \frac{\tilde{E}_{n, \beta_{n}, K_{n}}\left\{\left|S_{n} / n^{1-\kappa}+W_{n} / n^{1 / 2-\kappa}-n^{\kappa} m_{n}\right| \cdot 1_{A_{n}\left(\delta_{1}\right)}\right\}+2 n^{\kappa} e^{-c n^{2 \zeta}}+c_{2} n^{\kappa-1 / 2} e^{-c n^{2 \zeta} / 2}}{\left(P_{n, \beta_{n}, K_{n}} \times Q\right)\left\{A_{n}\left(\delta_{2}\right)\right\}-e^{-c n^{2 \zeta}}} \\
& =\frac{\frac{\tilde{E}_{n, \beta_{n}, K_{n}}\left\{\left|S_{n} / n^{1-\kappa}+W_{n} / n^{1 / 2-\kappa}-n^{\kappa} m_{n}\right| \cdot 1_{\left.A_{n}\left(\delta_{1}\right)\right\}}\right.}{\left(P_{n, \beta_{n}, K_{n}} \times Q\right)\left\{A_{n}\left(\delta_{1}\right)\right\}}+\frac{2 n^{\kappa} e^{-c n^{2 \zeta}}+c_{2} n^{\kappa-1 / 2} e^{-c n^{2 \zeta} / 2}}{\left(P_{n, \beta_{n}}, K_{n} \times Q\right)\left\{A_{n}\left(\delta_{1}\right)\right\}}}{\frac{\left(P_{n, \beta_{n}, K_{n}} \times Q\right)\left\{A_{n}\left(\delta_{2}\right)\right\}}{\left(P_{n, \beta_{n}, K_{n}} \times Q\right)\left\{A_{n}\left(\delta_{1}\right)\right\}}-\frac{e^{-c n^{2 \zeta}}}{\left(P_{n, \beta_{n}, K_{n}} \times Q\right)\left\{A_{n}\left(\delta_{1}\right)\right\}}} \\
& =\frac{\tilde{E}_{n, \beta_{n}, K_{n}}\left\{\left|S_{n} / n^{1-\kappa}+W_{n} / n^{1 / 2-\kappa}-n^{\kappa} m_{n}\right| \mid A_{n}\left(\delta_{1}\right)\right\}+\Theta_{n, 1}}{\Theta_{n, 2}-\Theta_{n, 3}},
\end{aligned}
$$

where $c>0$ and $c_{2}>0$ are constants and

$$
\begin{gathered}
\Theta_{n, 1}=\frac{2 n^{\kappa} e^{-c n^{2 \zeta}}+c_{2} n^{\kappa-1 / 2} e^{-c n^{2 \zeta} / 2}}{\left(P_{n, \beta_{n}, K_{n}} \times Q\right)\left\{A_{n}\left(\delta_{1}\right)\right\}}, \\
\Theta_{n, 2}=\frac{\left(P_{n, \beta_{n}, K_{n}} \times Q\right)\left\{A_{n}\left(\delta_{2}\right)\right\}}{\left(P_{n, \beta_{n}, K_{n}} \times Q\right)\left\{A_{n}\left(\delta_{1}\right)\right\}},
\end{gathered}
$$

and

$$
\Theta_{n, 3}=\frac{e^{-c n^{2 \zeta}}}{\left(P_{n, \beta_{n}, K_{n}} \times Q\right)\left\{A_{n}\left(\delta_{1}\right)\right\}} .
$$

We prove the first inequality in part (a) of the present lemma by showing that, as $n \rightarrow$ $\infty, \Theta_{n, 1} \rightarrow 0, \Theta_{n, 2} \rightarrow 1$ and $\Theta_{n, 3} \rightarrow 0$. These limits hold for any $0<\delta_{1}<1$ and $0<\delta_{2}<1$. By (8.2) in part (a) of Lemma 8.2 with $\bar{\delta}=\delta_{1}$

$$
\begin{aligned}
\Theta_{n, 1} & =\frac{2 n^{\kappa} e^{-c n^{2 \zeta}}+c_{2} n^{\kappa-1 / 2} e^{-c n^{2 \zeta} / 2}}{\left(P_{n, \beta_{n}, K_{n}} \times Q\right)\left\{A_{n}\left(\delta_{1}\right)\right\}} \\
& =\frac{2 n^{\kappa} e^{-c n^{2 \zeta}}+c_{2} n^{\kappa-1 / 2} e^{-c n^{2 \zeta} / 2}}{\int_{-n^{\kappa}\left(1-\delta_{1}\right) m_{n}}^{\infty} \exp \left[-n G_{\beta_{n}, K_{n}}\left(x / n^{\kappa}+m_{n}\right)\right] d x / Z_{n, \kappa}} \\
& =\frac{\exp \left[n G_{\beta_{n}, K_{n}}\left(m_{n}\right)\right] \cdot Z_{n, \kappa} \cdot\left(2 n^{\kappa} e^{-c n^{2 \zeta}}+c_{2} n^{\kappa-1 / 2} e^{-c n^{2 \zeta} / 2}\right)}{\int_{-n^{\kappa}\left(1-\delta_{1}\right) m_{n}}^{\infty} \exp \left[-n G_{\beta_{n}, K_{n}}\left(x / n^{\kappa}+m_{n}\right)+n G_{\beta_{n}, K_{n}}\left(m_{n}\right)\right] d x}
\end{aligned}
$$


We now use Lemma 8.7 with $f \equiv 1$ and $\bar{\delta}=\delta_{1}$. This gives

$$
\begin{aligned}
\lim _{n \rightarrow \infty} \int_{-n^{\kappa}\left(1-\delta_{1}\right) m_{n}}^{\infty} & \exp \left[-n G_{\beta_{n}, K_{n}}\left(x / n^{\kappa}+m_{n}\right)+n G_{\beta_{n}, K_{n}}\left(m_{n}\right)\right] d x \\
= & \int_{\mathbb{R}} \exp \left[-\frac{1}{2} g^{(2)}(\bar{x}) x^{2}\right] d x .
\end{aligned}
$$

By Lemma 8.4, for any $\varepsilon>0$ and all sufficiently large $n$

$$
\begin{aligned}
\exp & {\left[n G_{\beta_{n}, K_{n}}\left(m_{n}\right)\right] \cdot Z_{n, \kappa} \cdot\left(2 n^{\kappa} e^{-c n^{2 \zeta}}+c_{2} n^{\kappa-1 / 2} e^{-c n^{2 \zeta} / 2}\right) } \\
& \leq 2 n^{\kappa} \exp \left[\varepsilon n^{1-\alpha / \alpha_{0}}-c n^{2 \zeta}\right]+c_{2} n^{\kappa-1 / 2} \exp \left[\varepsilon n^{1-\alpha / \alpha_{0}}-c n^{2 \zeta} / 2\right]
\end{aligned}
$$

Since by hypothesis $\zeta>\frac{1}{2}\left(1-\alpha / \alpha_{0}\right)$, it follows that

$$
\lim _{n \rightarrow \infty} \exp \left[n G_{\beta_{n}, K_{n}}\left(m_{n}\right)\right] \cdot Z_{n, \kappa} \cdot\left(2 n^{\kappa} e^{-c n^{2 \zeta}}+c_{2} n^{\kappa-1 / 2} e^{-c n^{2 \zeta} / 2}\right)=0
$$

It follows from the last line of (9.11) that

$$
\begin{aligned}
\lim _{n \rightarrow \infty} \Theta_{n, 1} & =\lim _{n \rightarrow \infty} \frac{\exp \left[n G_{\beta_{n}, K_{n}}\left(m_{n}\right)\right] \cdot Z_{n, \kappa} \cdot\left(2 n^{\kappa} e^{-c n^{2 \zeta}}+c_{2} n^{\kappa-1 / 2} e^{-c n^{2 \zeta} / 2}\right)}{\int_{-n^{\kappa}\left(1-\delta_{1}\right) m_{n}}^{\infty} \exp \left[-n G_{\beta_{n}, K_{n}}\left(x / n^{\kappa}+m_{n}\right)+n G_{\beta_{n}, K_{n}}\left(m_{n}\right)\right] d x} \\
& =\frac{0}{\int_{\mathbb{R}} \exp \left[-\frac{1}{2} g^{(2)}(\bar{x}) x^{2}\right] d x}=0,
\end{aligned}
$$

as claimed.

We now prove that $\lim _{n \rightarrow \infty} \Theta_{n, 2}=0$. By (8.2) in part (a) of Lemma 8.2 with $\bar{\delta}=\delta_{1}$ and $\bar{\delta}=\delta_{2}$

$$
\begin{aligned}
\Theta_{n, 2} & =\frac{\left(P_{n, \beta_{n}, K_{n}} \times Q\right)\left\{A_{n}\left(\delta_{2}\right)\right\}}{\left(P_{n, \beta_{n}, K_{n}} \times Q\right)\left\{A_{n}\left(\delta_{1}\right)\right\}} \\
& =\frac{\int_{-n^{\kappa}\left(1-\delta_{2}\right) m_{n}}^{\infty} \exp \left[-n G_{\beta_{n}, K_{n}}\left(x / n^{\kappa}+m_{n}\right)\right] d x / Z_{n, \kappa}}{\int_{-n^{\kappa}\left(1-\delta_{1}\right) m_{n}}^{\infty} \exp \left[-n G_{\beta_{n}, K_{n}}\left(x / n^{\kappa}+m_{n}\right)\right] d x / Z_{n, \kappa}} \\
& =\frac{\int_{-n^{\kappa}\left(1-\delta_{2}\right) m_{n}}^{\infty} \exp \left[-n G_{\beta_{n}, K_{n}}\left(x / n^{\kappa}+m_{n}\right)+n G_{\beta_{n}, K_{n}}\left(m_{n}\right)\right] d x}{\int_{-n^{\kappa}\left(1-\delta_{1}\right) m_{n}}^{\infty} \exp \left[-n G_{\beta_{n}, K_{n}}\left(x / n^{\kappa}+m_{n}\right)+n G \beta_{n}, K_{n}\left(m_{n}\right)\right] d x} .
\end{aligned}
$$


By Lemma 8.7 for $f \equiv 1, \bar{\delta}=\delta_{1}$, and $\bar{\delta}=\delta_{2}$, both the numerator and denominator have the same limit $\int_{\mathbb{R}} \exp \left[-\frac{1}{2} g^{(2)}(\bar{x}) x^{2}\right] d x$. It follows that $\lim _{n \rightarrow \infty} \Theta_{n, 2}=1$, as claimed.

We now prove that $\lim _{n \rightarrow \infty} \Theta_{n, 3}=0$. Since $\Theta_{n, 1} \geq \Theta_{n, 3}>0$,

$$
\lim _{n \rightarrow \infty} \Theta_{n, 1}=0 \text { implies } \lim _{n \rightarrow \infty} \Theta_{n, 3}=0
$$

as claimed. This completes the proof of the first inequality in part (a) of the present lemma.

We now prove the second inequality in part (a) of the present lemma. By the second inequality in part (b) of Lemma 8.9 and the first inequality in part (a) of Lemma 8.9 we have for all sufficiently large $n$

$$
\begin{aligned}
& \tilde{E}_{n, \beta_{n}, K_{n}}\left\{\left|S_{n} / n^{1-\kappa}+W_{n} / n^{1 / 2-\kappa}-n^{\kappa} m_{n}\right| \mid S_{n} / n>\delta m_{n}\right\} \\
& =\frac{\tilde{E}_{n, \beta_{n}, K_{n}}\left\{\left|S_{n} / n^{1-\kappa}+W_{n} / n^{1 / 2-\kappa}-n^{\kappa} m_{n}\right| \cdot 1_{\left\{S_{n} / n>\delta m_{n}\right\}}\right\}}{P_{n, \beta_{n}, K_{n}}\left\{S_{n} / n>\delta m_{n}\right\}} \\
& \geq \frac{\tilde{E}_{n, \beta_{n}, K_{n}}\left\{\left|S_{n} / n^{1-\kappa}+W_{n} / n^{1 / 2-\kappa}-n^{\kappa} m_{n}\right| \cdot 1_{A_{n}\left(\delta_{2}\right)}\right\}-2 n^{\kappa} e^{-c n^{2 \zeta}}-c_{2} n^{\kappa-1 / 2} e^{-c n^{2 \zeta} / 2}}{\left(P_{n, \beta_{n}, K_{n}} \times Q\right)\left\{A_{n}\left(\delta_{1}\right)\right\}+e^{-c n^{2 \zeta}}} \\
& =\frac{\frac{\tilde{E}_{n, \beta_{n}, K_{n}}\left\{\left|S_{n} / n^{1-\kappa}+W_{n} / n^{1 / 2-\kappa}-n^{\kappa} m_{n}\right| \cdot 1_{A_{n}\left(\delta_{2}\right)}\right\}}{\left(P_{n, \beta_{n}, K_{n}} \times Q\right)\left\{A_{n}\left(\delta_{2}\right)\right\}}-\frac{2 n^{\kappa} e^{-c n^{2 \zeta}}+c_{2} n^{\kappa-1 / 2} e^{-c n^{2 \zeta} / 2}}{\left(P_{n, \beta_{n}, K_{n}} \times Q\right)\left\{A_{n}\left(\delta_{2}\right)\right\}}}{\frac{\left(P_{n, \beta_{n}, K_{n}} \times Q\right)\left\{A_{n}\left(\delta_{1}\right)\right\}}{\left(P_{n, \beta_{n}, K_{n}} \times Q\right)\left\{A_{n}\left(\delta_{2}\right)\right\}}+\frac{e^{-c n^{2 \zeta}}}{\left(P_{n, \beta_{n}, K_{n}} \times Q\right)\left\{A_{n}\left(\delta_{2}\right)\right\}}} \\
& =\frac{\tilde{E}_{n, \beta_{n}, K_{n}}\left\{\left|S_{n} / n^{1-\kappa}+W_{n} / n^{1 / 2-\kappa}-n^{\kappa} m_{n}\right| \mid A_{n}\left(\delta_{2}\right)\right\}-\Gamma_{n, 1}}{\Gamma_{n, 2}+\Gamma_{n, 3}},
\end{aligned}
$$

where $c>0$ and $c_{2}>0$ are constants and

$$
\begin{gathered}
\Gamma_{n, 1}=\frac{2 n^{\kappa} e^{-c n^{2 \zeta}}+c_{2} n^{\kappa-1 / 2} e^{-c n^{2 \zeta} / 2}}{\left(P_{n, \beta_{n}, K_{n}} \times Q\right)\left\{A_{n}\left(\delta_{2}\right)\right\}}, \\
\Gamma_{n, 2}=\frac{\left(P_{n, \beta_{n}, K_{n}} \times Q\right)\left\{A_{n}\left(\delta_{1}\right)\right\}}{\left(P_{n, \beta_{n}, K_{n}} \times Q\right)\left\{A_{n}\left(\delta_{2}\right)\right\}},
\end{gathered}
$$

and

$$
\Gamma_{n, 3}=\frac{e^{-c n^{2 \zeta}}}{\left(P_{n, \beta_{n}, K_{n}} \times Q\right)\left\{A_{n}\left(\delta_{2}\right)\right\}} .
$$

The sequences $\Gamma_{n, 1}, \Gamma_{n, 2}$, and $\Gamma_{n, 3}$ are obtained from $\Theta_{n, 1}, \Theta_{n, 2}$, and $\Theta_{n, 3}$ in (9.8)-(9.10) by interchanging $\delta_{1}$ and $\delta_{2}$. Hence the limits $\Gamma_{n, 1} \rightarrow 0, \Gamma_{n, 2} \rightarrow 1$, and $\Gamma_{n, 3} \rightarrow 0$ follow 
from the limits $\Theta_{n, 1} \rightarrow 0, \Theta_{n, 2} \rightarrow 1$, and $\Theta_{n, 3} \rightarrow 0$, which hold for any $0<\delta_{1}<1$ and $0<\delta_{2}<1$. The proof of part (a) of Lemma 9.4 is complete.

Proof of part (b) of Lemma 9.4. We know from part (b) of Lemma 9.1 that, as $n \rightarrow \infty$, for any $\bar{\delta}=\delta_{1}$ and $\bar{\delta}=\delta_{2}, \quad \tilde{E}_{n, \beta_{n}, K_{n}}\left\{\left|S_{n} / n^{1-\kappa}+W_{n} / n^{1 / 2-\kappa}-n^{\kappa} m_{n}\right| \mid A_{n}(\bar{\delta})\right\}$ has the same limit

$$
\frac{1}{\int_{\mathbb{R}} \exp \left[-\frac{1}{2} g^{(2)}(\bar{x}) x^{2}\right] d x} \cdot \int_{\mathbb{R}}|x| \exp \left[-\frac{1}{2} g^{(2)}(\bar{x}) x^{2}\right] d x=\bar{z} .
$$

By sending $n \rightarrow \infty$ in the inequality in part (a), we have

$$
\begin{aligned}
\bar{z} & =\lim _{n \rightarrow \infty} \tilde{E}_{n, \beta_{n}, K_{n}}\left\{\left|S_{n} / n^{1-\kappa}+W_{n} / n^{1 / 2-\kappa}-n^{\kappa} m_{n}\right| \mid A_{n}\left(\delta_{1}\right)\right\} \\
& \geq \limsup _{n \rightarrow \infty} \tilde{E}_{n, \beta_{n}, K_{n}}\left\{\left|S_{n} / n^{1-\kappa}+W_{n} / n^{1 / 2-\kappa}-n^{\kappa} m_{n}\right| \mid S_{n} / n>\delta m_{n}\right\} \\
& \geq \liminf _{n \rightarrow \infty} \tilde{E}_{n, \beta_{n}, K_{n}}\left\{\left|S_{n} / n^{1-\kappa}+W_{n} / n^{1 / 2-\kappa}-n^{\kappa} m_{n}\right| \mid S_{n} / n>\delta m_{n}\right\} \\
& =\lim _{n \rightarrow \infty} \tilde{E}_{n, \beta_{n}, K_{n}}\left\{\left|S_{n} / n^{1-\kappa}+W_{n} / n^{1 / 2-\kappa}-n^{\kappa} m_{n}\right| \mid A_{n}\left(\delta_{2}\right)\right\} \\
& =\bar{z} .
\end{aligned}
$$

Because the first and last terms in this display are the same, it follows that

$$
\begin{array}{r}
\lim _{n \rightarrow \infty} \tilde{E}_{n, \beta_{n}, K_{n}}\left\{\left|S_{n} / n^{1-\kappa}+W_{n} / n^{1 / 2-\kappa}-n^{\kappa} m_{n}\right| \mid S_{n} / n>\delta m_{n}\right\} \\
=\frac{1}{\int_{\mathbb{R}} \exp \left[-\frac{1}{2} g^{(2)}(\bar{x}) x^{2}\right] d x} \cdot \int_{\mathbb{R}}|x| \exp \left[-\frac{1}{2} g^{(2)}(\bar{x}) x^{2}\right] d x=\bar{z} .
\end{array}
$$

On the other hand, by part (b) of Lemma 9.1 with $\bar{\delta}=\delta$

$$
\begin{aligned}
& \lim _{n \rightarrow \infty} \tilde{E}_{n, \beta_{n}, K_{n}}\left\{\left|S_{n} / n^{1-\kappa}+W_{n} / n^{1 / 2-\kappa}-n^{\kappa} m_{n}\right| \mid A_{n}(\delta)\right\} \\
& \quad=\frac{1}{\int_{\mathbb{R}} \exp \left[-\frac{1}{2} g^{(2)}(\bar{x}) x^{2}\right] d x} \cdot \int_{\mathbb{R}}|x| \exp \left[-\frac{1}{2} g^{(2)}(\bar{x}) x^{2}\right] d x=\bar{z}
\end{aligned}
$$

The proof of part (b) of Lemma 9.4 is complete. 
We now put together the pieces to complete the proof of part (b) of Theorem 6.1. Let $\delta$ be any number satisfying $\Delta<\delta<1$, where $\Delta \in(0,1)$ is determined in part (b) of Lemma 8.5. The proof of part (b) of Theorem 6.1 is divided into Step 1, Substep 2a, and Substep 2b. Step 1 is done in part (b) of Lemma 9.1. There we prove that with $\bar{\delta}=\delta$

$$
\begin{gathered}
\lim _{n \rightarrow \infty} \tilde{E}_{n, \beta_{n}, K_{n}}\left\{\left|S_{n} / n^{1-\kappa}+W_{n} / n^{1 / 2-\kappa}-n^{\kappa} m_{n}\right| \mid S_{n} / n+W_{n} / n^{1 / 2}>\delta m_{n}\right\} \\
=\frac{1}{\int_{\mathbb{R}} \exp \left[-\frac{1}{2} g^{(2)}(\bar{x}) x^{2}\right] d x} \cdot \int_{\mathbb{R}}|x| \exp \left[-\frac{1}{2} g^{(2)}(\bar{x}) x^{2}\right] d x=\bar{z} .
\end{gathered}
$$

Substep $2 \mathrm{a}$ is done in Lemma 9.3. There we prove that $\lim _{n \rightarrow \infty}\left|C_{n}-D_{n}\right|=0$, where

$$
C_{n}=E_{n, \beta_{n}, K_{n}}\left\{\left|S_{n} / n^{1-\kappa}-n^{\kappa} m_{n}\right| \mid S_{n} / n>\delta m_{n}\right\}
$$

and

$$
D_{n}=\tilde{E}_{n, \beta_{n}, K_{n}}\left\{\left|S_{n} / n^{1-\kappa}+W_{n} / n^{1 / 2-\kappa}-n^{\kappa} m_{n}\right| \mid S_{n} / n>\delta m_{n}\right\} .
$$

Substep 2 b is done in part (b) of Lemma 9.4. There we prove that

$$
\begin{aligned}
\lim _{n \rightarrow \infty} & D_{n} \\
= & \lim _{n \rightarrow \infty} \tilde{E}_{n, \beta_{n}, K_{n}}\left\{\left|S_{n} / n^{1-\kappa}+W_{n} / n^{1 / 2-\kappa}-n^{\kappa} m_{n}\right| \mid S_{n} / n+W_{n} / n^{1 / 2}>\delta m_{n}\right\} \\
= & \frac{1}{\int_{\mathbb{R}} \exp \left[-\frac{1}{2} g^{(2)}(\bar{x}) x^{2}\right] d x} \cdot \int_{\mathbb{R}}|x| \exp \left[-\frac{1}{2} g^{(2)}(\bar{x}) x^{2}\right] d x=\bar{z} .
\end{aligned}
$$

Combining these limits yields

$$
\begin{aligned}
\lim _{n \rightarrow \infty} & E_{n, \beta_{n}, K_{n}}\left\{\left|S_{n} / n^{1-\kappa}-n^{\kappa} m_{n}\right| \mid S_{n} / n>\delta m_{n}\right\} \\
& =\lim _{n \rightarrow \infty} \tilde{E}_{n, \beta_{n}, K_{n}}\left\{\left|S_{n} / n^{1-\kappa}+W_{n} / n^{1 / 2-\kappa}-n^{\kappa} m_{n}\right| \mid S_{n} / n+W_{n} / n^{1 / 2}>\delta m_{n}\right\} \\
& =\frac{1}{\int_{\mathbb{R}} \exp \left[-\frac{1}{2} g^{(2)}(\bar{x}) x^{2}\right] d x} \cdot \int_{\mathbb{R}}|x| \exp \left[-\frac{1}{2} g^{(2)}(\bar{x}) x^{2}\right] d x=\bar{z} .
\end{aligned}
$$


This gives the conditional limit stated in part (b) of Theorem 6.1:

$$
\begin{aligned}
\lim _{n \rightarrow \infty} & n^{\kappa} E_{n, \beta_{n}, K_{n}}\left\{\left|S_{n} / n-m\left(\beta_{n}, K_{n}\right)\right| \mid S_{n} / n>\delta m\left(\beta_{n}, K_{n}\right)\right\} \\
\quad= & \lim _{n \rightarrow \infty} E_{n, \beta_{n}, K_{n}}\left\{\left|S_{n} / n^{1-\kappa}-n^{\kappa} m\left(\beta_{n}, K_{n}\right)\right| \mid S_{n} / n>\delta m\left(\beta_{n}, K_{n}\right)\right\}=\bar{z} .
\end{aligned}
$$

The proof of part (b) of Theorem 6.1 is complete. 


\section{CHAPTER 10}

\section{PROOF OF PART (A) OF THEOREM 6.1}

As we pointed out in subsection 7.2, the proof of part (a) of Theorem 6.1 follows the same pattern of proof as part (b) of Theorem 6.1, the details of which are given in sections 8 and 9. From the outline of the proof of part (a) of Theorem 6.1 given in subsection 7.2, one can easily construct this proof from the material in sections 8 and 9 . In the present section, for reasons of completeness, the details of the proof of part (a) of Theorem 6.1 are given.

As we saw at the start of subsection 7.2, the proof of part (a) of Theorem 6.1 involves two steps. Step 1 is proved in the next subsection. Step 2 is subdivided into two substeps. Substep 2a is proved in subsection 10.2, and Substep $2 \mathrm{~b}$ is proved in subsection 10.3.

\subsection{Proof of Step 1 in Proof of Theorem 6.1 (a)}

The following lemma states Step 1 in the proof of part (a) of Theorem 6.1. We recall that $W_{n}$ is a sequence of normal random variables with mean 0 and variance $\left(2 \beta_{n} K_{n}\right)^{-1}$ defined on a probability space $(\Omega, \mathcal{F}, Q)$. We denote by $\tilde{E}_{n, \beta_{n}, K_{n}}$ expectation with respect to the product measure $P_{n, \beta_{n}, K_{n}} \times Q ; P_{n, \beta_{n}, K_{n}}$ is defined in (2.1)-(2.2).

Lemma 10.1. We assume that $\left(\beta_{n}, K_{n}\right)$ satisfies the hypotheses of Theorem 4.1 for all $0<\alpha<\alpha_{0}$. Given any $\alpha \in\left(0, \alpha_{0}\right)$, define $\kappa=\frac{1}{2}\left(1-\alpha / \alpha_{0}\right)+\theta \alpha$. For $\bar{\delta} \in(0,1)$ define

$$
A_{n}(\bar{\delta})=\left\{S_{n} / n+W_{n} / n^{1 / 2}>\bar{\delta} m_{n}\right\}
$$


where $m_{n}=m\left(\beta_{n}, K_{n}\right)$. Let $f$ be any bounded, uniformly continuous function and let $\Delta \in(0,1)$ be the number determined in part (b) of Lemma 8.5. Then for any $0<\alpha<\alpha_{0}$ and any $\bar{\delta} \in(\Delta, 1)$, we have the limit

$$
\begin{aligned}
\lim _{n \rightarrow \infty} \tilde{E}_{n, \beta_{n}, K_{n}}\left\{f\left(S_{n} / n^{1-\kappa}+W_{n} / n^{1 / 2-\kappa}-n^{\kappa} m_{n}\right) \mid A_{n}(\bar{\delta})\right\} \\
=\frac{1}{\int_{\mathbb{R}} \exp \left[-\frac{1}{2} g^{(2)}(\bar{x}) x^{2}\right] d x} \cdot \int_{\mathbb{R}} f(x) \exp \left[-\frac{1}{2} g^{(2)}(\bar{x}) x^{2}\right] d x .
\end{aligned}
$$

Proof. Applying part (a) of Lemma 8.2 to $h=f$ and multiplying numerator and denominator by $\exp \left[n G_{\beta_{n}, K_{n}}\left(m_{n}\right)\right]$ we have

$$
\begin{aligned}
\lim _{n \rightarrow \infty} & \tilde{E}_{n, \beta_{n}, K_{n}}\left\{f\left(S_{n} / n^{1-\kappa}+W_{n} / n^{1 / 2-\kappa}-n^{\kappa} m_{n}\right) \mid A_{n}(\bar{\delta})\right\} \\
= & \frac{1}{P_{n, \beta_{n}, K_{n}} \times Q\left\{A_{n}(\bar{\delta})\right\}} \cdot \tilde{E}_{n, \beta_{n}, K_{n}}\left\{f\left(S_{n} / n^{1-\kappa}+W_{n} / n^{1 / 2-\kappa}-n^{\kappa} m_{n}\right) \cdot 1_{A_{n}(\bar{\delta})}\right\} \\
= & \lim _{n \rightarrow \infty} \frac{\int_{-n^{\kappa}(1-\bar{\delta}) m_{n}}^{\infty} f(x) \exp \left[-n G_{\beta_{n}, K_{n}}\left(x / n^{\kappa}+m_{n}\right)+n G_{\beta_{n}, K_{n}}\left(m_{n}\right)\right] d x}{\int_{-n^{\kappa}(1-\bar{\delta}) m_{n}}^{\infty} \exp \left[-n G_{\beta_{n}, K_{n}}\left(x / n^{\kappa}+m_{n}\right)+n G_{\beta_{n}, K_{n}}\left(m_{n}\right)\right] d x} \\
= & \frac{1}{\int_{\mathbb{R}} \exp \left[-\frac{1}{2} g^{(2)}(\bar{x}) x^{2}\right] d x} \cdot \int_{\mathbb{R}} f(x) \exp \left[-\frac{1}{2} g^{(2)}(\bar{x}) x^{2}\right] d x .
\end{aligned}
$$

The limits in the preceding two lines are a consequence of the second equality in (8.5) in Lemma 8.7 for the given bounded, uniformly continuous function $f$ and for $f \equiv 1$. The proof of Lemma 10.1 is complete.

Having completed Step 1 in the proof of part (a) of Theorem 6.1, we now turn to Substep $2 a$.

\subsection{Proof of Substep 2a in Proof of Theorem 6.1 (a)}

Lemma 10.2 proves Substep 2a of part (a) of Theorem 6.1. We recall that $W_{n}$ is a sequence of normal random variables with mean 0 and variance $\left(2 \beta_{n} K_{n}\right)^{-1}$ defined on a 
probability space $(\Omega, \mathcal{F}, Q)$. We denote by $\tilde{E}_{n, \beta_{n}, K_{n}}$ expectation with respect to the product measure $P_{n, \beta_{n}, K_{n}} \times Q$.

Lemma 10.2. We assume that $\left(\beta_{n}, K_{n}\right)$ satisfies the hypotheses of Theorem 4.1 for all $0<\alpha<\alpha_{0}$. Denote $m_{n}=m\left(\beta_{n}, K_{n}\right)$. Let $f$ be any bounded, uniformly continuous function. For $\delta \in(0,1)$ define

$$
\tilde{C}_{n}=E_{n, \beta_{n}, K_{n}}\left\{f\left(S_{n} / n^{1-\kappa}-n^{\kappa} m_{n}\right) \mid S_{n} / n>\delta m_{n}\right\}
$$

and

$$
\tilde{D}_{n}=\tilde{E}_{n, \beta_{n}, K_{n}}\left\{f\left(S_{n} / n^{1-\kappa}+W_{n} / n^{1 / 2-\kappa}-n^{\kappa} m_{n}\right) \mid S_{n} / n>\delta m_{n}\right\}
$$

Then we have

$$
\lim _{n \rightarrow \infty}\left|\tilde{C}_{n}-\tilde{D}_{n}\right|=0
$$

Proof. According to part (c) of Theorem 6.1, $0<\kappa<1 / 2$. We apply part (a) of Lemma 8.8 with $\zeta=1 / 2-\kappa>0$. For any $\xi>0$ there exists a constant $c>0$ such that for all $n \in \mathbb{N}$

$$
Q\left\{\left|W_{n} / n^{1 / 2-\kappa}\right|>\xi\right\} \leq \exp \left[-c n^{1-2 \kappa}\right]
$$

Define

$$
y_{n}=S_{n} / n^{1-\kappa}+W_{n} / n^{1 / 2-\kappa}-n^{\kappa} m_{n}, \quad z_{n}=S_{n} / n^{1-\kappa}-n^{\kappa} m_{n}
$$

and

$$
I_{n}=\left|\int_{\mathbb{R}} f\left(y_{n}\right) \cdot 1_{\left\{S_{n} / n>\delta m_{n}\right\}} d\left(P_{n, \beta_{n}, K_{n}} \times Q\right)-\int_{\mathbb{R}} f\left(z_{n}\right) \cdot 1_{\left\{S_{n} / n>\delta m_{n}\right\}} d\left(P_{n, \beta_{n}, K_{n}} \times Q\right)\right| .
$$

Thus

$$
\left|\tilde{C}_{n}-\tilde{D}_{n}\right|=\frac{I_{n}}{P_{n, \beta_{n}, K_{n}}\left\{S_{n} / n>\delta m_{n}\right\}}
$$


For any $\xi>0$ we have

$$
\begin{aligned}
I_{n} \leq & \int_{\mathbb{R}}\left|f\left(y_{n}\right)-f\left(z_{n}\right)\right| \cdot 1_{\left\{S_{n} / n>\delta m_{n}\right\}} d\left(P_{n, \beta_{n}, K_{n}} \times Q\right) \\
= & \int_{\left\{\left|y_{n}-z_{n}\right| \leq \xi\right\}}\left|f\left(y_{n}\right)-f\left(z_{n}\right)\right| \cdot 1_{\left\{S_{n} / n>\delta m_{n}\right\}} d\left(P_{n, \beta_{n}, K_{n}} \times Q\right) \\
& +\int_{\left\{\left|y_{n}-z_{n}\right|>\xi\right\}}\left|f\left(y_{n}\right)-f\left(z_{n}\right)\right| \cdot 1_{\left\{S_{n} / n>\delta m_{n}\right\}} d\left(P_{n, \beta_{n}, K_{n}} \times Q\right) \\
\equiv & I_{n}^{1}+I_{n}^{2},
\end{aligned}
$$

where $I_{n}^{1}$ and $I_{n}^{2}$ represent the integrals in the preceding two lines.

We prove that for any $\varepsilon>0$ there exists $\xi>0$ and $N \in \mathbb{N}$ such that $\forall n>N$

$$
I_{n}^{1} \leq \varepsilon / 2 \quad \text { and } \quad I_{n}^{2} \leq \varepsilon / 2
$$

Since $f$ is uniformly continuous, there exist $\xi \in(0,1)$ such that for all $x, y \in \mathbb{R}$ satisfying $|x-y| \leq \xi$, we have $|f(x)-f(y)| \leq \varepsilon / 2$. Thus for all $n \in \mathbb{N}$

$$
I_{n}^{1} \leq \varepsilon / 2 \cdot \int_{\left\{\left|y_{n}-z_{n}\right| \leq \xi\right\}} 1_{\left\{S_{n} / n>\delta m_{n}\right\}} d\left(P_{n, \beta_{n}, K_{n}} \times Q\right) \leq \varepsilon / 2 .
$$

In addition, by (10.2)

$$
\begin{aligned}
I_{n}^{2} & \leq 2\|f\|_{\infty} \cdot\left(P_{n, \beta_{n}, K_{n}} \times Q\right)\left\{\left\{\left|y_{n}-z_{n}\right|>\xi\right\} \cap\left\{S_{n} / n>\delta m_{n}\right\}\right\} \\
& \leq 2\|f\|_{\infty} \cdot\left(P_{n, \beta_{n}, K_{n}} \times Q\right)\left\{\left|y_{n}-z_{n}\right|>\xi\right\} \\
& =2\|f\|_{\infty} \cdot Q\left\{\left|W_{n} / n^{1 / 2-\kappa}\right|>\xi\right\} \\
& \leq 2\|f\|_{\infty} \cdot \exp \left[-c n^{1-2 \kappa}\right] .
\end{aligned}
$$

According to part (c) of Theorem 6.1, $\kappa<1 / 2$. Thus $\exists N \in \mathbb{N}$ such that $\forall n>N, I_{n}^{2} \leq \varepsilon / 2$. It follows that for $\forall n>N$

$$
I_{n} \leq I_{n}^{1}+I_{n}^{2} \leq \varepsilon
$$


Then for $\forall n>N$

$$
\left|\tilde{C}_{n}-\tilde{D}_{n}\right|=\frac{I_{n}}{P_{n, \beta_{n}, K_{n}}\left\{S_{n} / n>\delta m_{n}\right\}} \leq \frac{\varepsilon}{P_{n, \beta_{n}, K_{n}}\left\{S_{n} / n>\delta m_{n}\right\}}
$$

By Lemma 6.3

$$
\lim _{n \rightarrow \infty} P_{n, \beta_{n}, K_{n}}\left\{S_{n} / n>\delta m_{n}\right\}=1 / 2
$$

Since $\varepsilon>0$ is arbitrary, we have

$$
\lim _{n \rightarrow \infty}\left|\tilde{C}_{n}-\tilde{D}_{n}\right|=0
$$

The proof of lemma is complete.

Having proved Substep 2a in the proof of part (a) of Theorem 6.1, we next turn to Substep 2b.

\subsection{Proof of Substep 2b in Proof of Theorem 6.1 (a)}

We recall that $W_{n}$ is a sequence of normal random variables with mean 0 and variance $\left(2 \beta_{n} K_{n}\right)^{-1}$ defined on a probability space $(\Omega, \mathcal{F}, Q)$. We denote by $\tilde{E}_{n, \beta_{n}, K_{n}}$ expectation with respect to the product measure $P_{n, \beta_{n}, K_{n}} \times Q$. Substep $2 \mathrm{~b}$ in the proof of part (a) of Theorem 6.1 says the following:

$$
\begin{aligned}
\lim _{n \rightarrow \infty} & \tilde{E}_{n, \beta_{n}, K_{n}}\left\{f\left(S_{n} / n^{1-\kappa}+W_{n} / n^{1 / 2-\kappa}-n^{\kappa} m_{n}\right) \mid S_{n} / n>\delta m_{n}\right\} \\
= & \lim _{n \rightarrow \infty} \tilde{E}_{n, \beta_{n}, K_{n}}\left\{f\left(S_{n} / n^{1-\kappa}+W_{n} / n^{1 / 2-\kappa}-n^{\kappa} m_{n}\right) \mid S_{n} / n+W_{n} / n^{1 / 2}>\delta m_{n}\right\} \\
= & \frac{1}{\int_{\mathbb{R}} \exp \left[-\frac{1}{2} g^{(2)}(\bar{x}) x^{2}\right] d x} \cdot \int_{\mathbb{R}} f(x) \exp \left[-\frac{1}{2} g^{(2)}(\bar{x}) x^{2}\right] d x .
\end{aligned}
$$

Substep $2 \mathrm{~b}$ in the proof of part (a) of Theorem 6.1 will be proved in Lemma 10.4. The next lemma does some preparatory work for this. 
Lemma 10.3. We assume that $\left(\beta_{n}, K_{n}\right)$ satisfies the hypotheses of Theorem 4.1 for all $0<\alpha<\alpha_{0}$. For $\bar{\delta} \in(0,1)$ define

$$
A_{n}(\bar{\delta})=\left\{S_{n} / n+W_{n} / n^{1 / 2}>\bar{\delta} m_{n}\right\}
$$

where $m_{n}=m\left(\beta_{n}, K_{n}\right)$. Let $\varphi$ be any nonnegative bounded, uniformly continuous function and let $\Delta \in(0,1)$ be the number determined in part (b) of Lemma 8.5. Assume that $0<\alpha<\alpha_{0}$ and choose any numbers $\delta_{1}, \delta, \delta_{2}$ and $\zeta$ satisfying $\Delta<\delta_{1}<\delta<\delta_{2}<1$ and $\zeta \in\left(0, \frac{1}{2}-\theta \alpha\right)$. Then there exists constant $c>0$ such that for all sufficiently large $n$

$$
\begin{aligned}
& \tilde{E}_{n, \beta_{n}, K_{n}}\left\{\varphi\left(S_{n} / n^{1-\kappa}+W_{n} / n^{1 / 2-\kappa}-n^{\kappa} m_{n}\right) \cdot 1_{A_{n}\left(\delta_{1}\right)}\right\}+\|\varphi\|_{\infty} e^{-c n^{2 \zeta}} \\
& \quad \geq \tilde{E}_{n, \beta_{n}, K_{n}}\left\{\varphi\left(S_{n} / n^{1-\kappa}+W_{n} / n^{1 / 2-\kappa}-n^{\kappa} m_{n}\right) \cdot 1_{\left\{S_{n} / n>\delta m_{n}\right\}}\right\} \\
& \quad \geq \tilde{E}_{n, \beta_{n}, K_{n}}\left\{\varphi\left(S_{n} / n^{1-\kappa}+W_{n} / n^{1 / 2-\kappa}-n^{\kappa} m_{n}\right) \cdot 1_{A_{n}\left(\delta_{2}\right)}\right\}-\|\varphi\|_{\infty} e^{-c n^{2 \zeta}} .
\end{aligned}
$$

Proof of part (a) of Lemma 10.3. We choose $\zeta \in\left(0, \frac{1}{2}-\theta \alpha\right)$. We start by restating the two claims in Lemma 8.9.

Claim 1. For all sufficiently large $n,\left\{S_{n} / n>\delta m_{n}\right\} \subset A_{n}\left(\delta_{1}\right) \cup\left\{\left|W_{n}\right|>\frac{1}{2} n^{\zeta}\right\}$.

Claim 2. For all sufficiently large $n,\left\{S_{n} / n>\delta m_{n}\right\} \supset A_{n}\left(\delta_{2}\right) \backslash\left\{\left|W_{n}\right|>\frac{1}{2} n^{\zeta}\right\}$.

We first use Claim 1 to prove the first inequality. For all sufficiently large $n$ 


$$
\begin{aligned}
& \tilde{E}_{n, \beta_{n}, K_{n}}\left\{\varphi\left(S_{n} / n^{1-\kappa}+W_{n} / n^{1 / 2-\kappa}-n^{\kappa} m_{n}\right) \cdot 1_{\left\{S_{n} / n>\delta m_{n}\right\}}\right\} \\
& =\int_{\Lambda^{n} \times \Omega} \varphi\left(S_{n} / n^{1-\kappa}+W_{n} / n^{1 / 2-\kappa}-n^{\kappa} m_{n}\right) \cdot 1_{\left\{S_{n} / n>\delta m_{n}\right\}} d\left(P_{n, \beta_{n}, K_{n}} \times Q\right) \\
& \leq \int_{\Lambda^{n} \times \Omega} \varphi\left(S_{n} / n^{1-\kappa}+W_{n} / n^{1 / 2-\kappa}-n^{\kappa} m_{n}\right) \cdot 1_{A_{n}\left(\delta_{1}\right) \cup\left\{\left|W_{n}\right|>\frac{1}{2} n \zeta\right\}} d\left(P_{n, \beta_{n}, K_{n}} \times Q\right) \\
& \leq \int_{\Lambda^{n} \times \Omega} \varphi\left(S_{n} / n^{1-\kappa}+W_{n} / n^{1 / 2-\kappa}-n^{\kappa} m_{n}\right) \cdot 1_{A_{n}\left(\delta_{1}\right)} d\left(P_{n, \beta_{n}, K_{n}} \times Q\right) \\
& \quad+\int_{\Lambda^{n} \times \Omega} \varphi\left(S_{n} / n^{1-\kappa}+W_{n} / n^{1 / 2-\kappa}-n^{\kappa} m_{n}\right) \cdot 1_{\left\{\left|W_{n}\right|>\frac{1}{2} n \zeta\right\}} d\left(P_{n, \beta_{n}, K_{n}} \times Q\right) .
\end{aligned}
$$

Using part (a) of Lemma 8.8, for all sufficiently large $n$ we bound the last integral in (10.3) by

$$
\|\varphi\|_{\infty} \cdot Q\left\{\left|W_{n}\right|>\frac{1}{2} n^{\zeta}\right\} \leq\|\varphi\|_{\infty} \cdot \exp \left(-c n^{2 \zeta}\right)
$$

where $c>0$ is a constant. It follows that for all sufficiently large $n$

$$
\begin{aligned}
& \tilde{E}_{n, \beta_{n}, K_{n}}\left\{\varphi\left(S_{n} / n^{1-\kappa}+W_{n} / n^{1 / 2-\kappa}-n^{\kappa} m_{n}\right) \cdot 1_{\left\{S_{n} / n>\delta m_{n}\right\}}\right\} \\
& \quad \leq \tilde{E}_{n, \beta_{n}, K_{n}}\left\{\varphi\left(S_{n} / n^{1-\kappa}+W_{n} / n^{1 / 2-\kappa}-n^{\kappa} m_{n}\right) \cdot 1_{A_{n}\left(\delta_{1}\right)}\right\}+\|\varphi\|_{\infty} e^{-c n^{2 \zeta}} .
\end{aligned}
$$

This completes the proof of the first inequality in part (a).

We now use Claim 2 to prove the second inequality. For all sufficiently large $n$

$$
\begin{aligned}
& \tilde{E}_{n, \beta_{n}, K_{n}}\left\{\varphi\left(S_{n} / n^{1-\kappa}+W_{n} / n^{1 / 2-\kappa}-n^{\kappa} m_{n}\right) \cdot 1_{\left\{S_{n} / n>\delta m_{n}\right\}}\right\} \\
& =\int_{\Lambda^{n} \times \Omega} \varphi\left(S_{n} / n^{1-\kappa}+W_{n} / n^{1 / 2-\kappa}-n^{\kappa} m_{n}\right) \cdot 1_{\left\{S_{n} / n>\delta m_{n}\right\}} d\left(P_{n, \beta_{n}, K_{n}} \times Q\right) \\
& \geq \int_{\Lambda^{n} \times \Omega} \varphi\left(S_{n} / n^{1-\kappa}+W_{n} / n^{1 / 2-\kappa}-n^{\kappa} m_{n}\right) \cdot 1_{A_{n}\left(\delta_{2}\right) \backslash\left\{\left|W_{n}\right|>\frac{1}{2} n \zeta\right\}} d\left(P_{n, \beta_{n}, K_{n}} \times Q\right) \\
& \geq \int_{\Lambda^{n} \times \Omega} \varphi\left(S_{n} / n^{1-\kappa}+W_{n} / n^{1 / 2-\kappa}-n^{\kappa} m_{n}\right) \cdot 1_{A_{n}\left(\delta_{2}\right)} d\left(P_{n, \beta_{n}, K_{n}} \times Q\right) \\
& \quad-\int_{\Lambda^{n} \times \Omega} \varphi\left(S_{n} / n^{1-\kappa}+W_{n} / n^{1 / 2-\kappa}-n^{\kappa} m_{n}\right) \cdot 1_{\left\{\left|W_{n}\right|>\frac{1}{2} n^{\zeta}\right\}} d\left(P_{n, \beta_{n}, K_{n}} \times Q\right) .
\end{aligned}
$$

The last inequality follows from the fact that for any sets $C$ and $D, 1_{C \backslash D} \geq 1_{C}-1_{D}$. The last integral in (10.5) coincides with the last integral in (10.3) and so can be bounded using 
(10.4). Hence from (10.5) we obtain for all sufficiently large $n$

$$
\begin{aligned}
& \tilde{E}_{n, \beta_{n}, K_{n}}\left\{\varphi\left(S_{n} / n^{1-\kappa}+W_{n} / n^{1 / 2-\kappa}-n^{\kappa} m_{n}\right) \cdot 1_{\left\{S_{n} / n>\delta m_{n}\right\}}\right\} \\
& \quad \geq \tilde{E}_{n, \beta_{n}, K_{n}}\left\{\varphi\left(S_{n} / n^{1-\kappa}+W_{n} / n^{1 / 2-\kappa}-n^{\kappa} m_{n}\right) \cdot 1_{A_{n}\left(\delta_{2}\right)}\right\}-\|\varphi\|_{\infty} e^{-c n^{2 \zeta}} .
\end{aligned}
$$

The proof of Lemma 10.3 is complete.

Part (a) of the next lemma relates the expectation of $f\left(S_{n} / n^{1-\kappa}+W_{n} / n^{1 / 2-\kappa}-n^{\kappa} m_{n}\right)$ conditioned on the event $\left\{S_{n} / n>\delta m_{n}\right\}$ to the expectation of the same random variable conditioned on the event $A_{n}(\bar{\delta})$ for two choices of $\bar{\delta}$. Part (b) of the next lemma states Substep $2 \mathrm{~b}$ in the proof of part (a) of Theorem 6.1. The hypotheses of this lemma coincide with the hypotheses of Lemma 8.9 together with the additional condition $\zeta>\frac{1}{2}\left(1-\alpha / \alpha_{0}\right)$, which is used to prove $\tilde{\Theta}_{n, 1} \rightarrow 0, \tilde{\Theta}_{n, 3} \rightarrow 0, \tilde{\Gamma}_{n, 1} \rightarrow 0$, and $\tilde{\Gamma}_{n, 3} \rightarrow 0$ in part (a). According to part (c) of Theorem 6.1, $\frac{1}{2}\left(1-\alpha / \alpha_{0}\right)+\theta \alpha=\kappa<1 / 2$, which implies $\frac{1}{2}\left(1-\alpha / \alpha_{0}\right)<$ $\frac{1}{2}-\theta \alpha$. This additional condition on $\zeta$ is consistent with the hypothesis on $\zeta$ in Lemma 8.9 , which is $0<\zeta<\frac{1}{2}-\theta \alpha$.

Lemma 10.4. We assume that $\left(\beta_{n}, K_{n}\right)$ satisfies the hypotheses of Theorem 4.1 for all $0<\alpha<\alpha_{0}$. For $\bar{\delta} \in(0,1)$ define

$$
A_{n}(\bar{\delta})=\left\{S_{n} / n+W_{n} / n^{1 / 2}>\bar{\delta} m_{n}\right\}
$$

where $m_{n}=m\left(\beta_{n}, K_{n}\right)$. Let $f$ be any bounded, uniformly continuous function, let $\varphi$ be any nonnegative bounded uniformly continuous function, and let $\Delta \in(0,1)$ be the number determined in part (b) of Lemma 8.5. Assume that $0<\alpha<\alpha_{0}$ and choose any numbers $\delta_{1}, \delta, \delta_{2}$ and $\zeta$ satisfying $\Delta<\delta_{1}<\delta<\delta_{2}<1$ and $\zeta \in\left(\frac{1}{2}\left(1-\alpha / \alpha_{0}\right), \frac{1}{2}-\theta \alpha\right)$. Then the following conclusions hold. 
(a) There exists sequences $\tilde{\Theta}_{n, 1} \rightarrow 0, \tilde{\Theta}_{n, 2} \rightarrow 1, \tilde{\Theta}_{n, 3} \rightarrow 0, \tilde{\Gamma}_{n, 1} \rightarrow 0, \tilde{\Gamma}_{n, 2} \rightarrow 1$, and $\tilde{\Gamma}_{n, 3} \rightarrow 0$ such that for all sufficiently large $n$

$$
\begin{gathered}
\frac{\tilde{E}_{n, \beta_{n}, K_{n}}\left\{\varphi\left(S_{n} / n^{1-\kappa}+W_{n} / n^{1 / 2-\kappa}-n^{\kappa} m_{n}\right) \mid A_{n}\left(\delta_{1}\right)\right\}+\tilde{\Theta}_{n, 1}}{\tilde{\Theta}_{n, 2}-\tilde{\Theta}_{n, 3}} \\
\geq \tilde{E}_{n, \beta_{n}, K_{n}}\left\{\varphi\left(S_{n} / n^{1-\kappa}+W_{n} / n^{1 / 2-\kappa}-n^{\kappa} m_{n}\right) \mid S_{n} / n>\delta m_{n}\right\} \\
\geq \frac{\tilde{E}_{n, \beta_{n}, K_{n}}\left\{\varphi\left(S_{n} / n^{1-\kappa}+W_{n} / n^{1 / 2-\kappa}-n^{\kappa} m_{n}\right) \mid A_{n}\left(\delta_{2}\right)\right\}-\tilde{\Gamma}_{n, 1}}{\tilde{\Gamma}_{n, 2}+\tilde{\Gamma}_{n, 3}} .
\end{gathered}
$$

(b) We have the conditioned limit

$$
\begin{aligned}
\lim _{n \rightarrow \infty} & \tilde{E}_{n, \beta_{n}, K_{n}}\left\{f\left(S_{n} / n^{1-\kappa}+W_{n} / n^{1 / 2-\kappa}-n^{\kappa} m_{n}\right) \mid S_{n} / n>\delta m_{n}\right\} \\
= & \lim _{n \rightarrow \infty} \tilde{E}_{n, \beta_{n}, K_{n}}\left\{f\left(S_{n} / n^{1-\kappa}+W_{n} / n^{1 / 2-\kappa}-n^{\kappa} m_{n}\right) \mid A_{n}(\delta)\right\} \\
= & \frac{1}{\int_{\mathbb{R}} \exp \left[-\frac{1}{2} g^{(2)}(\bar{x}) x^{2}\right] d x} \cdot \int_{\mathbb{R}} f(x) \exp \left[-\frac{1}{2} g^{(2)}(\bar{x}) x^{2}\right] d x .
\end{aligned}
$$

Proof of part (a) of Lemma 10.4. The hypotheses of this lemma are a subset of the hypotheses of Lemma 8.9 and 10.3. We start by proving the first inequality in part (a). By the first inequality in Lemma 10.3 and the second inequality in part (a) of Lemma 8.9 we have for all sufficiently large $n$

$$
\begin{aligned}
& \tilde{E}_{n, \beta_{n}, K_{n}}\left\{\varphi\left(S_{n} / n^{1-\kappa}+W_{n} / n^{1 / 2-\kappa}-n^{\kappa} m_{n}\right) \mid S_{n} / n>\delta m_{n}\right\} \\
& =\frac{\tilde{E}_{n, \beta_{n}, K_{n}}\left\{\varphi\left(S_{n} / n^{1-\kappa}+W_{n} / n^{1 / 2-\kappa}-n^{\kappa} m_{n}\right) \cdot 1_{\left\{S_{n} / n>\delta m_{n}\right\}}\right\}}{P_{n, \beta_{n}, K_{n}}\left\{S_{n} / n>\delta m_{n}\right\}} \\
& \leq \frac{\tilde{E}_{n, \beta_{n}, K_{n}}\left\{\varphi\left(S_{n} / n^{1-\kappa}+W_{n} / n^{1 / 2-\kappa}-n^{\kappa} m_{n}\right) \cdot 1_{A_{n}\left(\delta_{1}\right)}\right\}+\|\varphi\|_{\infty} e^{-c n^{2 \zeta}}}{\left(P_{n, \beta_{n}, K_{n}} \times Q\right)\left\{A_{n}\left(\delta_{2}\right)\right\}-e^{-c n^{2 \zeta}}} \\
& =\frac{\frac{\tilde{E}_{n, \beta_{n}, K_{n}}\left\{\varphi\left(S_{n} / n^{1-\kappa}+W_{n} / n^{1 / 2-\kappa}-n^{\kappa} m_{n}\right) \cdot 1_{\left.A_{n}\left(\delta_{1}\right)\right\}}\right.}{\left(P_{n, \beta_{n}, K_{n}} \times Q\right)\left\{A_{n}\left(\delta_{1}\right)\right\}}+\frac{\|\varphi\|_{\infty} e^{-c n^{2 \zeta}}}{\left(P_{n, \beta_{n}, K_{n}} \times Q\right)\left\{A_{n}\left(\delta_{1}\right)\right\}}}{\frac{\left(P_{n, \beta_{n}, K_{n}} \times Q\right)\left\{A_{n}\left(\delta_{2}\right)\right\}}{\left(P_{n, \beta_{n}, K_{n}} \times Q\right)\left\{A_{n}\left(\delta_{1}\right)\right\}}-\frac{e^{-c n^{2 \zeta}}}{\left(P_{n, \beta_{n}, K_{n}} \times Q\right)\left\{A_{n}\left(\delta_{1}\right)\right\}}} \\
& =\frac{\tilde{E}_{n, \beta_{n}, K_{n}}\left\{\varphi\left(S_{n} / n^{1-\kappa}+W_{n} / n^{1 / 2-\kappa}-n^{\kappa} m_{n}\right) \mid A_{n}\left(\delta_{1}\right)\right\}+\tilde{\Theta}_{n, 1}}{\tilde{\Theta}_{n, 2}-\tilde{\Theta}_{n, 3}},
\end{aligned}
$$


where $c>0$ is constant and

$$
\begin{gathered}
\tilde{\Theta}_{n, 1}=\frac{\|\varphi\|_{\infty} e^{-c n^{2 \zeta}}}{\left(P_{n, \beta_{n}, K_{n}} \times Q\right)\left\{A_{n}\left(\delta_{1}\right)\right\}}, \\
\tilde{\Theta}_{n, 2}=\frac{\left(P_{n, \beta_{n}, K_{n}} \times Q\right)\left\{A_{n}\left(\delta_{2}\right)\right\}}{\left(P_{n, \beta_{n}, K_{n}} \times Q\right)\left\{A_{n}\left(\delta_{1}\right)\right\}},
\end{gathered}
$$

and

$$
\tilde{\Theta}_{n, 3}=\frac{e^{-c n^{2 \zeta}}}{\left(P_{n, \beta_{n}, K_{n}} \times Q\right)\left\{A_{n}\left(\delta_{1}\right)\right\}} .
$$

We prove the first inequality in part (a) of the present lemma by showing that as $n \rightarrow \infty$, $\tilde{\Theta}_{n, 1} \rightarrow 0, \tilde{\Theta}_{n, 2} \rightarrow 1$ and $\tilde{\Theta}_{n, 3} \rightarrow 0$. These limits hold for any $0<\delta_{1}<1$ and $0<\delta_{2}<1$. By (8.2) in part (a) of Lemma 8.2 with $\bar{\delta}=\delta_{1}$

$$
\begin{aligned}
\tilde{\Theta}_{n, 1} & =\frac{\|\varphi\|_{\infty} e^{-c n^{2 \zeta}}}{\left(P_{n, \beta_{n}, K_{n}} \times Q\right)\left\{A_{n}\left(\delta_{1}\right)\right\}} \\
& =\frac{\|\varphi\|_{\infty} e^{-c n^{2 \zeta}}}{\int_{-n^{\kappa}\left(1-\delta_{1}\right) m_{n}}^{\infty} \exp \left[-n G_{\beta_{n}, K_{n}}\left(x / n^{\kappa}+m_{n}\right)\right] d x / Z_{n, \kappa}} \\
& =\frac{\exp \left[n G_{\beta_{n}, K_{n}}\left(m_{n}\right)\right] \cdot Z_{n, \kappa} \cdot\|\varphi\|_{\infty} e^{-c n^{2 \zeta}}}{\int_{-n^{\kappa}\left(1-\delta_{1}\right) m_{n}}^{\infty} \exp \left[-n G_{\beta_{n}, K_{n}}\left(x / n^{\kappa}+m_{n}\right)+n G_{\beta_{n}, K_{n}}\left(m_{n}\right)\right] d x}
\end{aligned}
$$

We now use Lemma 8.7 for $f \equiv 1$ and $\bar{\delta}=\delta_{1}$. This gives

$\lim _{n \rightarrow \infty} \int_{-n^{\kappa}\left(1-\delta_{1}\right) m_{n}}^{\infty} \exp \left[-n G_{\beta_{n}, K_{n}}\left(x / n^{\kappa}+m_{n}\right)+n G_{\beta_{n}, K_{n}}\left(m_{n}\right)\right] d x=\int_{\mathbb{R}} \exp \left[-\frac{1}{2} g^{(2)}(\bar{x}) x^{2}\right] d x$

By Lemma 8.4 for any $\varepsilon>0$ and all sufficiently large $n$

$$
\exp \left[n G_{\beta_{n}, K_{n}}\left(m_{n}\right)\right] \cdot Z_{n, \kappa} \cdot\|\varphi\|_{\infty} e^{-c n^{2 \zeta}} \leq\|\varphi\|_{\infty} \exp \left[\varepsilon n^{1-\alpha / \alpha_{0}}-c n^{2 \zeta}\right]
$$

Since by hypothesis $\zeta>\frac{1}{2}\left(1-\alpha / \alpha_{0}\right)$, it follows that

$$
\lim _{n \rightarrow \infty} \exp \left[n G_{\beta_{n}, K_{n}}\left(m_{n}\right)\right] \cdot Z_{n, \kappa} \cdot\|\varphi\|_{\infty} e^{-c n^{2 \zeta}}=0 .
$$


It follows from the last line of (10.9) that

$$
\begin{aligned}
\lim _{n \rightarrow \infty} \tilde{\Theta}_{n, 1} & =\lim _{n \rightarrow \infty} \frac{\exp \left[n G_{\beta_{n}, K_{n}}\left(m_{n}\right)\right] \cdot Z_{n, \kappa} \cdot\|\varphi\|_{\infty} e^{-c n^{2 \zeta}}}{\int_{-n^{\kappa}\left(1-\delta_{1}\right) m_{n}}^{\infty} \exp \left[-n G_{\beta_{n}, K_{n}}\left(x / n^{\kappa}+m_{n}\right)+n G_{\beta_{n}, K_{n}}\left(m_{n}\right)\right] d x} \\
& =\frac{0}{\int_{\mathbb{R}} \exp \left[-\frac{1}{2} g^{(2)}(\bar{x}) x^{2}\right] d x}=0,
\end{aligned}
$$

as claimed.

We now prove that $\lim _{n \rightarrow \infty} \tilde{\Theta}_{n, 2}=0$. By (8.2) in part (a) of Lemma 8.2 with $\bar{\delta}=\delta_{1}$ and $\bar{\delta}=\delta_{2}$

$$
\begin{aligned}
\tilde{\Theta}_{n, 2} & =\frac{\left(P_{n, \beta_{n}, K_{n}} \times Q\right)\left\{A_{n}\left(\delta_{2}\right)\right\}}{\left(P_{n, \beta_{n}, K_{n}} \times Q\right)\left\{A_{n}\left(\delta_{1}\right)\right\}} \\
& =\frac{\int_{-n^{\kappa}\left(1-\delta_{2}\right) m_{n}}^{\infty} \exp \left[-n G_{\beta_{n}, K_{n}}\left(x / n^{\kappa}+m_{n}\right)\right] d x / Z_{n, \kappa}}{\int_{-n^{\kappa}\left(1-\delta_{1}\right) m_{n}}^{\infty} \exp \left[-n G_{\beta_{n}, K_{n}}\left(x / n^{\kappa}+m_{n}\right)\right] d x / Z_{n, \kappa}} \\
& =\frac{\int_{-n^{\kappa}\left(1-\delta_{2}\right) m_{n}}^{\infty} \exp \left[-n G_{\beta_{n}, K_{n}}\left(x / n^{\kappa}+m_{n}\right)+n G_{\beta_{n}, K_{n}}\left(m_{n}\right)\right] d x}{\int_{-n^{\kappa}\left(1-\delta_{1}\right) m_{n}}^{\infty} \exp \left[-n G_{\beta_{n}, K_{n}}\left(x / n^{\kappa}+m_{n}\right)+n G \beta_{n}, K_{n}\left(m_{n}\right)\right] d x} .
\end{aligned}
$$

By Lemma 8.7 for $f=1, \bar{\delta}=\delta_{1}$, and $\bar{\delta}=\delta_{2}$, both the numerator and denominator have the same limit

$$
\int_{\mathbb{R}} \exp \left[-\frac{1}{2} g^{(2)}(\bar{x}) x^{2}\right] d x
$$

It follows that $\lim _{n \rightarrow \infty} \tilde{\Theta}_{n, 2}=1$ as claimed.

We now prove that $\lim _{n \rightarrow \infty} \tilde{\Theta}_{n, 3}=0$. The limit $\lim _{n \rightarrow \infty} \tilde{\Theta}_{n, 3}=0$ is a special case of the $\operatorname{limit} \lim _{n \rightarrow \infty} \tilde{\Theta}_{n, 1}=0$ with $\phi \equiv 1$. This completes the proof of the first inequality in part (a) of the present lemma.

We now prove the second inequality in part (a) of the present lemma. By the second inequality in Lemma 10.3 and the first inequality in part (a) of Lemma 8.9 we have for all sufficiently large $n$ 


$$
\begin{aligned}
& \tilde{E}_{n, \beta_{n}, K_{n}}\left\{\varphi\left(S_{n} / n^{1-\kappa}+W_{n} / n^{1 / 2-\kappa}-n^{\kappa} m_{n}\right) \mid S_{n} / n>\delta m_{n}\right\} \\
& =\frac{\tilde{E}_{n, \beta_{n}, K_{n}}\left\{\varphi\left(S_{n} / n^{1-\kappa}+W_{n} / n^{1 / 2-\kappa}-n^{\kappa} m_{n}\right) \cdot 1_{\left\{S_{n} / n>\delta m_{n}\right\}}\right\}}{P_{n, \beta_{n}, K_{n}}\left\{S_{n} / n>\delta m_{n}\right\}} \\
& \geq \frac{\tilde{E}_{n, \beta_{n}, K_{n}}\left\{\varphi\left(S_{n} / n^{1-\kappa}+W_{n} / n^{1 / 2-\kappa}-n^{\kappa} m_{n}\right) \cdot 1_{A_{n}\left(\delta_{2}\right)}\right\}-\|\varphi\|_{\infty} e^{-c n^{2 \zeta}}}{\left(P_{n, \beta_{n}, K_{n}} \times Q\right)\left\{A_{n}\left(\delta_{1}\right)\right\}+e^{-c n^{2 \zeta}}} \\
& =\frac{\frac{\tilde{E}_{n, \beta_{n}, K_{n}}\left\{\varphi\left(S_{n} / n^{1-\kappa}+W_{n} / n^{1 / 2-\kappa}-n^{\kappa} m_{n}\right) \cdot 1_{A_{n}\left(\delta_{2}\right)}\right\}}{\left(P_{n, \beta_{n}, K_{n}} \times Q\right)\left\{A_{n}\left(\delta_{2}\right)\right\}}-\frac{\|\varphi\|_{\infty} e^{-c n^{2 \zeta}}}{\left(P_{n, \beta_{n}, K_{n}} \times Q\right)\left\{A_{n}\left(\delta_{2}\right)\right\}}}{\frac{\left(P_{n, \beta_{n}, K_{n}} \times Q\right)\left\{A_{n}\left(\delta_{1}\right)\right\}}{\left(P_{n, \beta_{n}, K_{n}} \times Q\right)\left\{A_{n}\left(\delta_{2}\right)\right\}}+\frac{e^{-c n^{2 \zeta}}}{\left(P_{n, \beta_{n}, K_{n}} \times Q\right)\left\{A_{n}\left(\delta_{2}\right)\right\}}} \\
& =\frac{\tilde{E}_{n, \beta_{n}, K_{n}}\left\{\varphi\left(S_{n} / n^{1-\kappa}+W_{n} / n^{1 / 2-\kappa}-n^{\kappa} m_{n}\right) \mid A_{n}\left(\delta_{2}\right)\right\}-\tilde{\Gamma}_{n, 1}}{\tilde{\Gamma}_{n, 2}+\tilde{\Gamma}_{n, 3}},
\end{aligned}
$$

where

$$
\begin{aligned}
\tilde{\Gamma}_{n, 1} & =\frac{\|\varphi\|_{\infty} e^{-c n^{2 \zeta}}}{\left(P_{n, \beta_{n}, K_{n}} \times Q\right)\left\{A_{n}\left(\delta_{2}\right)\right\}}, \\
\tilde{\Gamma}_{n, 2} & =\frac{\left(P_{n, \beta_{n}, K_{n}} \times Q\right)\left\{A_{n}\left(\delta_{1}\right)\right\}}{\left(P_{n, \beta_{n}, K_{n}} \times Q\right)\left\{A_{n}\left(\delta_{2}\right)\right\}},
\end{aligned}
$$

and

$$
\tilde{\Gamma}_{n, 3}=\frac{e^{-c n^{2 \zeta}}}{\left(P_{n, \beta_{n}, K_{n}} \times Q\right)\left\{A_{n}\left(\delta_{2}\right)\right\}} .
$$

The sequences $\tilde{\Gamma}_{n, 1}, \tilde{\Gamma}_{n, 2}$, and $\tilde{\Gamma}_{n, 3}$ are obtained from $\tilde{\Theta}_{n, 1}, \tilde{\Theta}_{n, 2}$, and $\tilde{\Theta}_{n, 3}$ in (10.6)-(10.8) by interchanging $\delta_{1}$ and $\delta_{2}$. Hence the limits $\tilde{\Gamma}_{n, 1} \rightarrow 0, \tilde{\Gamma}_{n, 2} \rightarrow 1$ and $\tilde{\Gamma}_{n, 3} \rightarrow 0$ follow from the limits $\tilde{\Theta}_{n, 1} \rightarrow 0, \tilde{\Theta}_{n, 2} \rightarrow 1$ and $\tilde{\Theta}_{n, 3} \rightarrow 0$, which hold for any $0<\delta_{1}<1$ and $0<\delta_{2}<1$. The proof of part (a) of Lemma 10.4 is complete.

Proof of part (b) of Lemma 10.4. We know from Lemma 10.1 that, as $n \rightarrow \infty$, for any $\bar{\delta}=\delta_{1}$ and $\bar{\delta}=\delta_{2}, \tilde{E}_{n, \beta_{n}, K_{n}}\left\{\varphi\left(S_{n} / n^{1-\kappa}+W_{n} / n^{1 / 2-\kappa}-n^{\kappa} m_{n}\right) \mid A_{n}(\bar{\delta})\right\}$ has the same limit

$$
\frac{1}{\int_{\mathbb{R}} \exp \left[-\frac{1}{2} g^{(2)}(\bar{x}) x^{2}\right] d x} \cdot \int_{\mathbb{R}} \varphi(x) \exp \left[-\frac{1}{2} g^{(2)}(\bar{x}) x^{2}\right] d x .
$$

By sending $n \rightarrow \infty$ in the inequality in part (a) of Lemma 10.4 we have 


$$
\begin{aligned}
& \frac{1}{\int_{\mathbb{R}} \exp \left[-\frac{1}{2} g^{(2)}(\bar{x}) x^{2}\right] d x} \cdot \int_{\mathbb{R}} \varphi(x) \exp \left[-\frac{1}{2} g^{(2)}(\bar{x}) x^{2}\right] d x \\
& =\lim _{n \rightarrow \infty} \tilde{E}_{n, \beta_{n}, K_{n}}\left\{\varphi\left(S_{n} / n^{1-\kappa}+W_{n} / n^{1 / 2-\kappa}-n^{\kappa} m_{n}\right) \mid A_{n}\left(\delta_{1}\right)\right\} \\
& \geq \limsup _{n \rightarrow \infty} \tilde{E}_{n, \beta_{n}, K_{n}}\left\{\varphi\left(S_{n} / n^{1-\kappa}+W_{n} / n^{1 / 2-\kappa}-n^{\kappa} m_{n}\right) \mid S_{n} / n>\delta m_{n}\right\} \\
& \geq \liminf _{n \rightarrow \infty} \tilde{E}_{n, \beta_{n}, K_{n}}\left\{\varphi\left(S_{n} / n^{1-\kappa}+W_{n} / n^{1 / 2-\kappa}-n^{\kappa} m_{n}\right) \mid S_{n} / n>\delta m_{n}\right\} \\
& =\lim _{n \rightarrow \infty} \tilde{E}_{n, \beta_{n}, K_{n}}\left\{\varphi\left(S_{n} / n^{1-\kappa}+W_{n} / n^{1 / 2-\kappa}-n^{\kappa} m_{n}\right) \mid A_{n}\left(\delta_{2}\right)\right\} \\
& =\frac{1}{\int_{\mathbb{R}} \exp \left[-\frac{1}{2} g^{(2)}(\bar{x}) x^{2}\right] d x} \cdot \int_{\mathbb{R}} \varphi(x) \exp \left[-\frac{1}{2} g^{(2)}(\bar{x}) x^{2}\right] d x .
\end{aligned}
$$

Because the first and last terms in this display are the same, it follows that

$$
\begin{gathered}
\lim _{n \rightarrow \infty} \tilde{E}_{n, \beta_{n}, K_{n}}\left\{\varphi\left(S_{n} / n^{1-\kappa}+W_{n} / n^{1 / 2-\kappa}-n^{\kappa} m_{n}\right) \mid S_{n} / n>\delta m_{n}\right\} \\
=\frac{1}{\int_{\mathbb{R}} \exp \left[-\frac{1}{2} g^{(2)}(\bar{x}) x^{2}\right] d x} \cdot \int_{\mathbb{R}} \varphi(x) \exp \left[-\frac{1}{2} g^{(2)}(\bar{x}) x^{2}\right] d x .
\end{gathered}
$$

Define $f^{+}=\max (f, 0)$ and $f^{-}=\max (-f, 0)$. Then $f=f^{+}-f^{-}$. Since $f^{+} \geq 0$ and $f^{-} \geq 0$ we apply (10.10) to $\varphi=f^{+}$and $\varphi=f^{-}$, obtaining

$$
\begin{gathered}
\lim _{n \rightarrow \infty} \tilde{E}_{n, \beta_{n}, K_{n}}\left\{f^{+}\left(S_{n} / n^{1-\kappa}+W_{n} / n^{1 / 2-\kappa}-n^{\kappa} m_{n}\right) \mid S_{n} / n>\delta m_{n}\right\} \\
=\frac{1}{\int_{\mathbb{R}} \exp \left[-\frac{1}{2} g^{(2)}(\bar{x}) x^{2}\right] d x} \cdot \int_{\mathbb{R}} f^{+}(x) \exp \left[-\frac{1}{2} g^{(2)}(\bar{x}) x^{2}\right] d x
\end{gathered}
$$

and

$$
\begin{gathered}
\lim _{n \rightarrow \infty} \tilde{E}_{n, \beta_{n}, K_{n}}\left\{f^{-}\left(S_{n} / n^{1-\kappa}+W_{n} / n^{1 / 2-\kappa}-n^{\kappa} m_{n}\right) \mid S_{n} / n>\delta m_{n}\right\} \\
=\frac{1}{\int_{\mathbb{R}} \exp \left[-\frac{1}{2} g^{(2)}(\bar{x}) x^{2}\right] d x} \cdot \int_{\mathbb{R}} f^{-}(x) \exp \left[-\frac{1}{2} g^{(2)}(\bar{x}) x^{2}\right] d x .
\end{gathered}
$$

Together the preceding two limits prove that

$$
\begin{gathered}
\lim _{n \rightarrow \infty} \tilde{E}_{n, \beta_{n}, K_{n}}\left\{f\left(S_{n} / n^{1-\kappa}+W_{n} / n^{1 / 2-\kappa}-n^{\kappa} m_{n}\right) \mid S_{n} / n>\delta m_{n}\right\} \\
=\frac{1}{\int_{\mathbb{R}} \exp \left[-\frac{1}{2} g^{(2)}(\bar{x}) x^{2}\right] d x} \cdot \int_{\mathbb{R}} f(x) \exp \left[-\frac{1}{2} g^{(2)}(\bar{x}) x^{2}\right] d x .
\end{gathered}
$$


On the other hand, by Lemma 10.1 with $\bar{\delta}=\delta$

$$
\begin{aligned}
\lim _{n \rightarrow \infty} \tilde{E}_{n, \beta_{n}, K_{n}}\left\{f\left(S_{n} / n^{1-\kappa}+W_{n} / n^{1 / 2-\kappa}-n^{\kappa} m_{n}\right) \mid A_{n}(\delta)\right\} \\
=\frac{1}{\int_{\mathbb{R}} \exp \left[-\frac{1}{2} g^{(2)}(\bar{x}) x^{2}\right] d x} \cdot \int_{\mathbb{R}} f(x) \exp \left[-\frac{1}{2} g^{(2)}(\bar{x}) x^{2}\right] d x
\end{aligned}
$$

The proof of part (b) of this lemma is complete.

We now put together the pieces to complete the proof of part (a) of Theorem 6.1. Let $f$ be any bounded, uniformly continuous function mapping $\mathbb{R}$ to $\mathbb{R}$ and let $\delta$ be any number satisfying $\Delta<\delta<1$, where $\Delta \in(0,1)$ is determined in part (b) of Lemma 8.5. The proof of part (a) of Theorem 6.1 is subdivided into Step 1, Substep 2a, and Substep 2b. Step 1 is done in Lemma 10.1. There we prove that with $\bar{\delta}=\delta$

$$
\begin{gathered}
\lim _{n \rightarrow \infty} \tilde{E}_{n, \beta_{n}, K_{n}}\left\{f\left(S_{n} / n^{1-\kappa}+W_{n} / n^{1 / 2-\kappa}-n^{\kappa} m_{n}\right) \mid S_{n} / n+W_{n} / n^{1 / 2}>\delta m_{n}\right\} \\
=\frac{1}{\int_{\mathbb{R}} \exp \left[-\frac{1}{2} g^{(2)}(\bar{x}) x^{2}\right] d x} \cdot \int_{\mathbb{R}} f(x) \exp \left[-\frac{1}{2} g^{(2)}(\bar{x}) x^{2}\right] d x .
\end{gathered}
$$

Substep $2 \mathrm{a}$ is done in Lemma 10.2. There we prove that

$$
\lim _{n \rightarrow \infty}\left|\tilde{C}_{n}-\tilde{D}_{n}\right|=0
$$

where

$$
\tilde{C}_{n}=E_{n, \beta_{n}, K_{n}}\left\{f\left(S_{n} / n^{1-\kappa}-n^{\kappa} m_{n}\right) \mid S_{n} / n>\delta m_{n}\right\}
$$

and

$$
\tilde{D}_{n}=\tilde{E}_{n, \beta_{n}, K_{n}}\left\{f\left(S_{n} / n^{1-\kappa}+W_{n} / n^{1 / 2-\kappa}-n^{\kappa} m_{n}\right) \mid S_{n} / n>\delta m_{n}\right\} .
$$

Substep $2 \mathrm{~b}$ is done in part (b) of Lemma 10.4. There we prove that

$$
\lim _{n \rightarrow \infty} \tilde{D}_{n}=\lim _{n \rightarrow \infty} \tilde{F}_{n}=\frac{1}{\int_{\mathbb{R}} \exp \left[-\frac{1}{2} g^{(2)}(\bar{x}) x^{2}\right] d x} \cdot \int_{\mathbb{R}} f(x) \exp \left[-\frac{1}{2} g^{(2)}(\bar{x}) x^{2}\right] d x
$$


where

$$
\tilde{F}_{n}=\tilde{E}_{n, \beta_{n}, K_{n}}\left\{f\left(S_{n} / n^{1-\kappa}+W_{n} / n^{1 / 2-\kappa}-n^{\kappa} m_{n}\right) \mid S_{n} / n+W_{n} / n^{1 / 2}>\delta m_{n}\right\} .
$$

Taking together these limits yield the limit

$$
\begin{aligned}
\lim _{n \rightarrow \infty} & E_{n, \beta_{n}, K_{n}}\left\{f\left(S_{n} / n^{1-\kappa}-n^{\kappa} m_{n}\right) \mid S_{n} / n>\delta m_{n}\right\} \\
= & \lim _{n \rightarrow \infty} \tilde{E}_{n, \beta_{n}, K_{n}}\left\{f\left(S_{n} / n^{1-\kappa}+W_{n} / n^{1 / 2-\kappa}-n^{\kappa} m_{n}\right) \mid S_{n} / n+W_{n} / n^{1 / 2}>\delta m_{n}\right\} \\
= & \frac{1}{\int_{\mathbb{R}} \exp \left[-\frac{1}{2} g^{(2)}(\bar{x}) x^{2}\right] d x} \cdot \int_{\mathbb{R}} f(x) \exp \left[-\frac{1}{2} g^{(2)}(\bar{x}) x^{2}\right] d x .
\end{aligned}
$$

This gives the weak limit stated in part (a) of Theorem 6.1:

$$
P_{n, \beta_{n}, K_{n}}\left\{n^{\kappa}\left(S_{n} / n-m\left(\beta_{n}, K_{n}\right)\right) \in d x \mid S_{n} / n>\delta m\left(\beta_{n}, K_{n}\right)\right\} \Longrightarrow N\left(0,1 / g^{(2)}(\bar{x})\right)
$$

The proof of part (a) of Theorem 6.1 is complete. 


\section{APPENDIX A \\ PROOF THAT SEQUENCES 1A-5A SATISFY THE LIMITS IN HYPOTHESIS (III') OF THEOREM 4.1}

In this appendix we prove that sequences 1a-5a satisfy the limits in hypothesis (iii') of Theorem 4.1. These limits take the following form.

(a) Assume that $g$ has degree 4. For $\forall \alpha \in\left(0, \alpha_{0}\right)$ and for $j=2,3,4$

$$
\lim _{n \rightarrow \infty} n^{\alpha / \alpha_{0}-j \theta \alpha} G_{\beta_{n}, K_{n}}^{(j)}\left(m\left(\beta_{n}, K_{n}\right)\right)=g^{(j)}(\bar{x})
$$

(b) Assume that $g$ has degree 6. For $\forall \alpha \in\left(0, \alpha_{0}\right)$ and for $j=2,3,4,5,6$

$$
\lim _{n \rightarrow \infty} n^{\alpha / \alpha_{0}-j \theta \alpha} G_{\beta_{n}, K_{n}}^{(j)}\left(m\left(\beta_{n}, K_{n}\right)\right)=g^{(j)}(\bar{x})
$$

We do this by verifying the limits (A.1) and (A.3) in Lemma A.1. Let $\varepsilon_{n}$ denote a sequence that converges to 0 and that represents the various error terms arising in the proof. We use the same notation $\varepsilon_{n}$ to represent different error terms.

Lemma A.1. We assume the hypotheses of Theorem 3.1. We also assume (A.1) when the degree of the Ginzburg-Landau polynomial $g$ is 4 and (A.3) when the degree of $g$ is 6 . 
(a) Assume that $g$ has degree 4 and that for $\alpha \in\left(0, \alpha_{0}\right)$ and for $j=2,3,4$

$$
\lim _{n \rightarrow \infty} n^{\alpha / \alpha_{0}-j \theta \alpha} G_{\beta_{n}, K_{n}}^{(j)}\left(x / n^{\theta \alpha}\right)=g^{(j)}(x)
$$

uniformly for $x$ in compact subsets of $\mathbb{R}$. Then we have

$$
\lim _{n \rightarrow \infty} n^{\alpha / \alpha_{0}-j \theta \alpha} G_{\beta_{n}, K_{n}}^{(j)}\left(m\left(\beta_{n}, K_{n}\right)\right)=g^{(j)}(\bar{x}) .
$$

(b) Assume that $g$ has degree 6 and that for $\alpha \in\left(0, \alpha_{0}\right)$ and for $j=2,3,4,5,6$

$$
\lim _{n \rightarrow \infty} n^{\alpha / \alpha_{0}-j \theta \alpha} G_{\beta_{n}, K_{n}}^{(j)}\left(x / n^{\theta \alpha}\right)=g^{(j)}(x)
$$

uniformly for $x$ in compact subsets of $\mathbb{R}$. Then we have

$$
\lim _{n \rightarrow \infty} n^{\alpha / \alpha_{0}-j \theta \alpha} G_{\beta_{n}, K_{n}}^{(j)}\left(m\left(\beta_{n}, K_{n}\right)\right)=g^{(j)}(\bar{x})
$$

Proof. We write $m_{n}=m\left(\beta_{n}, K_{n}\right)$. When $g$ has degree 4 , we have for $j=2,3,4$, and when $g$ has degree 6 , we have for $j=2,3,4,5,6$

$$
\begin{aligned}
\left|n^{\alpha / \alpha_{0}-j \theta \alpha} G_{\beta_{n} K_{n}}^{(j)}\left(m_{n}\right)-g^{(j)}(\bar{x})\right| \leq & \left|n^{\alpha / \alpha_{0}-j \theta \alpha} G_{\beta_{n} K_{n}}^{(j)}\left(n^{\theta \alpha} m_{n} / n^{\theta \alpha}\right)-g^{(j)}\left(n^{\theta \alpha} m_{n}\right)\right| \\
& +\left|g^{(j)}\left(n^{\theta \alpha} m_{n}\right)-g^{(j)}(\bar{x})\right| .
\end{aligned}
$$

Let $\Xi$ be any compact subset of $\mathbb{R}$. By hypothesis (A.1) for $j=2,3,4$ when $g$ has degree 4 and by hypothesis (A.3) for $j=2,3,4,5,6$ when $g$ has degree 6

$$
\lim _{n \rightarrow \infty} \sup _{x \in \Xi}\left|n^{\alpha / \alpha_{0}-j \theta \alpha} G_{\beta_{n}, K_{n}}^{(j)}\left(x / n^{\theta \alpha}\right)-g^{(j)}(x)\right|=0 .
$$

According to Theorem 3.1, $n^{\theta \alpha} m_{n} \rightarrow \bar{x}$, and so for any $\varepsilon>0$ the sequence $n^{\theta \alpha} m_{n}$ lies in the compact set $[\bar{x}-\varepsilon, \bar{x}+\varepsilon]$ for all sufficiently large $n$. It follows that the first term on the 
right-hand side of (A.5) converges to 0 as $n \rightarrow \infty$. Because of the limit $n^{\theta \alpha} m_{n} \rightarrow \bar{x}$ and the continuity of $g^{(j)}$, the second term on the right-hand side of (A.5) also converges to 0 as $n \rightarrow \infty$. We conclude that for $j=2,3,4$ when $g$ has degree 4 and for $j=2,3,4,5,6$ when $g$ has degree 6

$$
\left|n^{\alpha / \alpha_{0}-j \theta \alpha} G_{\beta_{n}, K_{n}}^{(j)}\left(m_{n}\right)-g^{(j)}(\bar{x})\right| \rightarrow 0 \text { as } n \rightarrow \infty .
$$

This completes the proof of the lemma.

The main point of this section is to justify rigorously the limits in (A.1) and (A.3) for sequences $1 \mathrm{a}-5 \mathrm{a}$. We start by doing some preparatory work involving the Taylor expansion of $G_{\beta_{n}, K_{n}}^{(j)}\left(x / n^{\gamma}\right)$ for $\gamma>0$.

Case 1: $g$ has degree $4, j=2,3,4$. This case arises for sequences 1 and 2 , which converge to a second-order point $(\beta, K(\beta))$ for $0<\beta<\beta_{c}$. We consider the Taylor expansions of $G_{\beta_{n}, K_{n}}^{(j)}\left(x / n^{\gamma}\right)$ to order 4 with error terms. Since $K(\beta)=(4 \beta+2) / 4 \beta$ is continuous and $\left(\beta_{n}, K_{n}\right)$ converges to $(\beta, K(\beta))$, we have $\beta_{n} K_{n} / K\left(\beta_{n}\right) \rightarrow \beta$. Thus the coefficients in Taylor expansion of $G_{\beta_{n}, K_{n}}^{(j)}\left(x / n^{\gamma}\right)$ are given by

$$
\begin{gathered}
G_{\beta_{n}, K_{n}}^{(2)}(0)=2 \beta_{n} K_{n}-\frac{8 \beta_{n}^{2} K_{n}^{2}}{e^{\beta_{n}}+2}=\frac{2 \beta_{n} K_{n}\left(K\left(\beta_{n}\right)-K_{n}\right)}{K\left(\beta_{n}\right)}=2 \beta\left(K\left(\beta_{n}\right)-K_{n}\right)\left(1+\varepsilon_{n}\right), \\
G_{\beta_{n}, K_{n}}^{(3)}(0)=0, \\
G_{\beta_{n}, K_{n}}^{(4)}(0)=\frac{2\left(2 \beta_{n} K_{n}\right)^{4}\left(4-e^{\beta_{n}}\right)}{\left(e^{\beta_{n}}+2\right)^{2}} .
\end{gathered}
$$

Let $c_{4}(\beta)=\left(e^{\beta}+2\right)^{2}\left(4-e^{\beta}\right) /(8 \cdot 4 !)$. Since $2 \beta_{n} K_{n} \rightarrow 2 \beta K(\beta)=\left(e^{\beta}+2\right) / 2$, we have

$$
G_{\beta_{n}, K_{n}}^{(4)}(0)=\left(e^{\beta}+2\right)^{2}\left(4-e^{\beta}\right)\left(1+\varepsilon_{n}\right) / 8=c_{4}(\beta)\left(1+\varepsilon_{n}\right) \cdot 4 !
$$


Thus for all $n \in \mathbb{N}$, any $\gamma>0$, any $R>0$, and all $x \in \mathbb{R}$ satisfying $\left|x / n^{\gamma}\right|<R$, we have the Taylor expansion

$$
G_{\beta_{n}, K_{n}}^{(2)}\left(x / n^{\gamma}\right)=G_{\beta_{n}, K_{n}}^{(2)}(0)+\frac{G_{\beta_{n}, K_{n}}^{(4)}(0)}{2 !} \cdot \frac{x^{2}}{n^{2 \gamma}}+\mathrm{O}\left(\frac{1}{n^{3 \gamma}}\right) x^{3}
$$

Multiplying both sides by $n^{1-u-2 \gamma}$ for $u>0$ yields

$$
\begin{aligned}
n^{1-u-2 \gamma} G_{\beta_{n}, K_{n}}^{(2)}\left(x / n^{\gamma}\right)= & \frac{1}{n^{2 \gamma-1+u}} G_{\beta_{n}, K_{n}}^{(2)}(0)+\frac{1}{n^{4 \gamma-1+u}} \cdot \frac{G_{\beta_{n}, K_{n}}^{(4)}(0)}{2 !} x^{2} \\
& +\mathrm{O}\left(\frac{1}{n^{5 \gamma-1+u}}\right) x^{3} \\
= & \frac{1}{n^{2 \gamma-1+u}} \cdot 2 \beta\left(K\left(\beta_{n}\right)-K_{n}\right)\left(1+\varepsilon_{n}\right) \\
& +\frac{1}{n^{4 \gamma-1+u}} \cdot \frac{c_{4}(\beta)\left(1+\varepsilon_{n}\right) \cdot 4 !}{2 !} x^{2}+\mathrm{O}\left(\frac{1}{n^{5 \gamma-1+u}}\right) x^{3} .
\end{aligned}
$$

For all $n \in \mathbb{N}$, any $\gamma>0$, any $R>0$, and all $x \in \mathbb{R}$ satisfying $\left|x / n^{\gamma}\right|<R$, we have the Taylor expansion

$$
G_{\beta_{n}, K_{n}}^{(3)}\left(x / n^{\gamma}\right)=G_{\beta_{n}, K_{n}}^{(4)}(0) \cdot \frac{x}{n^{\gamma}}+\mathrm{O}\left(\frac{1}{n^{2 \gamma}}\right) x^{2}
$$

Multiplying both sides by $n^{1-u-3 \gamma}$ for $u>0$ yields

$$
\begin{aligned}
n^{1-u-3 \gamma} G_{\beta_{n}, K_{n}}^{(3)}\left(x / n^{\gamma}\right) & =\frac{1}{n^{4 \gamma-1+u}} \cdot G_{\beta_{n}, K_{n}}^{(4)}(0) \cdot x+\mathrm{O}\left(\frac{1}{n^{5 \gamma-1+u}}\right) x^{2} \\
& =\frac{1}{n^{4 \gamma-1+u}} \cdot c_{4}(\beta)\left(1+\varepsilon_{n}\right) \cdot 4 ! \cdot x+\mathrm{O}\left(\frac{1}{n^{5 \gamma-1+u}}\right) x^{2}
\end{aligned}
$$

For all $n \in \mathbb{N}$, any $\gamma>0$, any $R>0$, and all $x \in \mathbb{R}$ satisfying $\left|x / n^{\gamma}\right|<R$, we have the Taylor expansion

$$
G_{\beta_{n}, K_{n}}^{(4)}\left(x / n^{\gamma}\right)=G_{\beta_{n}, K_{n}}^{(4)}(0)+\mathrm{O}\left(\frac{1}{n^{\gamma}}\right) x
$$


Multiplying both sides by $n^{1-u-4 \gamma}$ for $u>0$ yields

$$
\begin{aligned}
n^{1-u-4 \gamma} G_{\beta_{n}, K_{n}}^{(4)}\left(x / n^{\gamma}\right) & =\frac{1}{n^{4 \gamma-1+u}} \cdot G_{\beta_{n}, K_{n}}^{(4)}(0)+\mathrm{O}\left(\frac{1}{n^{5 \gamma-1+u}}\right) x \\
& =\frac{1}{n^{4 \gamma-1+u}} \cdot c_{4}(\beta)\left(1+\varepsilon_{n}\right) \cdot 4 !+\mathrm{O}\left(\frac{1}{n^{5 \gamma-1+u}}\right) x .
\end{aligned}
$$

In formulas (A.6)-(A.8) the big-oh terms are uniform for $x \in\left(-R n^{\gamma}, R n^{\gamma}\right)$. We will use (A.6)-(A.8) to verify hypothesis (A.1) for sequences 1a and 2a.

Case 2: $g$ has degree $6, j=2,3,4,5,6$. This case arises for sequences 3,4 and 5, which converge to the tricritical point $\left(\beta_{c}, K\left(\beta_{c}\right)\right)$. We consider the Taylor expansions of $G_{\beta_{n}, K_{n}}^{(j)}\left(x / n^{\gamma}\right)$ to order 6 with error terms. Since $K(\beta)=(4 \beta+2) / 4 \beta$ is continuous and $\left(\beta_{n}, K_{n}\right)$ converges to $\left(\beta_{c}, K\left(\beta_{c}\right)\right)$, we have $\beta_{n} K_{n} / K\left(\beta_{n}\right) \rightarrow \beta_{c}$. Thus the coefficient in Taylor expansion of $G_{\beta_{n}, K_{n}}^{(j)}\left(x / n^{\gamma}\right)$ are given by

$$
\begin{gathered}
G_{\beta_{n}, K_{n}}^{(2)}(0)=2 \beta_{n} K_{n}-\frac{8 \beta_{n}^{2} K_{n}^{2}}{e^{\beta_{n}}+2}=\frac{2 \beta_{n} K_{n}\left(K\left(\beta_{n}\right)-K_{n}\right)}{K\left(\beta_{n}\right)}=2 \beta_{c}\left(K\left(\beta_{n}\right)-K_{n}\right)\left(1+\varepsilon_{n}\right), \\
G_{\beta_{n}, K_{n}}^{(3)}(0)=0, \\
G_{\beta_{n}, K_{n}}^{(4)}(0)=\frac{2\left(2 \beta_{n} K_{n}\right)^{4}\left(4-e^{\beta_{n}}\right)}{\left(e^{\beta_{n}}+2\right)^{2}} .
\end{gathered}
$$

Let $c_{4}=3 / 16$. Since $2 \beta_{n} K_{n} \rightarrow 2 \beta_{c} K\left(\beta_{c}\right)=\left(e^{\beta_{c}}+2\right) / 2=3$ and $e^{\beta_{n}}+2 \rightarrow e^{\beta_{c}}+2=6$, we have

$$
\begin{gathered}
G_{\beta_{n}, K_{n}}^{(4)}(0)=2 \cdot 3^{4}\left(4-e^{\beta_{n}}\right)\left(1+\varepsilon_{n}\right) / 6^{2}=c_{4}\left(4-e^{\beta_{n}}\right)\left(1+\varepsilon_{n}\right) \cdot 4 ! \\
G_{\beta_{n}, K_{n}}^{(5)}(0)=0 .
\end{gathered}
$$

Let $c_{6}=9 / 40$, since $G_{\beta_{n}, K_{n}}^{(6)}(0) \rightarrow G_{\beta_{c}, K\left(\beta_{c}\right)}^{(6)}(0)=2 \cdot 3^{4}$, we have

$$
G_{\beta_{n}, K_{n}}^{(6)}(0)=2 \cdot 3^{4}\left(1+\varepsilon_{n}\right)=c_{6}\left(1+\varepsilon_{n}\right) \cdot 6 !
$$


Thus for all $n \in \mathbb{N}$, any $\gamma>0$, any $R>0$, and all $x \in \mathbb{R}$ satisfying $\left|x / n^{\gamma}\right|<R$, we have the Taylor expansion

$$
G_{\beta_{n}, K_{n}}^{(2)}\left(x / n^{\gamma}\right)=G_{\beta_{n}, K_{n}}^{(2)}(0)+\frac{G_{\beta_{n}, K_{n}}^{(4)}(0)}{2 !} \cdot \frac{x^{2}}{n^{2 \gamma}}+\frac{G_{\beta_{n}, K_{n}}^{(6)}(0)}{4 !} \cdot \frac{x^{4}}{n^{4 \gamma}}+\mathrm{O}\left(\frac{1}{n^{5 \gamma}}\right) x^{5}
$$

Multiplying both sides by $n^{1-u-2 \gamma}$ for $u>0$ yields

$$
\begin{aligned}
n^{1-u-2 \gamma} G_{\beta_{n}, K_{n}}^{(2)}\left(x / n^{\gamma}\right)= & \frac{1}{n^{2 \gamma-1+u}} G_{\beta_{n}, K_{n}}^{(2)}(0)+\frac{1}{n^{4 \gamma-1+u}} \cdot \frac{G_{\beta_{n}, K_{n}}^{(4)}(0)}{2 !} x^{2} \\
& +\frac{1}{n^{6 \gamma-1+u}} \cdot \frac{G_{\beta_{n}, K_{n}}^{(6)}(0)}{4 !} x^{4}+\mathrm{O}\left(\frac{1}{n^{7 \gamma-1+u}}\right) x^{5} \\
= & \frac{1}{n^{2 \gamma-1+u}} \cdot 2 \beta_{c}\left(K\left(\beta_{n}\right)-K_{n}\right)\left(1+\varepsilon_{n}\right) \\
& +\frac{1}{n^{4 \gamma-1+u}} \cdot \frac{c_{4}\left(4-e^{\beta_{n}}\right)\left(1+\varepsilon_{n}\right) \cdot 4 !}{2 !} x^{2}+\frac{c_{6}\left(1+\varepsilon_{n}\right) \cdot 6 !}{4 !} x^{4} \\
& +\mathrm{O}\left(\frac{1}{n^{7 \gamma-1+u}}\right) x^{5} .
\end{aligned}
$$

For all $n \in \mathbb{N}$, any $\gamma>0$, any $R>0$, and all $x \in \mathbb{R}$ satisfying $\left|x / n^{\gamma}\right|<R$, we have the Taylor expansion

$$
G_{\beta_{n}, K_{n}}^{(3)}\left(x / n^{\gamma}\right)=G_{\beta_{n}, K_{n}}^{(4)}(0) \cdot \frac{x}{n^{\gamma}}+\frac{G_{\beta_{n}, K_{n}}^{(6)}(0)}{3 !} \cdot \frac{x^{3}}{n^{3 \gamma}}+\mathrm{O}\left(\frac{1}{n^{4 \gamma}}\right) x^{4}
$$

Multiplying both sides by $n^{1-u-3 \gamma}$ for $u>0$ yields

$$
\begin{aligned}
n^{1-u-3 \gamma} G_{\beta_{n}, K_{n}}^{(3)}\left(x / n^{\gamma}\right)= & \frac{1}{n^{4 \gamma-1+u}} \cdot G_{\beta_{n}, K_{n}}^{(4)}(0) \cdot x+\frac{1}{n^{6 \gamma-1+u}} \cdot \frac{G_{\beta_{n}, K_{n}}^{(6)}(0)}{3 !} \cdot x^{3} \\
& +\mathrm{O}\left(\frac{1}{n^{7 \gamma-1+u}}\right) x^{4} \\
= & \frac{1}{n^{4 \gamma-1+u}} \cdot c_{4}\left(4-e^{\beta_{n}}\right)\left(1+\varepsilon_{n}\right) \cdot 4 ! \cdot x+\frac{1}{n^{6 \gamma-1+u}} \cdot \frac{c_{6}\left(1+\varepsilon_{n}\right) \cdot 6 !}{3 !} x^{3} \\
& +\mathrm{O}\left(\frac{1}{n^{7 \gamma-1+u}}\right) x^{4} .
\end{aligned}
$$


For all $n \in \mathbb{N}$, any $\gamma>0$, any $R>0$, and all $x \in \mathbb{R}$ satisfying $\left|x / n^{\gamma}\right|<R$, we have the Taylor expansion

$$
G_{\beta_{n}, K_{n}}^{(4)}\left(x / n^{\gamma}\right)=G_{\beta_{n}, K_{n}}^{(4)}(0)+\frac{G_{\beta_{n}, K_{n}}^{(6)}(0)}{2 !} \cdot \frac{x^{2}}{n^{2 \gamma}}+\mathrm{O}\left(\frac{1}{n^{3 \gamma}}\right) x^{3}
$$

Multiplying both sides by $n^{1-u-4 \gamma}$ for $u>0$ yields

$$
\begin{aligned}
n^{1-u-4 \gamma} G_{\beta_{n}, K_{n}}^{(4)}\left(x / n^{\gamma}\right)= & \frac{1}{n^{4 \gamma-1+u}} \cdot G_{\beta_{n}, K_{n}}^{(4)}(0)+\frac{1}{n^{6 \gamma-1+u}} \cdot \frac{G_{\beta_{n}, K_{n}}^{(6)}(0)}{2 !} \cdot x^{2} \\
& +\mathrm{O}\left(\frac{1}{n^{7 \gamma-1+u}}\right) x^{3} \\
= & \frac{1}{n^{4 \gamma-1+u}} \cdot c_{4}\left(4-e^{\beta_{n}}\right)\left(1+\varepsilon_{n}\right) \cdot 4 !+\frac{1}{n^{6 \gamma-1+u}} \cdot \frac{c_{6}\left(1+\varepsilon_{n}\right) \cdot 6 !}{2 !} \cdot x^{2} \\
& +\mathrm{O}\left(\frac{1}{n^{7 \gamma-1+u}}\right) x^{3} .
\end{aligned}
$$

For all $n \in \mathbb{N}$, any $\gamma>0$, any $R>0$, and all $x \in \mathbb{R}$ satisfying $\left|x / n^{\gamma}\right|<R$, we have the Taylor expansion

$$
G_{\beta_{n}, K_{n}}^{(5)}\left(x / n^{\gamma}\right)=G_{\beta_{n}, K_{n}}^{(6)}(0) \cdot \frac{x}{n^{\gamma}}+\mathrm{O}\left(\frac{1}{n^{2 \gamma}}\right) x^{2}
$$

Multiplying both sides by $n^{1-u-5 \gamma}$ for $u>0$ yields

$$
\begin{aligned}
n^{1-u-5 \gamma} G_{\beta_{n}, K_{n}}^{(5)}\left(x / n^{\gamma}\right) & =\frac{1}{n^{6 \gamma-1+u}} \cdot G_{\beta_{n}, K_{n}}^{(6)}(0) x+\mathrm{O}\left(\frac{1}{n^{7 \gamma-1+u}}\right) x^{2} \\
& =\frac{1}{n^{6 \gamma-1+u}} \cdot c_{6}\left(1+\varepsilon_{n}\right) \cdot 6 ! \cdot x+\mathrm{O}\left(\frac{1}{n^{7 \gamma-1+u}}\right) x^{2}
\end{aligned}
$$

For all $n \in \mathbb{N}$, any $\gamma>0$, any $R>0$, and all $x \in \mathbb{R}$ satisfying $\left|x / n^{\gamma}\right|<R$, we have the Taylor expansion

$$
G_{\beta_{n}, K_{n}}^{(6)}\left(x / n^{\gamma}\right)=G_{\beta_{n}, K_{n}}^{(6)}(0)+\mathrm{O}\left(\frac{1}{n^{\gamma}}\right) x
$$


Multiplying both sides by $n^{1-u-6 \gamma}$ for $u>0$ yields

$$
\begin{aligned}
n^{1-u-6 \gamma} G_{\beta_{n}, K_{n}}^{(6)}\left(x / n^{\gamma}\right) & =\frac{1}{n^{6 \gamma-1+u}} \cdot G_{\beta_{n}, K_{n}}^{(6)}(0)+\mathrm{O}\left(\frac{1}{n^{7 \gamma-1+u}}\right) x \\
& =\frac{1}{n^{6 \gamma-1+u}} \cdot c_{6}\left(1+\varepsilon_{n}\right) \cdot 6 ! \cdot+\mathrm{O}\left(\frac{1}{n^{7 \gamma-1+u}}\right) x
\end{aligned}
$$

In formulas (A.9)-(A.13) the big-oh terms are uniform for $x \in\left(-R n^{\gamma}, R n^{\gamma}\right)$. We will use (A.9)-(A.13) to verify assumption (A.3) for sequences 3a, 4a, 5a.

\section{Sequence 1a}

This sequence is defined in (5.5). For sequence 1a, $g$ has degree 4. Since $K\left(\beta_{n}\right)-K_{n}=$ $\left(K^{\prime}(\beta) b-k\right) / n^{\alpha}+\mathrm{O}\left(1 / n^{2 \alpha}\right)$, it follows from (A.6) that for all $n \in \mathbb{N}$, any $u>0$, any $\gamma>0$, any $R>0$, and all $x \in \mathbb{R}$ satisfying $\left|x / n^{\gamma}\right|<R$, we have

$$
\begin{aligned}
n^{1-u-2 \gamma} G_{\beta_{n}, K_{n}}^{(2)}\left(x / n^{\gamma}\right)= & \frac{1}{n^{2 \gamma-1+u+\alpha}} 2 \beta\left(K^{\prime}(\beta) b-k\right)\left(1+\varepsilon_{n}\right) \\
& +\frac{1}{n^{4 \gamma-1+u}} \cdot \frac{c_{4}(\beta)\left(1+\varepsilon_{n}\right) \cdot 4 !}{2 !} x^{2} \\
& +\mathrm{O}\left(\frac{1}{n^{2 \gamma-1+u+2 \alpha}}\right)+\mathrm{O}\left(\frac{1}{n^{5 \gamma-1+u}}\right) x^{3} .
\end{aligned}
$$

We now define $\gamma=\theta \alpha$ and $u=1-\alpha / \alpha_{0}$, and we recall that $\alpha_{0}=1 / 2, \theta=1 / 2$. With these choices of $\gamma$ and $u$, the powers of $n$ appearing in the first two terms in (A.14) are 0 , and the powers of $n$ appearing in the last two terms in (A.14) are positive. Letting $n \rightarrow \infty$ in (A.14), we have uniformly for $x$ in compact subsets of $\mathbb{R}$

$$
\begin{aligned}
\lim _{n \rightarrow \infty} n^{1-u-2 \gamma} G_{\beta_{n}, K_{n}}^{(2)}\left(x / n^{\gamma}\right) & =\lim _{n \rightarrow \infty} n^{\alpha / \alpha_{0}-2 \theta \alpha} G_{\beta_{n}, K_{n}}^{(2)}\left(x / n^{\gamma}\right) \\
& =2 \beta\left(K^{\prime}(\beta) b-k\right)+\frac{c_{4}(\beta) \cdot 4 !}{2 !} x^{2}=g^{(2)}(x) .
\end{aligned}
$$

The same choices of $\gamma$ and $u$ ensure that the powers of $n$ appearing in the first term in (A.7) and (A.8) are 0, and the powers of $n$ appearing in the last term in (A.7) and (A.8) are 
positive. Taking $n \rightarrow \infty$ in (A.7) and (A.8) gives

$$
\begin{aligned}
\lim _{n \rightarrow \infty} n^{1-u-3 \gamma} G_{\beta_{n}, K_{n}}^{(3)}\left(x / n^{\gamma}\right) & =\lim _{n \rightarrow \infty} n^{\alpha / \alpha_{0}-3 \theta \alpha} G_{\beta_{n}, K_{n}}^{(3)}\left(x / n^{\gamma}\right) \\
& =c_{4}(\beta) \cdot 4 ! \cdot x=g^{(3)}(x) .
\end{aligned}
$$

and

$$
\begin{aligned}
\lim _{n \rightarrow \infty} n^{1-u-4 \gamma} G_{\beta_{n}, K_{n}}^{(4)}\left(x / n^{\gamma}\right) & =\lim _{n \rightarrow \infty} n^{\alpha / \alpha_{0}-4 \theta \alpha} G_{\beta_{n}, K_{n}}^{(4)}\left(x / n^{\gamma}\right) \\
& =c_{4}(\beta) \cdot 4 !=g^{(4)}(x) .
\end{aligned}
$$

uniformly for $x$ in compact subsets of $\mathbb{R}$. Thus sequence 1 satisfies hypothesis (A.1) in Lemma A.1, and so the conclusion (A.2) in Lemma A.1 follows for $j=2,3,4$. This is the convergence in hypothesis (iii') of Theorem 4.1.

\section{Sequence 2a}

This sequence is defined in (5.6). For sequence $2 \mathrm{a}, g$ has degree 4. Since $K\left(\beta_{n}\right)-K_{n}=$ $\left(K^{(p)}(\beta)-\ell\right) b^{p} / p ! n^{p \alpha}+\mathrm{O}\left(1 / n^{\alpha(p+1)}\right)$, it follows from (A.6) that for all $n \in \mathbb{N}$, any $u>0$, any $\gamma>0$, any $R>0$, and all $x \in \mathbb{R}$ satisfying $\left|x / n^{\gamma}\right|<R$, we have

$$
\begin{aligned}
n^{1-u-2 \gamma} G_{\beta_{n}, K_{n}}^{(2)}\left(x / n^{\gamma}\right)= & \frac{1}{n^{2 \gamma-1+u+\alpha}} \cdot \frac{1}{p !} \cdot 2 \beta\left(K^{(p)}(\beta)-\ell\right) b^{p}\left(1+\varepsilon_{n}\right) \\
& +\frac{1}{n^{4 \gamma-1+u}} \cdot \frac{c_{4}(\beta)\left(1+\varepsilon_{n}\right) \cdot 4 !}{2 !} x^{2} \\
& +\mathrm{O}\left(\frac{1}{n^{2 \gamma-1+u+(p+1) \alpha}}\right)+\mathrm{O}\left(\frac{1}{n^{5 \gamma-1+u}}\right) x^{3} .
\end{aligned}
$$

We now define $\gamma=\theta \alpha$ and $u=1-\alpha / \alpha_{0}$, and we recall that $\alpha_{0}=1 / 2 p, \theta=p / 2$. With these choices of $\gamma$ and $u$, the power of $n$ appearing in the first two terms in (A.15) are 0 , 
and the power of $n$ appearing in the last two terms in (A.15) are positive. Letting $n \rightarrow \infty$ in (A.15), we have uniformly for $x$ in compact subsets of $\mathbb{R}$

$$
\begin{aligned}
\lim _{n \rightarrow \infty} n^{1-u-2 \gamma} G_{\beta_{n}, K_{n}}^{(2)}\left(x / n^{\gamma}\right) & =\lim _{n \rightarrow \infty} n^{\alpha / \alpha_{0}-2 \theta \alpha} G_{\beta_{n}, K_{n}}^{(2)}\left(x / n^{\gamma}\right) \\
& =\frac{1}{p !} \cdot 2 \beta\left(K^{(p)}(\beta)-\ell\right) b^{p}+\frac{c_{4}(\beta) \cdot 4 !}{2 !} x^{2}=g^{(2)}(x)
\end{aligned}
$$

The same choices of $\gamma$ and $u$ ensure that the powers of $n$ appearing in the first term in (A.7) and (A.8) are 0, and the powers of $n$ appearing in the last term in (A.7) and (A.8) are positive. Taking $n \rightarrow \infty$ in (A.7) and (A.8) gives

$$
\begin{aligned}
\lim _{n \rightarrow \infty} n^{1-u-3 \gamma} G_{\beta_{n}, K_{n}}^{(3)}\left(x / n^{\gamma}\right) & =\lim _{n \rightarrow \infty} n^{\alpha / \alpha_{0}-3 \theta \alpha} G_{\beta_{n}, K_{n}}^{(3)}\left(x / n^{\gamma}\right) \\
& =c_{4}(\beta) \cdot 4 ! \cdot x=g^{(3)}(x)
\end{aligned}
$$

and

$$
\begin{aligned}
\lim _{n \rightarrow \infty} n^{1-u-4 \gamma} G_{\beta_{n}, K_{n}}^{(4)}\left(x / n^{\gamma}\right) & =\lim _{n \rightarrow \infty} n^{\alpha / \alpha_{0}-4 \theta \alpha} G_{\beta_{n}, K_{n}}^{(4)}\left(x / n^{\gamma}\right) \\
& =c_{4}(\beta) \cdot 4 !=g^{(4)}(x) .
\end{aligned}
$$

uniformly for $x$ in compact subsets of $\mathbb{R}$. Thus sequence 2 satisfies hypothesis (A.1) in Lemma A.1, and so the conclusion (A.2) in Lemma A.1 follows for $j=2,3,4$. This is the convergence in hypothesis (iii') of Theorem 4.1.

\section{Sequence 3a}

This sequence is defined in (5.7). For sequence $3 \mathrm{a}, g$ has degree 6. Since $K\left(\beta_{n}\right)-K_{n}=$ $\left(K^{\prime}\left(\beta_{c}\right) b-k\right) / n^{\alpha}+\mathrm{O}\left(1 / n^{2 \alpha}\right)$, it follows (A.9), (A.10), and (A.11) that for all $n \in \mathbb{N}$, any $u>0$, any $\gamma>0$, any $R>0$, and all $x \in \mathbb{R}$ satisfying $\left|x / n^{\gamma}\right|<R$, we have the following: 


$$
\begin{aligned}
n^{1-u-2 \gamma} G_{\beta_{n}, K_{n}}^{(2)}\left(x / n^{\gamma}\right)= & \frac{1}{n^{2 \gamma-1+u+\alpha}} \cdot 2 \beta_{c}\left(K^{\prime}\left(\beta_{c}\right) b-k\right)\left(1+\varepsilon_{n}\right) \\
& +\frac{1}{n^{4 \gamma-1+u+\alpha}} \cdot \frac{c_{4}(-4 b)\left(1+\varepsilon_{n}\right) \cdot 4 !}{2 !} x^{2} \\
& +\frac{1}{n^{6 \gamma-1+u}} \cdot \frac{c_{6}\left(1+\varepsilon_{n}\right) \cdot 6 !}{4 !} \cdot x^{4}+\mathrm{O}\left(\frac{1}{n^{2 \gamma-1+u+2 \alpha}}\right) \\
+ & \mathrm{O}\left(\frac{1}{n^{4 \gamma-1+u+2 \alpha}}\right) x^{2}+\mathrm{O}\left(\frac{1}{n^{7 \gamma-1+u}}\right) x^{5} \\
n^{1-u-3 \gamma} G_{\beta_{n}, K_{n}}^{(3)}\left(x / n^{\gamma}\right)= & \frac{1}{n^{4 \gamma-1+u+\alpha} \cdot c_{4}(-4 b)\left(1+\varepsilon_{n}\right) \cdot 4 ! \cdot x} \\
& +\frac{1}{n^{6 \gamma-1+u}} \cdot \frac{c_{6}\left(1+\varepsilon_{n}\right) \cdot 6 !}{3 !} \cdot x^{3} \\
& +\mathrm{O}\left(\frac{1}{n^{4 \gamma-1+u+2 \alpha}}\right) x+\mathrm{O}\left(\frac{1}{n^{7 \gamma-1+u}}\right) x^{4}
\end{aligned}
$$

and

$$
\begin{aligned}
n^{1-u-4 \gamma} G_{\beta_{n}, K_{n}}^{(4)}\left(x / n^{\gamma}\right)= & \frac{1}{n^{4 \gamma-1+u+\alpha}} \cdot c_{4}(-4 b)\left(1+\varepsilon_{n}\right) \cdot 4 ! \\
& +\frac{1}{n^{6 \gamma-1+u}} \cdot \frac{c_{6}\left(1+\varepsilon_{n}\right) \cdot 6 !}{2 !} \cdot x^{2} \\
& +\mathrm{O}\left(\frac{1}{n^{4 \gamma-1+u+2 \alpha}}\right) x+\mathrm{O}\left(\frac{1}{n^{7 \gamma-1+u}}\right) x^{3} .
\end{aligned}
$$

We now define $\gamma=\theta \alpha$ and $u=1-\alpha / \alpha_{0}$, and we recall that $\alpha_{0}=2 / 3, \theta=1 / 4$. With these choices of $\gamma$ and $u$, the powers of $n$ appearing in the first term and the third term in (A.16) are 0 , and the powers of $n$ appearing in the second term and the last three terms in (A.16) are positive. Letting $n \rightarrow \infty$ in (A.16), we have uniformly for $x$ in compact subsets of $\mathbb{R}$

$$
\begin{aligned}
\lim _{n \rightarrow \infty} n^{1-u-2 \gamma} G_{\beta_{n}, K_{n}}^{(2)}\left(x / n^{\gamma}\right) & =\lim _{n \rightarrow \infty} n^{\alpha / \alpha_{0}-2 \theta \alpha} G_{\beta_{n}, K_{n}}^{(2)}\left(x / n^{\gamma}\right) \\
& =2 \beta_{c}\left(K^{\prime}\left(\beta_{c}\right) b-k\right)+\frac{c_{6} \cdot 6 !}{4 !} x^{4}=g^{(2)}(x)
\end{aligned}
$$


The same choices of $\gamma$ and $u$ ensure that the powers of $n$ appearing in the second term in (A.17) and (A.18) are 0, and the powers of $n$ appearing in the first term and last two terms in (A.17) and (A.18) are positive. Taking $n \rightarrow \infty$ in (A.17) and (A.18) gives that

$$
\begin{aligned}
\lim _{n \rightarrow \infty} n^{1-u-3 \gamma} G_{\beta_{n}, K_{n}}^{(3)}\left(x / n^{\gamma}\right) & =\lim _{n \rightarrow \infty} n^{\alpha / \alpha_{0}-3 \theta \alpha} G_{\beta_{n}, K_{n}}^{(3)}\left(x / n^{\gamma}\right) \\
& =\frac{c_{6} \cdot 6 !}{3 !} x^{3}=g^{(3)}(x)
\end{aligned}
$$

and

$$
\begin{aligned}
\lim _{n \rightarrow \infty} n^{1-u-4 \gamma} G_{\beta_{n}, K_{n}}^{(4)}\left(x / n^{\gamma}\right) & =\lim _{n \rightarrow \infty} n^{\alpha / \alpha_{0}-4 \theta \alpha} G_{\beta_{n}, K_{n}}^{(4)}\left(x / n^{\gamma}\right) \\
& =\frac{c_{6} \cdot 6 !}{2 !} x^{2}=g^{(4)}(x) .
\end{aligned}
$$

uniformly for $x$ in compact subsets of $\mathbb{R}$. Similarly, the powers of $n$ appearing in the first term in the expansions (A.12) and (A.13) are 0, and the powers of $n$ appearing in the last term in the expansions (A.12) and (A.13) are positive. Letting $n \rightarrow \infty$ in (A.12) and (A.13), we have uniformly for $x$ in compact subsets of $\mathbb{R}$

$$
\begin{aligned}
\lim _{n \rightarrow \infty} n^{1-u-5 \gamma} G_{\beta_{n}, K_{n}}^{(5)}\left(x / n^{\gamma}\right) & =\lim _{n \rightarrow \infty} n^{\alpha / \alpha_{0}-5 \theta \alpha} G_{\beta_{n}, K_{n}}^{(5)}\left(x / n^{\gamma}\right) \\
& =c_{6} \cdot 6 ! x=g^{(5)}(x)
\end{aligned}
$$

and

$$
\begin{aligned}
\lim _{n \rightarrow \infty} n^{1-u-6 \gamma} G_{\beta_{n}, K_{n}}^{(6)}\left(x / n^{\gamma}\right) & =\lim _{n \rightarrow \infty} n^{\alpha / \alpha_{0}-6 \theta \alpha} G_{\beta_{n}, K_{n}}^{(6)}\left(x / n^{\gamma}\right) \\
& =c_{6} \cdot 6 !=g^{(6)}(x) .
\end{aligned}
$$

Thus sequence 3 satisfies hypothesis (A.3) in Lemma A.1, and so the conclusion (A.4) in Lemma A.1 follows for $j=2,3,4,5,6$. This is the convergence in hypothesis (iii') of Theorem 4.1. 


\section{Sequence 4a}

This sequence is defined in (5.8). For sequence $4 \mathrm{a}, g$ has degree 6. Since

$$
\begin{aligned}
K\left(\beta_{n}\right)-K_{n}= & K\left(\beta_{c}+1 / n^{\alpha}\right)-K_{n} \\
= & K\left(\beta_{c}\right)+K^{\prime}\left(\beta_{c}\right) \cdot 1 / n^{\alpha}+K^{\prime \prime}\left(\beta_{c}\right) \cdot 1 / 2 ! n^{2 \alpha}+K^{\prime \prime \prime}\left(\beta_{c}\right) \cdot 1 / 3 ! n^{3 \alpha} \\
& +\mathrm{O}\left(1 / n^{4 \alpha}\right)-K_{n} \\
= & \left(K^{\prime \prime}\left(\beta_{c}\right)-\ell\right) / 2 n^{2 \alpha}+\left(K^{\prime \prime \prime}\left(\beta_{c}\right)-\tilde{\ell}\right) / 6 n^{3 \alpha}+\mathrm{O}\left(1 / n^{4 \alpha}\right)
\end{aligned}
$$

and

$$
4-e^{\beta_{n}}=-4 / n^{\alpha}+\mathrm{O}\left(1 / n^{2 \alpha}\right),
$$

it follows from (A.9), (A.10), and (A.11) that for all $n \in \mathbb{N}$, any $u>0$, any $\gamma>0$, any $R>0$, and all $x \in \mathbb{R}$ satisfying $\left|x / n^{\gamma}\right|<R$, we have the following:

$$
\begin{aligned}
& n^{1-u-2 \gamma} G_{\beta_{n}, K_{n}}^{(2)}\left(x / n^{\gamma}\right)=\frac{1}{n^{2 \gamma-1+u+2 \alpha}} \cdot 2 \beta_{c} \cdot\left(K^{\prime \prime}\left(\beta_{c}\right)-\ell\right) / 2 \cdot\left(1+\varepsilon_{n}\right) \\
& +\frac{1}{n^{4 \gamma-1+u+\alpha}} \cdot \frac{c_{4}(-4)\left(1+\varepsilon_{n}\right) \cdot 4 !}{2 !} x^{2} \\
& +\frac{1}{n^{6 \gamma-1+u}} \cdot \frac{c_{6}\left(1+\varepsilon_{n}\right) \cdot 6 !}{4 !} \cdot x^{4} \\
& +\mathrm{O}\left(\frac{1}{n^{2 \gamma-1+u+3 \alpha}}\right)+\mathrm{O}\left(\frac{1}{n^{2 \gamma-1+u+4 \alpha}}\right) \\
& +\mathrm{O}\left(\frac{1}{n^{4 \gamma-1+u+2 \alpha}}\right) x^{2}+\mathrm{O}\left(\frac{1}{n^{7 \gamma-1+u}}\right) x^{5}, \\
& n^{1-u-3 \gamma} G_{\beta_{n}, K_{n}}^{(3)}\left(x / n^{\gamma}\right)=\frac{1}{n^{4 \gamma-1+u+\alpha}} \cdot c_{4}(-4)\left(1+\varepsilon_{n}\right) \cdot 4 ! \cdot x \\
& +\frac{1}{n^{6 \gamma-1+u}} \cdot \frac{c_{6}\left(1+\varepsilon_{n}\right) \cdot 6 !}{3 !} \cdot x^{3} \\
& +\mathrm{O}\left(\frac{1}{n^{4 \gamma-1+u+2 \alpha}}\right) x+\mathrm{O}\left(\frac{1}{n^{7 \gamma-1+u}}\right) x^{4},
\end{aligned}
$$

and 


$$
\begin{aligned}
n^{1-u-4 \gamma} G_{\beta_{n}, K_{n}}^{(4)}\left(x / n^{\gamma}\right)= & \frac{1}{n^{4 \gamma-1+u+\alpha}} \cdot c_{4}(-4)\left(1+\varepsilon_{n}\right) \cdot 4 ! \\
& +\frac{1}{n^{6 \gamma-1+u}} \cdot \frac{c_{6}\left(1+\varepsilon_{n}\right) \cdot 6 !}{2 !} \cdot x^{2} \\
& +\mathrm{O}\left(\frac{1}{n^{4 \gamma-1+u+2 \alpha}}\right)+\mathrm{O}\left(\frac{1}{n^{7 \gamma-1+u}}\right) x^{3} .
\end{aligned}
$$

We now define $\gamma=\theta \alpha$ and $u=1-\alpha / \alpha_{0}$, and we recall that $\alpha_{0}=1 / 3, \theta=1 / 2$. With these choices of $\gamma$ and $u$, the powers of $n$ appearing in the first three terms in (A.19) are 0 , and the powers of $n$ appearing in the last four terms in (A.19) are positive. Letting $n \rightarrow \infty$ in (A.19), we have uniformly for $x$ in compact subsets of $\mathbb{R}$

$$
\begin{aligned}
\lim _{n \rightarrow \infty} n^{1-u-2 \gamma} G_{\beta_{n}, K_{n}}^{(2)}\left(x / n^{\gamma}\right) & =\lim _{n \rightarrow \infty} n^{\alpha / \alpha_{0}-2 \theta \alpha} G_{\beta_{n}, K_{n}}^{(2)}\left(x / n^{\gamma}\right) \\
& =2 \beta_{c}\left(K^{\prime \prime}\left(\beta_{c}\right)-\ell\right) / 2+\frac{c_{4}(-4) \cdot 4 !}{2 !} x^{2}+\frac{c_{6} \cdot 6 !}{4 !} x^{4}=g^{(2)}(x) .
\end{aligned}
$$

The same choices of $\gamma$ and $u$ ensure that the powers of $n$ appearing in the first two terms in (A.20) and (A.21) are 0 and the powers of $n$ appearing in the last two terms in (A.20) and (A.21) are positive. Taking $n \rightarrow \infty$ in (A.20) and (A.21) gives

$$
\begin{aligned}
\lim _{n \rightarrow \infty} n^{1-u-3 \gamma} G_{\beta_{n}, K_{n}}^{(3)}\left(x / n^{\gamma}\right) & =\lim _{n \rightarrow \infty} n^{\alpha / \alpha_{0}-3 \theta \alpha} G_{\beta_{n}, K_{n}}^{(3)}\left(x / n^{\gamma}\right) \\
& =c_{4}(-4) \cdot 4 ! x+\frac{c_{6} \cdot 6 !}{3 !} x^{3}=g^{(3)}(x)
\end{aligned}
$$

and

$$
\begin{aligned}
\lim _{n \rightarrow \infty} n^{1-u-4 \gamma} G_{\beta_{n}, K_{n}}^{(4)}\left(x / n^{\gamma}\right) & =\lim _{n \rightarrow \infty} n^{\alpha / \alpha_{0}-4 \theta \alpha} G_{\beta_{n}, K_{n}}^{(4)}\left(x / n^{\gamma}\right) \\
& =c_{4}(-4) \cdot 4 !+\frac{c_{6} \cdot 6 !}{2 !} x^{2}=g^{(4)}(x)
\end{aligned}
$$

uniformly for $x$ in compact subsets of $\mathbb{R}$. Similarly, the powers of $n$ appearing in the first term in the expansions (A.12) and (A.13) are 0 and the powers of $n$ appearing in the last 
term in the expansions (A.12) and (A.13) are positive. Letting $n \rightarrow \infty$ in (A.12) and (A.13), we have uniformly for $x$ in compact subsets of $\mathbb{R}$

$$
\begin{aligned}
\lim _{n \rightarrow \infty} n^{1-u-5 \gamma} G_{\beta_{n}, K_{n}}^{(5)}\left(x / n^{\gamma}\right) & =\lim _{n \rightarrow \infty} n^{\alpha / \alpha_{0}-5 \theta \alpha} G_{\beta_{n}, K_{n}}^{(5)}\left(x / n^{\gamma}\right) \\
& =c_{6} \cdot 6 ! x=g^{(5)}(x)
\end{aligned}
$$

and

$$
\begin{aligned}
\lim _{n \rightarrow \infty} n^{1-u-6 \gamma} G_{\beta_{n}, K_{n}}^{(6)}\left(x / n^{\gamma}\right) & =\lim _{n \rightarrow \infty} n^{\alpha / \alpha_{0}-6 \theta \alpha} G_{\beta_{n}, K_{n}}^{(6)}\left(x / n^{\gamma}\right) \\
& =c_{6} \cdot 6 !=g^{(6)}(x) .
\end{aligned}
$$

Thus sequence 4 satisfies hypothesis (A.3) in Lemma A.1, and so the conclusion (A.4) in Lemma A.1 follows for $j=2,3,4,5,6$. This is the convergence in hypothesis (iii') of Theorem 4.1.

\section{Sequence 5a}

This sequence is defined in (5.10). For sequence 5a, $g$ has degree 6. Since $K\left(\beta_{n}\right)-K_{n}=$ $\left(K^{\prime \prime}\left(\beta_{c}\right)-\ell\right) / 2 n^{2 \alpha}+\mathrm{O}\left(1 / n^{3 \alpha}\right)$ and $4-e^{\beta_{n}}=4 / n^{\alpha}+\mathrm{O}\left(1 / n^{2 \alpha}\right)$, it follows from (A.9), (A.10), and (A.11) that for all $n \in \mathbb{N}$, any $u>0$, any $\gamma>0$, any $R>0$, and all $x \in \mathbb{R}$ satisfying $\left|x / n^{\gamma}\right|<R$, we have the following:

$$
\begin{aligned}
n^{1-u-2 \gamma} G_{\beta_{n}, K_{n}}^{(2)}\left(x / n^{\gamma}\right)= & \frac{1}{n^{2 \gamma-1+u+2 \alpha}} \cdot 2 \beta_{c} \cdot\left(K^{\prime \prime}\left(\beta_{c}\right)-\ell\right) / 2 \cdot\left(1+\varepsilon_{n}\right) \\
& +\frac{1}{n^{4 \gamma-1+u+\alpha}} \cdot \frac{c_{4} \cdot 4 \cdot\left(1+\varepsilon_{n}\right) \cdot 4 !}{2 !} x^{2} \\
& +\frac{1}{n^{6 \gamma-1+u}} \cdot \frac{c_{6}\left(1+\varepsilon_{n}\right) \cdot 6 !}{4 !} \cdot x^{4} \\
& +\mathrm{O}\left(\frac{1}{n^{2 \gamma-1+u+3 \alpha}}\right) \\
& +\mathrm{O}\left(\frac{1}{n^{4 \gamma-1+u+2 \alpha}}\right) x^{2}+\mathrm{O}\left(\frac{1}{n^{7 \gamma-1+u}}\right) x^{5}
\end{aligned}
$$




$$
\begin{aligned}
n^{1-u-3 \gamma} G_{\beta_{n}, K_{n}}^{(3)}\left(x / n^{\gamma}\right)= & \frac{1}{n^{4 \gamma-1+u+\alpha}} \cdot c_{4} \cdot 4 \cdot\left(1+\varepsilon_{n}\right) \cdot 4 ! \cdot x \\
& +\frac{1}{n^{6 \gamma-1+u}} \cdot \frac{c_{6}\left(1+\varepsilon_{n}\right) \cdot 6 !}{3 !} \cdot x^{3} \\
& +\mathrm{O}\left(\frac{1}{n^{4 \gamma-1+u+2 \alpha}}\right) x+\mathrm{O}\left(\frac{1}{n^{7 \gamma-1+u}}\right) x^{4},
\end{aligned}
$$

and

$$
\begin{aligned}
n^{1-u-4 \gamma} G_{\beta_{n}, K_{n}}^{(4)}\left(x / n^{\gamma}\right)= & \frac{1}{n^{4 \gamma-1+u+\alpha}} \cdot c_{4} \cdot 4 \cdot\left(1+\varepsilon_{n}\right) \cdot 4 ! \\
& +\frac{1}{n^{6 \gamma-1+u}} \cdot \frac{c_{6}\left(1+\varepsilon_{n}\right) \cdot 6 !}{2 !} \cdot x^{2} \\
& +\mathrm{O}\left(\frac{1}{n^{4 \gamma-1+u+2 \alpha}}\right)+\mathrm{O}\left(\frac{1}{n^{7 \gamma-1+u}}\right) x^{3}
\end{aligned}
$$

We now define $\gamma=\theta \alpha$ and $u=1-\alpha / \alpha_{0}$, and we recall that $\alpha_{0}=1 / 3, \theta=1 / 2$. With these choices of $\gamma$ and $u$, the powers of $n$ appearing in the first three terms in (A.22) are 0 , and the powers of $n$ appearing in the last three terms in (A.22) are positive. Letting $n \rightarrow \infty$ in (A.22), we have uniformly for $x$ in compact subsets of $\mathbb{R}$

$$
\begin{aligned}
\lim _{n \rightarrow \infty} n^{1-u-2 \gamma} G_{\beta_{n}, K_{n}}^{(2)}\left(x / n^{\gamma}\right) & =\lim _{n \rightarrow \infty} n^{\alpha / \alpha_{0}-2 \theta \alpha} G_{\beta_{n}, K_{n}}^{(2)}\left(x / n^{\gamma}\right) \\
& =\beta_{c}\left(K^{\prime \prime}\left(\beta_{c}\right)-\ell\right) / 2+\frac{c_{4} \cdot 4 \cdot 4 !}{2 !} x^{2}+\frac{c_{6} \cdot 6 !}{4 !} x^{4}=g^{(2)}(x) .
\end{aligned}
$$

The same choices of $\gamma$ and $u$ ensure that the powers of $n$ appearing in the first two terms in (A.23) and (A.24) are 0 and the powers of $n$ appearing in the last two terms in (A.23) and (A.24) are positive. Taking $n \rightarrow \infty$ in (A.23) and (A.24) gives

$$
\begin{aligned}
\lim _{n \rightarrow \infty} n^{1-u-3 \gamma} G_{\beta_{n}, K_{n}}^{(3)}\left(x / n^{\gamma}\right) & =\lim _{n \rightarrow \infty} n^{\alpha / \alpha_{0}-3 \theta \alpha} G_{\beta_{n}, K_{n}}^{(3)}\left(x / n^{\gamma}\right) \\
& =c_{4} \cdot 4 \cdot 4 ! x+\frac{c_{6} \cdot 6 !}{3 !} x^{3}=g^{(3)}(x)
\end{aligned}
$$

and 


$$
\begin{aligned}
\lim _{n \rightarrow \infty} n^{1-u-4 \gamma} G_{\beta_{n}, K_{n}}^{(4)}\left(x / n^{\gamma}\right) & =\lim _{n \rightarrow \infty} n^{\alpha / \alpha_{0}-4 \theta \alpha} G_{\beta_{n}, K_{n}}^{(4)}\left(x / n^{\gamma}\right) \\
& =c_{4} \cdot 4 \cdot 4 !+\frac{c_{6} \cdot 6 !}{2 !} x^{2}=g^{(4)}(x)
\end{aligned}
$$

uniformly for $x$ in compact subsets of $\mathbb{R}$. Similarly, the powers of $n$ appearing in the first term in the expansions (A.12) and (A.13) are 0, and the powers of $n$ appearing in the last term in the expansions (A.12) and (A.13) are positive. Letting $n \rightarrow \infty$ in (A.12) and (A.13), we have uniformly for $x$ in compact subsets of $\mathbb{R}$

$$
\begin{aligned}
\lim _{n \rightarrow \infty} n^{1-u-5 \gamma} G_{\beta_{n}, K_{n}}^{(5)}\left(x / n^{\gamma}\right) & =\lim _{n \rightarrow \infty} n^{\alpha / \alpha_{0}-5 \theta \alpha} G_{\beta_{n}, K_{n}}^{(5)}\left(x / n^{\gamma}\right) \\
& =c_{6} \cdot 6 ! x=g^{(5)}(x)
\end{aligned}
$$

and

$$
\begin{aligned}
\lim _{n \rightarrow \infty} n^{1-u-6 \gamma} G_{\beta_{n}, K_{n}}^{(6)}\left(x / n^{\gamma}\right) & =\lim _{n \rightarrow \infty} n^{\alpha / \alpha_{0}-6 \theta \alpha} G_{\beta_{n}, K_{n}}^{(6)}\left(x / n^{\gamma}\right) \\
& =c_{6} \cdot 6 !=g^{(6)}(x) .
\end{aligned}
$$

Thus sequence 5 satisfies hypothesis (A.3) in Lemma A.1, and so the conclusion (A.4) in Lemma A.1 follows for $j=2,3,4,5,6$. This is the convergence in hypothesis (iii') of Theorem 4.1. 


\section{APPENDIX B PROOF OF THE MDP IN PART (A) OF THEOREM 6.2}

In this appendix we give the proof of the MDP stated in part (a) of Theorem 6.2. We restate the theorem here for easy reference. Concerning the proof of part (b) of Theorem 6.2, see the comment before Lemma 6.3.

Theorem 6.2. Let $\left(\beta_{n}, K_{n}\right)$ be a positive sequence converging either to a second-order point $(\beta, K(\beta)), 0<\beta<\beta_{c}$, or to the tricritical point $\left(\beta_{c}, K\left(\beta_{c}\right)\right)$. We assume that $\left(\beta_{n}, K_{n}\right)$ satisfies the hypotheses of Theorem 4.1 for all $0<\alpha<\alpha_{0}$. The following conclusions hold.

(a) For all $0<\alpha<\alpha_{0}, S_{n} / n^{1-\theta \alpha}$ satisfies the MDP with respect to $P_{n, \beta_{n}, K_{n}}$ with exponential speed $n^{1-\alpha / \alpha_{0}}$ and rate function $\Gamma(x)=g(x)-\inf _{y \in \mathbb{R}} g(y)$; in symbols

$$
P_{n, \beta_{n}, K_{n}}\left\{S_{n} / n^{1-\theta \alpha} \in d x\right\} \asymp \exp \left[-n^{1-\alpha / \alpha_{0}} \Gamma(x)\right] d x
$$

(b) The hypotheses of this theorem are satisfied by sequence 1a-5a defined in Table 5.1.

We work with an arbitrary $\alpha$ satisfying $0<\alpha<\alpha_{0}$. To ease the notation we write $\gamma=\theta \alpha$ and $u=1-\alpha / \alpha_{0}$. The hypotheses of Theorem 6.2 coincide with the hypotheses of Theorem 4.1, which in turn consist of hypothesis (iii') and the hypotheses of Theorem 3.1 for all $0<\alpha<\alpha_{0}$. Clearly we have $0<u=1-\alpha / \alpha_{0}<1$ and by hypothesis (iii') $0<\gamma=\theta \alpha<\theta \alpha_{0}<1 / 2$. In addition, $1-2 \gamma-u=\left(1-2 \theta \alpha_{0}\right) \alpha / \alpha_{0}>0$, which implies $1-2 \gamma>u$. 
The proof of Theorem 6.2 is analogous to the proof of Theorem 8.1 in [8]. Let $W_{n}$ be a sequence of normal random variables with mean 0 and variance $\sigma_{n}^{2}=\left(2 \beta_{n} K_{n}\right)^{-1}$ defined on a probability space $(\Omega, \mathcal{F}, Q)$. Theorem 6.2 is proved in two steps.

Step 1. $W_{n} / n^{1 / 2-\gamma}$ is superexponentially small relative to $\exp \left(n^{u}\right)$; i.e., for any $\delta>0$

$$
\limsup _{n \rightarrow \infty} \frac{1}{n^{u}} \log Q\left\{\left|W_{n} / n^{1 / 2-\gamma}\right|>\delta\right\}=-\infty
$$

Step 2. With respect to $P_{n, \beta_{n}, K_{n}} \times Q, S_{n} / n^{1-\gamma}+W_{n} / n^{1 / 2-\gamma}$ satisfies the Laplace principle with exponential speed $n^{u}$ and rate function $\Gamma$.

According to Theorem 1.3.3 in [9], if we prove Step 1 and Step 2, then with respect to $P_{n, \beta_{n}, K_{n}}, S_{n} / n^{1-\gamma}$ satisfies the Laplace principle with speed $n^{u}$ and rate function $\Gamma$; i.e., for any bounded, continuous function $\psi$

$$
\lim _{n \rightarrow \infty} \frac{1}{n^{u}} \log \int_{\Lambda^{n}} \exp \left[n^{u} \psi\left(S_{n} / n^{1-\gamma}\right)\right] d P_{n, \beta_{n}, K_{n}}=\sup _{x \in \mathbb{R}}\{\psi(x)-\Gamma(x)\}
$$

Since the Laplace principle implies the MDP (Thm 1.2.3 in [9]), Theorem 6.2 follows.

Next, we prove Step 1 and Step 2.

Proof of Step 1. Since $\beta_{n}$ and $K_{n}$ are bounded and uniformly positive over $n$, the sequence $\sigma_{n}^{2}$ is bounded and uniformly positive over $n$. We have

$$
\begin{aligned}
Q\left\{\left|W_{n} / n^{1 / 2-\gamma}\right|>\delta\right\} & =Q\left\{\left|N\left(0, \sigma_{n}^{2}\right)\right|>n^{1 / 2-\gamma} \delta\right\} \\
& \leq \frac{\sqrt{2} \sigma_{n}}{\sqrt{\pi} n^{1 / 2-\gamma} \delta} \cdot \exp \left(-n^{1-2 \gamma} \delta^{2} /\left(2 \sigma_{n}^{2}\right)\right)
\end{aligned}
$$




$$
\frac{1}{n^{u}} \log Q\left\{\left|W_{n} / n^{1 / 2-\gamma}\right|>\delta\right\} \leq \frac{1}{n^{u}}\left[\log \frac{\sqrt{2} \sigma_{n}}{\sqrt{\pi} \delta}+\log \left(n^{\gamma-1 / 2}\right)-\frac{n^{1-2 \gamma} \delta^{2}}{2 \sigma_{n}^{2}}\right] .
$$

The limit of the right hand side of the last inequality is $-\infty$ since $u>0$ and $1-2 \gamma>u$. Thus (B.1) follows. The proof of Step 1 is done.

Proof of Step 2. Let $\psi$ be an arbitrary bounded, continuous function. Choosing $\varphi=$ $\exp \left[n^{u} \psi\right]$ and $\bar{\gamma}=\gamma$ in Lemma 8.1 yields

$$
\begin{aligned}
& \int_{\Lambda^{n} \times \Omega} \exp \left[n^{u} \psi\left(\frac{S_{n}}{n^{1-\gamma}}+\frac{W_{n}}{n^{1 / 2-\gamma}}\right)\right] d\left(P_{n, \beta_{n}, K_{n}} \times Q\right) \\
& =\frac{1}{\int_{\mathbb{R}} \exp \left[-n G_{\beta_{n}, K_{n}}\left(x / n^{\gamma}\right)\right] d x} \cdot \int_{\mathbb{R}} \exp \left[n^{u} \psi(x)-n G_{\beta_{n}, K_{n}}\left(x / n^{\gamma}\right)\right] d x .
\end{aligned}
$$

The proof of Step 2 rests on the following three properties of $n G_{\beta_{n}, K_{n}}\left(x / n^{\gamma}\right)$.

1. By hypothesis (iv) of Theorem 3.1 for $0<\alpha<\alpha_{0}$, there exists a polynomial $H$ satisfying $H(x) \rightarrow \infty$ as $|x| \rightarrow \infty$ together with the following property: $\exists R>0$ such that for $\forall n \in \mathbb{N}$ sufficiently large and $\forall x \in \mathbb{R}$ satisfying $\left|x / n^{\gamma}\right|<R$

$$
n G_{\beta_{n}, K_{n}}\left(x / n^{\gamma}\right) \geq n^{u} H(x)
$$

2. Let $B=\sup _{x \in \mathbb{R}}\{\psi(x)-g(x)\}$. Since $H(x) \rightarrow \infty$ and $g(x) \rightarrow \infty$ as $|x| \rightarrow \infty$, there exists $M>0$ with the following three properties:

$$
\sup _{|x|>M}\{\psi(x)-H(x)\} \leq-|B|-1
$$

the supremum of $\psi-g$ on $\mathbb{R}$ is attained on the interval $[-M, M]$, and the supremum of $-g$ on $\mathbb{R}$ is attained on the interval $[-M, M]$. In combination with item 1 , we have that for all $n \in \mathbb{N}$ satisfying $R n^{\gamma}>M$ 


$$
\begin{aligned}
& \sup _{\substack{M<|x|<R n^{\gamma}\\
}}\left\{n^{u} \psi(x)-n G_{\beta_{n}, K_{n}}\left(x / n^{\gamma}\right)\right\} \\
& \quad \leq \sup _{M<|x|<R n^{\gamma}}\left\{n^{u} \psi(x)-n^{u} H(x)\right\} \\
& \quad \leq-n^{u}(|B|+1) .
\end{aligned}
$$

3. Let $M$ be the number selected in item 2. By hypothesis (iii)(a) of Theorem 3.1 for $0<\alpha<\alpha_{0}$, for all $x \in \mathbb{R}$ satisfying $|x| \leq M, n^{1-u} G_{\beta_{n}, K_{n}}\left(x / n^{\gamma}\right)$ converges uniformly to $g(x)$ as $n \rightarrow \infty$.

Item 3 implies that for any $\delta>0$ and all sufficiently large $n$

$$
\begin{aligned}
& \exp \left(-n^{u} \delta\right) \int_{\{|x| \leq M\}} \exp \left[n^{u}(\psi(x)-g(x))\right] d x \\
& \quad \leq \int_{\{|x| \leq M\}} \exp \left[n^{u} \psi(x)-n G_{\beta_{n}, K_{n}}\left(x / n^{\gamma}\right)\right] d x \\
& \quad \leq \exp \left(n^{u} \delta\right) \int_{\{|x| \leq M\}} \exp \left[n^{u}(\psi(x)-g(x))\right] d x
\end{aligned}
$$

In addition, item 2 implies that

$$
\int_{\left\{M<|x|<R n^{\gamma}\right\}} \exp \left[n^{u} \psi(x)-n G_{\beta_{n}, K_{n}}\left(x / n^{\gamma}\right)\right] d x \leq 2 R n^{\gamma} \exp \left[-n^{u}(|B|+1)\right]
$$

Since $\psi$ is bounded, the last two displays show that there exists $a_{1}>0$ and $a_{2} \in \mathbb{R}$ such that for all sufficiently large $n$

$$
\int_{\left\{|x|<R n^{\gamma}\right\}} \exp \left[-n G_{\beta_{n}, K_{n}}\left(x / n^{\gamma}\right)\right] d x \leq a_{1} \exp \left(n^{u} a_{2}\right) .
$$

Since $u \in(0,1)$, by part (d) of Lemma 4.4 in [8] there exists $a_{3}>0$ such that for all sufficiently large $n$

$$
\int_{\left\{|x| \geq R n^{\gamma}\right\}} \exp \left[-n G_{\beta_{n}, K_{n}}\left(x / n^{\gamma}\right)\right] d x \leq 2 a_{1} \exp \left(-n a_{3}\right) .
$$


Together these three estimates show that for all sufficiently large $n$

$$
\begin{aligned}
\int_{\mathbb{R}} \exp & {\left[n^{u} \psi(x)-n G_{\beta_{n}, K_{n}}\left(x / n^{\gamma}\right)\right] d x } \\
= & \int_{\{|x| \leq M\}} \exp \left[n^{u} \psi(x)-n G_{\beta_{n}, K_{n}}\left(x / n^{\gamma}\right)\right] d x \\
& +\int_{\left\{M<|x|<R n^{\gamma}\right\}} \exp \left[n^{u} \psi(x)-n G_{\beta_{n}, K_{n}}\left(x / n^{\gamma}\right)\right] d x \\
& +\int_{\left\{|x| \geq R n^{\gamma}\right\}} \exp \left[n^{u} \psi(x)-n G_{\beta_{n}, K_{n}}\left(x / n^{\gamma}\right)\right] d x \\
\leq & \exp \left(n^{u} \delta\right) \int_{\{|x| \leq M\}} \exp \left[n^{u}(\psi(x)-g(x))\right] d x \\
& +2 R n^{\gamma} \exp \left[-n^{u}(|B|+1)\right]+2 a_{1} \exp \left(-n a_{3}+n^{u}\|\psi\|_{\infty}\right) .
\end{aligned}
$$

Hence for all sufficiently large $n$ we have

$$
\begin{aligned}
& \exp \left(-n^{u} \delta\right) \int_{\{|x| \leq M\}} \exp \left[n^{u}(\psi(x)-g(x))\right] d x \\
& \quad \leq \int_{\mathbb{R}} \exp \left[n^{u} \psi(x)-n G_{\beta_{n}, K_{n}}\left(x / n^{\gamma}\right)\right] d x \\
& \quad \leq \exp \left(n^{u} \delta\right) \int_{\{|x| \leq M\}} \exp \left[n^{u}(\psi(x)-g(x))\right] d x+\delta_{n},
\end{aligned}
$$

where

$$
\begin{aligned}
\delta_{n} & \leq 2 R n^{\gamma} \exp \left[-n^{u}(|B|+1)\right]+2 a_{1} \exp \left(-n a_{3}+n^{u}|| \psi \|_{\infty}\right) \\
& \leq 4 R n^{\gamma} \exp \left[-n^{u}(|B|+1)\right] .
\end{aligned}
$$

It follows that 


$$
\begin{aligned}
& \liminf _{n \rightarrow \infty} \frac{1}{n^{u}} \log \left[\exp \left(-n^{u} \delta\right) \int_{\{|x| \leq M\}} \exp \left[n^{u}(\psi(x)-g(x))\right] d x\right] \\
& \quad \leq \liminf _{n \rightarrow \infty} \frac{1}{n^{u}} \log \int_{\mathbb{R}} \exp \left[n^{u} \psi(x)-n G_{\beta_{n}, K_{n}}\left(x / n^{\gamma}\right)\right] d x \\
& \quad \leq \limsup _{n \rightarrow \infty} \frac{1}{n^{u}} \log \int_{\mathbb{R}} \exp \left[n^{u} \psi(x)-n G_{\beta_{n}, K_{n}}\left(x / n^{\gamma}\right)\right] d x \\
& \quad \leq \limsup _{n \rightarrow \infty} \frac{1}{n^{u}} \log \left[\exp \left(n^{u} \delta\right) \int_{\{|x| \leq M\}} \exp \left[n^{u}(\psi(x)-g(x))\right] d x+\delta_{n}\right] .
\end{aligned}
$$

By Laplace's method applied to the continuous function $\psi-g$ on $|x| \leq M$ and the fact that the supremum of $\psi-g$ on $\mathbb{R}$ is attained on the interval $[-M, M]$, we have

$$
\begin{aligned}
\lim _{n \rightarrow \infty} & \frac{1}{n^{u}} \log \int_{\{|x| \leq M\}} \exp \left[n^{u}(\psi(x)-g(x))\right] d x \\
= & \sup _{|x| \leq M}\{\psi(x)-g(x)\}=\sup _{x \in \mathbb{R}}\{\psi(x)-g(x)\} .
\end{aligned}
$$

Hence the first line of (B.5) equals

$$
\begin{aligned}
\liminf _{n \rightarrow \infty} & \frac{1}{n^{u}}\left[-n^{u} \delta+\log \int_{\{|x| \leq M\}} \exp \left[n^{u}(\psi(x)-g(x))\right] d x\right] \\
= & -\delta+\liminf _{n \rightarrow \infty} \frac{1}{n^{u}} \log \int_{\{|x| \leq M\}} \exp \left[n^{u}(\psi(x)-g(x))\right] d x \\
& =-\delta+\sup _{x \in \mathbb{R}}\{\psi(x)-g(x)\} .
\end{aligned}
$$

We have to work harder to evaluate the last line of (B.5). At the end of the proof we will show that the term $\delta_{n}$ can be neglected in evaluating the last line of (B.5); i.e.,

$$
\begin{aligned}
& \limsup _{n \rightarrow \infty} \frac{1}{n^{u}} \log \left[\exp \left(n^{u} \delta\right) \int_{\{|x| \leq M\}} \exp \left[n^{u}(\psi(x)-g(x))\right] d x+\delta_{n}\right] \\
& \quad=\limsup _{n \rightarrow \infty} \frac{1}{n^{u}} \log \left[\exp \left(n^{u} \delta\right) \int_{\{|x| \leq M\}} \exp \left[n^{u}(\psi(x)-g(x))\right] d x\right] .
\end{aligned}
$$

Under the assumption that this is true, by (B.6) the last line of (B.5) equals 


$$
\begin{aligned}
& \limsup _{n \rightarrow \infty} \frac{1}{n^{u}} \log \left[\exp \left(n^{u} \delta\right) \int_{\{|x| \leq M\}} \exp \left[n^{u}(\psi(x)-g(x))\right] d x\right] \\
& \quad=\limsup _{n \rightarrow \infty} \frac{1}{n^{u}}\left[n^{u} \delta+\log \int_{\{|x| \leq M\}} \exp \left[n^{u}(\psi(x)-g(x))\right] d x\right] \\
& \quad=\delta+\lim _{n \rightarrow \infty} \frac{1}{n^{u}} \log \int_{\{|x| \leq M\}} \exp \left[n^{u}(\psi(x)-g(x))\right] d x \\
& \quad=\delta+\sup _{x \in \mathbb{R}}\{\psi(x)-g(x)\} .
\end{aligned}
$$

Since $\delta>0$ is arbitrary, combining (B.5), (B.7), and (B.9) yields

$$
\lim _{n \rightarrow \infty} \frac{1}{n^{u}} \log \int_{\mathbb{R}} \exp \left[n^{u} \psi(x)-n G_{\beta_{n}, K_{n}}\left(x / n^{\gamma}\right)\right] d x=\sup _{x \in \mathbb{R}}\{\psi(x)-g(x)\} .
$$

Using the fact that the supremum of $g$ is attained on the interval $[-M, M]$ (see item 2 in the proof of Step 2), we apply the limit in the last display to $\psi=0$. We conclude from (B.2) that

$$
\begin{aligned}
\lim _{n \rightarrow \infty} & \frac{1}{n^{u}} \log \int_{\Lambda^{n} \times \Omega} \exp \left[n^{u} \psi\left(\frac{S_{n}}{n^{1-\gamma}}+\frac{W_{n}}{n^{1 / 2-\gamma}}\right)\right] d\left(P_{n, \beta_{n}, K_{n}} \times Q\right) \\
= & \lim _{n \rightarrow \infty} \frac{1}{n^{u}} \log \int_{\mathbb{R}} \exp \left[n^{u} \psi(x)-n G_{\beta_{n}, K_{n}}\left(x / n^{\gamma}\right)\right] d x \\
& -\lim _{n \rightarrow \infty} \frac{1}{n^{u}} \log \int_{\mathbb{R}} \exp \left[-n G_{\beta_{n}, K_{n}}\left(x / n^{\gamma}\right)\right] d x \\
= & \sup _{x \in \mathbb{R}}\{\psi(x)-g(x)\}+\sup _{x \in \mathbb{R}}\{-g(x)\} \\
= & \sup _{x \in \mathbb{R}}\{\psi(x)-g(x)\}+\inf _{y \in \mathbb{R}} g(y) \\
= & \sup _{x \in \mathbb{R}}\{\psi(x)-\Gamma(x)\} .
\end{aligned}
$$

Except for the proof of (B.8) we have completed the proof of Step 2, which show that with respect to $P_{n, \beta_{n}, K_{n}} \times Q, S_{n} / n^{1-\gamma}+W_{n} / n^{1 / 2-\gamma}$ satisfies the Laplace principle with exponential speed $n^{u}$ and rate function $\Gamma$.

To prove (B.8) we define

$$
A_{n}=\exp \left(n^{u} \delta\right) \int_{\{|x| \leq M\}} \exp \left[n^{u}(\psi(x)-g(x))\right] d x
$$


It suffices to show that $\delta_{n} / A_{n} \rightarrow 0$. To see this, we rewrite (B.8) as follows:

$$
\begin{aligned}
\lim & \sup \frac{1}{n^{u}} \log \left(A_{n}+\delta_{n}\right) \\
& =\limsup \frac{1}{n^{u}} \log \left[A_{n}\left(1+\frac{\delta_{n}}{A_{n}}\right)\right] \\
& =\limsup \left[\frac{1}{n^{u}} \log A_{n}+\frac{1}{n^{u}} \log \left(1+\frac{\delta_{n}}{A_{n}}\right)\right] \\
& =\limsup \frac{1}{n^{u}} \log A_{n} .
\end{aligned}
$$

Now we prove that $\delta_{n} / A_{n} \rightarrow 0$. By (B.6) we have

$$
\begin{aligned}
\lim \frac{1}{n^{u}} \log A_{n} & =\delta+\lim _{n \rightarrow \infty} \frac{1}{n^{u}} \log \int_{\{|x| \leq M\}} \exp \left[n^{u}(\psi(x)-g(x))\right] d x \\
& =\delta+\sup _{x \in \mathbb{R}}\{\psi(x)-g(x)\} \\
& =\delta+B
\end{aligned}
$$

which implies that for all sufficiently large $n$

$$
A_{n} \geq \exp \left[n^{u}\left(\frac{\delta}{2}+B\right)\right]
$$

Since by (B.4) we have for all sufficiently large $n$

$$
\delta_{n} \leq 4 R n^{\gamma} \exp \left[-n^{u}(|B|+1)\right]
$$

it follows that for any $0<\varepsilon<1$ and all sufficiently large $n$

$$
\delta_{n} \leq \exp \left[n^{u}(-|B|-1+\varepsilon)\right]
$$

and thus

$$
0 \leq \frac{\delta_{n}}{A_{n}} \leq \exp \left[n^{u}\left(-|B|-1+\varepsilon-\frac{\delta}{2}-B\right)\right]
$$


If $B \geq 0$, then

$$
-|B|-1+\varepsilon-\frac{\delta}{2}-B=-1+\varepsilon-\frac{\delta}{2}-2 B<0 .
$$

If $B<0$, then

$$
-|B|-1+\varepsilon-\frac{\delta}{2}-B=-1+\varepsilon-\frac{\delta}{2}<0 .
$$

Thus in all cases the limit of the right hand side of (B.11) is 0 . This completes the proof of (B.8).

Together Step 1 and Step 2 prove that with respect to $P_{n, \beta_{n}, K_{n}}, S_{n} / n^{1-\gamma}$ satisfies the Laplace principle with speed $n^{u}$ and rate function $\Gamma$. The proof of Theorem 6.2 is complete. 


\section{APPENDIX C \\ PROOF OF THE REPRESENTATION FORMULA IN LEMMA 8.1 FOR THE MEAN-FIELD B-C MODEL}

In this appendix we prove the representation formula in Lemma 8.1. For easy reference this lemma is restated in Lemma C.1.

Lemma C.1. Given a positive sequence $\left(\beta_{n}, K_{n}\right)$, let $W_{n}$ be a sequence of normal random variables with mean 0 and variance $\left(2 \beta_{n} K_{n}\right)^{-1}$ defined on a probability space $(\Omega, \mathcal{F}, Q)$. Then for any $\bar{\gamma} \in[0,1)$ and any bounded, measurable function $\varphi$

$$
\begin{aligned}
& \int_{\Lambda^{n} \times \Omega} \varphi\left(S_{n} / n^{1-\bar{\gamma}}+W_{n} / n^{1 / 2-\bar{\gamma}}\right) d\left(P_{n, \beta_{n}, K_{n}} \times Q\right) \\
& \quad=\frac{1}{\int_{\mathbb{R}} \exp \left[-n G_{\beta_{n}, K_{n}}\left(x / n^{\bar{\gamma}}\right)\right] d x} \cdot \int_{\mathbb{R}} \varphi(x) \exp \left[-n G_{\beta_{n}, K_{n}}\left(x / n^{\bar{\gamma}}\right)\right] d x .
\end{aligned}
$$

In this formula $G_{\beta_{n}, K_{n}}$ is the free energy function defined in (2.4).

We offer two proofs of the representation formula (C.1). An interesting feature of this formula is that the addition of the term $W_{n} / n^{1 / 2-\bar{\gamma}}$ allows one to express the $P_{n, \beta_{n}, K_{n}} \times Q$ distribution of the argument of $\varphi$ on the left-hand side of (C.1) in the simple form given on the right-hand side of (C.1). As we show after proof 2 of the representation formula, the technique used in this proof explains why the additional term $W_{n} / n^{1 / 2-\bar{\gamma}}$ is the correct choice. In this sense proof 2 motivates the representation formula nicely.

Proof 1 of the representation formula is based on expressing the distribution function of $S_{n}+\sqrt{n} W_{n}$ in terms of a convolution. This proof follows the pattern of the proof of Lemma 3.3 in [12], which applies to the Curie-Weiss model, and Lemma 3.2 in [15], which 
applies to the Curie-Weiss-Potts model. Proof 2 of the representation formula is based on the Gaussian transform (C.7), which allows us to rewrite the term $\exp \left[\beta K\left(S_{n}\right)^{2} / n\right]$ in the Hamiltonian. This procedure is known in the physics literature as the HubbardStratonovich transformation.

Proof 1. In order to simplify the notation, we write $\beta, K$, and $W$ in place of $\beta_{n}, K_{n}$, and $W_{n}$. Let $F_{n}$ denote the distribution function of $P_{n, \beta, K}\left(S_{n} \in d y\right), \Phi_{n}$ the distribution function of $\sqrt{n} W$, and $f_{n}$ the density function of $Q(\sqrt{n} W \in d x)$, where $W$ is a normal random variable with mean 0 and variance $(2 \beta K)^{-1}$ defined on a probability space $(\Omega, \mathcal{F}, Q)$. We claim that

$$
P_{n, \beta, K} \times Q\left(S_{n}+\sqrt{n} W \in d u\right)=\left[\int_{\mathbb{R}} f_{n}(u-y) F_{n}(d y)\right] d u
$$

i.e., the density function of the measure $P_{n, \beta, K}\left(S_{n}+\sqrt{n} W \in d u\right)$ is the function multiplying $d u$ on the right-hand side of (C.2).

In order to prove this claim, we use the independence of $S_{n}$ and $W$, equation (31) in $\S I I .8$ on page 241 of [17], and Fubini's Theorem to write for any $z \in \mathbb{R}$

$$
\begin{aligned}
P_{n, \beta, K} \times Q\left(S_{n}+\sqrt{n} W \leq z\right) & =\int_{\mathbb{R}} \Phi_{n}(z-y) F_{n}(d y) \\
& =\int_{\mathbb{R}}\left[\int_{-\infty}^{z-y} f_{n}(u) d u\right] F_{n}(d y) \\
& =\int_{\mathbb{R}}\left[\int_{-\infty}^{z} f_{n}(u-y) d u\right] F_{n}(d y) \\
& =\int_{-\infty}^{z}\left[\int_{\mathbb{R}} f_{n}(u-y) F_{n}(d y)\right] d u \\
& =\int_{-\infty}^{z}\left[\int_{\mathbb{R}} f_{n}(u-y) P_{n, \beta, K}\left(S_{n} \in d y\right)\right] d u
\end{aligned}
$$

Since the distribution function $P_{n, \beta, K} \times Q\left(S_{n}+\sqrt{n} W \leq z\right)$ uniquely determines the measure $P_{n, \beta, K} \times Q\left(S_{n}+\sqrt{n} W \in d u\right)$, the claim (C.2) is proved. 
By claim (C.2) we write the left-hand side of (C.1) as

$$
\begin{aligned}
\int_{\Lambda^{n} \times \Omega} \varphi\left(S_{n} / n^{1-\bar{\gamma}}+W / n^{1 / 2-\bar{\gamma}}\right) d\left(P_{n, \beta, K} \times Q\right) \\
=\int_{\mathbb{R}} \varphi\left(u / n^{1-\bar{\gamma}}\right) P_{n, \beta, K} \times Q\left(S_{n}+\sqrt{n} W \in d u\right) \\
=\int_{\mathbb{R}} \varphi\left(u / n^{1-\bar{\gamma}}\right)\left[\int_{\mathbb{R}} f_{n}(u-y) F_{n}(d y)\right] d u \\
=\int_{\mathbb{R}} \varphi\left(u / n^{1-\bar{\gamma}}\right)\left[\int_{\mathbb{R}} f_{n}(u-y) P_{n, \beta, K}\left(S_{n} \in d y\right)\right] d u
\end{aligned}
$$

The random variable $\sqrt{n} W$ has the density function

$$
f_{n}(x)=\left(\frac{\beta K}{n \pi}\right)^{1 / 2} \exp \left[-\frac{\beta K}{n} x^{2}\right]
$$

and as shown in (2.2),

$$
P_{n, \beta, K}\left(S_{n} \in d y\right)=\frac{1}{\tilde{Z}_{n}(\beta, K)} \cdot \exp \left[\frac{\beta K}{n} y^{2}\right] P_{n, \beta}\left(S_{n} \in d y\right)
$$

We write

$$
A_{n}=\left(\frac{\beta K}{n \pi}\right)^{1 / 2} \cdot \frac{1}{\tilde{Z}_{n}(\beta, K)} .
$$

Since for $\omega \in \Lambda^{n}, P_{n, \beta}(d \omega)=\prod_{j=1}^{n} \rho_{\beta}\left(d \omega_{j}\right)$, it follows from (C.3) that 


$$
\begin{aligned}
\int_{\Lambda^{n} \times \Omega} \varphi\left(S_{n} / n^{1-\bar{\gamma}}+W / n^{1 / 2-\bar{\gamma}}\right) d\left(P_{n, \beta, K} \times Q\right) \\
=\int_{\mathbb{R}} \varphi\left(u / n^{1-\bar{\gamma}}\right) \\
\quad \times\left[\int_{\mathbb{R}}\left(\frac{\beta K}{n \pi}\right)^{1 / 2} \exp \left[-\frac{\beta K}{n}(u-y)^{2}\right] \cdot \frac{1}{\tilde{Z}_{n}(\beta, K)} \cdot \exp \left[\frac{\beta K}{n} y^{2}\right] P_{n, \beta}\left(S_{n} \in d y\right)\right] d u \\
=A_{n} \cdot \int_{\mathbb{R}} \varphi\left(u / n^{1-\bar{\gamma}}\right)\left[\int_{\mathbb{R}} \exp \left(-\frac{\beta K}{n}\left[(u-y)^{2}-y^{2}\right]\right) P_{n, \beta}\left(S_{n} \in d y\right)\right] d u \\
=A_{n} \cdot \int_{\mathbb{R}} \varphi\left(u / n^{1-\bar{\gamma}}\right) \exp \left(-\frac{\beta K}{n} u^{2}\right) \times\left[\int_{\mathbb{R}} \exp \left(\frac{2 \beta K}{n} u y\right) P_{n, \beta}\left(S_{n} \in d y\right)\right] d u \\
=A_{n} \cdot \int_{\mathbb{R}} \varphi\left(u / n^{1-\bar{\gamma}}\right) \exp \left(-\frac{\beta K}{n} u^{2}\right) \times\left[\int_{\Lambda^{n}} \exp \left(\frac{2 \beta K}{n} u S_{n}\right) d P_{n, \beta}\right] d u \\
=A_{n} \cdot \int_{\mathbb{R}} \varphi\left(u / n^{1-\bar{\gamma}}\right) \exp \left(-\frac{\beta K}{n} u^{2}\right) \times\left[\int_{\Lambda} \exp \left(\frac{2 \beta K}{n} u \omega_{1}\right) \rho_{\beta}\left(d \omega_{1}\right)\right] d u \\
=A_{n} \cdot \int_{\mathbb{R}} \varphi\left(u / n^{1-\bar{\gamma}}\right) \exp \left(-\left[\frac{\beta K}{n} u^{2}-n c_{\beta}\left(\frac{2 \beta K}{n} u\right)\right]\right) d u \\
=A_{n} \cdot \int_{\mathbb{R}} \varphi\left(u / n^{1-\bar{\gamma}}\right) \exp \left[-n G_{\beta, K}(u / n)\right] d u \\
=A_{n} \cdot n^{1-\bar{\gamma}} \varphi \int_{\mathbb{R}} \varphi(x) \exp \left[-n G_{\beta, K}\left(x / n^{\bar{\gamma}}\right)\right] d x
\end{aligned}
$$

Setting $\varphi \equiv 1$, we see that

$$
A_{n} \cdot n^{1-\bar{\gamma}}=\frac{1}{\int_{\mathbb{R}} \exp \left[-n G_{\beta, K}\left(x / n^{\bar{\gamma}}\right)\right] d x} .
$$

We conclude that

$$
\begin{aligned}
& \int_{\Lambda^{n} \times \Omega} \varphi\left(S_{n} / n^{1-\bar{\gamma}}+W_{n} / n^{1 / 2-\bar{\gamma}}\right) d\left(P_{n, \beta_{n}, K_{n}} \times Q\right) \\
& \quad=\frac{1}{\int_{\mathbb{R}} \exp \left[-n G_{\beta_{n}, K_{n}}\left(x / n^{\bar{\gamma}}\right)\right] d x} \cdot \int_{\mathbb{R}} \varphi(x) \exp \left[-n G_{\beta_{n}, K_{n}}\left(x / n^{\bar{\gamma}}\right)\right] d x .
\end{aligned}
$$

This completes proof 1 of the lemma.

Proof 2. As in proof 1, we write $\beta, K$, and $W$ in place of $\beta_{n}, K_{n}$, and $W_{n}$. Formula (2.2) gives 


$$
P_{n, \beta, K}(d \omega)=\frac{1}{\tilde{Z}_{n}(\beta, K)} \exp \left[\frac{\beta K}{n}\left(S_{n}\right)^{2}\right] P_{n, \beta}(d \omega) .
$$

where $P_{n, \beta}(d \omega)=\prod_{j=1}^{n} \rho_{\beta}\left(d \omega_{j}\right)$ and $\tilde{Z}_{n}(\beta, K)=\int_{\Lambda^{n}} \exp \left[\frac{\beta K}{n}\left(S_{n}\right)^{2}\right] d P_{n, \beta}$.

As shown on page 408 of [2], Lemma C.1 follows if we can prove (C.1) for $\varphi(t)=e^{\lambda t}$ for all $\lambda \in \mathbb{R}$; in symbols,

$$
\begin{aligned}
& \int_{\Lambda^{n} \times \Omega} \exp \left[\frac{\lambda S_{n}}{n^{1-\bar{\gamma}}}+\frac{\lambda W}{n^{1 / 2-\bar{\gamma}}}\right] d\left(P_{n, \beta, K} \times Q\right) \\
& =\frac{1}{\int_{\mathbb{R}} \exp \left[-n G_{\beta, K}\left(x / n^{\bar{\gamma}}\right)\right] d x} \cdot \int_{\mathbb{R}} e^{\lambda x} \cdot \exp \left[-n G_{\beta, K}\left(x / n^{\bar{\gamma}}\right)\right] d x .
\end{aligned}
$$

Given a random variable $X$, we denote its moment generating function as $M(X, \lambda)$ for $\lambda \in \mathbb{R}$. We start the proof by writing the left-hand side of (C.5) in the form

$$
\begin{aligned}
\int_{\Lambda^{n} \times \Omega} & \exp \left[\frac{\lambda S_{n}}{n^{1-\bar{\gamma}}}+\frac{\lambda W}{n^{1 / 2-\bar{\gamma}}}\right] d\left(P_{n, \beta, K} \times Q\right) \\
= & \left(\int_{\Lambda^{n}} \exp \left[\frac{\lambda S_{n}}{n^{1-\bar{\gamma}}}\right] d P_{n, \beta, K}\right) \times\left(\int_{\Omega} \exp \left[\frac{\lambda W}{n^{1 / 2-\bar{\gamma}}}\right] d Q\right) \\
= & M\left(S_{n} / n^{1-\bar{\gamma}}, \lambda\right) \times M\left(W / n^{1 / 2-\bar{\gamma}}, \lambda\right) .
\end{aligned}
$$

Since $\sqrt{n} W$ has the density function

$$
\left(\frac{\beta K}{n \pi}\right)^{1 / 2} \exp \left[-\frac{\beta K}{n} x^{2}\right]
$$

we have

$$
M\left(W / n^{1 / 2-\bar{\gamma}}, \lambda\right)=\int_{\mathbb{R}} \exp \left[\frac{\lambda}{n^{1-\bar{\gamma}}} x\right] \cdot\left(\frac{\beta K}{n \pi}\right)^{1 / 2} \exp \left[-\frac{\beta K}{n} x^{2}\right] d x
$$

The Gaussian transform

$$
e^{\frac{1}{2} \alpha^{2}}=\frac{1}{\sqrt{2 \pi}} \int_{\mathbb{R}} e^{-v^{2} / 2} \cdot e^{\alpha v} d v
$$


with $\alpha=(2 \beta K / n)^{1 / 2} S_{n}$ allows us to write in (C.4)

$$
\exp \left[\frac{\beta K}{n}\left(S_{n}\right)^{2}\right]=\exp \left[\frac{1}{2} \alpha^{2}\right]=\frac{1}{\sqrt{2 \pi}} \int_{\mathbb{R}} e^{-v^{2} / 2} \cdot \exp \left(\left(\frac{2 \beta K}{n}\right)^{1 / 2} S_{n} v\right) d v
$$

We use (C.4) and (C.8) to write

$$
\begin{aligned}
M\left(S_{n} / n^{1-\bar{\gamma}}, \lambda\right)= & \int_{\Lambda^{n}} \exp \left[\frac{\lambda}{n^{1-\bar{\gamma}}} S_{n}\right] \cdot \frac{1}{\tilde{Z}_{n}(\beta, K)} \exp \left[\frac{\beta K}{n}\left(S_{n}\right)^{2}\right] P_{n, \beta} \\
= & \frac{1}{\sqrt{2 \pi}} \cdot \frac{1}{\tilde{Z}_{n}(\beta, K)} \int_{\Lambda^{n}} \exp \left[\frac{\lambda}{n^{1-\bar{\gamma}}} S_{n}\right] \\
& \times\left[\int_{\mathbb{R}} e^{-v^{2} / 2} \cdot \exp \left(\left(\frac{2 \beta K}{n}\right)^{1 / 2} S_{n} v\right) d v\right] d P_{n, \beta} \\
= & \frac{1}{\sqrt{2 \pi}} \cdot \frac{1}{\tilde{Z}_{n}(\beta, K)} \\
& \times \int_{\mathbb{R}} e^{-v^{2} / 2}\left[\int_{\Lambda^{n}} \exp \left[\left(\frac{\lambda}{n^{1-\bar{\gamma}}}+\left(\frac{2 \beta K}{n}\right)^{1 / 2} v\right) S_{n}\right] d P_{n, \beta}\right] d v .
\end{aligned}
$$

In order to simplify the calculation, we introduce a new variable $u$ by the formula

$$
u=\frac{v}{(2 \beta K / n)^{1 / 2}}+\frac{\left(\lambda / n^{1-\bar{\gamma}}\right)}{(2 \beta K / n)}
$$

Since

$$
\frac{\lambda}{n^{1-\bar{\gamma}}}+\left(\frac{2 \beta K}{n}\right)^{1 / 2} v=\frac{2 \beta K}{n} u
$$

and

$$
\frac{v^{2}}{2}=\left(\frac{\beta K}{n}\right) u^{2}-\frac{\lambda}{n^{1-\bar{\gamma}}} u+\frac{\left(\lambda / n^{1-\bar{\gamma}}\right)^{2}}{2 \cdot(2 \beta K / n)}
$$

it follows from (C.9) that

$$
\begin{aligned}
& M\left(S_{n} / n^{1-\bar{\gamma}}, \lambda\right) \\
& =\frac{1}{\sqrt{2 \pi}} \cdot \frac{1}{\tilde{Z}_{n}(\beta, K)} \int_{\mathbb{R}} \exp \left[-\frac{\beta K}{n} u^{2}+\frac{\lambda}{n^{1-\bar{\gamma}}} u-\frac{\left(\lambda / n^{1-\bar{\gamma}}\right)^{2}}{2 \cdot(2 \beta K / n)}\right] \\
& \quad \times\left[\int_{\Lambda^{n}} \exp \left(\frac{2 \beta K}{n} u S_{n}\right) d P_{n, \beta}\right] \cdot\left(\frac{2 \beta K}{n}\right)^{1 / 2} d u .
\end{aligned}
$$


Multiplying the last expression by (C.6) and rearranging the order of terms, we obtain

$$
\begin{aligned}
& M\left(S_{n} / n^{1-\bar{\gamma}}, \lambda\right) \times M\left(W / n^{1 / 2-\bar{\gamma}}, \lambda\right) \\
&=\left(\frac{\beta K}{n \pi}\right)^{1 / 2} \cdot \frac{1}{\tilde{Z}_{n}(\beta, K)} \cdot \frac{1}{\sqrt{2 \pi}} \cdot\left(\frac{2 \beta K}{n}\right)^{1 / 2} \cdot \exp \left[-\frac{\left(\lambda / n^{1-\bar{\gamma}}\right)^{2}}{2(2 \beta K / n)}\right] \\
& \times \int_{\mathbb{R}} \exp \left[\frac{\lambda}{n^{1-\bar{\gamma}}} x\right] \cdot \exp \left[-\frac{\beta K}{n} x^{2}\right] d x \\
& \times \int_{\mathbb{R}} \exp \left[\frac{\lambda}{n^{1-\bar{\gamma}}} u\right] \cdot \exp \left[-\frac{\beta K}{n} u^{2}\right] \cdot\left[\int_{\Lambda^{n}} \exp \left(\frac{2 \beta K}{n} u S_{n}\right) d P_{n, \beta}\right] d u
\end{aligned}
$$

The last three factors in the second line of (C.11) and the integral in the third line of (C.11) can be combined into the integral of a normal density function, which is 1; i.e.,

$$
\begin{aligned}
& \frac{1}{\sqrt{2 \pi}} \cdot\left(\frac{2 \beta K}{n}\right)^{1 / 2} \cdot \exp \left[-\frac{\left(\lambda / n^{1-\bar{\gamma}}\right)^{2}}{2(2 \beta K / n)}\right] \times \int_{\mathbb{R}} \exp \left[\frac{\lambda}{n^{1-\bar{\gamma}}} x\right] \cdot \exp \left[-\frac{\beta K}{n} x^{2}\right] d x \\
& =\frac{1}{\sqrt{2 \pi}} \int_{\mathbb{R}} \exp \left[-\left(\frac{\beta K}{n} x^{2}-\frac{\lambda}{n^{1-\bar{\gamma}}} x+\frac{\left(\lambda / n^{1-\bar{\gamma}}\right)^{2}}{2(2 \beta K / n)}\right)\right]\left(\frac{2 \beta K}{n}\right)^{1 / 2} d x \\
& =\frac{1}{\sqrt{2 \pi}} \int_{\mathbb{R}} \exp \left[-\frac{\left(\left(\frac{2 \beta K}{n}\right)^{1 / 2} x-\frac{\lambda-n^{1-\bar{\gamma}}}{(2 \beta K / n)^{1 / 2}}\right)^{2}}{2}\right] d\left((2 \beta K / n)^{1 / 2} x\right) \\
& =1 .
\end{aligned}
$$

Define

$$
A_{n}=\left(\frac{\beta K}{n \pi}\right)^{1 / 2} \cdot \frac{1}{\tilde{Z}_{n}(\beta K)} .
$$

Since for $\omega \in \Lambda^{n} P_{n, \beta}(d \omega)=\prod_{j=1}^{n} \rho_{\beta}\left(d \omega_{j}\right)$, it follows from (C.11) that 


$$
\begin{aligned}
M & \left(S_{n} / n^{1-\bar{\gamma}}, \lambda\right) \times M\left(W / n^{1 / 2-\bar{\gamma}}, \lambda\right) \\
& =A_{n} \cdot \int_{\mathbb{R}} \exp \left[\frac{\lambda}{n^{1-\bar{\gamma}}} u\right] \cdot \exp \left[-\frac{\beta K}{n} u^{2}\right] \cdot\left[\int_{\Lambda^{n}} \exp \left(\frac{2 \beta K}{n} u S_{n}\right) d P_{n, \beta}\right] d u \\
& =A_{n} \cdot \int_{\mathbb{R}} \exp \left[\frac{\lambda}{n^{1-\bar{\gamma}}} u\right] \cdot \exp \left[-\frac{\beta K}{n} u^{2}\right] \cdot\left[\int_{\Lambda} \exp \left(\frac{2 \beta K}{n} u \omega_{1}\right) \rho_{\beta}\left(d \omega_{1}\right)\right]^{n} d u \\
& =A_{n} \cdot \int_{\mathbb{R}} e^{\lambda\left(u / n^{1-\bar{\gamma}}\right)} \cdot \exp \left(-\left[\frac{\beta K}{n} u^{2}-n c_{\beta}\left(\frac{2 \beta K}{n} u\right)\right]\right) d u \\
& =A_{n} \cdot \int_{\mathbb{R}} e^{\lambda\left(u / n^{1-\bar{\gamma}}\right)} \cdot \exp \left[-n G_{\beta, K}(u / n)\right] d u \\
& =A_{n} \cdot n^{1-\bar{\gamma}} \int_{\mathbb{R}} e^{\lambda x} \cdot \exp \left[-n G_{\beta, K}\left(x / n^{\bar{\gamma}}\right)\right] d x
\end{aligned}
$$

where the definitions of the cumulant generation function $c_{\beta}$ and the free-energy function $G_{\beta, K}$ are given in (2.3) and (2.4). Setting $\lambda \equiv 0$, we see that

$$
A_{n} \cdot n^{1-\bar{\gamma}}=\frac{1}{\int_{\mathbb{R}} \exp \left[-n G_{\beta, K}\left(x / n^{\bar{\gamma}}\right)\right] d x} .
$$

We conclude that

$$
\begin{aligned}
& \int_{\Lambda^{n} \times \Omega} \exp \left[\frac{\lambda S_{n}}{n^{1-\bar{\gamma}}}+\frac{\lambda W}{n^{1 / 2-\bar{\gamma}}}\right] d\left(P_{n, \beta, K} \times Q\right) \\
& \quad=M\left(S_{n} / n^{1-\bar{\gamma}}, \lambda\right) \times M\left(S_{n} / n^{1-\bar{\gamma}}, \lambda\right) \\
& \quad=\frac{1}{\int_{\mathbb{R}} \exp \left[-n G_{\beta, K}\left(x / n^{\bar{\gamma}}\right)\right] d x} \cdot \int_{\mathbb{R}} e^{\lambda x} \cdot \exp \left[-n G_{\beta, K}\left(x / n^{\bar{\gamma}}\right)\right] d x
\end{aligned}
$$

which implies (C.1) holds for any bounded, measurable function $\varphi$. This completes proof 2 of the lemma.

We now show how the technique used in proof 2 explains the appearance of the random variables $W_{n} / n^{1 / 2-\bar{\gamma}}$ in the representation formula (C.1). The problem is to find random variables $X_{n}$ such that for any $\bar{\gamma} \in[0,1)$ and any bounded, measurable function $\varphi$

$$
\begin{aligned}
& \int_{\Lambda^{n} \times \Omega} \varphi\left(S_{n} / n^{1-\bar{\gamma}}+X_{n}\right) d\left(P_{n, \beta_{n}, K_{n}} \times Q\right) \\
& \quad=\frac{1}{\int_{\mathbb{R}} \exp \left[-n G_{\beta_{n}, K_{n}}\left(x / n^{\bar{\gamma}}\right)\right] d x} \cdot \int_{\mathbb{R}} \varphi(x) \exp \left[-n G_{\beta_{n}, K_{n}}\left(x / n^{\bar{\gamma}}\right)\right] d x
\end{aligned}
$$


It suffices to prove this with $\varphi(t)$ replaced by $e^{\lambda t}$ for any $\lambda \in \mathbb{R}$. Doing this, we obtain the following formula for the moment generating function of $X_{n}$ :

$$
\begin{aligned}
M\left(X_{n}, \lambda\right) & =\frac{1}{\int_{\mathbb{R}} \exp \left[-n G_{\beta_{n}, K_{n}}\left(x / n^{\bar{\gamma}}\right)\right] d x} \cdot \frac{\int_{\mathbb{R}} e^{\lambda x} \cdot \exp \left[-n G_{\beta_{n}, K_{n}}\left(x / n^{\bar{\gamma}}\right)\right] d x}{M\left(S_{n} / n^{1-\bar{\gamma}}, \lambda\right)} \\
& =\frac{1}{n^{1-\bar{\gamma}} \cdot \int_{\mathbb{R}} \exp \left[-n G_{\beta_{n}, K_{n}}\left(x / n^{\bar{\gamma}}\right)\right] d x} \cdot \frac{n^{1-\bar{\gamma}} \cdot \int_{\mathbb{R}} e^{\lambda x} \cdot \exp \left[-n G_{\beta_{n}, K_{n}}\left(x / n^{\bar{\gamma}}\right)\right] d x}{M\left(S_{n} / n^{1-\bar{\gamma}}, \lambda\right)} .
\end{aligned}
$$

If we do proof 2 in reverse, then (C.13) reduces to

$$
M\left(X_{n}, \lambda\right)=\int_{\mathbb{R}} \exp \left[\frac{\lambda}{n^{1-\bar{\gamma}}} x\right] \cdot\left(\frac{\beta K}{n \pi}\right)^{1 / 2} \exp \left[-\frac{\beta K}{n} x^{2}\right] d x
$$

This shows that $n^{1-\bar{\gamma}} X_{n}$ has the density function

$$
\left(\frac{\beta K}{n \pi}\right)^{1 / 2} \exp \left[-\frac{\beta K}{n} x^{2}\right]
$$

which is the density function of $\sqrt{n} W_{n}$. Hence the random variables $X_{n}$ we found is $W_{n} / n^{1 / 2-\bar{\gamma}}$ 


\section{BIBLIOGRAPHY}

[1] M. N. Barber, Finite-size Scaling, Phase Transitions and Critical Phenomena, Vol 8, 145-266, C. Domb and J. Lebowitz, editors, Academic Press, London, 1983.

[2] P. Billingsley, Probability and Measure, Second Edition, John Wiley \& Sons, New York, 1986.

[3] M. Blume, Theory of the first-order magnetic phase change in $\mathrm{UO}_{2}$, Phys. Rev. 141 (1966) 517-524.

[4] M. Blume, V. J. Emery, and R. B. Griffiths. Ising model for the $\lambda$ transition and phase separation in $\mathrm{He}^{3}-\mathrm{He}^{4}$ mixtures. Phys. Rev. A 4:1071-1077, 1971.

[5] H. W. Capel. On the possibility of first-order phase transitions in Ising systems of triplet ions with zero-field splitting. Physica 32: 966-988, 1966.

[6] H. W. Capel. On the possibility of first-order phase transitions in Ising systems of triplet ions with zero-field splitting II. Physica 33:295-331, 1967.

[7] H. W. Capel. On the possibility of first-order phase transitions in Ising systems of triplet ions with zero-field splitting III. Physica 37:423-441, 1967.

[8] M. Costeniuc, R. S. Ellis, and P. T. Otto. Multiple critical behavior of probabilistic limit theorems in the neighborhood of a tricritical point. J. Stat. Phys. 127 (2007) 495-552.

[9] P. Dupuis and R. S. Ellis, A Weak Convergence Approach to the Theory of Large Deviations, John Wiley \& Sons, New York, 1997.

[10] R. S. Ellis, J. Machta, and P. T. Otto. Asymptotic behavior of the magnetization near critical and tricritical points via Ginzburg-Landau polynomials. J. Stat. Phys. 133 (2008) 101-129.

[11] R. S. Ellis, J. Machta, and P. T. Otto. Ginzburg-Landau polynomials and the asymptotic behavior of the magnetization near critical and tricritical points. Unpublished. http://arxiv.org/abs/0803.0178

[12] R. S. Ellis and C. M. Newman, Limit theorems for sums of dependent random variables occurring in statistical mechanics, Z. Wahrsch. verw. Geb. 44 (1979) 117-139.

[13] R. S. Ellis, J. Machta, and P. T. Otto. Asymptotic behavior of the finite-size magnetization as a function of the speed of approach to criticality, Ann. Appl. Prob. 20 (2010) $2118-2161$. 
[14] R. S. Ellis, P. T. Otto, and H. Touchette, Analysis of phase transitions in the mean-field Blume-Emery-Griffiths model, Ann. Appl. Prob. 15 (2005) 2203-2254.

[15] R. S. Ellis and K. Wang, Limit theorems for the empirical vector of the Curie-WeissPotts model, Stoch. Proc. Appl. 35 (1990) 59-79.

[16] W. Rudin, Real and Complex Analysis, Third Edition, McGraw-Hill, New York, 1987.

[17] A. N. Shiryaev, Probability, Second edition, translated by R. P. Boas, Springer, New York, 1995. 